\title{
Modulation of allergic airway inflammation by glucocorticoids
}

\author{
Dissertation \\ for the award of the degree \\ "Doctor rerum naturalium" \\ of the Georg-August-Universität Göttingen
}

within the doctoral program "Molecular Biology of Cells" of the Georg-August University School of Science (GAUSS)

submitted by

Anna Karabinskaya

from

Saint Petersburg, Russia

Göttingen, 2013 


\section{Thesis Committee}

\section{Prof. Dr. Holger Reichardt (First Referee)}

Dept. of Cellular and Molecular Immunology

University of Göttingen Medical School

\section{Prof. Dr. Uwe Groß (Second Referee)}

Dept. of Medical Microbiology

Institute for Medical Microbiology

University of Göttingen Medical School

\section{Prof. Dr. Jan Tuckermann}

Institute for General Zoology and Endocrinology

University of Ulm

\section{Additional members of the Examination Board}

\section{Prof. Dr. Heidi Hahn}

Institute of Human Genetics

University of Göttingen Medical School

\section{Prof. Dr. Frauke Alves}

Department of Hematology and Oncology

University of Göttingen Medical School

\section{Prof. Dr. Hubertus Jarry}

Department of Clinical and Experimental Endocrinology

University of Göttingen Medical School

\section{Prof. Dr. Lutz Walter}

Department of Primate Genetics

German Primate Center, Göttingen 


\section{Declaration}

The work presented in this thesis represents original work carried out by the author and has not been submitted in any form to any other university. It was written independently and with no other sources and aids than quoted.

August 2013

Göttingen, Germany

Anna Karabinskaya 


\begin{abstract}
A crucial step in the development of improved drugs for asthma therapy is a thorough understanding of the mechanisms, which are important for effective treatment, and the identification of target cells in asthma pathogenesis. In this work these two key questions were tackled using a murine model of asthma.
\end{abstract}

The analysis of the GC action mode revealed that the suppression of inflammation was strongly depended on DNA-binding and dimerisation of the GR. In absence of this molecular mechanism GC should no longer exert their anti-inflammatory functions in asthmatic mice despite the presence of the DNA-binding independent mode of GR action and non-genomic effects.

Analysis of the efficiency of GC therapy in different cell type-specific GR-deficient mice surprisingly demonstrated that treatability of AAI was independent on GR expression in immune cells. This notion was corroborated by experiments with bone marrow chimeras of $\mathrm{GR}^{\mathrm{dim}}$ mice that revealed that the efficiency of GC therapy was mostly dependent on effects on non-immune cells of the lungs. Additionally, an inducible GR knockout in AT2 epithelial cells led to a strongly diminished effectiveness of GCs in the suppression of the inflammatory reaction. This observation correlates with the importance of these cells in the regulation of innate and adaptive immune responses and the initiation of asthmatic reactions.

The gene expression analysis of $\mathrm{GR}^{\mathrm{dim}}$ mice, $\mathrm{GR}^{\mathrm{dim}}$ bone marrow chimeras and GR $\mathrm{GPCreERT2}^{\mathrm{S}}$ mice showed that the treatment with GCs exerts its anti-inflammatory functions via downregulation of mRNA expression in lung cells of the following genes: IL-4 and IL-13, IL-1 $\beta$ and IL-33, IL-25 and RANTES, TSLP and TNF- $\alpha$, Eotaxins (1\&2) and iNOS. The expression of IL-9 and $\beta$-Tryptase could also be important for asthma treatment because of the activation of mast cells, but based on the efficient treatability of mast cell deficient mice, these genes do not seem to be crucial for GC therapy. The treatment reduced mRNA expression of genes critical for the DC activation and could improve the barrier function via upregulation of ZO-1 mRNA expression. These effects of GC on gene expression of several inflammatory mediators demonstrate how important the regulation of epithelial cell activation is during asthmatic reaction. 


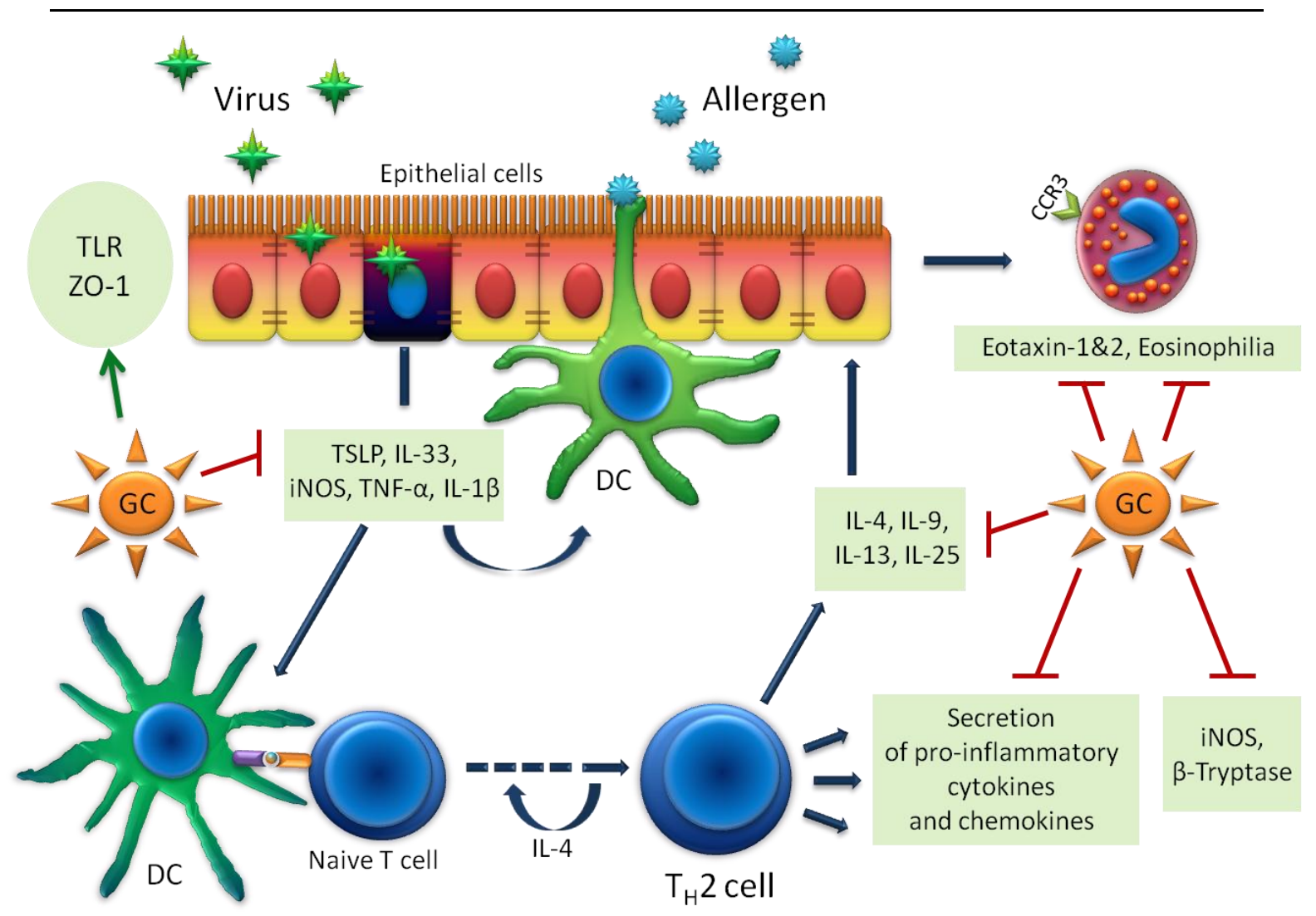

Graphical abstract: Mechanisms of asthma therapy with GCs. Airway epithelial cells express a variety of mediators involved in inflammation and host defense. Microbial or virus infection and epithelial damage in the respiratory airways can lead to the development of an asthmatic reaction. During the treatment of asthma GCs can suppress the larger part of pro-inflammatory mediators but only a suppression of a few of these cytokines/chemokines seems to be crucial for treatment efficiency. The schema visualizes the hypotheses of key mediator regulation by GCs. (Red arrows indicate the reduction of mRNA expression and mediator release. Green ones indicate the upregulation of TLR and ZO-1 expression by GC therapy. Blue arrows indicate the interaction between epithelial and immune cells).

Taken together the results of this work demonstrate that the suppression of AT2 cells and other structural cells of the lungs could be critical targets for asthma therapy by GCs and that the effects of GCs are mediated by the DNA-binding dependent mode of GR action. This information could become interesting for the development of new anti-asthmatic drugs that selectively inhibit crucial aspects of epithelial-immune or the epithelial-mesenchymal interactions. 
1. Introduction

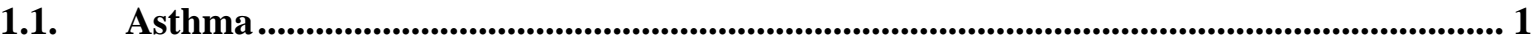

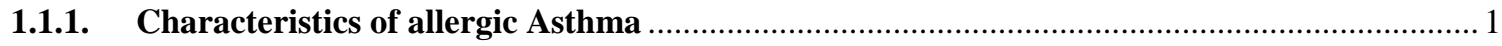

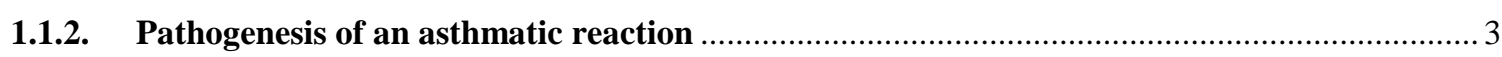

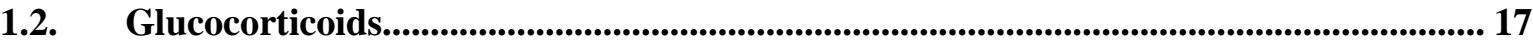

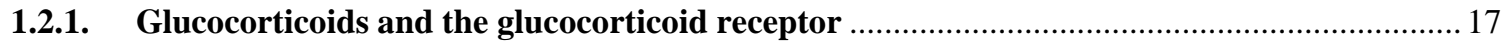

1.2.2. Therapeutical and side effects of asthma therapy with GCs................................................ 21

1.2.3. Effects of GC-therapy in murine models of inflammatory diseases ...................................... 23

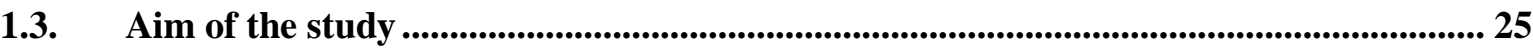

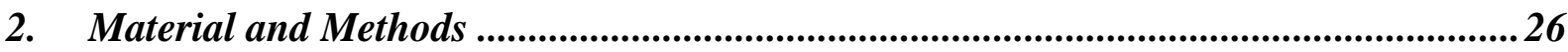

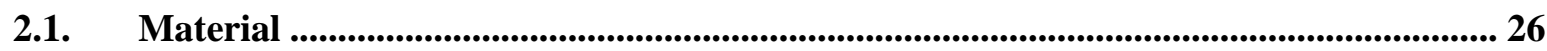

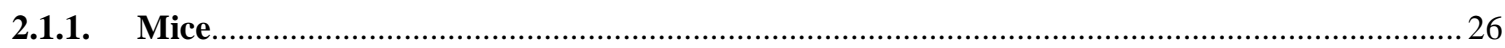

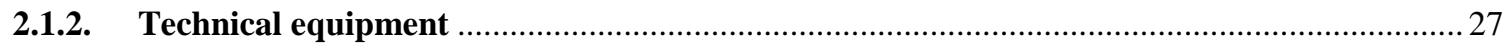

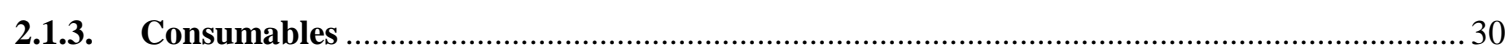

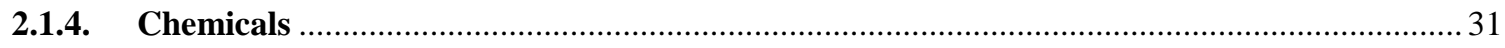

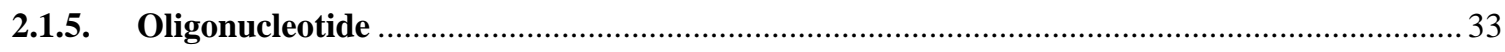

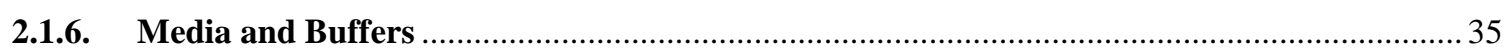

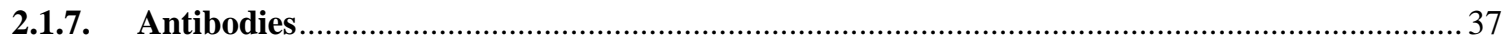

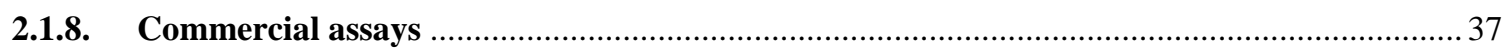

2.1.9. Software

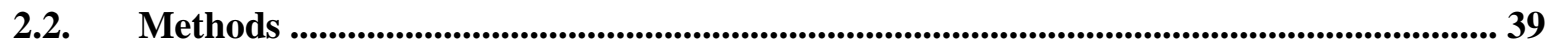

2.2.1. Animal work

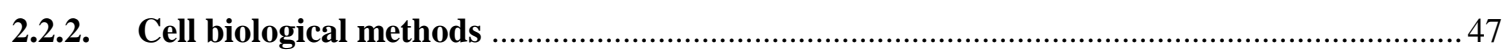

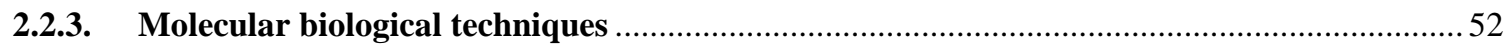

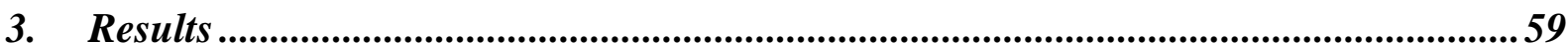

3.1. Establishment of AAI as a mouse model of human Asthma bronchiale........................ 59

3.1.1. Histological analysis of lung inflammation in wild type mice ................................................ 59

3.1.2. Establishment of the gating strategy for flow cytometric analysis of the BAL ....................... 61

3.1.3. Establishment of GC-therapy of AAI and mouse strain characteristics.................................. 62

3.1.4. Establishment of NIR imaging of Asthma in the mouse model of AAI ................................... 66

3.2. Identification of target cell populations of GCs by analyzing therapy efficiency in different GR-mutant mouse stains .................................................................................. 71

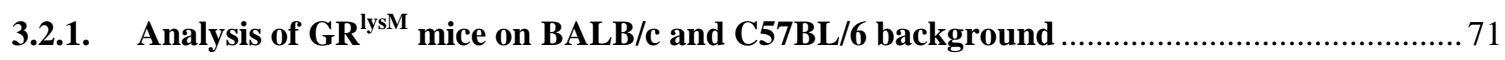

3.2.2. Analysis of AAI and treatability of $\mathrm{GR}^{\mathrm{lysM}}$ mice on a BALB/c background with GCs..........73

3.2.3. Analysis of AAI induction and the efficiency of GC-therapy inGR ${ }^{\text {lck }}, G^{\text {lysMlck }}$ and $G R^{\text {lysM }}$ mice on a $\mathrm{C} 57 \mathrm{BL} / 6$ background 
3.2.4. Analysis of AAI Induction and Treatability of GR $^{\mathrm{CD} 19}$ mice with GCs................................ 78

3.2.5. Analysis of AAI induction and treatability of $\mathbf{G R}^{\mathrm{CD11c}}$ and $\mathbf{G R}^{\mathrm{flox}}$ with GCs.......................... 81

3.2.6. Analysis of the AAI induction and treatability of $\mathrm{Kit}^{\mathrm{W-sh} / \mathrm{W-sh}}$ mice with GCs.......................... 84

3.3. The role of DNA-binding-dependent transactivation function of the GR for GC-

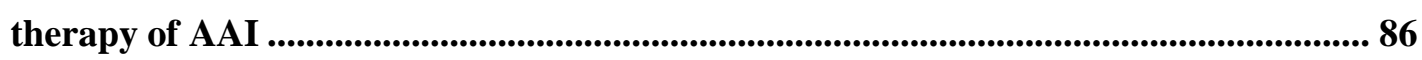

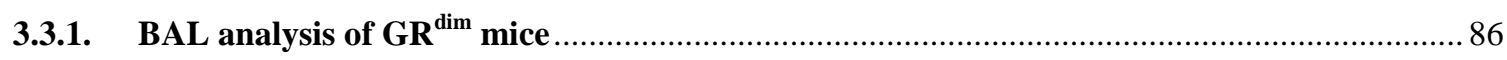

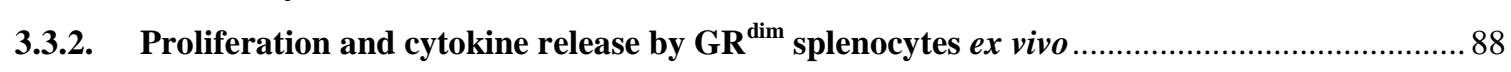

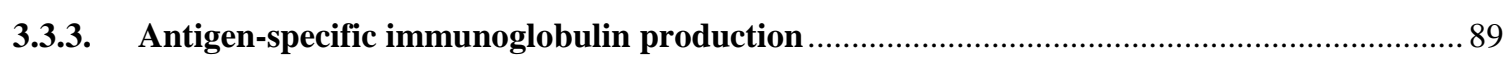

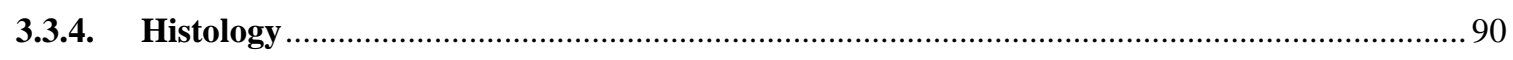

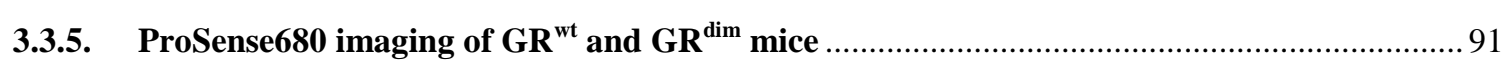

3.3.6. Detection of IL-5 secretion in serum samples of GR $^{\mathrm{dim}}$ mice ............................................... 92

3.3.7. Effects of GCs on the barrier function of the lung endothelium in $\mathbf{G R}^{\mathrm{dim}}$ mice with AAI ....92

3.3.8. Gene expression analysis of the BAL and Lung in GR $^{\mathrm{dim}}$ mice .......................................... 93

3.4. The role of non-hematopoietic cell types in the therapy of asthma with GCs............ 100

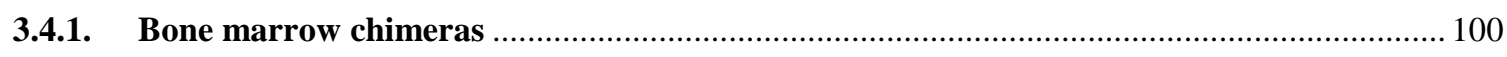

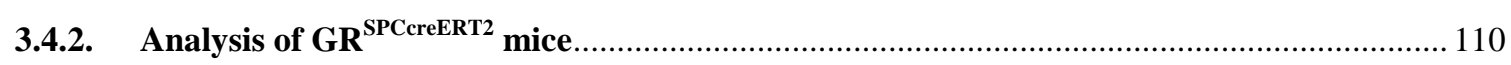

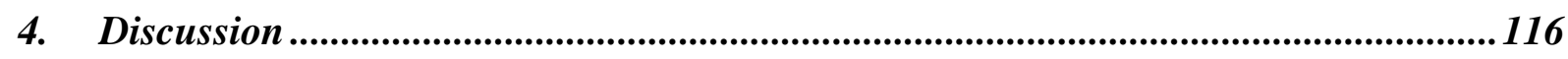

4.1. Asthma and the experimental mouse model of allergic airway inflammation ........... 116

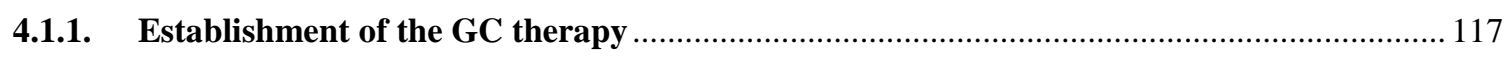

4.2. Role of GR DNA-binding and dimerization for GC-therapy of AAI as a model of Asthma ................................................................................................................. 118

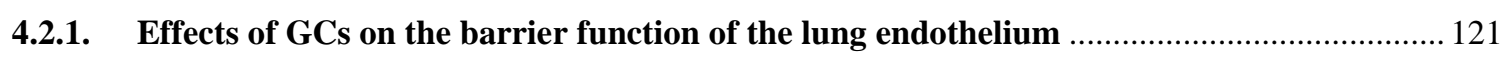

4.2.2. Effects of GCs on gene expression in BAL and lung parenchymal cells ............................. 121

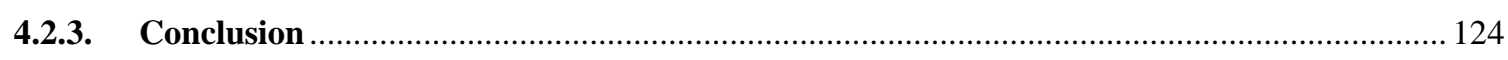

4.3 Identification of target cells of GCs therapy of asthma in the mouse model of AAI . 125

4.3.1. Effects of GC therapy on the regulation of gene expression in the lungs ............................ 126

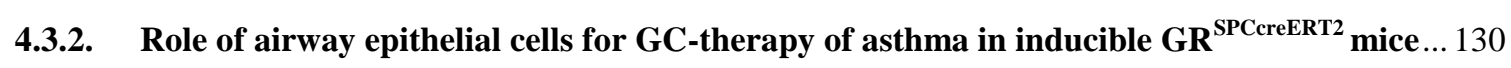

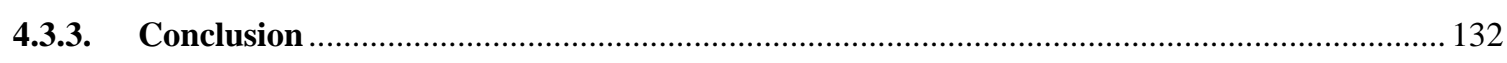

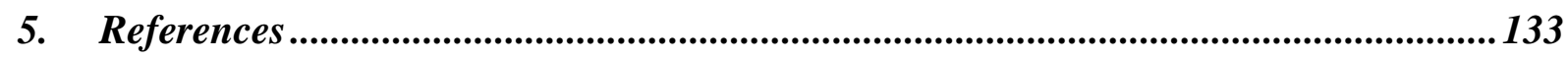

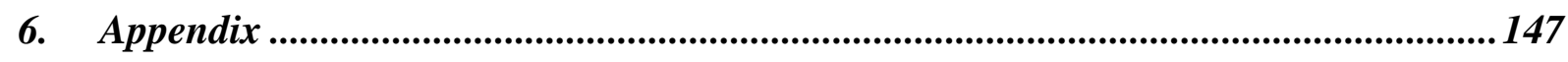

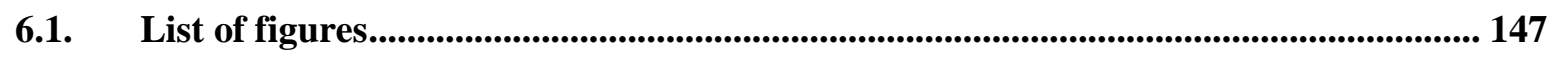

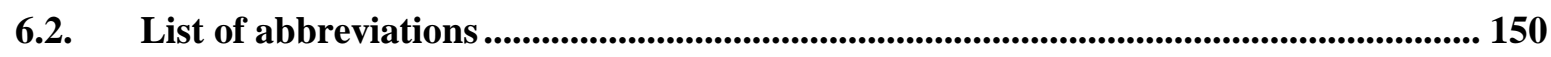

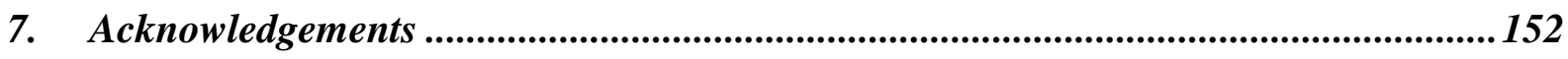


1. Introduction

\subsection{Asthma}

\subsubsection{Characteristics of allergic Asthma}

Asthma is a common chronic disorder of the airways that is characterized by airflow obstruction in association with hyperresponsiveness (AHR) and inflammation of the airways. These structural changes which are triggered by an immune response directed against inhaled antigens lead to various symptoms that are typically associated with asthma such as wheezing, breathlessness, chest tightness, and coughing (Holgate, 2013).

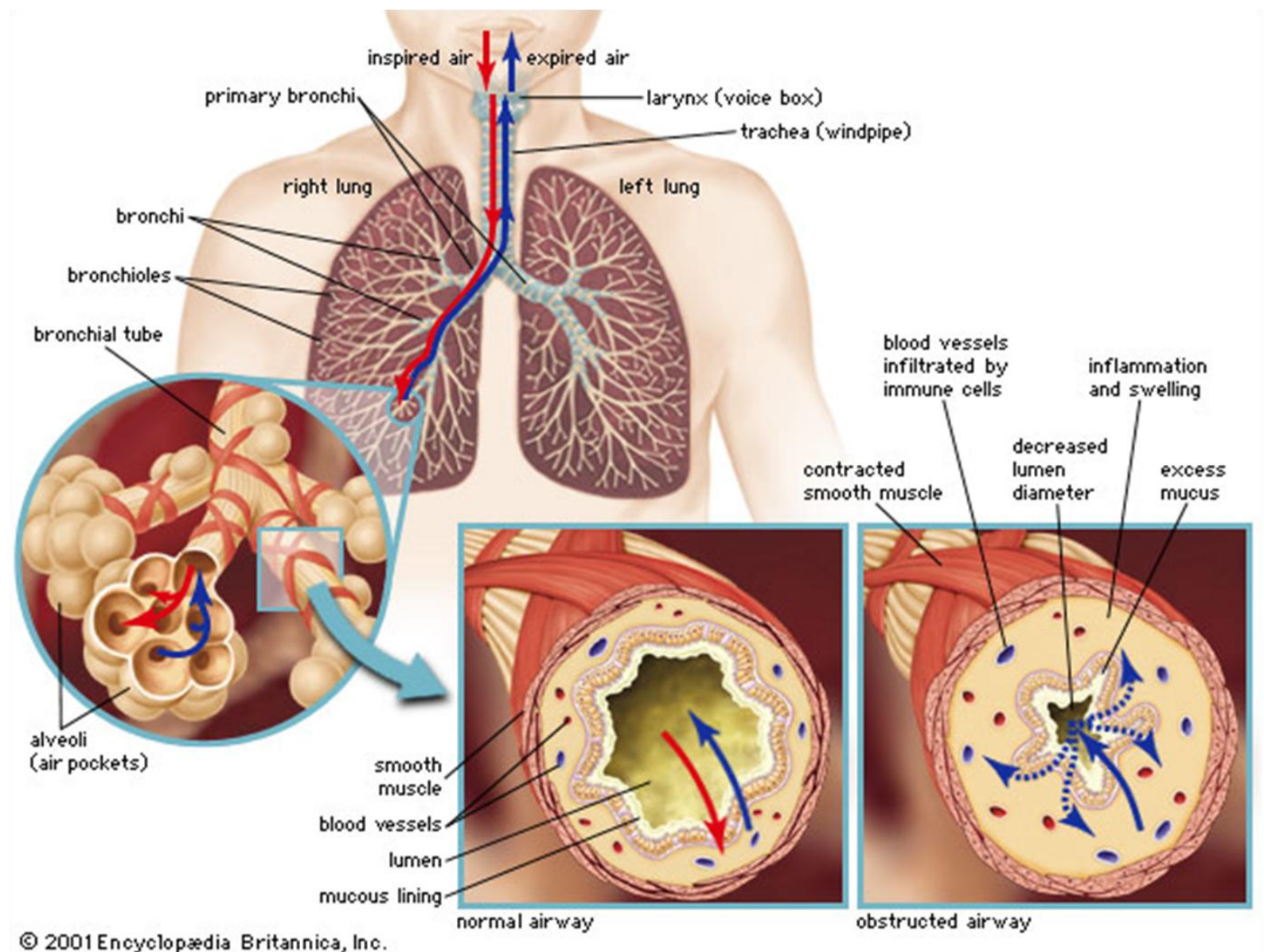

Figure 1: Anatomy of asthma attack. Asthma is characterized by a complex interaction of airflow obstruction, airway hyperresponsiveness and inflammation. The symptoms, which occur during an asthma attack, are spasmodic contraction of the airway smooth muscles, increased mucus secretion and infiltration by immune cells. These structural and cellular changes impede breathing to a greater or lesser extent. (Encyclopædia Britannica, Inc., 2001)

The pathophysiology of asthma and the corresponding dysfunction of the airways is based on the release of potent inflammatory mediators that recruit inflammatory cells on the one hand and cause airway wall remodelling on the other hand. 


\subsubsection{Cellular changes}

Airway inflammation in asthma is a multicellular process involving different cell types like T cells, mast cells, basophils, dendritic cells, B cells, macrophages and neutrophiles. However, the reaction is largely based on the development of a strong eosinophilia in the lungs. The distribution of infiltrating eosinophils differs between the peripheral and central airways and the lung parenchymal regions which may have important functional consequences. Interestingly, in patients with severe and steroid-dependent forms of asthma another type of the granulocyte population begins to infiltrate the inflamed lungs as well. In these patients a pronounced neutrophilia can occur instead of eosinophilia. This effect and its underlying mechanism is only poorly understood but can possibly be used as a marker for the severity of diesease progression or to evaluate the effects of long-term glucocorticoidtreatment during therapy.

\subsubsection{Structural changes}

The structural changes in the lungs of asthmatic patients can be characterized by an increase of airway wall smooth muscle mass, mucus gland hypertrophy and vascular congestion (Saetta and Turato, 2001). These features together can strongly contribute to the airflow limitations due to thickened airway walls with markedly reduced airway caliber and increasing airway resistance. Additionally, the difficulties with air uptake can be worsened by increased amounts of secreted mucus and infiltrating cells which blockade the free space in the conducting airways and cause an increased surface tension leading to airway closure. Mucus hypersecretion and the increase in smooth muscle mass, which are based on hypertrophy of mucous glands and goblet cells hyperplasia (Aikawa T. et al., 1992; Shimura S. et al., 1996) and strong proliferation of smooth muscle cells, respectively, are induced by inflammatory mediators (Noveral et al., 1992), cytokines (De et al., 1995) and growth factors (Noverale et al., 1992; Stewart et al., 1994) from the inflamed lung tissue and cellular infiltrates. Other prominent structural changes have been reported as well. These include vascular congestion with increased vessel area in the airway wall and subepithelial fibrosis with increased thickness of the reticular basement membrane and deposition of collagen types I, III and V (Roche et al., 1989). 


\subsubsection{Pathogenesis of an asthmatic reaction}

Although millions of people in the world are suffering from asthmatic symptoms, the precise cellular and biochemical processes underlying chronic inflammation and airway remodeling are poorly understood. Asthma usually develops in childhood and is associated with contact and sensitization to common aeroallergens derived from house dust mites, animal dander, fungi and pollen. The question why these harmless substances can induce strong inflammatory response in one part of population while they are tolerated by others has yet to be resolved.

One approach which was used to reveal risk factors for asthma development was a genomewide linkage screen of asthmatic and healthy persons in different populations to detect chromosomal regions that are linked to the disease. Several studies investigated this topic and a number of chromosomal regions have been reported to have a biological relevance for asthma and allergic diseases. These include the cytokine cluster on chromosome $5 q$ which harbors the genes encoding Interleukin (IL)-3, IL-5 and GM-CSF but also FCER1B on 11q, IFN- $\gamma$ and STAT6 on $12 q$ and IL-4R (the IL-4R $\alpha$ chain which is also a part of the IL-13R) on 16p (March et al., 2011).

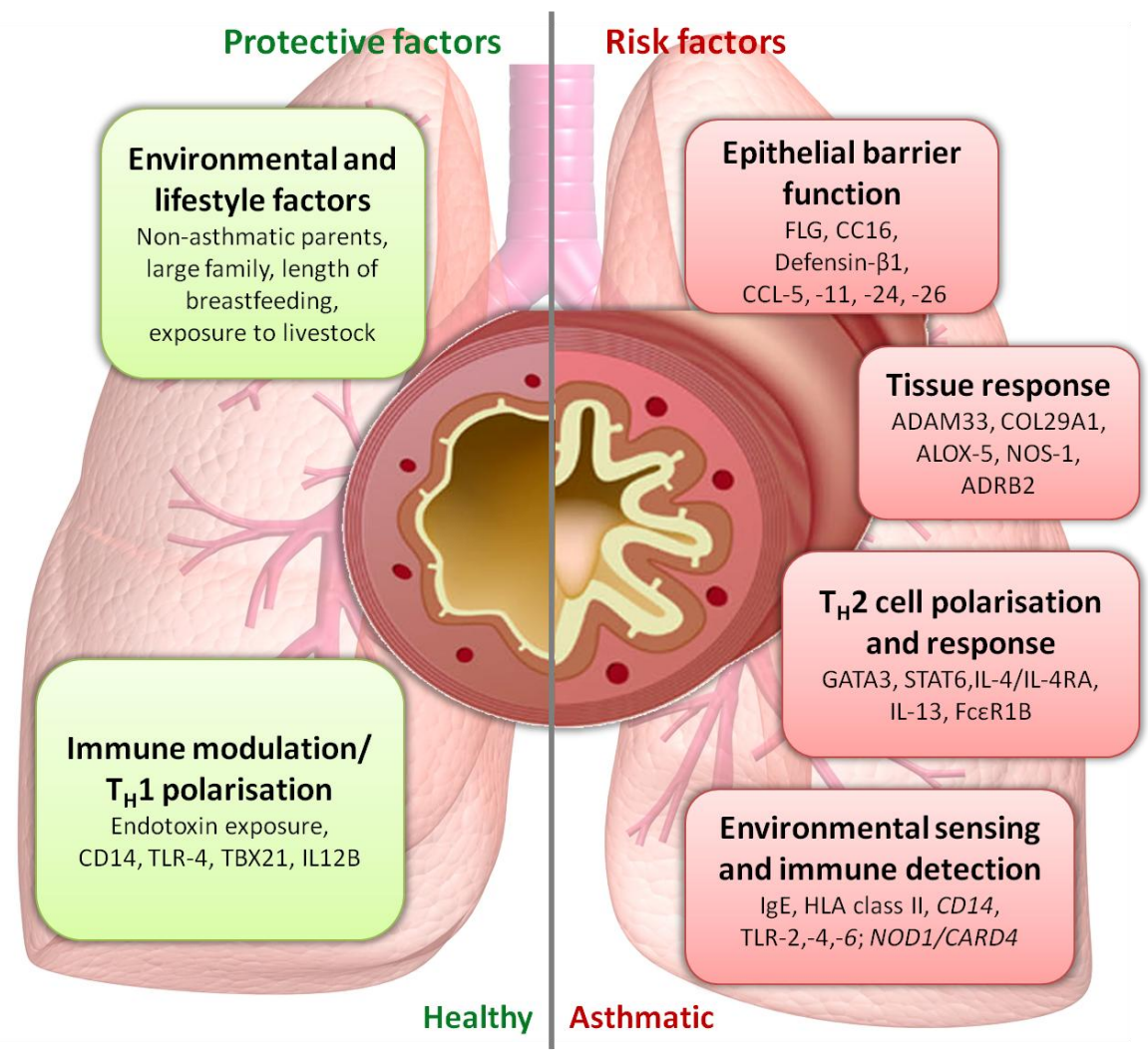

Figure 2: Protective and risk factors for asthma development identified with the help of genome wide association, linkage and candidate gene studies (modified and adopted from Michael E. March, 2011) 
Although there are many different studies investigating this topic (Ober and Hoffjan, 2006; Vercelli, 2008; Zhang et al., 2008), the identified "risk factor" genes can be categorized into the following groups: epithelial barrier function and innate immunity, tissue response, genes involved in $\mathrm{T}_{\mathrm{H}} 2$ cell polarization and genes critical for the cellular responses that characterize atopic disease and genes important for the environmental sensing and immune detection of microbial products (Figure 2). Mutations in these groups of genes are associated with an increased risk of asthma development (Michael E. March et al., 2011)

Unfortunately, very little is known about "protective factors" for asthma because this topic is less well explored. In the current literature, protective factors like healthy family members, exposure to pets and/or livestock and contact to farm animals have been proposed. The duration of breastfeeding or the exposure to endotoxins may also play an important role. The protective role of endotoxins such as LPS is presumably based on its binding to the CD14 molecule or interaction with TLR-4. Another factor that can suppress the development of asthma is an immune modulation inducing a $\mathrm{T}_{\mathrm{H}} 1$-biased response. Furthermore, the proteins TBX21 and IL-12B were reported to promote the development of $\mathrm{T}_{\mathrm{H}} 1$ cells which suppress atopic phenotypes (see Figure 2).

The current studies of risk and protective factors in asthma and allergy development underline the importance of the epithelial barrier function, of molecules involved in sensing of and effector function of innate immunity as well as the critical role of the $T_{H} 1 / T_{H} 2$ balance.

\subsubsection{Cellular mechanisms of asthma development}

The initial step which leads to an asthmatic reaction remains poorly understood. However, it is known that this process requires the presentation of allergenic peptides on MHC class II molecules together with co-stimulatory ligands to naïve T cells. This is performed by a special subset of cells, the so-called professional antigen-presenting cells (APCs) which also polarize the immune reaction in favour of a $\mathrm{T}_{\mathrm{H}} 2$ phenotype (Riffo-Vasquez and Spina, 2002; Pearlman et al., 1999).

APCs include B cells, macrophages and dendritic cells (DCs) with the latter being the most potent inducers of $\mathrm{T}$ cell responses. They are present in large amounts in the lung and are required to initiate and maintain an adaptive $\mathrm{T}_{\mathrm{H}} 2$ response to allergen. 
Like all other body surfaces, the lung is lined with an elaborate network of DCs which are dispersed throughout the conducting airways, lung interstitum, pleura, lung vasculature and bronchial lymph nodes (Geurts van Kessel and Lambrecht, 2008). The lungs DCs perform a sentinel function in the pulmonary immune response and act as a bridge between innate and adoptive immunity. They can also recognize inhaled antigens through expression of patternrecognition receptors such as Toll-like receptors (TLR), nucleotide-binding oligomerization domain (NOD)-like receptors, and C-type lectin receptors (Barrett et al., 2009). On the other hand, DCs react to mediators that are released upon damage of the tissue caused by pathogens, trauma, vascular damage or necrosis. Using these features, DCs can sense various danger-signals in the airways migrate to regional draining lymph nodes and process the relevant antigens (Vermaelen et al., 2001). In contrast to alveolar macrophages, DCs in the lung are reported to be very effective in generating $\mathrm{T}$ cell responses (Belz et al., 2004) and play a crucial role in initiating and perpetuating $\mathrm{T}$ cell hyperresponsiveness associated with asthmatic reactions (van Rijt et al., 2005; Hammad et al., 2010).

Several studies reported that the presence of DCs is essential for the development of a $T_{H} 2$ response in murine models of asthma (Kool et al., 2008a, 2008b) and that these cells are necessary for primary immune response to inhaled allergens. On the other hand, DCs also play a very important role in protective immunity and respiratory tolerance (Tournoy et al., 2006) where they lead to inhibition of T-cell activation and induction of regulatory $\mathrm{T}$ cells (Lambrecht et al., 2006).

Although DCs seem to be necessary for the induction of $\mathrm{T}_{\mathrm{H}} 2$ responses to many different antigens, other cell types do also act as APCs in the lung. These include alveolar macrophages, B cells (Holt 2000), epithelial cells or basophils (Sokol et al., 2009).

This heterogeneity of potential APCs correlates with the complexity of immune-challenging pathogens that enter the lungs but may also constitute a possible reason for the failure of respiratory tolerance in some cases.

Based on genetic studies, patient biopsies and new research data obtained from animal models it could be shown that many different factors contribute to asthmatic symptoms which include various cell types, cytokines, chemokines and other mediators. The list of main players which acting during the development of inflammatory response after antigen presentation consists of 
immune cells such as B cells, mast cells, eosinophils and $\mathrm{T}_{\mathrm{H}} 2$ cells but also non-immune cells like epithelial cells, fibroblast and smooth muscle cells of the lung.

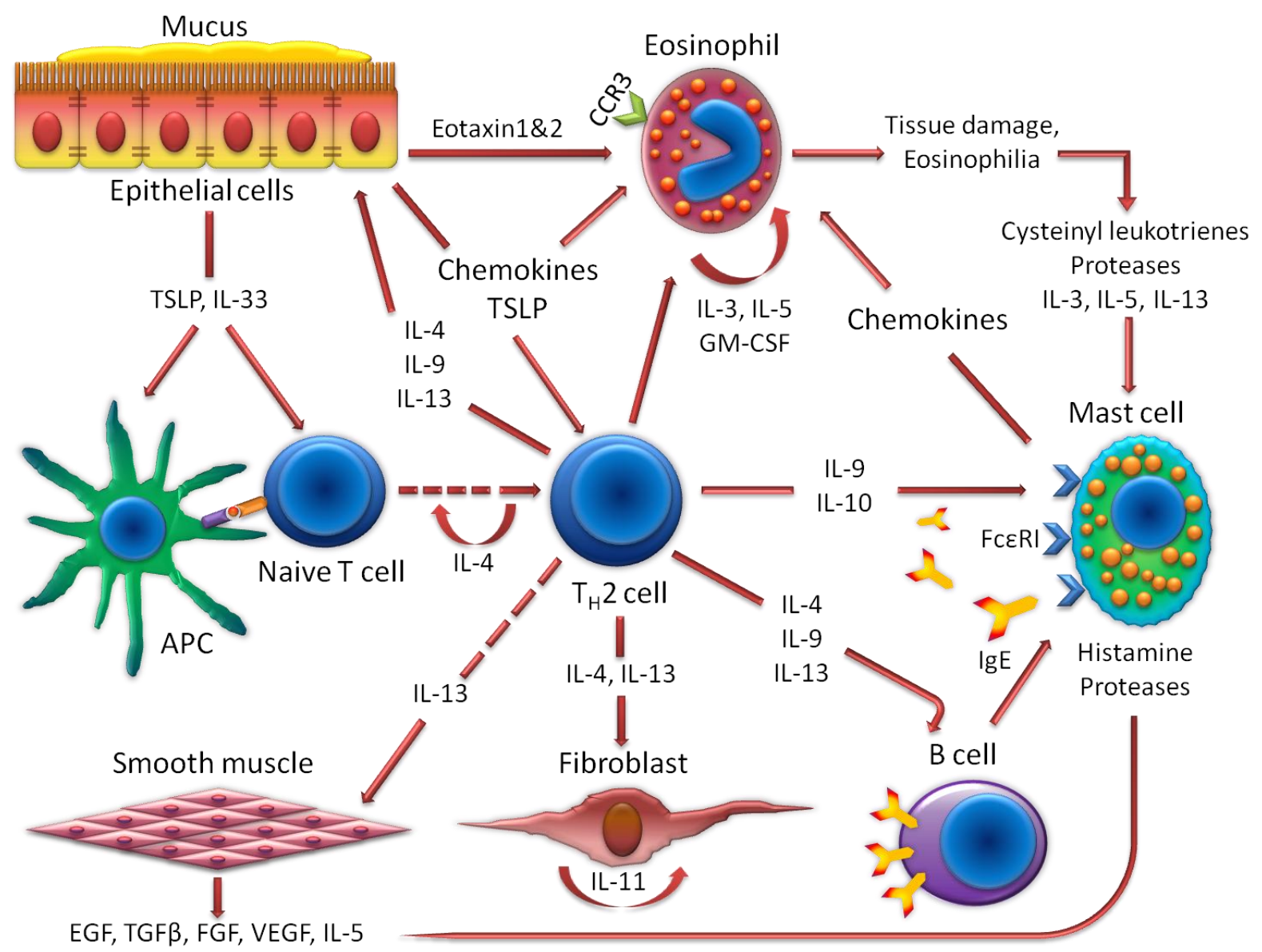

Figure 3: Cells and mediators involved in the induction of allergic asthma. The allergic response starts with antigen (Ag) sensitization, in which an antigen-presenting cell (APC) digests $\mathrm{Ag}$ and presents it to a naïve $\mathrm{T}$ cell on MHCII directing the differentiation in favour of a $\mathrm{T}_{\mathrm{H}} 2$ cell phenotype. $\mathrm{T}_{\mathrm{H}} 2$ cells produce various cytokines: IL-4, which promotes IgE synthesis via a B cell; IL-5, which promotes eosinophil maturation and activation; IL-9 and IL-13, which promote goblet cell hyperplasia and mucous secretion. Triggered by $\mathrm{Ag}-\mathrm{IgE}$ cross-linking, the mast cell releases histamine and proteases (leukotrienes and thromboxanes) that cause smooth muscle contraction and airway edema. Eosinophil-derived granule proteins lead to airway injury promoting activation of epithelial cells that is accompanied by chemokine and cytokine release (modified from Renauld et al., 2001; Jarjour et al., 2002 and Holgate et al., 2013).

The development of asthma generally starts largely with a selective expansion of $\mathrm{T}_{\mathrm{H}} 2$ lymphocytes that secrete a range of cytokines including interleukins IL-3, IL-4, IL-5, IL-9, IL-13 and granulocyte macrophage colony-stimulating factor (GM-CSF), which are tightly regulated (Holgate, 2012; Renauld et al., 2001). These $\mathrm{T}_{\mathrm{H}} 2$-specific cytokines orchestrate the allergic inflammatory cascade since they enhance survival of $\mathrm{T}_{\mathrm{H}} 2$ cells (IL-4), promote production of Immunoglobulin (Ig) E by B cells (IL-4 and IL-13) and lead to mast cell 
differentiation, maturation and activation (IL-3, IL-9, IL-13). In addition, they promote eosinophil maturation and survival (IL-3, IL-5 and GM-CSF) and cause hypersecretion of mucus through epithelial cells in the lung (Vignola et al., 2000; Vignola et al., 2003; Hammad et al., 2008; Holgate, 2013). The functions and the role of $\mathrm{T}_{\mathrm{H}} 2$ cells are closely connected with the functions of the produced cytokines (Figure 3).

Activated $\mathrm{T}_{\mathrm{H}} 2$ cells stimulate the production of $\operatorname{IgE}$ antibodies from B cells not only by secreting IL-4 but also through the cytokines IL-13 and IL-9, although the latter are less potent. IgE leads to mast cell activation via binding to the high affinity IgE receptors (FceRI) on the surface of mast cells and basophils. Cross-linking of $\operatorname{IgE}$ triggers the release of preformed vasoactive mediators such as histamine but also promotes the synthesis of prostaglandins and leukotrienes and the transcription of several cytokines. Following antigen contact, mast cells release the contents of their granula which leads to immediate hypersensitivity reactions like the rapid induction of mucosal oedema, mucus hypersecretion and smooth muscle constriction of the lungs. The activated mast cells also release chemotactic factors that contribute to the recruitment of inflammatory cells, particularly eosinophils, and produce a wide range of cytokines. These include IL-1, IL-3, IL-4, IL-5, IL-6, IL-8, IL-10, IL-13, IL-16, tumor necrosis factor beta (TNF- $\beta$ ) and transforming growth factor beta (TGF$\beta)$. In addition, several chemokines including IL-8, lymphotactin, CCL1 (TCA-3), CCL5 (RANTES), CCL2 (monocyte chemoattractant proteins, MCP-1) and CCL3 (MIP-1a) are secreted as well (Barret et al., 2009). It could be shown that these mast cell-derived mediators do not only play a role in cell recruitment but also induce activation of DCs and T cells (Robinson et al., 2004).

Even if the role of eosinophils in asthmatic reactions remains enigmatic, this cell population plays a prominent role in the initiation- and late phase of asthma pathogenesis. Eosinophilia has been described to be a major cause of tissue damage that occurs in the airways. Unlike mast cells, eosinophils do not express FceRI on their surface constitutivly but, once activated, start to express high levels of this receptor. Activation of the eosinophils causes the release of several mediators including major basic protein, eosinophil cationic protein and free radicals. These potent cytotoxic proteins and molecules not only lead to significant tissue damage because of their cytotoxic effects via osmotic lysis, but can also initiate the activation of mast cells and basophils upon degranulation. The accumulation of activated eosinophils in the lungs initiates the activation of other inflammatory cells which leads to the remodeling of the 
airways through hyperplasia and hypertrophy of the smooth muscle layer and mucous secreting glands. The combination of all these effects leads to reduced airways calibers (Renauld et al., 2001). Synthesis of prostaglandins, leukotriens and different cytokines including IL-5 acts as a positive feedback loop by activating additional eosinophils and enhancing infiltration. Interestingly, eosinophils play an important role in tissue damage during asthma pathogenesis, but are reported to be not required for allergen-induced airway hyperresponsiveness (Renauld et al., 2001)

In addition to cell types like eosinophiles which strongly enforce the pathogenic effects of inflammatory responses, there are also cell populations which do not only have proinflammatory but also anti-inflammatory functions. The alveolar macrophages play either an immunogenic or tolerogenic role depending on the signals received. Each of these characteristic may belong to a different macrophage phenotype with different functions in inflammatory responses (Stout et al., 2004; Mosser et al., 2008). Kreider and Martinez for example reported that the exposure of macrophages to IL-4 or IL-13 resulted in a population of anti-inflammatory macrophages which are involved in tissue repair responses (Kreider et al., 2007; Martinez et al., 2009). Additionally, these macrophages could produce IL-10, one of the most potent anti-inflammatory cytokines that can prevent inflammation (Mosser et al., 2008). Classically activated macrophages are also present in the lungs and besides their cytotoxic functions can also prevent the onset of allergic airway inflammation upon IFN $\gamma$ stimulation by $\mathrm{T}_{\mathrm{H}} 1$ cells (Korf et al., 2006; Tang et al., 2001).

It is not really clear how the different macrophage phenotypes (pro-inflammatory and antiinflammatory) impact the severity of asthma or the effectiveness of therapies but new investigations suggest that the development of GC-resistance in severe asthma may contribute to the activation of pulmonary macrophages (Balhara and Gounni, 2012; Yang et al., 2012).

Another cell type which are involved in asthmatic reaction are regulatory $\mathrm{T}$ cells ( $\mathrm{T}$ reg). Like anti-inflammatory macrophages, $\mathrm{T}$ reg cells play a key role in negative immune regulation by inducing immune tolerance to inhaled allergens ( $\mathrm{Wu}$ et al., 2008). $\mathrm{T}$ reg cells act during the initiation stage of an immune response and regulate the establishment of stable contacts between APCs and naive T cells. This cell population can inhibit APC activity, or prevent effector $\mathrm{T}$ cell development via different strategies. Human studies reported that asthmatic 
individuals have fewer functional $\mathrm{T}$ reg cells which underline their important antiinflammatory role in this context.

\subsubsection{Effects of $T_{H} 2$ mediators during airway inflammation}

The pathogenesis of asthmatic reactions is complex and multifactorial. During the development of airway inflammation, a variety of cell types interact with each other by releasing different mediators which exert anti-inflammatory and pro-inflammatory effects. From the first steps of inflammation on, $\mathrm{T}_{\mathrm{H}} 2$ cytokines play the key role in orchestrating the disease course and symptoms appearance.

One of the key $\mathrm{T}_{\mathrm{H}} 2$ cytokines in allergic reactions is IL-4. This mediator plays a crucial role not only during the priming of naïve $\mathrm{T}$ cells in favour of a $\mathrm{T}_{\mathrm{H}} 2$ phenotype (Herrick and Bottomly, 2003) but also regulates the isotype switching in B cells to IgE. Furthermore, it induces expression of MHCII and CD23 on APCs, of adhesion molecules on endothelial cells and stimulates chemokine production and activation of eosinophils and mast cells (Wills-Karp et al., 2000). Interestingly, the primary function of IL-4 is of central importance only at the beginning of an asthmatic reaction. After the initial priming steps, a strong IL-4 presence is important for the development of inflammation but it cannot induce airway hyperreactivity on its own (Renauld et al., 2001). Hence, it has been reported that IL-4 inhibition does not prevent the development of asthma in experimental models (Hogan et al., 1997; Renauld et al., 2001)

In contrast to IL-4, inhibition of another important $\mathrm{T}_{\mathrm{H}} 2$ cytokine, namely IL-13, completely blocks airway hyperreactivity in mouse asthma models although the signal transduction pathways of both cytokines overlap since IL-13 also binds to the $\alpha$ chain of the IL-4 receptor. Although IL-13 shares many functions with IL-4, based on recent publications, this cytokine plays a role not in the initial priming phase but also in the following steps. IL-13 overexpression induces strong inflammation with mucus hypersecretion, subepithelial fibrosis and eotaxin production which leads to eosinophil infiltration into the lungs. Because IL-4 and IL-13 are strongly related to each other concerning their functions, increased secretion of IL-5, eosinophilic infiltration and IgE production are only prevented in the absence of both mediators (Renauld et al., 2001). 
Another cytokine which is functionally related to IL-13 and IL-4 is IL-9. This cytokine is produced by $\mathrm{T}_{\mathrm{H}} 2$ cells and does not only stimulate cell-proliferation and prevent apoptosis, but is also involved in lung eosinophilia, mucus hypersecretion, Ig production, and pulmonary mastocytosis (Dong et al., 1999; Longphre et al., 2001; Louahed et al., 1995; Petit-Frere et al., 1993). It was reported that mucus hypersecretion by goblet cells could be directly stimulated with IL-9 independently of IL-13 in murine models of asthma (Townsend et al., 2000). On the other hand, IL-9 alone could not compensate for the function of other $\mathrm{T}_{\mathrm{H}} 2$ cytokines in the development of an asthmatic reaction.

IL-5 which was originally defined as a T-cell-derived cytokine that triggers antibody production in activated $\mathrm{B}$ cells is currently believed to play the crucial role in the development of eosinophilia in the lungs. As described in several publications, this cytokine is a key mediator of eosinophil differentiation, maturation, recruitment and activation at the sites of allergic inflammation (Humbert et al., 1997; Greenfeder et al., 2001). Furthermore, the high abundance of IL-5 mRNA in the sputum of asthmatic patients reflects eosinophil infiltration in the lungs (Truyen et al., 2006).

The thymic stromal lymphopoietin (TSLP) belongs to the key mediators of asthmatic reactions. This epithelial-derived cytokine does not only promote the development and migration of basophiles but is also associated with increased IL-4 and IL-13 production. Overexpression of TSLP in the lungs leads to spontaneous inflammation (Yoo et al., 2005). This strong influence on the immune response is probably based on DC stimulation. TSLP is able to upregulate expression of co-stimulatory molecules and $\mathrm{T}_{\mathrm{H}}$ 2-attracting chemokine ligands in DCs. Interestingly, TSLP is also reported to directly act on naïve CD4 ${ }^{+} \mathrm{T}$ cells to promote proliferation in response to antigen (Leonard 2002)

IL-25 (also known as IL-17E) is a member of the IL-17 superfamily. This cytokine is produced by $\mathrm{T}_{\mathrm{H}} 2$ cells, mast cells and epithelial cells. IL-25 has the ability to induce the expression of key mediators of asthmatic reactions such as IL-4, IL-5, and IL-13. Its overexpression leads to inflammation mediated by eosinophils and increased IgE production in a murine model of asthma (Fort et al., 2001). This function of IL-25 was also observable in human samples of $\mathrm{T}_{\mathrm{H}} 2$ cells (Wang et al., 2007). Because allergic airway inflammation can be effectively inhibited in murine models by antagonizing the function of IL-25, this cytokine 
belongs to a group of potential asthma therapy targets, and a blocking antibody is already in clinical development (Ballantyne et al., 2007).

IL-33 belongs to the earliest released signaling molecules during airway inflammation. The damage or activation of epithelial cells of the lungs leads to the upregulation of IL-33 expression. It was reported that IL-33 can be produced by epithelia and myeloid cells as an alarmin for the rapid induction of IL-13-driven immunity (Prefontaine et al., 2010). This cytokine has comparable effects to $\mathrm{T}_{\mathrm{H}} 2$ cytokines such as IL-5 and IL-13. IL-33 stimulates the inflammatory mediator release of T cells, eosinophils, mast cells, dendritic cells and basophils (Borish and Steinke, 2011)

In contrast to the other cytokines discussed above, IL-10 has inhibitory effects on the inflammatory response. This cytokine can suppress effector mechanisms and production of pro-inflammatory cytokines and chemokines by macrophages, eosinophiles and neutrophils. It could be shown that asthmatic patients express reduced levels of IL-10 (Takanashi et al., 1999), thus illustrating the important role of this cytokine in the regulation of inflammatory processes.

Chemokines also play important roles in asthmatic reactions because these mediators can direct the infiltration of leukocytes into the airways. In particular, Eotaxin 1\&2 (CCL11, CCL24), RANTES (CCL5) and MIP-1 $\alpha$ (CCL3), CXCL12 (SDF-1) as well as chemokine receptors and ligands like MCP-1, CXCR3 and CXCR4 have been identified as being important for the exacerbation of experimentally induced asthma.

Although overexpression of IL-13 alone is sufficient to induce an inflammatory response, it could be shown that airway and lung tissue eosinophilia is dependent on eotaxin expression. Eotaxin 1 and eotaxin 2 are strongly overexpressed in asthmatic lungs (Corrigan et al., 1999). These mediators have the ability to chemoattract and activate inflammatory leukocytes, particularly lymphocytes, monocytes, eosinophils and basophils, as well as some stromal cells such as endothelial and smooth muscle cells (Alam et al., 1997). Because only Eotaxin 1 and 2 bind specifically to the eosinophil receptor CCR3, only these chemokines can specifically regulate eosinophilia during asthmatic reactions (Ugoccioni et al., 1997; Heath et al., 1997). 
Another important chemokine that can bind to CCR3 and that is among the major CC chemokines induced during atopic (Alam et al., 1996) and nonatopic (Humbert et al., 1997) asthmatic responses in clinical asthma is RANTES (Tonnel et al., 2001). It was reported that RANTES has not only chemoattractant effects on mast cells, eosinophils, baseophils and Tcells but also enhances the production of $\operatorname{IgE}$ and $\mathrm{IgG} 4$ by activated B cells. The fact that the severity of asthma and atopy is associated with a polymorphism in the RANTES promoter (Fryer et al., 2001) demonstrated the importance of its regulation for asthmatic reactions (Schuh et al., 2003).

The regulation of chemokines does not only affect the cell migration into the lungs but also influences the inflammatory response type. MCP-1 can polarize the naïve $\mathrm{T}$ cells towards the IL-4-producing $\mathrm{T}_{\mathrm{H}} 2$ phenotype while MIP-1 $\alpha$ was reported to promote the $\mathrm{T}_{\mathrm{H}} 1$ response with increased secretion of IFNy and diminished production of IL-4. These effects on $T_{H} 1 / T_{H} 2$ balance have a strong influence on asthma progression.

CXCR3 is a chemokine receptor that is preferentially expressed on $\mathrm{T}_{\mathrm{H}} 1$ cells, epithelial cells and some endothelial cells. In previous studies it could be shown that this chemokine receptor is able to regulate leukocyte trafficking and promotes $\mathrm{T}_{\mathrm{H}} 1$ cell maturation that leads to the suppression of a $\mathrm{T}_{\mathrm{H}} 2$ response. In contrast to $\mathrm{CXCR} 3$, the CXCR4 chemokine receptor (specific for CXCL12) is strongly expressed in inflammatory tissues and on TH2 cells and nearly absent in healthy organs. Its ligand CXCL12 (or SDF-1) is one of the inflammatory cytokines that is strongly chemotactic for inflammatory cells such as macrophages. A small molecule inhibitor of CXCR4 (AMD3100) was reported to be able to reduce inflammation and AHR in mice with AAI. The inhibitory effects are probably associated with a reduction in $\mathrm{T}_{\mathrm{H}} 2$ cytokines (Lukacs et al., 2006). The possibility that CCL12 may also be involved in the mobilization of eosinophil progenitor cells from the bone marrow - since eosinophils express CXCR4 (Dorman et al,. 2005) - make this receptor-ligand pair interesting for the development of new anti-asthmatic therapeutics.

\subsubsection{Non-immune cells of the lungs in the context of an inflammatory response}

The role of structural elements of the lungs during asthmatic reactions was largely ignored for decades. However, new investigations in this field clearly demonstrated that, although asthma is a $\mathrm{T}_{\mathrm{H}} 2$-driven inflammatory response, innate immunity and particularly non-immune cells of 
the lungs like epithelial cells, smooth muscle cells or fibroblasts play key roles in disease initiation and progression.

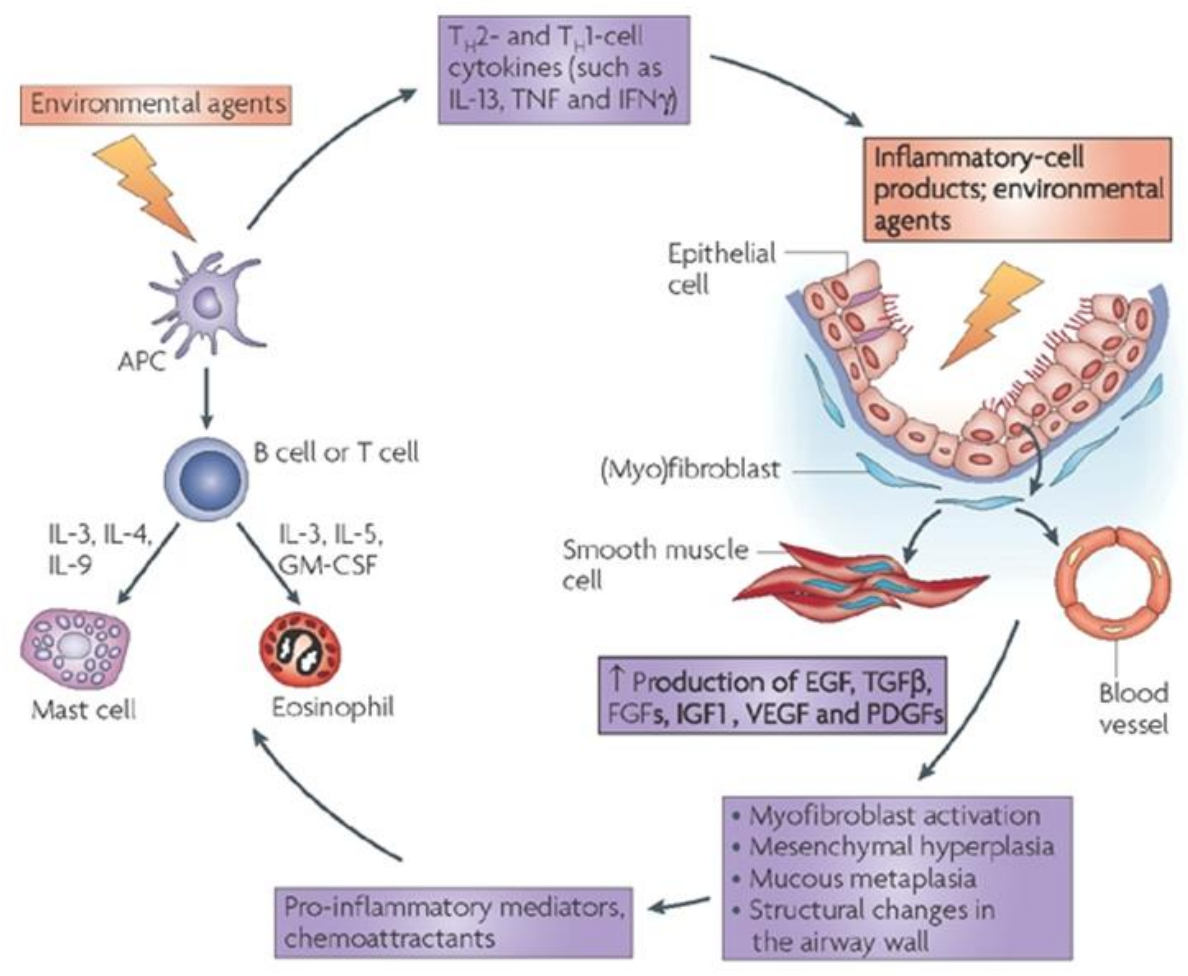

Nature Reviews | Immunology

Figure 4: The interaction between the immune system and structural cells of the lungs during an inflammatory response (Holgate and Riccardo Polosa, 2008)

Besides the primary function of the lung tissue which is to perform gas exchange, the lung cells are responsible for several important non-respiratory functions. Similar to other surface regions of the body, the lungs are in constant contact with the environment. Because of this, a variety of mechanisms developed in the lungs to avoid infection and to differentiate between pathogenic and harmless substances. This is achieved by downregulating or activating the non-specific and immune-mediated responses. The complexity of this sensitive coordination creates an immunocompetent and equally tolerant milieu to combat infections and to prevent collateral damage, respectively. The airway epithelium forms the first site of interaction between the internal milieu and the external environment. Because of this, the epithelial cells of the lungs continuously make contact with a variety of inhaled substances, in particular with airborne viruses, allergens, and environmental pollutants (Folkerts et al., 1998). The lung epithelium consists of many different cell types that allow gas-exchange, barrier-, regulatoryand defensive functions. 


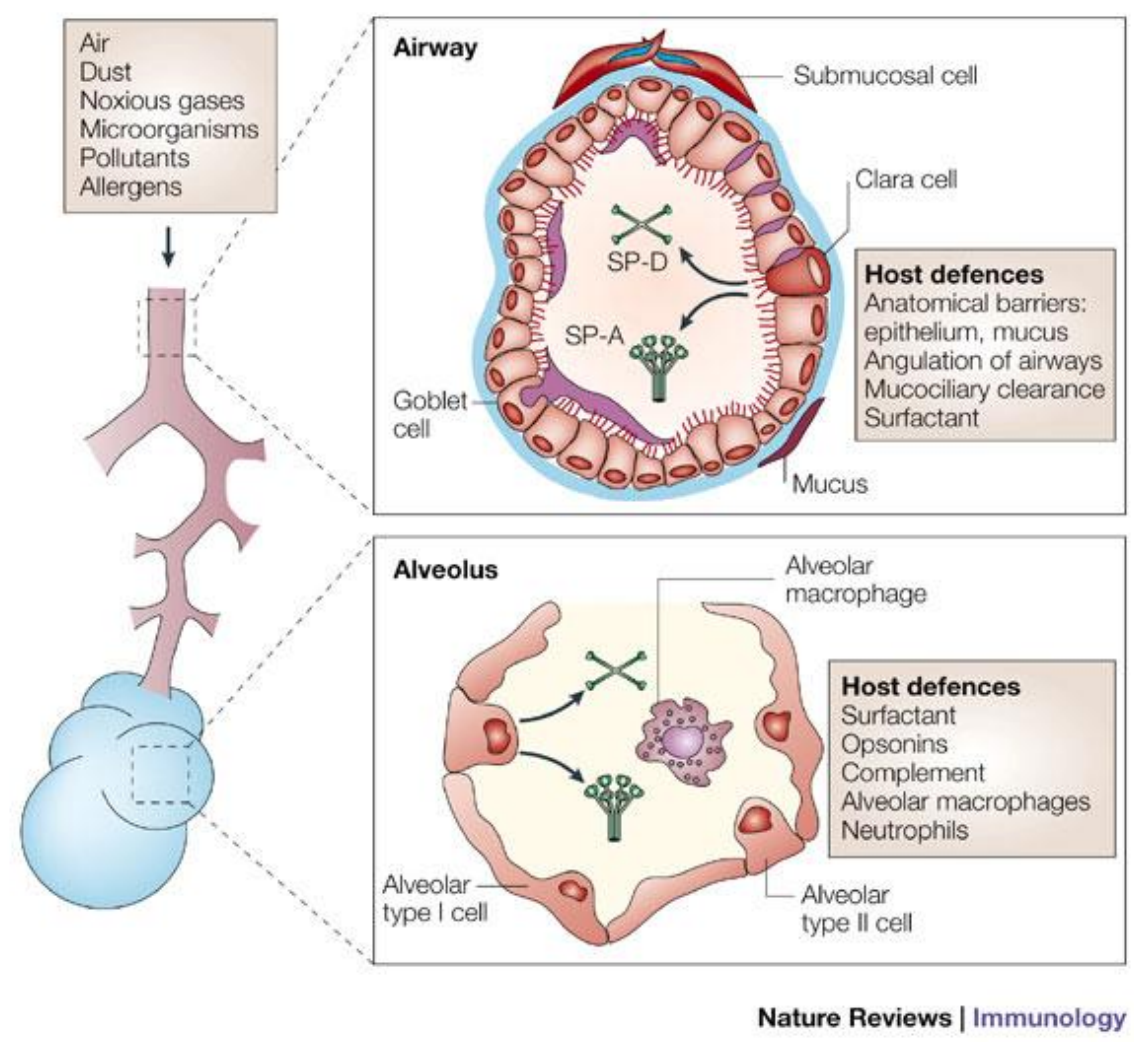

Figure 5: The mechanisms of host defense in airways and alveolus of the lungs. Because the lung is constantly challenged by inhaled pathogenic and harmless substances several mechanisms developed to perform defensive and regulatory functions (Wright et al., 2005)

About eight morphologically distinct epithelial cell types are present in the human respiratory epithelium. These cell types can be grouped into three different categories: basal-, ciliated and secretory epithelial cells (Spina et al., 1998). While ciliated cells transport the mucus from the lung to the pharynx (Harkema et al., 1991), mucus secreting goblet cells are responsible for efficient mucociliary clearance. In asthmatic reactions, epithelial cells upregulate the production of mucus in responses to $\mathrm{T}_{\mathrm{H}} 2$ cytokines such as IL-4, IL-13, and IL-9 which leads to goblet cell hyperplasia. The cells of the third group, the basal cells, belong to the primary stem cell type which can develop to mucus secretory or ciliated epithelial cell types. Besides the stem cell features, basal cells can secrete a number of bioactive mediators (Knight and Holgate, 2003). Particularly, Clara cells which are a subgroup of basal cells produce bronchiolar surfactant and specific proteases (De Water et al., 1985).

From an immunological point of view, the cells of the alveolar epithelium are in the focus of interest. The alveolar epithelium consists of two highly specialised cell types: alveolar type 1 and 2 cells (AT1 and AT2). While AT1 cells perform gas exchange functions, the AT2 cell population regulates a variety of processes including ion transport, surfactant metabolism, 
alveolar repair and immune modulation. Recent investigations demonstrate that AT2 can produce a number of pro-inflammatory cytokines upon activation. These consist of proinflammatory as well as regulatory mediators including arachidonic acid products, nitric oxide, endothelin-1, TGF- $\beta$, TNF- $\alpha$, and cytokines such as IL-1, IL-6 and IL-8 (Knight and Holgate, 2003). Surprisingly, the AT2 cells express MHCII molecules and can act as professional APC in the lungs directly influencing $\mathrm{T}$ cell priming and inflammatory responses (Holgate 2010).

The inflammatory reaction in the airways leads to a strong induction of growth factor release from epithelial cells such as TGF- $\beta$. This occurs in response to damage that directly influences the synthesis of matrix proteins by fibroblasts / myofibroblasts (de Boer et al., 1998; Vignola et al., 1997) but also their activation and proliferation. This fibroblast stimulation leads to airway remodelling and pulmonary fibrosis, the characteristically pathologic components of asthma development (Araya et al. 2010). The TGF- $\beta$ release from epithelial cells and fibroblast / myofibroblast influences not only airway remodelling and immune cell homeostasis (Takizawa et al., 2001, Redington et al., 1997) but also plays a crucial role in the induction of immunosuppressive $\mathrm{T}$ reg and pro-inflammatory $\mathrm{T}_{\mathrm{H}} 17$ cells $(\mathrm{Li}$ et al., 2008). This dual role of TGF- $\beta$ provides the opportunity to either promote inflammation with airway epithelial cell stimulation or to suppress inflammation with the help of $\mathrm{T}$ cells (Luo et al., 2010; Nakao et al., 2000). These features of fibroblast in communicating inflammatory signals correlate with their localization in the tissue which is between the epithelial cells and accumulating submucosal layer inflammatory cells such mast cells, eosinophils, DCs and T cells.

Another cell type that plays an important role in asthma pathogenesis are smooth muscle cells. In biopsies of asthmatic patients, the smooth muscle layer is strongly increased. These changes are based on smooth muscle cell hyperplasia, hypertrophy or increased extracellular matrix. On the other hand, airway smooth muscle (ASM) cells are biologically active and can produce a variety of mediators. ASM cells are able to promote migration of DCs and interact with mast cells (Saalbach et al., 2010, Schirmer et al., 2010). When exposed to IL-1 $\beta$, TNF- $\alpha$, IFN- $\gamma$, IL-4 or IL-13 in vitro, ASM cells are able to release inflammatory mediators such as IL-8, eotaxin, MCP-1, -2, and -3, GM-CSF, IL-5, RANTES, IL-6 and IL-11 (Ghaffar et al., 1999; Laberge et al., 1999). The investigation of bronchial biopsies of ASM cells from asthmatic patients also demonstrates the increased expression of RANTES and eotaxin which 
is indicative that these cells exhibit these mediator release also in vivo (Chung et al., 1999). Because these cytokines influence the migration and survival of eosinophils, mast cells, monocytes and other immune cells, the ASM cell population of the lungs may play a crucial role in inflammatory responses (Lazaar et al., 2001).

Taken together, the data about non-immune cells of the lung demonstrate new aspects of asthmatic reactions and complement the view of the mechanisms of disease development and progression. 


\subsection{Glucocorticoids}

\subsubsection{Glucocorticoids and the glucocorticoid receptor}

Glucocorticoids (GCs) are one of the most effective anti-inflammatory drugs available for the treatment of many autoimmune and inflammatory diseases including asthma. Although longterm therapy or high dose applications of these drugs are often associated with dramatic side effects, GCs have been used for many decades and remain the treatment of choice until today. GCs, such as the endogenous hormone cortisol or synthetic compounds like Dexamethasone, belong to the class of steroid hormones that exert their function through binding to the glucocorticoid receptor (GR).

The endogenous ligands of the GR are synthesized under the control of the hypothalamicpituitary-adrenal axis in the zona fasciculate and zona reticularis of the adrenal cortex and released diurnal in a pulsatile manner (Scheff et al., 2012). GCs are directly released into the circulation where they preferentially bind to their transporter protein transcortin (Richard et al., 2010).

The synthesis and concentration of GCs are tightly regulated by the hypothalamic-pituitaryadrenal (HPA) axis (Herman et al., 2003). Upon either inflammatory, physical or psychosocial stimulation, the cells of the paraventricular nucleus in the hypothalamus secrete corticotrophin-releasing hormone $(\mathrm{CRH})$. This, in turn, stimulates the anterior pituitary gland to release adrenocorticotropin hormone (ACTH) into the bloodstream. In the adrenal cortex, $\mathrm{ACTH}$ finally induces the synthesis and release of GCs (Figure 6). This way, the hypothalamus can influence the immune system through activation of the HPA axis, but the immune system can also influence the hypothalamus vice versa through cytokine release (Mulla and Buckingham 1999; Webster et al. 2002; Webster and Sternberg., 2004).

In addition to their effects on the immune system, endogenous GCs play an important role in the body's metabolism. Their functions include regulation of body growth and development, blood pressure, salt and water balance and energy metabolism (Andrews and Walker., 1999). As a result of GC effects on glucose metabolism, GC induce a state of insulin resistance, leading to diminished suppression of glucose production and reduced peripheral glucose uptake (Rizza et al., 1982, Macfarlane et al., 2008). In the immune system, GCs play a role in the regulation of lymphocyte apoptosis, $\mathrm{T}$ cell development and inflammatory responses. 


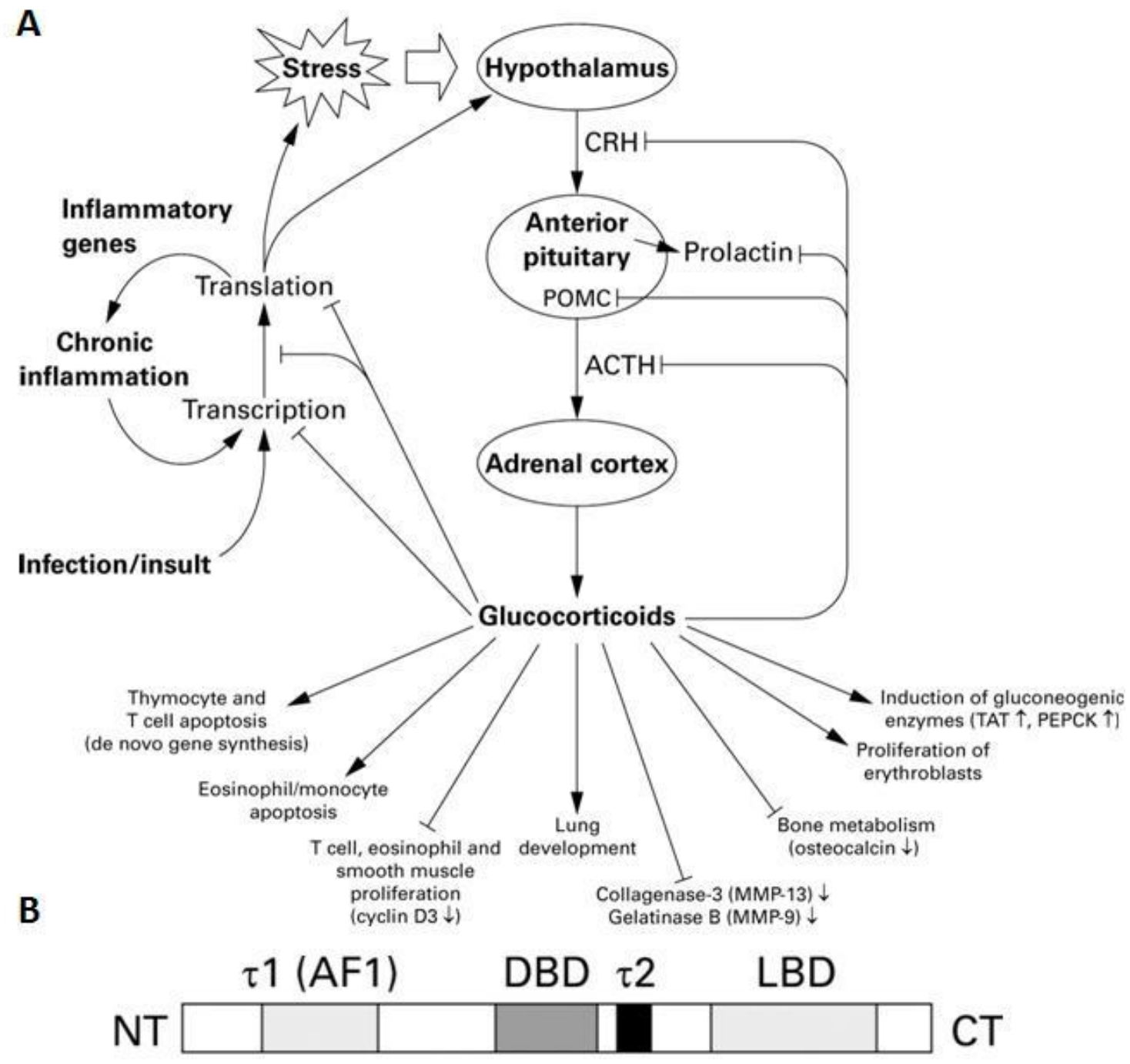

Figure 6: Effects of GCs on the HPA axis (A) and structure of the GR (B).

(A) This scheme shows the sites of synthesis and action of the main HPA hormones and the targets of GC action. Stress factors cause excitation of the hypothalamus, which responds by releasing CRH. This in turn acts on the anterior pituitary to induce synthesis and release ACTH which stimulates the adrenal cortex to release GC such as cortisol. (B) Linear representation of the 777 amino acid long sequence of the GR shows the principal domains. DBD = DNA binding domain; LBD = ligand binding domain; $\tau 1$ and $\tau 2=$ the two activation domains; $\mathrm{NT}=$ amino terminal; $\mathrm{CT}=$ carboxy terminal (adopted from Newton et al., 2000)

The GR, which is similar in structure to other nuclear receptors, is composed of several domains and subdomains. The six different domains can be classified in tree major functional units: the aminoterminal trans-activation domain AF1, the DNA-binding domain (DBD) and the carboxyterminal ligand-binding domain (Beato et al., 1995; Beato and Klug, 2000). The AF1 is a ligand-dependent activation domain that performs specific interactions with other transcriptions factors and components of the transcriptional machinery. The DBD consists of 
two zinc finger motifs and can recognize and bind to specific palindromic DNA-sequence motifs which are called GC response elements (GRE) (Luisi et al 1991; Umesono and Evans, 1989; Green et al., 1988). The sequence of the DBD contains five amino acids which are important for the formation of GR homodimers and their binding to the GRE (Beato et al., 1989; Luisi et al., 1991). The carboxyterminal ligand-binding domain consists of a ligandbinding site for specific and selective steroid hormone binding and a trans-activation domain with sequences important for dimerisation, nuclear localization as well as interactions with chaperons or other transcription factors (Beato and Klug, 2000).

The GR is present in almost every vertebrate cell. In the absence of hormone, the GR is localized in the cytoplasm where it is part of a large heteromeric complex (Morishima et al., 2003; Pratt and Toft, 2003). The role of this complex is to prevent the entering of the nucleus (Czar et al., 1995) and to support an optimal conformation for ligand binding. A number of chaperones act as subunits (hsp90, hsp70, hsp40) and cofactors (the co-chaperone p23; the immunophilins FKBP52 and Cyp40) (Gehring et al., 1993; Pratt et al., 1996). Because of the lipophilic character of GCs, these molecules can freely diffuse across the cell membrane and bind to different GC transporters and the GR. The binding of GCs to the GR induces a conformational change of the receptor resulting in disruption of the complex with chaperons and cofactors (Freedman et al., 2004). After the dissociation, the GR molecules become unmasked and translocate into the nucleus with the help of importin proteins (Freedman and Yamamoto. 2004; Tuckermann and Kleiman, 2007).

The GR influences inflammatory and metabolic processes by several mechanisms:

- non-genomic inhibition of signaling pathways

- $\quad$ DNA-binding dependent GR-mediated trans-activation of genes

- $\quad$ DNA-binding independent GR-mediated trans-repression of genes

Non-genomic mechanisms influence signaling pathways via direct interactions of the GR with signaling transduction components including the MAPK protein JNK and PI3K (Caelles et al., 1997; Kino et al., 2003; Limbourg and Liao, 2003). The effects of non-genomic GC action occur within minutes and are dependent on the production of second messenger molecules (Buttgereit and Scheffold, 2002) and activation of signal transduction pathways either by the cytosolic GR or by the membrane-bound GR (Stellato et al., 2004). 
Genomic mechanisms of GR action can be subdivided into two modes based on the binding partner for the GR and the effects on the gene expression. They are referred to as transactivation and trans-repression.

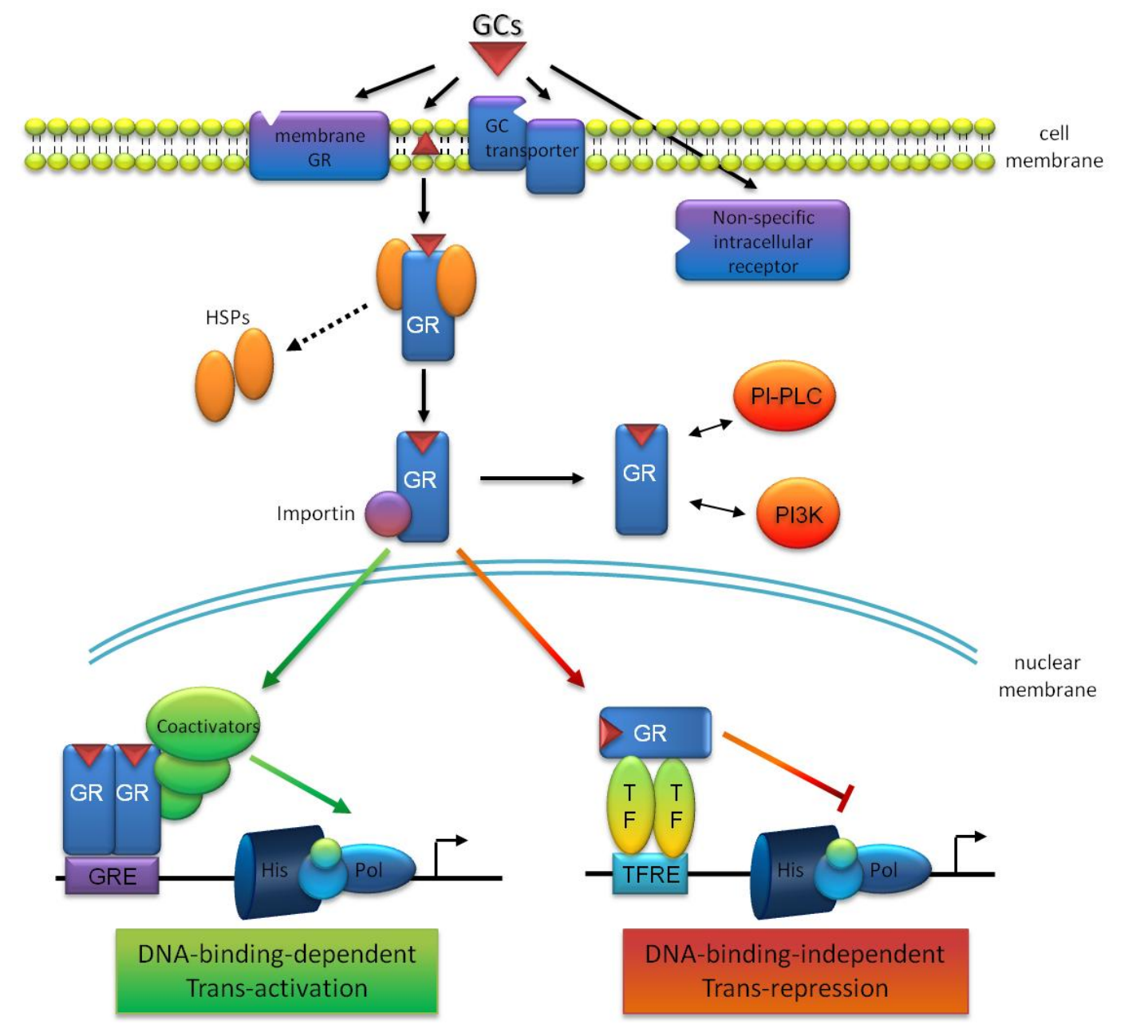

Figure 7: Mechanisms of GC action. GCs act through several modes of action: 1) non-genomic pathways through direct interactions of the GR signaling pathways. 2) DNA-binding dependent GRmediated transactivation of through binding of GR homodimers to GREs in the promoter region of target genes. 3) DNA-binding independent transrepression of genes through binding of the GR as monomers to DNA-bound transcription factors. GR: glucocorticoid receptor; GRE: glucocorticoid responsive element; TFREs: transcription factor responsive elements; HSPs: heat shock proteins; TF: transcription factor (adopted from Schweingruber et al., 2012)

The DNA-binding dependent mode of action occurs in a short period of time via binding of the GR as a homodimer to GREs (McNally et al., 2000; Metivier et al., 2003). The dimerization of the GR is realized with the help of distinct hydrophobic motifs in the ligand binding domain of each GR molecule (Bledsoe et al., 2002) and motifs present in the second zink-finger of the DBD. Furthermore, the DBDs N-terminal zinc finger motifs also directly contact the GRE of the DNA (Luisi et al., 1991; Cheung and Smith, 2000). After binding to the response element, the GR interacts with the basal transcription machinery and various 
cofactors to enhance the expression of the regulated gene. This process is part of the GR mode of action and called trans-activation. Through this mechanism, GCs can increase the production of different genes including the $\mathrm{NF} \kappa \mathrm{B}$ chaperone protein $\mathrm{I} \kappa \mathrm{B}$, dual specificity mitogen associated protein kinase phosphatase-1 (MAPK-1) (Kassel et al., 2001), glucocorticoid inducible leucine zipper (GILZ), lipocortin (annexin-1), IL-10, and surfactant protein D (Ito et al., 2006). It can also downregulate genes via negative gene regulation elements (nGREs) via this mechanism (Chambon et al., 2011).

The second genomic mechanism of GR action is DNA-binding independent. The so-called trans-repression is based on GR-mediated inhibition of other transcription factors. In this mode of action, the GR monomer suppresses gene expression without dimerization (Reichardt et al., 1998) via direct interaction with inflammatory transcription factors such as NF-кB (Reichardt et al., 2001), AP1 (Tuckermann et al., 1999) and Stat proteins (Tronche et al., 2004). The GR binds to the DNA-bound transcription factors and influences their transcription via direct inhibition, stimulation of chaperone production, co-factors inhibition or epigenetic mechanisms (Barnes et al., 2003; Stahn et al., 2007; Adcock et al., 2008; Ito et al., 2006; Löwenberg et al., 2008). Additionally, GCs also have post-transcriptional effects and are able to decrease the stability of some pro-inflammatory mRNA species (Barnes 2011).

\subsubsection{Therapeutical and side effects of asthma therapy with GCs}

After the establishment of GCs as a potent anti-inflammatory substance in the late 1940s, systemic treatment with GCs was introduced in the therapy of asthma. Today, millions of asthmatic patients are treated daily with different inhaled corticosteroids that have been in use for over 35 years. The therapy of asthma with GCs is very efficient and can strongly reduce asthmatic symptoms. GCs reduce airway inflammation, airway hyperresponsiveness, allergeninduced peribronchial fibrosis (Miller et al., 2006) but also the frequency of exacerbations, the production of $\mathrm{T}_{\mathrm{H}} 2$ cytokines and can even prevent irreversible airway changes (Barnes et al., 1998; O'Byrne et al., 2006; Barnes 2011).

At the cellular level during asthmatic reactions, GCs were reported to strongly reduce the number of inflammatory cells in the airways including eosinophils, $\mathrm{T}$ cells, mast cells and DCs (Barnes et al., 1998). These effects of GCs can be achieved through the suppression of cytokine and chemokine secretion as well as adhesion molecule expression and by inhibiting 
the survival of inflammatory cells in the airways (Figure 8). This inhibition of inflammatory cell recruitment and induction of apoptosis successfully leads to a reduced level of inflammation.

Although great efforts have been made to structurally modify GCs in order to develop more efficient and safe anti-asthmatic drugs, long-term therapy with GCs unfortunately is still accompanied by dramatic side effects. These include problems with glucose (Pidala et al., 2011) and amino acids metabolism, diabetes, osteoporosis (Canalis et al., 2007), cataract, hypertension and immunodeficiency (Barnes et al, 2010). Recent studies identified a correlation between positive and negative GC-effects and the mode of GC action. Based on these findings, transactivation is believed to be largely responsible for the reported side effects while the trans-repression activity of GCs is mostly associated with their beneficial therapeutic effects.

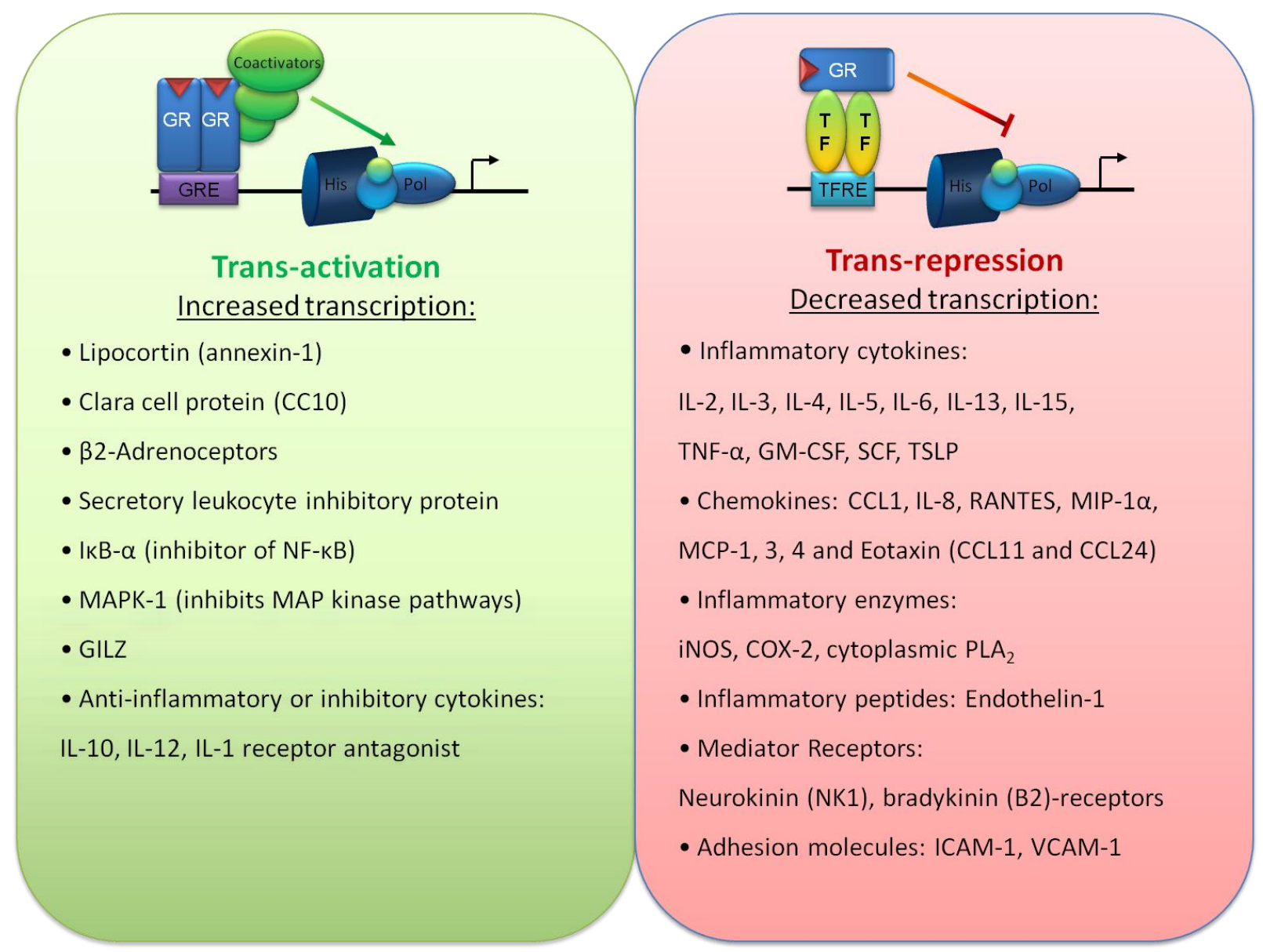

Figure 8: Effects of trans-activation and trans-repression on the expression of anti-inflammatory and pro-inflammatory mediators. There are indications that the beneficial effects of GC therapy are mainly mediated through transrepression, whereas side-effects correlate with transactivation (adopted from Barnes, 2011) 


\subsubsection{Effects of GC-therapy in murine models of inflammatory diseases}

The correlation between trans-repression and suppression of inflammation and immune activity opens the possibility to improve treatment efficacy and reduce side-effects using GCs without trans-activation activity, so-called dissociated GCs. In order to investigate this possibility and to study the molecular mechanism and function of GR action in detail, mouse mutants with different types of GR mutations were developed (Cole et al., 1995; Reichardt et al., 1998; Tronche et al., 1999; Baschant et al., 2011).

Mice completely lacking a functional GR are not able to survive and die shortly after birth (Cole et al., 1995; Tronche et al., 1998) which makes this model unsuitable to analyse GR function in vivo. To investigate the molecular mechanism of $\mathrm{GC}$ action in vivo and to dissect the effects of trans-activation and trans-repression, the $\mathrm{GR}^{\mathrm{dim}}$ mouse was developed (Reichardt et al., 1998). This mouse express a genetically modified form of the GR (Reichardt et al., 1998) that can only act via the DNA-binding independent mode of GR action because of a point mutation in the dimerization domain of the GR that effectively prevents GR dimerization. These mice survive until adulthood and do not display any problems in metabolic processes.

The GR ${ }^{\text {dim }}$ mouse opens up the possibility to test the concept concerning the mechanisms underlying the therapeutical and side effects which occur during GC-therapy. Analysis of therapeutical efficiency of GCs in GR ${ }^{\text {dim }}$ and wild type mice in a murine model of irritative skin inflammation demonstrated that suppression by GCs was intact in GR ${ }^{\mathrm{dim}}$ mice (Reichardt et al., 2001). On the other hand, GR ${ }^{\text {dim }}$ mice with a contact hypersensity reaction (CHS, Tuckermann et al., 2007), septic shock (Bhattacharyya et al., 2007; Kleiman et al., 2011) or antigen-induced arthritis (Baschant et al., 2011) demonstrated strongly reduced GC efficacy. These data show the dependency of GC-therapy efficiency on the inflammatory reaction and disease type (Baschant et al., 2011). 


\begin{tabular}{|c|c|c|}
\hline Therapeutic effects & Wild type mouse & GRdim mouse \\
\hline $\begin{array}{c}\text { Treatment of irrigative } \\
\text { dermatitis }\end{array}$ & + & + (Reichardt et al., 2001) \\
\hline Treatment of $\mathrm{CHS}$ & + & Impaired (Tuckermann et al., 2007) \\
\hline $\begin{array}{l}\text { Suppression of } \\
\text { inflammatory mediators }\end{array}$ & + & $\begin{array}{l}+ \text { : TNF- } \alpha \text {, MMP-13, MMP-9 } \\
\text { (Tuckermann et al., 2007) } \\
\text { Impaired: MCP-1, IP-10, IL-1 } \beta \\
\text { (Grose et al., 2002; Tuckermann et } \\
\text { al., 2007) }\end{array}$ \\
\hline Side effects & Wild type mouse & GR dim mouse \\
\hline $\begin{array}{l}\text { Induction of catabolic } \\
\text { enzymes }\end{array}$ & + & $\begin{array}{l}\text { Impaired : TAT, PEPCK (Reichardt et } \\
\text { al., 1998) }\end{array}$ \\
\hline GC induced osteoporosis & + & + (Tuckermann et al., 2010) \\
\hline
\end{tabular}

Figure 9: Therapeutic effects and side effects of GC-treatment in wild type and $\mathbf{G R}^{\mathrm{dim}}$ mice. To dissect the trans-activation dependent from trans-repression dependent effects of GR action, the GR ${ }^{\mathrm{dim}}$ mouse was analyzed in different disease models (adopted from Tuckermann and Kleiman., 2007).

Because the therapeutic efficiency in different disorders was found to be cell type specific (Baschant et al., 2011), the mechanism of GC-therapy is not the same for the treatment of all inflammatory disorders. While GC-therapy in murine models of contact hypersensitivity and septic shock is dependent on the suppression of myeloid cells, GC treatment of experimental autoimmune encephalomyelitis (EAE) exclusively required repression of $\mathrm{T}$ cell function (Wüst et al., 2009; Schweingruber et al., 2012). These new findings concerning the target cells of GC-therapy were obtained using different cell-type specific GR knockout mice. Those included mice lacking GR expression in myeloid cells (GR ${ }^{\text {lysM }}$, Tuckermann et al., 2007), in T cells (GR ${ }^{\text {lck }}$, Baumann et al., 2005), in T cells and myeloid cells (GR $\left.{ }^{\text {lcklysM }}\right)$, DCs $\left(\mathrm{GR}^{\mathrm{CD} 11 \mathrm{c}}\right.$, Caton, Smith-Raska and Reizis 2007) or B cells (GR ${ }^{\mathrm{CD} 19}$, Rickert, Roes and Rajewsky 1997). 


\subsection{Aim of the study}

GCs are the measure of choice for the treatment of inflammatory conditions such as asthma. To improve therapy new insights into the mode of action and target cells of GCs are needed. For this aim a mouse model of AAI should be analysed in this work.

It is well documented that most GC effects are mediated by the GR via transactivation or transrepression. The first mode of action requires dimerization of GR, its binding to regulatory DNA elements and subsequent activation of target genes. Alternatively, GR may control transcription in a DNA binding-independent manner, which is mediated by tethering interactions with other transcription factors such as AP-1 and NF- $\kappa \mathrm{B}$. While the first mechanism is thought to account for many side effects of GC, the second one presumably underlies the majority of its anti-inflammatory actions. To determine the requirement of both GR functions for GC intervention of asthma, $\mathrm{GR}^{\mathrm{dim}}$ mice which express a modified form of the GR that is no longer able to dimerize and therefore lack DNA-binding dependent gene regulation by GR should be analyzed. To this end the treatability should be compared with wild type mice using various experimental approaches.

Since the GR is present in almost every vertebrate cell including immune cells and structural cells of the lungs, it is difficult unclear which cells are important for the anti-inflammatory effects of GCs during asthma therapy. Another aim of this work was therefore to identify the essential target cells of GCs. To answer this question, mast cell deficient mice (cKit) and several cell-type specific GR-deficient mice which lack GR in T cells $\left(\mathrm{GR}^{\mathrm{lck}}\right)$, myeloid cells $\left(\mathrm{GR}^{\mathrm{lysM}}\right), \mathrm{DC}\left(\mathrm{GR}^{\mathrm{CD11C}}\right)$ or B cells $\left(\mathrm{GR}^{\mathrm{CD} 19}\right)$ should be used to investigate the role of different immune cells and non-immune cells for the suppression of asthma by GCs.

To further investigate the role of GC effects on non-immune cell populations of the lungs $\mathrm{GR}^{\mathrm{dim}}$ bone marrow chimeras and $\mathrm{GR}^{\mathrm{SPC} \text { SreERT2 }}$ mice with an inducible GR knockout in AT2 cells were analyzed.

Treatment of the different mutant mice with GCs was thought to reveal differences in the immunosuppressive effects compare to wild type mice and to provide more information about the mechanisms of GC-treatment of asthma. The animals should be analyzed by a series of methods including histology, ELISA, FACS and gene expression profiling to investigate GC effects in specific cell types. The resulting findings should help to identify the molecular and cellular mechanisms of GC action in the therapy of asthmatic reaction. 


\section{Material and Methods}

\subsection{Material}

\subsubsection{Mice}

All mice which were used during this work were kept under specific pathogen-free conditions in individually ventilated cages with unlimited access to the food and water, under a 12 hour day-night cycle. Wild type $\mathrm{BALB} / \mathrm{c}$ and $\mathrm{C} 57 \mathrm{Bl} / 6$ mice were obtained from Charles River Laboratories (Wilmington, USA).

To determine the mechanism of GC-treatment and the dependency of GC-therapy on transactivation vs. transrepression, $\mathrm{GR}^{\mathrm{dim}}$ mice were used (Reichardt et al., 1998), which express a modified form of the glucocorticoid receptor that is no longer able to dimerise and therefore lack DNA-binding dependent gene regulation by the GR. These mice were backcrossed to the BALB/c background for more than 10 generations and bred in our own animal facility in Göttingen.

Because the GR is present in almost every vertebrate cell, it is difficult to determine the crucial target cells, which are important for anti-inflammatory effects of GCs during asthma therapy. To identify essential target cells of GCs we therefore used cell-type specific GRdeficient mice which lack the GR in T cells $\left(\mathrm{GR}^{\text {flox/flox }}\right.$; lckCre (designated $\mathrm{GR}^{\text {lck }}$ ) Baumann $\mathrm{S}$, et al. (2005)), myeloid cells (GR ${ }^{\text {flox/flox}}$; lysMCre (designated $\mathrm{GR}^{\text {lysM }}$ ) Tuckermann JP, et al. (2007), in T cells and myeloid cells ((GR flox/flox ;ysMCre; lckCre (designated GR $\left.{ }^{\text {lcklysM }}\right)$, DCs $\left(\left(\mathrm{GR}^{\text {flox/flox }} ; \mathrm{CD} 11 \mathrm{cCre}\right.\right.$ (designated GR ${ }^{\mathrm{CD} 11 \mathrm{C}}$, Caton ML, Smith-Raska MR, Reizis B (2007)) or B cells ((GR flox/flox; CD19Cre (designated GR ${ }^{\mathrm{CD} 19}$, Rickert RC, Roes J, Rajewsky K (1997). These mice were generated by crossing GR ${ }^{\text {flox/flox }}$ mice with lckCre, lysMCre, CD11CCre or CD19Cre transgenic/knockin mice, respectively, and back-crossed for at least four generations to the C57BL/6 background.

The $\mathrm{GR}^{\text {lysM }}$ mice on a BALB/c background were generated at the Leibniz Institute for Age Research by crossing them for at least four generations to the BALB/c background. These mice were used to analyze GR expression on eosinophils and to analyze the lack of the GR on myeloid cells. These mutants were kindly provided by our co-operation partner Jan Tuckermann from the Leibniz Institute for Age Research in Jena. 
$\mathrm{Kit}^{\mathrm{W}-\mathrm{sh} / \mathrm{W}-\mathrm{sh}}$ (in the following text named as cKit) mice have been described previously (Grimbaldeston et al., 2005) were kindly provided by Professor Mauerer (Charite Berlin). These mice have a constitutive abolishment of mast cells in all tissues. Because of this feature, these animals can be used in experiments to analyze the dependence of GC effect on mast cells during asthma-like reaction.

The inducible knockout $\mathrm{GR}^{\text {SPCcreERT2 }}$ mice were generated by crossing the $\mathrm{GR}^{\text {flox/flox }}$ with

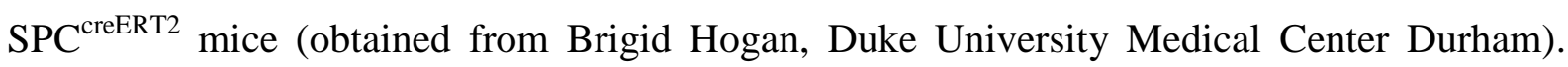
This mouse express a tamoxifen-inducible Cre recombinase (Cre-ERT2) (Feil et al., 1997;Rock et al., 2011; Xu et al., 2012), which is under the control of the surfactant protein C promoter (SPC). Because the SPC promoter is specific for alveolar type II epithelial cells, the $\mathrm{GR}^{\mathrm{SPC} C r e E R T 2}$ mice are lacking the GR expression only in these lung epithelial cells. The $\mathrm{GR}^{\mathrm{SPC} C r e E R T 2}$ mice were backcrossed to $\mathrm{C} 57 \mathrm{Bl} / 6$ and used to analyze the role of alveolar epithelial cells as the possible key player in the treatment of asthma-like reaction with GCs.

\subsubsection{Technical equipment}

\begin{tabular}{l|l} 
Centrifuge & Manufacturer \\
\hline Centrifuge Sigma 2-5 for 96-well plates & $\begin{array}{l}\text { Sigma Laborzentrifugen GmbH, } \\
\text { Osterode am Harz, Germany } \\
\text { Eppendorf, Hamburg, Germany }\end{array}$ \\
Centrifuge 5417R for reaction tubes & Eppendorf, Hamburg, Germany \\
Centrifuge 5804R for FACS tubes & Heraeus, Hanau, Germany \\
Centrifuge Multifuge 4 KR for Falcon tubes &
\end{tabular}

\begin{tabular}{l|l} 
Microscope & Manufacturer \\
\hline Microscope Primo Star & Zeiss, Jena, Germany \\
Microscope Telavel 31 & Zeiss, Jena, Germany
\end{tabular}




\begin{tabular}{l|l}
\hline Incubator & Manufacturer \\
\hline Incubator, HERAcell 240 & Heraeus, Hanau, Germany \\
Incubator HERAcell150 & Hereaus, Hanau, Germany \\
Incubator HERAeus Functionline & Hereaus, Hanau, Germany
\end{tabular}

\begin{tabular}{l|l} 
Bench & Manufacturer \\
\hline Laminar airflow cabinet, HERASafe & Heraeus, Hanau, Germany \\
The Baker company, Sanford, Maine, \\
Laminar airflow cabinet Steril Gard Hood & USA \\
Interactive SafeChange station (animal facility) & $\begin{array}{l}\text { TECNIPLAST Deutschland GmbH, } \\
\text { Hohenpeißenberg, Germany }\end{array}$
\end{tabular}

\begin{tabular}{l|l} 
Water bath & Manufacturer \\
\hline Water bath W12 & $\begin{array}{l}\text { Labortechnik Medigen, Dresden, } \\
\text { Germany }\end{array}$ \\
Water bath Medax & Nagel GmbH, Kiel, Germany
\end{tabular}

\begin{tabular}{l|l} 
Thermocycler & Manufacturer \\
\hline Thermocycler & Heraeus, Hanau, Germany \\
7500 Real-Time PCR System & $\begin{array}{l}\text { AB Applied System Applera GmbH, } \\
\text { Darmstadt, Germany } \\
\text { Thermomixer Comfort }\end{array}$ \\
Eppendorf, Hamburg, Germany
\end{tabular}




\begin{tabular}{l|l}
\hline Photometer & Manufacturer \\
\hline $\begin{array}{l}\text { Nanodrop ND-1000 Spectrophotometer } \\
\text { Photometer }\end{array}$ & ThermoScientific, Wilmington, USA \\
BioTek® Power Wave 340 plate reader & Eionendorf, Hamburg, Germany \\
&
\end{tabular}

\begin{tabular}{l|l} 
Refrigerator & Manufacturer \\
\hline Freezer Liebherr Comfort $-20^{\circ} \mathrm{C}$ & $\begin{array}{l}\text { Liebherr International Deutschland } \\
\text { GmbH, Biberach an der Riss, Germany } \\
\text { Heraeus, Hanau, Germany }\end{array}$ \\
Freezer Hera $-80^{\circ} \mathrm{C}$ & $\begin{array}{l}\text { Sanyo Electric Co. Moriguchi, Osaka, } \\
\text { Japan }\end{array}$ \\
Freezer VIP plus $-150^{\circ} \mathrm{C}$ &
\end{tabular}

\begin{tabular}{l|l} 
FACS device & Manufacturer \\
\hline BD FacsCanto II & Becton Dickinson Biosciences, \\
Heidelberg, Germany \\
$\begin{array}{l}\text { BD FACS Sorter (BD FACSAria special order } \\
\text { research product) }\end{array}$ & BD Bioscience, Heidelberg, Germany
\end{tabular}

\begin{tabular}{l|l} 
Other used devices & Manufacturer \\
\hline Vortex Genie-2 & $\begin{array}{l}\text { Bender\&Hobein AG, Zürich, Swiss } \\
\text { Gulmay Medical Systems, Camberley, } \\
\text { Surrey, UK } \\
\text { RS 225 X-Ray Research System }\end{array}$ \\
Vapöger Medical Gmbh, Lübeck, \\
Germany \\
T18 basic Ultra-Turrax®high performance & Uni Göttingen, Germany \\
dispenser & IKA, Staufen, Germany
\end{tabular}


Neubauer improved haemacytometer

UV System camera and gel imager

ChemoStar gel imager,

Accu-jet ${ }^{\circledR}$ pro pipette controller

Scale 1003

Heating plate HI1220

Microtom Leica SM2000R

Dehydrationssystem TP1020

Tissue embedding system EG1160

Microplatte Counter 2450 Microbeta $^{2}$

Filtermate Harvester

Arium®611 laboratory water purification system

Millipore Purification System
Henneberg-Sander GmbH, Gießen, Germany

Intas $\mathrm{GmbH}$, Göttingen, Germany

Intas GmbH, Göttingen, Germany

Brand GmbH, Wertheim, Germany

Sartorius, Göttingen, Germany

Leica Microsystems, Nussloch, Germany

Leica Microsystems, Nussloch, Germany

Leica Microsystems, Nussloch, Germany

Leica Microsystems, Nussloch, Germany

Perkin Elmer precisely, Downers Grove, USA

Perkin Elmer, Downers Grove, USA

Sartorius AG, Göttingen, Germany

Millipore GmbH Schwalbach, Germany

\subsubsection{Consumables}

\section{Consumables \\ Cannula BD Microlance 3 20G 1/2}

$(0.9 \times 40 \mathrm{~mm})$

Cannula B. Braun Sterican 26G 1/2

$(0.45 \times 12 \mathrm{~mm})$

Venous catheter BD Venflon Pro 22GA

$(0.9 \times 25 \mathrm{~mm})$

Syringe BD Discardit II (10 and $20 \mathrm{ml}$ )

Syringe BD Plastipak $1 \mathrm{ml}$

\section{Manufacturer}

BD Biosciences, Heidelberg, Germany

B. Braun, Melsungen. Germany

BD Biosciences, Heidelberg, Germany

BD Biosciences, Heidelberg, Germany

BD Biosciences, Heidelberg, Germany 
Diabetic syringe Omnifix $1 \mathrm{ml}$ (25G x 5/8”)

FACS tubes

ELISA Plates

Centrifuge tubes (15 and $50 \mathrm{ml})$

Round bottom culture tube (14 ml)

PCR tubes

Disposable pipettes $(5 \mathrm{ml}, 10 \mathrm{ml}$ and $25 \mathrm{ml}$ )

Glas pipettes $(5 \mathrm{ml}$ and $10 \mathrm{ml})$

Pipette tips for volume $100 \mu \mathrm{l}-1000 \mu \mathrm{l}$

Pipette tips for volume $10 \mu 1-200 \mu 1$

Pipette tips for volume $0.1 \mu \mathrm{l}-10 \mu \mathrm{l}$

24-/ 48-well suspension culture plates

96-well optical reaction plate MicroAmp

Optical adhesive covers
B. Braun, Melsungen AG, Germany

Greiner Bio-One, Frickenhausen, Germany

NUNC, Roskilde, Denmark

Greiner Bio-One, Frickenhausen, Germany

Greiner Bio-One, Frickenhausen, Germany

Sarstedt, Nümbrecht, Germany

Greiner Bio-One, Frickenhausen, Germany

Brand Gmbh+CO KG, Hamburg, Germany

Sarstedt, Nümbrecht, Germany

Greiner Bio-One, Frickenhausen, Germany

Sarstedt, Nümbrecht, Germany

Greiner Bio-One, Frickenhausen,

Germany

AB Applied BioSystems, Foster City, USA

AB Applied BioSystems, Foster City, USA

\subsubsection{Chemicals}

\begin{tabular}{l|l} 
Chemicals & Manufacturer \\
\hline FCS & Gibco, Invitrogen, Karlsruhe, Germany \\
BSA & Roth, Karlsruhe, Germany \\
DEPC & Sigma-Aldrich, Taufkirchen, Germany
\end{tabular}




\section{DNase I}

Ethanol

Glucose

Isopropanol

$\mathrm{H}_{2} \mathrm{O}_{2} 30 \%$

$\mathrm{H}_{2} \mathrm{SO}_{4}$

$\mathrm{HCl}$

HEPES

ß-Mercaptoethanol

$\mathrm{KCl}$

$\mathrm{KH}_{2} \mathrm{PO}_{4}$

$\mathrm{KHCO}_{3}$

$\mathrm{Na}_{2} \mathrm{CO}_{3}$

$\mathrm{Na}_{2} \mathrm{HPO}_{4} \times 12 \mathrm{H}_{2} \mathrm{O}$

$\mathrm{Na}_{3} \mathrm{C}_{6} \mathrm{H}_{5} \mathrm{O}_{7}$

$\mathrm{NaCl}$

$\mathrm{NaH}_{2} \mathrm{PO}_{4} \times \mathrm{H}_{2} \mathrm{O}$

$\mathrm{NaHCO}_{3} \times 3 \mathrm{H}_{2} \mathrm{O}$

$\mathrm{NaN}_{3}$

$\mathrm{NaOH}$

Neomycin trisulfate salt hydrate

$\mathrm{NH}_{4} \mathrm{Cl}$

Proteinase K

Taq-Polymerase $(5 \mathrm{U} / \mu \mathrm{l})$

Tris
Roche, Basel, Swiss

Roth, Karlsruhe, Germany

Ferak, Berlin, Germany

Roth, Karlsruhe, Germany

Carl Roth, Karlsruhe, Germany

Merck, Darmstadt, Germany

Carl Roth, Karlsruhe, Germany

Merck, Darmstadt, Germany

Roth, Karlsruhe, Germany

Merck, Darmstadt, Germany

Merck, Darmstadt, Germany

Merck, Darmstadt, Germany

Merck, Darmstadt, Germany

Merck, Darmstadt, Germany

Carl Roth, Karlsruhe, Germany

Carl Roth, Karlsruhe, Germany

Merck, Darmstadt, Germany

Merck, Darmstadt, Germany

Carl Roth, Karlsruhe, Germany

SIGMA-Aldrich, Taufkirchen, Germany

SIGMA-Aldrich, Taufkirchen, Germany

Merck, Darmstadt, Germany

AppliChem, Darmstadt, Germany

Genaxxon Bioscience GmbH, Germany

AppliChem, Darmstadt, Germany 
Trizol

Penicillin/Streptomycin

Tween 20

3,3',5,5'-Tetramethylbenzidine

Agarose UltraPure

Dexa-ratiopharm® 100 mg Injektionslösung

DMSO

Ethidium bromide

EDTA

$\mathrm{CaCl}_{2} \times 2 \mathrm{H}_{2} \mathrm{O}$

Citric acid

Sevorane Sevoflurane

Paraformaldehyde (PFA)

Protease inhibitor cocktail

PfuS DNA polymerase

Alhydrogel 2\% (Alum)

Albumin from chicken egg white (Ovalbumine/ OVA)

Concanavalin A

DNA ladder $1 \mathrm{~kb}$
Invitrogen, Karlsruhe, Germany

Gibco, Invitrogen, Karlsruhe, Germany

Carl Roth Gmbh, Karlsruhe, Germany

SIGMA-Aldrich, Taufkirchen, Germany

Invitrogen, Paisley, UK

Ratiopharm GmbH, Ulm, Germany

Carl Roth Gmbh, Karlsruhe, Germany

Carl Roth Gmbh, Karlsruhe, Germany

Serva, Heridelberg, Germany

Merck, Darmstadt, Germany

Merck, Darmstadt, Germany

Abbott, Illinois, USA

SIGMA-Aldrich, Taufkirchen, Germany

SIGMA-Aldrich, Taufkirchen, Germany

Own production

InvivoGen, Toulouse, France

SIGMA-Aldrich, Taufkirchen, Germany

Sigma-Aldrich, Taufkirchen, Germany

Fermentas GmbH, St. Leon-Rot,

Germany

\subsubsection{Oligonucleotide}

\begin{tabular}{l|l} 
Oligonucleotide & Sequenz \\
\hline$\beta$-Tryptase & $\begin{array}{l}\text { 5'AGG CAG CTA AGA TGC TGA AGC GG-3' }^{\prime} \text { '-CTC CCA CGA TGC CCA CTC GC-3' }\end{array}$
\end{tabular}


CCL11 (Eotaxin-1)

CCL24 (Eotaxin-2)

CXCR3

CXCR4

CXCL12 (SDF-1)

GILZ

HPRT

iNOS

IL-4

IL-5

IL-9

IL-10

IL-13

IL-17

IL-25

IL-33

MIP-1a (CCL3)

MCP-1 (CCL2)

ZO-1
5 -CGG GAG AGC CTA CAG AGC CCA G-3`

5 -GGG ATG GAG CCT GGG TGA GCC-3`

5 -TCC CAA GGC AGG GGT CAT CTT CA-3`

5 -TCG GTG CTA TTG CCA CGG CG-3

5 `ACT GCT CTG CGT GTA CTG CA-3`

5-CCA CTA CCA CTA GCC TCA TAG-3

5'-GGC GTC TGG ATC CCA GCC CT-3'

5'-GGC AGG ACG AGA CCC ACC A-3'

5'-CCC TTC AGA TTG TTG CAC GG-3'

5'-TCC TTT GGG CTG TTG TGC TT-3'

5'-TTC CGG GGT TTC CGG GGT TTC-3'

5'-GTG AGA GAG GAG GTG GAG-3'

5'-GTC CTG TGG CCA TCT GCC TA-3'

5'-GGG ACG CAG CAA CTG ACA TT-3`

5'-CCG CAC CCG AGA TGG TCA GG-3'

5'-GCA AGG CTG GGA GGG GTC CT-3'

5'-CCC CCA GCT AGT TGT CAT CCT GC-3'

5'-ATC TCC CTG CAT GGC GTC CCT-3'

5'-TGC TGA AGG CCAGCG CTG AAG-3`

5'-GGG ACA GGA AGC CTC ATC GTC TCA T-3`

5'-GGC CAG AGA TGC AGC ACC A-3'

5'-AGC AGC TGG TCA CGT TGC CG-3'

5'-AGG CAG AGA AGC ATG GCC CAG A-3'

5'-CGG GAG AAA TCG ATG ACA GCG CC-3'

5-CCC CTG TGC AAC GGC AGC AT-3`

5 -CGG GGA GGC TGG AGA CCG TA-3`

5-AAA GCT CAG CGT GTC CAA AC-3

5-CTT CAT TGC GGT GGA GAG TC-3`

5'-TGT CCG GCA TGT ACC AGG CTG T-3'

5'-GCC ACT CCT CCG GGG GTT CT-3'

5'-TCA ATC AGG CGA CGG TGT GGA-3'

5'-AAG GCC TGT TCC GGA GGC GA-3'

5'-CAG CCA GGT GTC ATT TTC CT -3'

5'-CTC AAG CCC CTG CTC TAC AC-3'

5'-AGC ACC AGC CAA CTC TCA CT-3'

5'-CGT TAA CTG CAT CTG GCT GA-3'

5'-CCA GCC TCT CAA CAG GTG TA-3'

5'-TCA AGG TCT CTG CTG GCT TG-3' 
Occludin

RANTES (CCL5)

TNF- $\alpha$

TSLP

TGF- $\beta$

GRquant

GT3’GT5’
5'-CCT CCA CCC CCA TCT GAC TA -3'
5'-CTT CAG GCA CCA GAG GTG TT -3'
5'-GTG CCC ACG TCA AGG AGT AT-3'
5'-GGA AGC GTA TAC AGG GTC A-3'
5-CGT CAG CCG ATT TGC TAT CT-3`
5 -CGG ACT CCG CAA AGT CTA AG-3`
5'-AGG ACT GTG AGA GCA AGC CAG C-3'
5'-AGG GTA GCC TGG GCA GTG GT-3'
5'-TGA CGT CAC TGG AGT TGT ACG G-3'
5'-GGT TCA TGT CAT GGA TGG TGC-3'
5'-CAG CAA CGG GAC CAC CTC CC-3'
5'-GTG CTG TCC TTC CAC TGC TCT-3'
5'-CCA TTA CCT TCC AGG TTC ATT C-3'
5'-GTG TCT TGA TGA TAG TCT GCT C-3'

All oligonucleotides were designed using the Primer-BLAST software and purchased from Metabion (Martinsried, Germany).

\subsubsection{Media and Buffers}

\begin{tabular}{|c|c|}
\hline Media and Buffers & Components or Manufacturer \\
\hline PBS & $\begin{array}{l}13 \mathrm{mM} \mathrm{NaCl} \\
2.6 \mathrm{mM} \mathrm{KCl} \\
6.5 \mathrm{mM} \mathrm{Na}_{2} \mathrm{HPO}_{4} * 2 \mathrm{H}_{2} \mathrm{O} \\
3.6 \mathrm{mM} \mathrm{CaCl}_{2} * 4 \mathrm{H}_{2} \mathrm{O} \\
0.5 \mathrm{mM} \mathrm{MgCl}_{2} * 6 \mathrm{H}_{2} \mathrm{O}\end{array}$ \\
\hline $\mathrm{PBS} / \mathrm{BSA}$ & $0.1 \% \mathrm{BSA}$ in $\mathrm{PBS}$ \\
\hline FACS Buffer & $\begin{array}{l}0.1 \% \mathrm{BSA} \text { in PBS, pH } 7.2 \\
0.01 \% \mathrm{NaN}_{3}\end{array}$ \\
\hline RPMI 1640 & Gibco, Invitrogen, Karlsruhe, Germany \\
\hline RPMI-10 & $\begin{array}{l}\text { RPMI } 1640 \\
10 \% \text { heatinactivated FCS }\left(30 \text { minutes at } 56{ }^{\circ} \mathrm{C}\right) \\
1 \% \text { Penicillin/Streptomycin }\end{array}$ \\
\hline TAC Buffer & $20.0 \mathrm{mM}$ Tris/HCl pH 7.2 \\
\hline
\end{tabular}




\begin{tabular}{|c|c|}
\hline & $155 \mathrm{mM}$ Ammonium chloride \\
\hline DMEM & Gibco, Invitrogen, Karlsruhe, Germany \\
\hline ELISA Assay diluents & $10 \%$ FCS in PBS \\
\hline ELISA Coating Buffer & 0.1 M Sodium carbonate, $\mathrm{pH} 9.5$ \\
\hline ELISA Wash Buffer & $0.05 \%$ Tween-20 in PBS \\
\hline ELISA Substrate Buffer & $\begin{array}{l}\text { 0.1M Citric Acid } \\
0.2 \mathrm{M} \mathrm{Na}_{2} \mathrm{HPO}_{4} \times 2 \mathrm{H}_{2} \mathrm{O} \\
\mathrm{dH}_{2} \mathrm{O}\end{array}$ \\
\hline ELISA developing solution & $\begin{array}{l}1 \% \mathrm{TMB} \text { in DMSO }(0.01 \mathrm{~g} / \mathrm{ml}(\mathrm{w} / \mathrm{v})) \\
0.2 \% \mathrm{H}_{2} \mathrm{O}_{2}(3.5 \% \text { solution }) \\
\text { Substrate buffer }\end{array}$ \\
\hline ELISA Stop Solution & $2 \mathrm{~N} \mathrm{H}_{2} \mathrm{SO}_{4}$ \\
\hline Alsevers & $\begin{array}{l}27 \mathrm{mM} \mathrm{NaCl} \\
125 \mathrm{mM} \text { D-Glucose } \\
3 \mathrm{mM} \text { Citric acid } \\
30 \mathrm{mM} \mathrm{Na}_{3} \mathrm{C}_{6} \mathrm{H}_{5} \mathrm{O}_{7}\end{array}$ \\
\hline $10 \times$ DNaseI-Buffer & $\begin{array}{l}500 \mathrm{mM} \text { Tris-HCl, } \mathrm{pH} 7.5 \\
50 \mathrm{mM} \mathrm{CaCl}_{2} \\
10 \mathrm{mM} \text { DTT }\end{array}$ \\
\hline 50x TAE Buffer & $\begin{array}{l}4.8 \mathrm{~g} \text { Tris } \\
1.1 \mathrm{~g} \text { glacial } \mathrm{C}_{2} \mathrm{H}_{4} \mathrm{O}_{2} \\
0.29 \mathrm{~g} \text { EDTA } \\
1 \mathrm{~L} \mathrm{dH}_{2} \mathrm{O}\end{array}$ \\
\hline Tail-extraction Buffer & $\begin{array}{l}5 \mathrm{mM} \text { Tris/HCl (pH 8.0) } \\
100 \mathrm{mM} \mathrm{NaCl} \\
100 \mathrm{~mm} \text { EDTA (pH 8.0) } \\
1 \% \mathrm{SDS}\end{array}$ \\
\hline
\end{tabular}




\subsubsection{Antibodies}

\begin{tabular}{l|lll} 
Antigen & Fluorochrome & Isotype & Clone \\
\hline CD4 & APC & Rat IgG2a, $\kappa$ & RM4-5 \\
CD3 & PerCP & Rat IgG2b, $\kappa$ & 17 A2 \\
F4/80 & FITC & Rat IgG2b, $\kappa$ & CI:13-1 \\
CD11b & PE-Cy7 & Rat IgG2b, $\kappa$ & M1/70 \\
Gr-1 (Ly-6G/C) & APC-Cy7 & Rat IgG2b, $\kappa$ & RB6-8C5 \\
Siglec-F & PE & Rat IgG2a, $\kappa$ & E50-2440 \\
CD16/CD32 & purified & Ms IgG1, $\kappa$ & $2.4 \mathrm{G} 2$ \\
Podoplanin & PE & Syrian Hamster IgG & 8.11 \\
CD45.2 & APC & Ms (SJL) IgG2a, $\kappa$ & 104 \\
CD34 & PeCy5 & Rat IgG2a $\kappa$ & MEC 14.7
\end{tabular}

The Antibodies for flow cytometry were purchased from BD Biosciences (Heidelberg, Germany) or Biolegend (San Diego, USA).

\subsubsection{Commercial assays}

\begin{tabular}{|c|c|}
\hline Kits & Manufacturer \\
\hline Qiagen RNeasy® Plus Universal Kit & Qiagen, Hilden, Germany \\
\hline iScript cDNA Synthesis Kit & Bio-Rad, Hercules, CA, USA \\
\hline Power SYBR $®$ green & $\begin{array}{l}\text { Applied Biosystems, Foster City, California, } \\
\text { USA }\end{array}$ \\
\hline $\begin{array}{l}\text { dNTPs (dATP } \mathrm{Na}_{4} \text { × } 3 \mathrm{H}_{2} \mathrm{O} \text {, dCTP } \mathrm{Na}_{4} \text { × } 3 \\
\mathrm{H}_{2} \mathrm{O} \text {, dGTP } \mathrm{Na}_{4} \text { × } 3 \mathrm{H}_{2} \mathrm{O} \text {, dTTP } \mathrm{Na}_{4} \text { × } 3 \mathrm{H}_{2} \mathrm{O} \text { ) }\end{array}$ & Genaxxon bioscience, Ulm, Germany \\
\hline $\begin{array}{l}\text { 5x Phusion }{ }^{\circledR} \text { Reaction Buffer HF with } \\
7.5 \mathrm{mM} \mathrm{MgCl}_{2}\end{array}$ & $\begin{array}{l}\text { Thermo Scientific, Waltham, Massachusetts, } \\
\text { USA }\end{array}$ \\
\hline $\begin{array}{l}\text { IgE-HRP (goat anti-mouse) } \\
\text { IgG2a-HRP (goat anti-mouse) }\end{array}$ & Southern Biotech, Birmingham, USA \\
\hline
\end{tabular}




\begin{tabular}{l|l}
\hline IgG1-HRP (goat anti-mouse) & \\
\hline Mouse IL-2 ELISA MAX ${ }^{\mathrm{TM}}$ Standard Set & \\
Mouse IL-4 ELISA MAX ${ }^{\mathrm{TM}}$ Standard Set & Biolegend, San Diego, USA \\
Mouse IL-5 ELISA MAX ${ }^{\mathrm{TM}}$ Standard Set \\
Mouse IFN $\gamma$ ELISA MAX ${ }^{\mathrm{TM}}$ Standard Set & \\
\hline BD OptEIA ${ }^{\mathrm{TM}}$ Set Mouse IL-2 & BD Bioscience, Heidelberg, Germany \\
BD OptEIA ${ }^{\mathrm{TM}}$ Set Mouse IL-4 & \\
BD OptEIA ${ }^{\mathrm{TM}}$ Set Mouse IFN $\gamma$ & Zymo Research, Freiburg Germany \\
\hline Mini RNA Isolation Kit & Qiagen, Hilden, Germany \\
\hline RNease Mini Kit &
\end{tabular}

\subsubsection{Software}

\begin{tabular}{l|l} 
Software & Source/Manufacturer \\
\hline BioTek ${ }^{\circledR}$ Gen5 ${ }^{\mathrm{TM}}$ version 1.09 & BioTek, Bad Friedrichshall, Germany \\
7500 System SDS Software v. 1.4.0.25 & $\begin{array}{l}\text { Applied Biosystems, Foster City, California, } \\
\text { USA } \\
\text { BD Biosciences, Heidelberg, Germany }\end{array}$ \\
BD FACS Diva software version 6.1.2 & $\begin{array}{l}\text { Tree Star, Inc., Ashland, Oregon, USA } \\
\text { GraphPad Software, La Jolla, CA, USA }\end{array}$ \\
FlowJo software version 7.6 & $\begin{array}{l}\underline{\text { http://www.ncbi.nlm.nih.gov/tools/primer- }} \\
\text { blast/ } \\
\text { Prism version 6.0 }\end{array}$ \\
Primer-BLAST & $\begin{array}{l}\text { ART Advanced Research Technologies Inc., } \\
\text { Montreal, Canada }\end{array}$ \\
OptiView-2 02-00 software (ART) &
\end{tabular}




\subsection{Methods}

\subsubsection{Animal work}

\subsubsection{Induction of experimental asthma}

In order to investigate mechanisms of GCs in the treatment of asthma in a murine model, it is a prerequisite to induce robust allergic airway inflammation (AAI). This can be achieved by immunizing and challenging mice with specific antigens (Zosky et al., 2007; Campbell et al., 1999; Clarke et al., 1999; Tounoy et al., 2000) in combination with a potent adjuvant such as aluminium hydroxide (Alum).

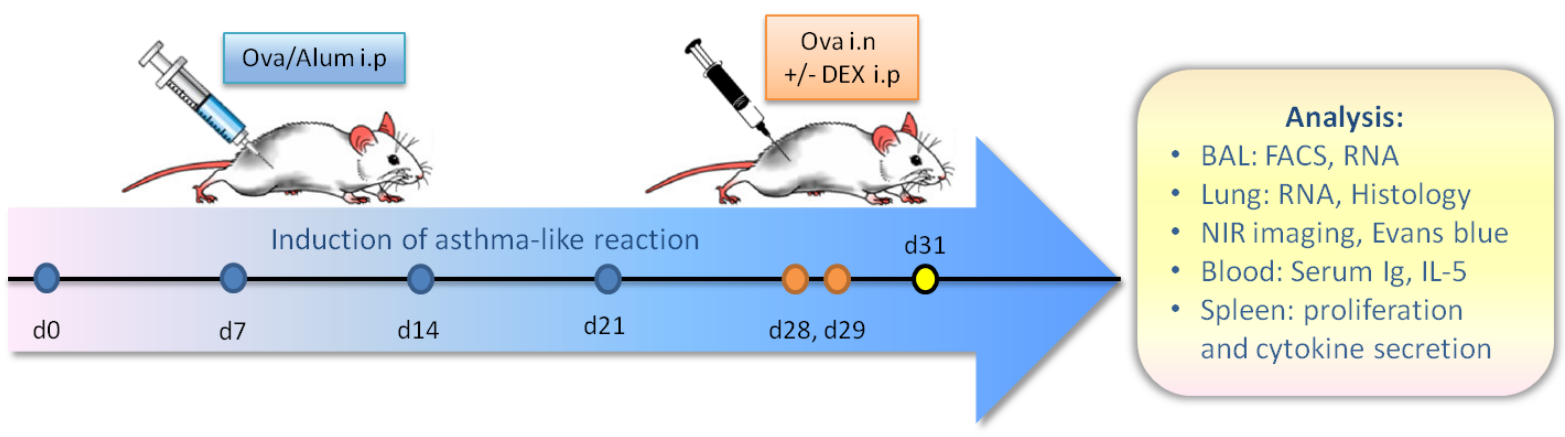

Figure 10: AAI induction schema. For the induction of experimental asthma, mice were immunized by intraperitoneal injection once a week for 4 consecutive weeks with Ovalbumin (Ova) emulsified in aluminium hydroxide (Alum) or with Alum in PBS alone (control group). Four weeks after immunization, the mice were challenged with intranasal applications of Ova. One group of Ovaimmunized and challenged mice remained untreated whereas another group was treated with Dexamethasone (Dex) intraperitoneally. About 40 hours after the last challenge samples were collected.

Immunization with chicken-egg Ovalbumin (Ova) as the allergen induces a robust, allergic pulmonary inflammation in laboratory rodents (Kumar et al., 2008) and is a widely used model to induce airway eosinophilia, pulmonary inflammation and elevated $\operatorname{IgE}$ levels found during asthma in patients. To induce AAI in the lungs, 8-12 week-old mice were immunized on day $0,7,14$ and day 21 by intraperitoneal injections of $10 \mu \mathrm{g}$ of Ova diluted in sterile PBS and emulsified in $2 \mathrm{mg}$ Alum. The Ova was allowed to conjugate to the Alum for $1 \mathrm{hr}$ at the room temperature on the shaker before immunization. Control mice received intraperitoneal injections of Alum alone. On day 28 and 29, the mice intranasally received $250 \mu \mathrm{g}$ Ova solubilised in sterile PBS with or without GC-treatment via intraperitoneal injection of Dexamethasone (10 mg per $1 \mathrm{~kg}$ body weight) 1 hour after each Ova-challenge. On day 31, about 40 hours after the last challenge, the bronchoalveolar lavage (BAL) fluid 
was collected from the lungs. In addition, the spleen, blood and the lung tissue was collected to study inflammation using FACS, ELISA, RT-qPCR and histological analyses.

\subsubsection{Establishing of bone marrow chimeras}

To investigate how important GR-expression on non-immune cells is for the therapeutic efficiency of GCs in the treatment of asthmatic reactions, bone marrow chimeras with $\mathrm{GR}^{\mathrm{wt}}$ or $\mathrm{GR}^{\mathrm{dim}}$ mice as recipients and $\mathrm{GR}^{\mathrm{wt}}$ mice or $\mathrm{GR}^{\mathrm{dim}}$ mice as donors of bone marrow cells were generated.

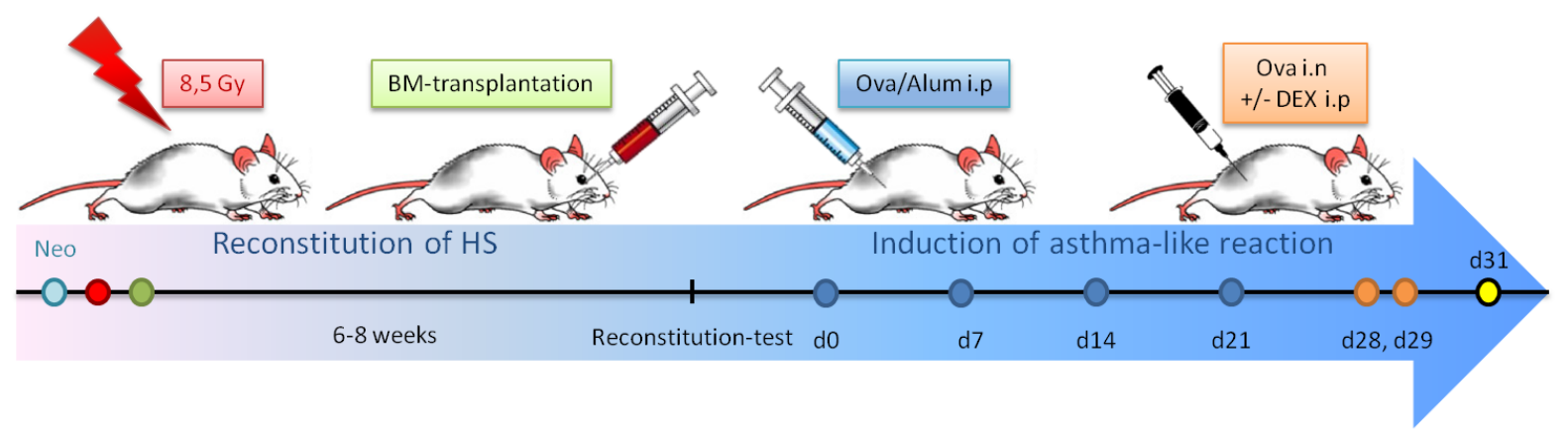

Figure 11: Reconstitution schema of bone marrow chimeras. The reconstitution of the hematopoietic system was achieved via transfer of bone marrow into irradiated recipient mice. About 6-8 weeks after the transplantation and successfully reconstitution of the hematopoietic system the AAI was induced in the chimeric animals.

To reconstitute the host's hematopoietic system by donor cells, recipient mice at the age of 8-10 weeks were lethally irradiated with 8.5 Gy at a rate of 1 Gy per minute using a RS 225 X-Ray Research System (Gulmay Medical Systems) operated at 200 kV, 15 mA and with $0.5 \mathrm{~mm} \mathrm{Cu}$ filtration to delete their own hematopoietic cells.

To prevent infection of the irradiated mice $25 \mu \mathrm{g} / \mathrm{ml}$ Neomycin were added to the drinking water starting one day before irradiation. The water with Neomycin was changed every third day during the following six weeks.

On the next day animals received bone marrow cells from donor mice. To isolate bone marrow cells from the donor mice, they were euthanized with $\mathrm{CO}_{2}$. After removing the skin from the legs, the muscle was cut safety from the femur and tibia. The bones were isolated and placed in PBS on ice. After that, the tibia was separated from the femur at the knee level. The ends of the bones were cut off under sterile conditions. Through the small hole in the end of the bone the bone marrow was flushed out using a 26-gauge needle placed on the 
$5 \mathrm{ml}$ syringe filled with ice cold PBS containing $0.1 \%$ BSA. The isolated bone marrow was resuspended using a 30-gauge needle. The resulting cell suspension was passed through a $40 \mu \mathrm{M}$ cell strainer to remove large fragments and washed in PBS by centrifugation for 5 minutes at $350 \mathrm{~g}$ and $4{ }^{\circ} \mathrm{C}$. Afterwards the cell number was determinate and the bone marrow cell suspension was diluted with PBS to achieve a final concentration of $4 \times 10^{7}$ cells per $100 \mu$ l. The resulting cell suspension was stored on ice until intravenous injection into recipient mice.

\subsubsection{Induction of epithelial cell-specific knockout}

To analyse the role of alveolar epithelial cells as possible key players in the treatment of asthma-like reactions with GCs an inducible $\mathrm{GR}^{\text {SPCcreERT2 }}$ mice were used.

To study the function of lung epithelial cells a SPC ${ }^{\text {creERT2 }}$ mouse was generated by Brigid Hogan from Duke University Medical Center Durham. This mouse expresses a tamoxifeninducible Cre recombinase $\left(\mathrm{Cre}-\mathrm{ER}^{\mathrm{T} 2}\right)$, which is under the control of the surfactant protein $\mathrm{C}$ (SPC) promoter. To this end, the Cre coding sequence was fused to a mutated ligandbinding domain of the estrogen receptor $\left(\mathrm{ER}^{\mathrm{T} 2}\right)$. This mutated ligand-binding domain retains the fusion protein in the cytoplasm and can translocate into the nucleus (with following excision of the loxP-flanked DNA sequence) only upon binding of tamoxifen as a ligand (Kellendonk et al., 1996; Feil et al., 1997). Because the surfactant protein C is exclusively expressed in the type II alveolar epithelial cells, this knockout occurs only in lung epithelia. In this study the SPC ${ }^{\text {creERT2 }}$ mice, kindly provided by Brigid Hogan were crossed with GR $^{\text {flox }}$ mice to obtain alveolar epithelia specific knockout of the GR in GR ${ }^{\text {SPCcreERT2 mice. }}$ Expression of the GR in alveolar epithelial cells was evaluated using RT-qPCR analysis of isolated epithelial cells.

\section{Tamoxifen treatment of mice}

An aliquot of tamoxifen was dissolved in sun flower oil. This $20 \mathrm{mg} / \mathrm{ml}$ tamoxifen solution was dissolved by incubation at $56{ }^{\circ} \mathrm{C}$ for about $20 \mathrm{hrs}$ in a water bath with repeated mixing on a vortexer. For oral administration the solution was administered to the mice three times ( $3 \mathrm{mg}$ of tamoxifen dissolved in $150 \mu \mathrm{l}$ ) by a feeding needle every other day. Then, 2 days after the last tamoxifen administration the mice were used for induction of allergic asthmalike reaction in the lungs. 


\subsubsection{Histological and imaging analysis of the lung}

\subsection{NIRF Imaging}

In vivo imaging by Fluorescence Molecular Tomography (FMT) and Near-Infrared Fluorescent (NIRF) Imaging is relatively new method to investigate biological processes in mouse models of diseases. In the last few years the new Optical Imaging devices (like the FMT 2500 device applied in this work) were developed and now are available for animal applications. The feature of this technique is to provide an insight into the cellular processes in the body (during for example an inflammatory disease) via a non-invasive mode of action in the real time. Disease progression and therapeutic responses, cells of interest or biological markers can be monitored and quantified through labelling with light emitting reporters (like ProSense680 or anti-SiglecF-AlexaFluor750) (Lyons SK, 2005; Pomper et al, 2005; Hickson J et al, 2009; Leblond F et al, 2010).

An imaging agent applied in this work for the examination of asthma-like reactions in the lungs of Ovalbumin-sensitised mice was ProSense680, a near-infrared (NIR) proteasesensing imaging agent. Because this dye is only protease-activatable, it is optically silent in its unactivated state in the absence of proteases such Cathepsin B, L, S and Plasmin. These Cathepsins are expressed at basal levels in all living cells (Turk et al, 2001), they play an important role in several physiological processes such as lysosomal protein degradation, antigen presentation and apoptosis. The application of ProSense680 for the monitoring of inflammatory diseases is depended on the specific characteristic of the cathepsins, namely these proteases are associated with pathological conditions and diseases such as cancer, osteoporosis and bronchial asthma (Turk et al 2001; Zavasnik-Bergant \& Turk, 2006; Vasiljeva et al, 2007). In addition, cathepsins are active in intracellular lysosomes of inflammatory eosinophils, which leads to the accumulation of the imaging agent in the lungs in vivo.

As another marker for inflammation in the lungs an AlexaFluor750 labeled anti-SiglecF antibody was used. In mouse, Siglec-F is an inhibitory receptor specifically expressed on eosinophils. Because one of the typical characteristic of asthmatic reaction is a strong eosinophilia in the lungs, the anti-SiglecF-AlexaFluor750 provides the opportunity to analyse the eosinophil influx into the lungs, the severity of asthmatic reactions and effects of GC-therapy on eosinophil migration. 


\subsection{In vivo imaging procedure and analysis}

After the second Ovalbumin-challenge the mice were narcotized using a $4 \%$ Isofluran/Oxygen mix produced in a Dräger Vapor 2000 device and transferred into a customized Perspex chamber. Afterwards, the mice were depilated to minimize fur interference with the fluorescent signal. Depilating crème was applied thickly onto the skin over the upper torso (front and both sides) of each mouse, after a few minutes the crème was rinsed off with warm water; this procedure was repeated until all fur was removed from the regions of interest. $4 \mathrm{hrs}$ later, the control mice and the Ovalbumin-sensitized mice were imaged using the FMT 2500 fluorescence tomography in vivo imaging system (VisEn Medical, Inc.) to determine the basal fluorescence from the lungs and from other body regions before injection of the fluorescence imaging agent. After this prescan the mice were

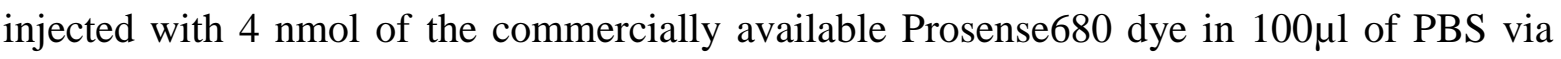
intravenous administration or $12 \mu \mathrm{g}$ anti-SiglecF-AlexaFluor750 diluted in $100 \mu \mathrm{l}$ PBS.

After 24 hours the mice were narcotized and carefully positioned in the imaging chamber in the FMT device. With the help of a NIR laser diode the light was passed through the mouse body and then collected on the opposite side by signal detection via a thermoelectrically cooled charge-coupled device camera. The image acquisition took approximately 4 to 5 min per mouse. Scans were performed with $1.5 \mathrm{~mm}$ (whole body scans) or $1.0 \mathrm{~mm}$ (regions of interest) raster, photon collection time (so called integration time) of $0.5-0.7$ s per scan point and varying laser power. Prosense680 fluorescence was measured using excitation at 670 $\mathrm{nm}$ in combination with a $700 \mathrm{~nm}$ long-pass emission filter. The fluorescence of antiSiglecF-AlexaFluor750 was analysed using excitation at $730 \mathrm{~nm}$. The collected fluorescence data were analysed with the OPTIX-OptiView-2 02-00 (ART) software. The obtained fluorescence signal was normalized to the basal fluorescence of each animal (prescan) and the laser power which was used during acquisition. The relative fluorescence unit intensity and the distribution in lung tissues were automatically calculated by the software.

\subsection{Ex vivo Tissue Imaging}

To confirm that the observed fluorescence in Ova-sensitized and challenged mice after induction of AAI originates in the lungs and is not a fluorescence signal from another compartment (like the liver), NIR fluorescent images of excised lungs were record and analyzed as well. 


\subsection{Histology and immunohistochemistry}

\subsection{Preparation of lung sections}

To assess airway inflammation and mucus cell content in the airway wall, lungs were excised from the thorax and fixed in $4 \%$ paraformaldehyde overnight at room temperature. On the next day the samples were washed and incubated for 2 hours in PBS at room temperature. After this incubation time the lungs were dehydrated in dehydrationssystem device through an ascending ethanol gradient: in 70\%, $80 \%, 95 \%$ and $99 \%$ ethanol.

The dehydrated organs were incubated in xylene followed by incubation in liquid paraffin. Subsequently, the lungs were immersed and embedded in pure paraffin in small plastic molds. After the samples were cooled down to solidify the paraffin the lungs could be cut into 5- $\mu \mathrm{m}$ sections using a microtome (Leica). The sections were placed with the rough side on cold deionized water in a glass cuvette and after short incubation were smoothened on a $42{ }^{\circ} \mathrm{C}$ warm water bath. When the sections had lost their folds they were transferred onto Superfrost Plus glass slides (Roth) and fixed overnight at $56^{\circ} \mathrm{C}$ in an incubator.

To prepare cryo-sections of lung tissue the fresh biopsies was plunged into a suitable cryoagent and quickly frozen. After that the samples were placed on a cryostat chuck and could be embedded in a suitable embedding medium OCT. Then, cryo-sections were cut into $10 \mu \mathrm{m}$ sections with the help of a cryotom device.

\subsection{Hematoxylin-eosin staining}

Hematoxylin and eosin are one of the most important standard staining methods used in histology. It gives a structural overview of the tissue, allowing differentiation of the structures being classified as normal, inflamed or pathological. First, the nuclei are stained with a hematoxylin solution, appearing blue or dark violet. During the second step the tissue is counterstained with a xanthene dye, e.g. eosin Y, eosin B. In this process cytoplasm, collagen, keratin and erythrocytes are stained red (Llewellin et al., 2009).

To perform hematoxylin-eosin staining $5 \mu \mathrm{M}$ paraffin sections from the lungs were deparaffinized for $2 \times 15$ minutes in xylene and rehydrated through a descending ethanol gradient (2x5 minutes $99 \%$ ethanol, 2x5 minutes $96 \%$ ethanol, 2x5 minutes $70 \%$ ethanol 
following by incubation for 5 minutes in distillated water). After 10 minutes incubation in a hematoxylin solution the sections were washed in distillated water. To develop the color reaction the samples were incubated for 10 minutes under running tap water. Afterwards the sections were washed in distillated water and incubated for 5 minutes in a $0.1 \%$ eosin staining solution. After a quick washing step in distillated water the sections were dehydrated through an ascending ethanol gradient $(2 \times 5$ minutes $70 \%$ ethanol, $2 \times 5$ minutes $96 \%$ ethanol, 2x5 minutes $99 \%$ ethanol) and incubated for $2 \times 15$ minutes in xylene before they finally were mounted with Entellan under a cover glass.

\subsection{Anti-SiglecF immunohistochemistry}

The cryo-sections were washed for 10 minutes in Tris $(\mathrm{pH} 7.6)$, then for 10 minutes in Methanol containing $0.3 \% \mathrm{H}_{2} \mathrm{O}_{2}$, followed by two washing steps for 5 minutes in Tris. After that, the sections were incubated in Avidin solution for 10 minutes. The samples were washed in Tris for 5 minutes and incubated for 10 minutes in Biotin solution. After rinsing in Tris, unspecific binding was blocked by incubation with blocking Buffer-Fish for 15 minutes. Then the solution was taped from the sections, the sections were incubated with the rat anti-SiglecF antibody (1:50; BD, Heidelberg, Germany) overnight at $4{ }^{\circ} \mathrm{C}$ in the humid chamber. On the next day the sections were rinsed two times with Tris for 5 minutes, followed by incubation for 1 hour with the appropriate biotinylated secondary goat anti-rat antibody (1:2000; Dako, Hamburg, Germany). After that, the samples were washed with Tris two times for 5 minutes and were incubated for 1 hour at room temperature with Avidin-Peroxidase complex (1:400; Thermo Scientific, MA, USA). After rinsing the samples with Tris the sections were incubated with AEC ( $\mathrm{pH} 5.2)$ at room temperature for 30 minutes and stained with $\mathrm{HE}$ as a counterstain to the SiglecF-staining. Finally, the sections were dehydrated in an ascending ethanol gradient ( 2 minutes in distillated water, $2 \times 2$ minutes in $70 \%$ ethanol, 2x2 minutes $96 \%$ ethanol, 2x2 minutes $99 \%$ ethanol) followed by incubation for 2x15 minutes in xylene and mounted with Entellan under cover glass.

\subsubsection{Microscopy}

The stained paraffin-embedded sections or cryosections of the lung were inspected using a microscope fitted with a $10 x, 20 \times$ or $40 x$ objective lens. 


\subsubsection{ELISA}

\subsection{Cytokine ELISA}

To analyse the cytokine production of splenocytes their titers were determined by ELISA. The experiments were performed according to the manufacturer's instructions. Firstly, microtiter plates were coated with the respective capture antibody $(\mathrm{Ab})$ and incubated overnight at $4{ }^{\circ} \mathrm{C}$. On the next day the plates were washed with ELISA wash buffer and blocked with assay diluents for $1 \mathrm{~h}$ at the room temperature. The standards were prepared using assay diluent. Also the cell supernatans were diluted with assay diluent and added along with the standards to the plate after three washing steps. Standards and samples were incubated at the room temperature for $2 \mathrm{~h}$ with continuous shaking. After that the plates were washed and incubated with the biotin-labeled detection $\mathrm{Ab}$ for $1 \mathrm{~h}$ at room temperature. This was followed by three washing steps and incubation with the avidin-horse radish peroxidase mix for $30 \mathrm{~min}$ at room temperature. Finally, five washing steps were carried out followed by addition of the substrate buffer with TMB and $\mathrm{H}_{2} \mathrm{O}_{2}$. After 30 min of incubation the reaction was stopped using $1 \mathrm{M} \mathrm{H}_{2} \mathrm{SO}_{4}$ and the samples were measured using a photometer. Measurements were carried out at $450 \mathrm{~nm}$ and $570 \mathrm{~nm}$.

\subsection{Ovalbumin-specific Ig ELISA}

To analyse antigen-specific Ig production Ova-specific ELISAs were performed. Microtiter plates were incubated overnight at $4^{\circ} \mathrm{C}$ with $50 \mu \mathrm{g} / \mathrm{ml}$ Ova diluted in ELISA coating buffer. On the next day, the plates were washed three times with ELISA wash buffer and blocked for $1 \mathrm{~h}$ at room temperature with assay diluents to prevent unspecific binding to the plate. Serum samples were diluted with assay diluent. The dilution grade was dependent on the Ig isotype and on the genotype of the mice. To avoid IgG-contamination in $\operatorname{IgE}$ detection, the diluted samples were incubated with Protein G Sepharose to bind IgG in the serum samples. After three wash steps prepared the samples were added to the plate and incubated overnight at $4^{\circ} \mathrm{C}$. The next day unbound Igs were washed away by three wash steps and the detection $\mathrm{Ab}$ were added. After $1 \mathrm{~h}$ incubation at room temperature the plates were washed three times and the substrate buffer mix with 3,3',5,5'-Tetramethylbenzidine (TMB) and $\mathrm{H}_{2} \mathrm{O}_{2}$ could be added to develop the colour reaction based on the oxidation of TMB to tetramethylbenzidine diimin. The resulting diimine causes the solution to adopt a blue colour. The reaction was stopped using $1 \mathrm{M} \mathrm{H}_{2} \mathrm{SO}_{4}$ and the plates were measured using an ELISA Reader at $450 \mathrm{~nm}$ (product) and $570 \mathrm{~nm}$ (background). 


\subsubsection{Cell biological methods}

All cell biological experiments were carrying out under sterile conditions to avoid possible contamination.

\subsubsection{Determination of cell numbers}

The absolute cell number in cell suspensions was determinate using a Neubauer Counting chamber. First of all, the volume of the sample was measured and $10 \mu \mathrm{l}$ of cell suspension were taken out and mixed with PBS. This diluted cell suspension was vortexed briefly and $10 \mu 1$ of it were placed under the cover glass of the counting chamber. 4 x 16 fields were counted and the absolute cell number was calculated using the following formula:

$\left(\right.$ Counted cell number/4) $\mathrm{x}$ dilution factor $\times \mathrm{V}(\mathrm{ml}) \times 10^{4}$ (chamber factor) $=$ absolute cell number of sample

For the determination of the cell number of a single cell population in the BAL, the following calculation was used:

(Absolute cell number of the BAL) $\mathrm{x}$ (relative percentage [\%] of subpopulation of interest) $\mathrm{x}$ $0.01=$ absolute cell number of subpopulation

\subsubsection{Ovalbumin-specific restimulation of splenocytes ex vivo}

To examine the antigen-specific proliferation of T and B cells, 300.000 splenocytes were cultured for $72 \mathrm{hrs}$ in round-bottom 96 -well plates at $37{ }^{\circ} \mathrm{C}$ and $5 \% \mathrm{CO}_{2}$. The cells were incubated in $200 \mu 1$ complete RPMI medium supplemented with $10 \%$ heat-inactivated FCS and $1 \%$ penicillin/streptomycin with or without the addition of Ovalbumin $(10 \mu \mathrm{g} / \mathrm{ml})$. As a positive control proliferation after addition of Concanavalin A (ConA) at a concentration of $2.5 \mu \mathrm{g} / \mathrm{ml}$ was used.

After the culture, $50 \mu \mathrm{l}$ of supernatant were removed from the plate and frozen at $-20{ }^{\circ} \mathrm{C}$ for ELISA analysis of produced cytokines, whereas the cells were cultured for another $16 \mathrm{hrs}$ with ${ }^{3} \mathrm{H}$-Thymidin $\left(1 \mu \mathrm{Ci}\right.$ per well) at $37{ }^{\circ} \mathrm{C}$ and $5 \% \mathrm{CO}_{2}$. On the next day the cells were soaked into a filter (Printed Filtermat A) using Filtermate Harvester, and a MeltiLex A scintillator sheet was molten onto the filter in the microwave at $800 \mathrm{~W}$ for 2 minutes. The radioactivity was then measured as cpm with a scintillation counter device Microplate Counter 2450 Microbeta $^{2}$. 


\subsubsection{Collection of samples}

The mice were euthanized via $\mathrm{CO}_{2}$ inhalation and nebulised with $70 \%$ ethanol, followed by exposure of the peritoneum cavity. Afterwards the BAL, spleen, blood, lung tissue and tail biopsy were collected.

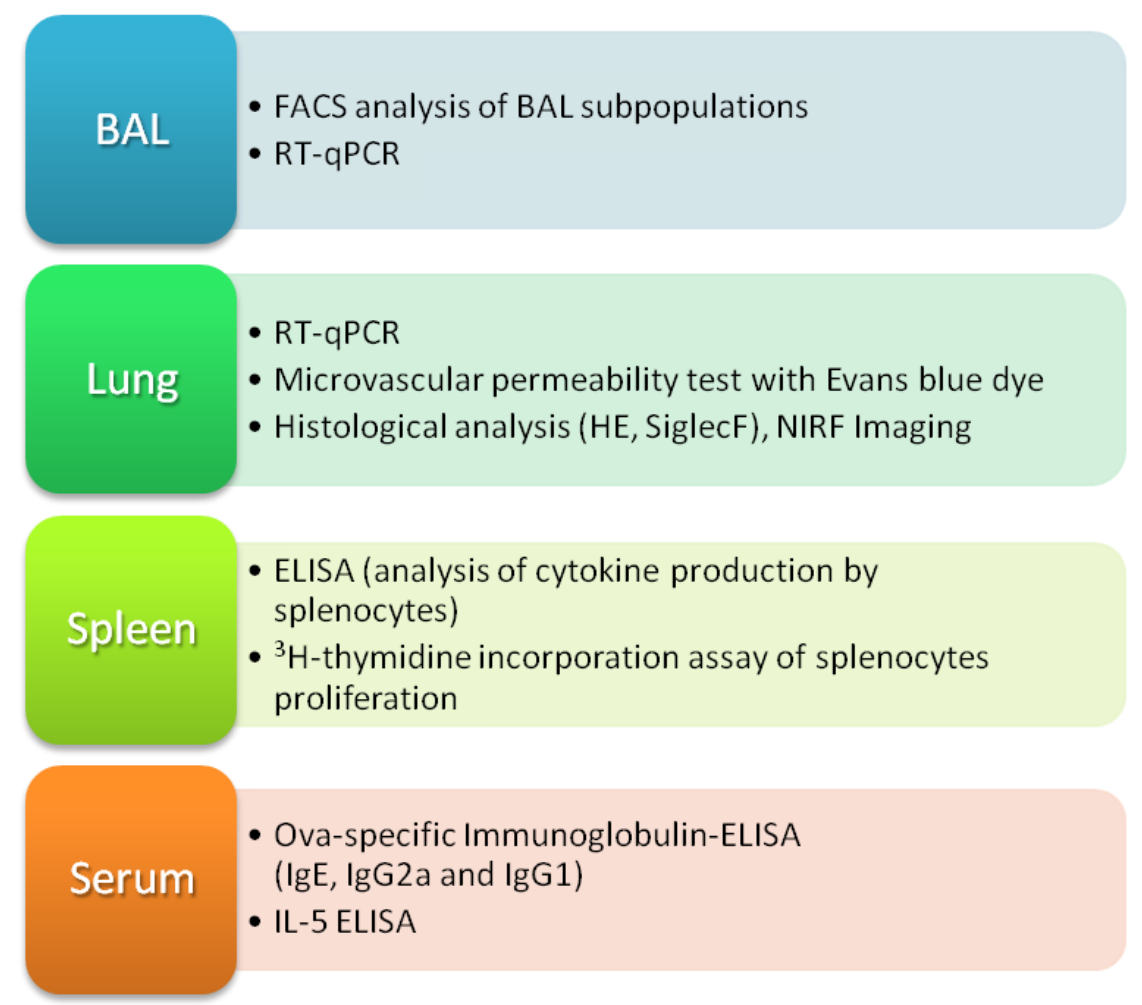

Schema of sample collection. To investigate the modulation of the inflammatory response by GCtherapy the following methods were applied: FACS, RT-qPCR, ELISA, ${ }^{3} \mathrm{H}$-thymidine assay, microvascular permeability test, histology and NIRF Imaging in vivo and ex vivo.

\subsection{Isolation of splenocytes}

To isolate lymphocytes from the spleen for proliferation assay, the freshly isolated organs were passed through a $40 \mu \mathrm{m}$ cell strainer and resuspended in PBS. The cell suspension was centrifuged for 7 minutes at $300 \mathrm{~g}$ and $4{ }^{\circ} \mathrm{C}$. After removing the supernatant the cell pellet was resuspended in $1 \mathrm{ml}$ PBS and incubated with $6 \mathrm{ml}$ TAC buffer for 12 minutes. To stop this reaction $25 \mathrm{ml}$ PBS was added to the tube and the sample was centrifuged for 7 minutes at $300 \mathrm{~g}$ and $4{ }^{\circ} \mathrm{C}$. The resulting cell pellet was resuspended in $1 \mathrm{ml}$ PBS containing $0.01 \%$ BSA.

\subsection{Isolation of the bronchoalveolar lavage (BAL)}

BAL isolation was performed about $40 \mathrm{hrs}$ after the last intranasal Ovalbumin challenge. The mice were euthanized via $\mathrm{CO}_{2}$ inhalation and nebulised with $70 \%$ ethanol, followed by 
exposure of the peritoneal cavity and the diaphragm. After removing the skin and thyroid gland an indwelling cannula was inserted into the trachea. Afterwards the BAL was collected by gentle flushing the lungs three times with $1 \mathrm{ml}$ aliquots of cold sterile PBS/BSA solution. The recovered cell suspension was centrifuged for 6 minutes at $350 \mathrm{~g}$ at $4^{\circ} \mathrm{C}$. After removing the supernatant the BAL cells were resuspended in $1 \mathrm{ml}$ PBS and incubated with TAC buffer for 12 minutes at room temperature to lyse erythrocytes in the sample. Then cells were then washed with PBS/BSA for 6 minutes at $350 \mathrm{~g}$ and $4^{\circ} \mathrm{C}$. Afterwards the supernatant was removed and the cells were resuspended in the reflux. The total volume of the BAL samples and the absolute cell numbers were determined. One part of cell suspension was used for the analysis of the cell composition via FACS. Another part was used for RNA isolation, followed by reverse transcription and gene expression analysis via RT-qPCR.

\subsection{Isolation of serum}

After the exposure of the peritoneal cavity and the diaphragm, blood sample was isolated from the heart using cannula injection in the left and right heart ventricle. The isolated blood was collected into a centrifugation tube. After incubation at room temperature samples were centrifuged for 30 minutes at full speed and room temperature. Afterwards the liquid transparent supernatant was carefully transferred into a new centrifugation tube and stored at $-20{ }^{\circ} \mathrm{C}$ until analysis.

\subsection{Isolation of the lungs}

After isolation of the BAL the upper torso region was opened and the lung was taken out. Then thymus, heart, lymph nodes and intestinal tract were separated from the lungs. The lungs were used for histology and gene expression analysis. For histology half of the freshly isolated lungs was incubated with $4 \%$ paraformaldehyde overnight at room temperature. The other half of the lungs was transferred into a new tube right after isolation and quickly frozen in solid carbon dioxide snow. Then the samples were stored at $-80^{\circ} \mathrm{C}$ until RNA isolation.

\subsection{Epithelial cell isolation from the lung}

To isolate epithelial cells mice were sacrificed by $\mathrm{CO}_{2}$ inhalation. After the peritoneal cavity and diaphragm were exposed blood was flushed from the lungs by perfusion with $20 \mathrm{ml}$ icecold PBS with $5 \mathrm{mM}$ EDTA. To achieve this, a small cut was done into the apex cordis and a thin cannula was placed into the right ventricle of the heart. Then blood was rinsed out with 
constant pressure until the lungs changed colour to white and were visually free of blood. In the next step the BAL was isolated from the lungs to avoid contamination of the epithelial cells by infiltrating cells. After that the trachea was intubated by an indwelling cannula and the lungs were filled with $1 \mathrm{ml}$ of pre-warmed $\left(37^{\circ} \mathrm{C}\right)$ Dispase $(50 \mathrm{U} / \mathrm{ml})$. Then pre-warmed low melt agarose $\left(1 \%, 0.5 \mathrm{ml}\right.$, stored in a $45^{\circ} \mathrm{C}$ water bath) was infused slowly via the catheter to seal the airways and thereby to prevent leaking out of the Dispase. The lungs were immediately covered with crushed ice and incubated for $2 \mathrm{~min}$. Afterwards, the lungs were moved into $2 \mathrm{ml}$ of pre-warmed Dispase in a $15 \mathrm{ml}$ tube and incubated for 20 minutes at room temperature. Then the lungs were transferred to a $60-\mathrm{mm}$ Petri dish and cut with the scissors in small pieces (approximately $1 \mathrm{~mm}^{2}$ ) and placed in incubator for another 20 minutes at $37^{\circ} \mathrm{C}$. Afterwards $10 \mathrm{ml}$ of DMEM with $100 \mu \mathrm{l}$ DNase (2000 U) solution was added to the tissue. The digested tissue was placed on a shaker for 10 minutes at room temperature. The resulting suspension was filtered through $100 \mu \mathrm{M}$ and then $40 \mu \mathrm{M}$ cell strainers into $50 \mathrm{ml}$ tube and washed intensively with DMEM to avoid a loss of cells. The filtered suspension was centrifuged at $130 \mathrm{~g}$ for 10 minutes at $4^{\circ} \mathrm{C}$. After centrifugation the supernatant was removed and the cell pellet was resuspended in the reflux. To avoid contamination with remaining erythrocytes the lung cell suspension was incubated with TAC-buffer (as described above) followed by a washing step in FACS Buffer (130 g for 10 minutes and $\left.4{ }^{\circ} \mathrm{C}\right)$. After this centrifugation step, the cell pellet was resuspended in the reflux and the cell number was determined. To further purify the resulting epithelial cell fraction an immunoisolation techniques via FACS-Sorting was used.

\subsubsection{Analytical FACS analysis}

All FACS measurements were performed using a FACSCanto device. Data analysis was performed with the program FlowJo. To analyze differential cell compositions of murine bronchoalveolar lavage fluid by FACS the isolated cells were stained with a combination of commercially available fluorochrome-labelled antibodies: CD3-PerCP, CD4-APC, SiglecFPE, F4/80-FITC, CD11b-PE-Cy7 and GR1-APC-Cy7. This combination of antibodies allows identifying T lymphocytes, macrophages and monocytes, eosinophils and neutrophils in BAL.

Before the BAL samples were analysed via FACS, a compensation setup was made and the FACS antibody-combination was tested to avoid spectral overlap of the fluorochromes.

For FACS analysis of the cell population composition in the BAL approximately $1 \times 10^{5}-2 \times 10^{5}$ cells were transferred into FACS tubes and washed with $4 \mathrm{ml}$ of FACS Buffer 
for 5 minutes at $350 \mathrm{~g}$ and $4^{\circ} \mathrm{C}$. After removing of the supernatant, the cell pellet was incubated with $20 \mu \mathrm{Fc}$-Block antibody (anti-CD16/CD32) at $4^{\circ} \mathrm{C}$ for 15 minutes to avoid nonspecific staining of the cells. Then the antibody cocktail was added to the cell suspension and after briefly vortexing the cells were stained for another 20 minutes at $4^{\circ} \mathrm{C}$. Subsequently the cells were washed with $4 \mathrm{ml}$ FACS Buffer and centrifuged for 5 minutes at $350 \mathrm{~g}$ and $4^{\circ} \mathrm{C}$. After removing of the supernatant the cell pellet was resuspended in the reflux and analysed using the FACS device (BD FACS Canto II).

\subsection{Cell sorting via FACS}

To isolate eosinophils from the BAL, the cells were stained with the antibodies cocktail against $\mathrm{T}$ lymphocytes, macrophages and monocytes, eosinophils and neutrophils (as described above) after pre-incubation with Fc-block. The sorting of eosinophils was performed by positive selection of the cell population, which was marked with the eosinophils cell surface marker SiglecF.

To isolate epithelial cells from the lung of wild type and knockout mice the cell suspension was pre-incubated for 15 minutes with Fc-block and stained for another 20 minutes at $4{ }^{\circ} \mathrm{C}$ with the following combination of fluorochrome-labelled antibodies: Podoplanin-PE, CD34PeCy5, CD45.2-APC and F4/80-FITC. This combination of antibodies identifies endothelial cells, stromal cells (fibroblasts), macrophages and all other hematopoietic cells. The isolation of epithelial cells was performed by negative selection because these cells should be not marked with antibodies against hematopoietic cells or stroma cells which are the possible contamination of the isolated epithelial cell suspension from the lungs. After gating the cell populations of interest, epithelial cells and eosinophils could be collected into the FACS tubes and placed on the ice until RNA isolation. 


\subsubsection{Molecular biological techniques}

\subsubsection{Isolation of RNA from lung tissue}

Pieces or whole deep-frozen lung tissues was homogenized in $900 \mu 1$ Qiasol Lysis Reagent with a tissue homogenisator. The lysates were transferred into Eppendorf tubes and incubated for 5 minutes at room temperature to initiate the dissociation of the nucleoprotein-complexes. To reduce possible contamination with genomic DNA, $100 \mu 1$ of gDNA Eliminator Solution was added to each sample and mixed for 15 second on a vortexer. Afterwards, $180 \mu \mathrm{l}$ chloroform was added to the samples. The resulting homogenates were vortexed for 15 seconds and incubated for 3 minutes at room temperature. The tubes were centrifuged for $15 \mathrm{~min}$ at $4^{\circ} \mathrm{C}$ at full speed; thereby the sample separated into three phases: an upper, colourless, aqueous phase containing RNA; a white interphase; and a lower, red, organic phase. The $600 \mu \mathrm{l}$ of the aqueous upper phase including the RNA was transferred into a new tube and mixed with $600 \mu 170 \%$ ethanol by pipetting up and down. After that, samples were transferred to an RNeasy Mini spin column placed in a $2 \mathrm{ml}$ collection tube and centrifuged for 20 seconds at full speed at room temperature. Then $700 \mu 1$ Buffer RWT were added to the column and centrifuged for another 20 second at $8000 \mathrm{~g}$ to wash the membrane. After this centrifugation step $500 \mu$ l Buffer RPE were added to the column and centrifuged for 3 minutes and $8000 \mathrm{~g}$ to wash the membrane and dry the spin column membrane, ensuring that no ethanol was carried over during RNA elution. After that, the RNeasy spin column was placed in a new collection tube and $35 \mu \mathrm{l}$ of RNase-free water were added directly to the spin column membrane. To elute the RNA, the samples were centrifuged for 1 minute at $8000 \mathrm{~g}$. To obtain more RNA another $35 \mu \mathrm{l}$ of RNase-free water were added into the RNease spin column and centrifuged once more for 1 minute. The obtained RNA was used immediately or stored at $-20^{\circ} \mathrm{C}$ for future use.

\subsubsection{Isolation of RNA from cells}

To isolate RNA from BAL cells or epithelial cells the Mini RNA Isolation Kit was used. This kit is designed for rapid isolation of total RNA from small amounts of cells. The cell samples were collect by gentle centrifugation at $350 \mathrm{~g}$ for 5 minutes and the supernatant was removed. $600 \mu \mathrm{l}$ of RNA ZR Extraction Buffer were added to the samples and mixed briefly by 15 seconds of vortexing. Afterwards the mixture was transferred to a Zymo-Spin Column and centrifuged at full speed for 1 minute into a $2 \mathrm{ml}$ Collection Tube. The flow-through from the collection tube was emptied whenever necessary to prevent contamination of the 
column by the flow-through. In the next step $400 \mu \mathrm{l}$ of Pre-Wash buffer were added to the Zymo-Spin Column and centrifuged at full speed for 1 minute to wash. Another $700 \mu 1$ of Wash buffer was added to the Zymo-Spin Column and the centrifugation step was repeated twice as above. Afterwards the column was transferred to a new RNase-free $1.5 \mathrm{ml}$ tube. After adding $40 \mu \mathrm{l}$ DNase and RNase free water directly to the column membrane, the samples were incubated for 1 minute at room temperature and spun briefly to elute the RNA. The obtained RNA was used immediately or stored at $-20^{\circ} \mathrm{C}$ for future use.

\subsubsection{Purification of RNA}

For RNA purification the RNeasy Mini Kit II (Quiagen, Hilden, Germany) was used. Firstly, the prepared RNA was incubated with a DNaseI containing mixture (10 $\mu 110 \times$ DNase Ibuffer containing $1 \mu \mathrm{l}$ DNase I) for 30 minutes at $37^{\circ} \mathrm{C}$. Afterwards $350 \mu \mathrm{RLT}$ buffer (containing $10 \mu \mathrm{l}$ of $\beta$-Mercaptoethanol per $1 \mathrm{ml}$ RLT solution) were added into the centrifuge tube containing the RNA sample. After briefly vortexing $250 \mu \mathrm{l}$ of absolute Ethanol $(96 \%)$ were mixed with the sample by gentle pipetting up and down. In the next step the resulting solution was added on the RNeasy Column and centrifuged at full speed for 30 seconds into a $2 \mathrm{ml}$ Collection Tube. The flow-through from the collection tube was emptied. The sample was washed twice with $500 \mu \mathrm{l}$ of RPE buffer for 30 seconds at full speed and $4^{\circ} \mathrm{C}$ followed by an eluation step with DEPC-water. For that $30 \mu 1$ of DEPC-water were added directly to the column membrane and centrifugated for 1 minute at full speed and $4^{\circ} \mathrm{C}$ after 1 minute of incubation at room temperature. The obtained RNA was measured with a Nano Drop device to analyze the nucleotide concentration and quality. As another RNA quality control test the samples were mixed with OrangeG and run on a $1.5 \%$ agarose gel. The obtained RNA was used immediately or stored at $-20^{\circ} \mathrm{C}$ for future use.

\subsubsection{Reverse transcription of RNA}

To generate complementary DNA (cDNA) from RNA templates, reverse transcription was done with help of the iScript ${ }^{\mathrm{TM}} \mathrm{cDNA}$ Synthesis Kit. This kit provides all required reagents to create first-strand cDNA. Because all kit components are optimized to facilitate efficient cDNA synthesis using 1 pg to $1 \mu \mathrm{g}$ of total RNA samples were diluted with DNase/RNasefree-water to obtain this concentration. 
The iScript reverse transcriptase mixture contains a recombinant RNase H+ MMLV reverse transcriptase and a recombinant RNase inhibitor. This unique blend of buffers, stabilizers, and dNTPs streamlines reaction setup and ensures robust synthesis of first-strand cDNA (adopted from iScript ${ }^{\mathrm{TM}}$ cDNA Synthesis Kit manufacture instructions).

A master mix with all components, except the RNA was prepared and then dispensed into each tube with RNA on ice.

\begin{tabular}{l|r} 
Components & Volume \\
\hline Nuclease-free water & variable \\
5x iScript select reaction mix & $4 \mu 1$ \\
iScript reverse transcriptase & $0.35 \mu 1$ \\
RNA sample (1 pg to 1 $\mu$ g total RNA) & variable \\
\hline Total volume & $20 \mu 1$
\end{tabular}

All components were gently mixed and incubated in a Thermocycler as follows:

\begin{tabular}{l|r} 
Duration and temperature $\left({ }^{\circ} \mathbf{C}\right)$ & Function \\
\hline 5 minutes at $25^{\circ} \mathrm{C}$ & Binding of Oligonucleotides \\
30 minutes at $42^{\circ} \mathrm{C}$ & cDNA synthesis by reverse transcription \\
5 minutes at $85^{\circ} \mathrm{C}$ & Heat-inactivation of reverse transcriptase
\end{tabular}

The resulting cDNA product was used directly for PCR amplification or stored at $-20^{\circ} \mathrm{C}$ (for long periods) or $4{ }^{\circ} \mathrm{C}$ (for direct analysis).

\subsubsection{PCR}

In this work PCR was applied in genotyping of animals and examination of reconstitution efficiency after bone marrow transplantation, but also to test the DNA and cDNA quality before gene expression analyses via RT-qPCR. In the last case, the DNA/cDNA quality was tested via PCR analysis of the housekeeping gene $\beta$-Actin or HPRT. To do this, the DNA was mixed with sequence-specifically oligonucleotides, Taq polymerase, nucleotides, water and $\mathrm{MgCl}_{2}$-containing PCR buffer. 


\begin{tabular}{l|r}
\hline Components & Volume \\
\hline cDNA/ DNA (genomic) & $1 \mu \mathrm{l}$ \\
(undiluted BAL cDNA; lung cDNA in 1:10-dilution) & \\
Oligonucleotids & $0.5 \mu 1$ (from $10 \mu \mathrm{M} \mathrm{mix)}$ \\
dNTP & $1 \mu \mathrm{l}(5 \mathrm{mM})$ \\
Taq-Polymerase & $0.2 \mu \mathrm{l}(5 \mathrm{U} / \mu \mathrm{l})$ \\
$10 x P C R-P u f f e r\left(\right.$ with $\left.1.5 \mathrm{mM} \mathrm{MgCl}_{2}\right)$ & $2.5 \mu \mathrm{l}$ \\
$\mathrm{H}_{2} \mathrm{O}$ & $19.8 \mu \mathrm{l}$ \\
\hline Total volume & $\mathbf{2 5} \boldsymbol{\mu l}$
\end{tabular}

The PCR analysis involves the following heating and cooling steps:

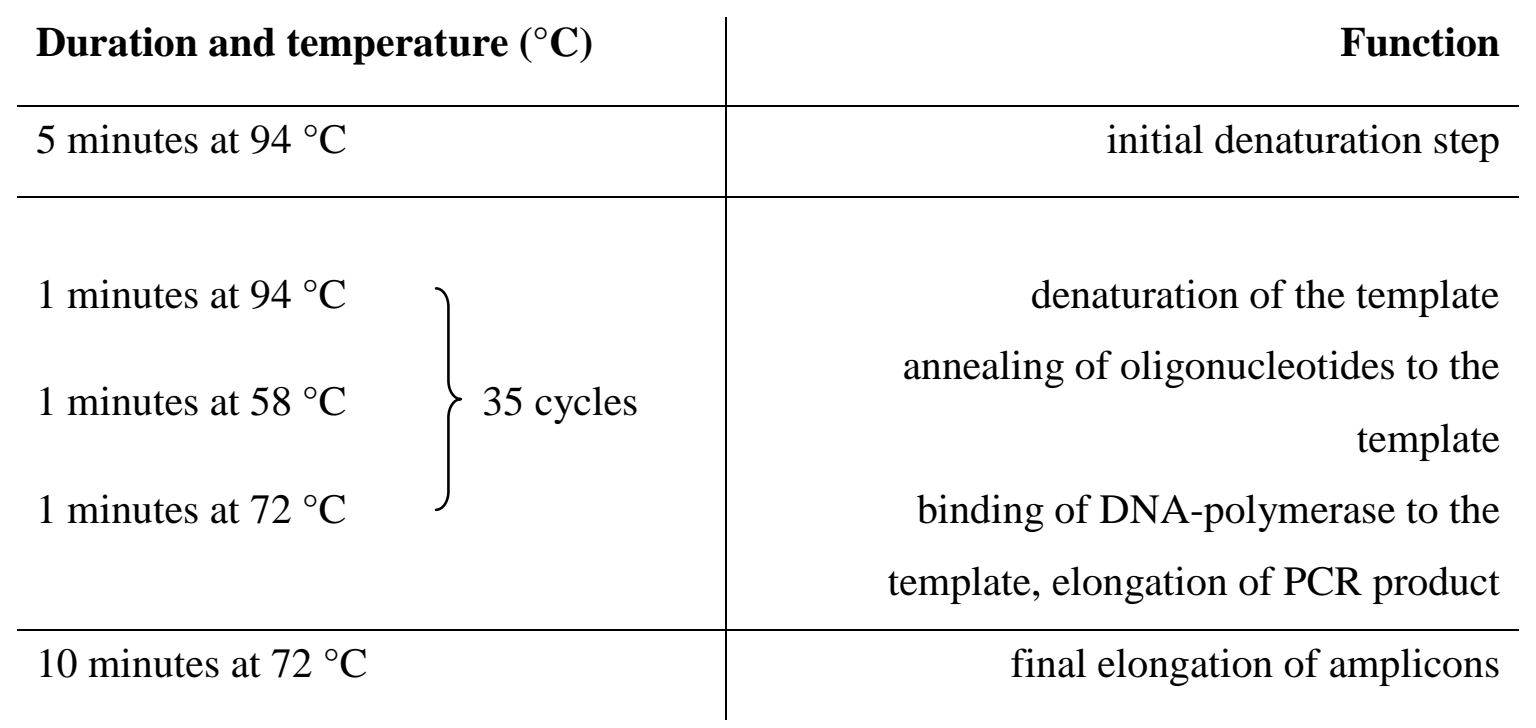

Termination of the PCR was achieved by chilling the sample mixture to $4{ }^{\circ} \mathrm{C}$ in the thermocycler. The resulting amplicons were analysed by agarose gel electrophoresis. All PCR analyses were performed using a thermocycler device.

\subsection{Quantitative reverse transcription PCR (RT-qPCR)}

In the present work the $\Delta \Delta \mathrm{Ct}$ method was used for relative quantification of gene expression. As an endogenous housekeeping gene Hypoxanthine-guanine phosphoribosyltransferase (HPRT) was chosen because this gene is constitutively expressed at low levels which is useful for RT-qPCR analysis. 
All RT-qPCR analyses were performed using an ABI 7500 instrument (Applied Biosystems, Darmstadt, Germany). The analyses were run according the following program:

\begin{tabular}{l|r} 
Duration and temperature $\left({ }^{\circ} \mathbf{C}\right)$ & Function \\
\hline $\begin{array}{l}2 \text { minutes at } 50{ }^{\circ} \mathrm{C} \\
10 \text { minutes at } 95^{\circ} \mathrm{C}\end{array}$ & $\begin{array}{r}\text { Initial denaturation step } \\
\text { and enzyme activation }\end{array}$ \\
\hline 15 seconds at $95{ }^{\circ} \mathrm{C}$ \\
$\begin{array}{l}1 \text { minute at } 60{ }^{\circ} \mathrm{C} \\
15 \text { seconds at } 95^{\circ} \mathrm{C}\end{array}$ \\
1 minute at $60{ }^{\circ} \mathrm{C}$ & Denaturation of the template \\
15 seconds at $95{ }^{\circ} \mathrm{C}$ & Annealing, Amplification \\
15 seconds at $60^{\circ} \mathrm{C}$ & Denaturation of DNA \\
\hline
\end{tabular}

The cDNA samples were added to a 96-well plate and mixed with the following components:

\begin{tabular}{l|r} 
Components & Volume \\
\hline cDNA & $1 \mu \mathrm{l}$ \\
Oligonucleotides & $0.5 \mu \mathrm{l}$ (from $10 \mathrm{pM}$ mix) \\
SYBR Green reaction mix & $12.5 \mu \mathrm{l}$ \\
$\mathrm{H}_{2} \mathrm{O}$ & $11 \mu \mathrm{l}$ \\
\hline Total volume & $25 \mu \mathrm{l}$
\end{tabular}




\subsubsection{Genotyping of bone marrow chimeras}

\subsection{DNA extraction from the blood and tail biopsy}

For genotyping DNA was extracted from blood cells or tail biopsy. To isolate blood cells from mice, a small piece of the tail was cut off (and used for tail analysis) and 3 blood drops were collected in $1.5 \mathrm{ml}$ Alservers into centrifugation tubes. Afterwards the samples were centrifuged for 5 minutes at $350 \mathrm{~g}$ and $4{ }^{\circ} \mathrm{Cand}$ the supernatant was removed. Cell pellets of blood samples or tail biopsies of the mice were digested in $750 \mu 1$ tail buffer containing $20 \mu \mathrm{l}$ Proteinase $\mathrm{K}$ at $56^{\circ} \mathrm{C}$ overnight. On the next day the samples were mixed with $280 \mu \mathrm{l}$ saturated $\mathrm{NaCl}$ solution for 5 minutes and centrifuged for 10 minutes at room temperature and full speed. In the next step $850 \mu 1$ of the upper phase was mixed with $600 \mu 1$ Isopropanol. After 3 minutes of incubation the sample was centrifuged for 10 minutes at room temperature and full speed. After removing the supernatant the RNA pellet was washed with $500 \mu \mathrm{l}$ of $70 \%$ ethanol and centrifuged again for 10 minutes. The supernatant was carefully removed and the pellet dried at room temperature. In the last step the pellet was dissolved in $100 \mu \mathrm{l}$ TE-Puffer and analyzed by PCR or stored at $4{ }^{\circ} \mathrm{C}$.

\subsection{Reconstitution efficacy analysis}

Reconstitution efficacy of bone marrow chimeras was analyzed by monitoring the peripheral blood chimerism six weeks after transplantation. To test the reconstitution efficiency of $\mathrm{GR}^{\mathrm{dim}}$ bone marrow chimeric mice blood samples and tail biopsy were genotyped by PCR with primers located in exons 3 and 5 of the GR gene (like described before by Reichardt et al., 1998). PCR reactions were performed as described above. To distinguish between both alleles, an aliquot of the resulting PCR product was digested by incubation with the enzyme BsrGI for 2 hrs at $37^{\circ} \mathrm{C}$. Afterwards, the digested sample was separated on a $1.5 \%$ agarose gel, and visualization of the bands was achieved using the ChemoStar gel imager (Intas $\mathrm{GmbH})$. The wild-type allele shows a band at $0.24 \mathrm{~kb}$ whereas the $\mathrm{GR}^{\mathrm{dim}}$ allele shows two bands near the size of $0.12 \mathrm{~kb}$. The appearance of two instead of one band is due to a new restriction site for BsrGI introduced by the point mutation in the $\mathrm{GR}^{\mathrm{dim}}$ mice. Based on this analysis the efficacy of reconstitution could be successfully verified. 


\subsubsection{Agarose gel electrophoresis}

DNA or RNA samples were separated by (1\% or $2 \%)$ agarose gel electrophoresis. The gels were prepared by dissolving the agarose in the 1 xTAE buffer. To visualize the DNA, $50 \mu 1$ of $(10 \mathrm{mg} / \mathrm{ml})$ ethidium bromide were added to $450 \mathrm{ml}(1 \%$ or $2 \%)$ agarose solution. DNA or RNA samples were mixed with $6 \times$ OrangeG Loading Buffer before loading on the gel. Electrophoresis was performed at $120 \mathrm{~V}$ for 35 Minutes. Visualization of the bands was performed using a UV light with UV System camera and a gel imager device.

To determine the fragment sizes of the analyzed samples, a DNA molecular weight marker (standard $1 \mathrm{~kb}$ DNA ladder) was used.

\subsubsection{Statistical analysis}

Statistical evaluation was performed using the GraphPad Prism 5 and 6 Software (GraphPad Software). The 2way ANOVA test and Bonferroni test were chosen to compare two groups, if not otherwise stated.

Results are presented as mean \pm SEM (standard error of the mean). Based on the calculated p-value the significance of the data could be analyzed: $p$-values $<0.05$ were considered to indicate statistical significance, $p<0.05(*), p<0.01(* *), p<0.001(* * *)$ and $p<0.0001$ $(* * * *)$. 


\section{Results}

\subsection{Establishment of AAI as a mouse model of human Asthma bronchiale}

Asthma is defined as a chronic inflammatory disease of the airways induced by a of $\mathrm{T}_{\mathrm{H}} 2$ immune response (Gina et al., 2006; Bousquet et al., 2000). The selection of the $\mathrm{T}_{\mathrm{H}} 2$ inflammatory pathway is initiated by the uptake of allergens by professional APCs that present selected peptides on MHCII molecules to naïve T cells. The antigen presentation by APCs directs the specification of naive $T$ cells in favour of a $T_{H}$ 2-cell phenotype accompanied by the production of several $\mathrm{T}_{\mathrm{H}} 2$ cytokines, chemokines and other mediators that leads to lung infiltration by inflammatory cells (e.g. eosinophilia), production of immunoglobulins (IgE, IgG1), cytokine production and hypersecretion of mucus by epithelial cells of the airways (Vignola et al., 2000, 2003; Hammad et al., 2008).

To induce allergic airway inflammation (AAI) in the lungs of mice chicken-egg Ovalbumin (Ova) was used as an allergen because of its feature to induce a robust, allergic response in laboratory rodents (Kumar et al., 2008). For the induction of AAI, mice were immunized by intraperitoneal injection once a week for 4 consecutive times with Ova emulsified in aluminium hydroxide (Alum) as a $\mathrm{T}_{\mathrm{H}} 2$ driving adjuvant or with Alum alone (control group). Four weeks after immunization, the mice were challenged by an intranasal application of Ova. One group of Ova-immunized and challenged mice remained untreated whereas another group was treated with Dexamethasone intraperitoneally. About 40 hours after the last challenge the mice were sacrificed. The BAL was isolated from the lungs and prepared for analysis of the cell composition by FACS and for RNA isolation. The spleen was isolated to test for proliferation and priming ex vivo; blood was collected for the determination of immunoglobulin and IL-5 production; the lung tissue was isolated for histological and gene expression analyses.

\subsubsection{Histological analysis of lung inflammation in wild type mice}

In order to investigate inflammation in the lungs after induction of the allergic response, the lung tissue was analysed for characteristic cellular and structural changes. Based on the analysed lung sections from healthy and asthmatic mice the mouse model of asthma that was used in this work leads to a strong infiltration of the lungs by lymphocytes and to hyperplasia of epithelial cells with increased mucus secretion. 
This observation is based on the histological analysis of lung tissue from healthy and AAI mice by HE-staining (Figure 12) and PAS-staining (data not shown).

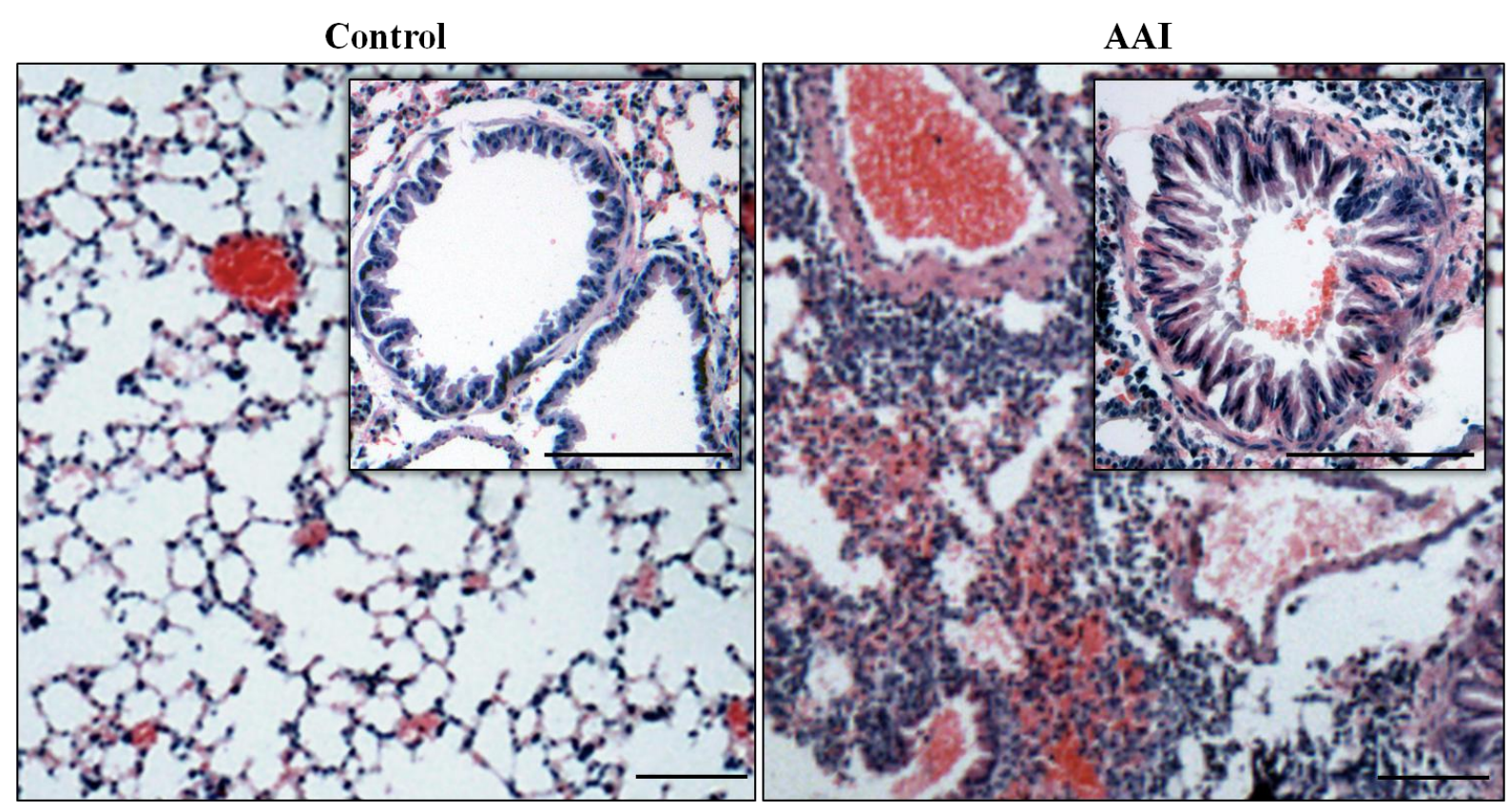

Figure 12: Histological analysis of the lung structure by HE-staining of paraffin sections from healthy (left) and AAI (right) mice. The lungs were collected $40 \mathrm{hrs}$ after the last challenge with Ova. After fixation in PFA the lungs were cut in $4 \mu \mathrm{m}$ sections and stained with HE. The left pictures represent the lung parenchyma (at 10x magnification) and conducting airways (at 40x magnification) of a healthy control mouse. The right pictures demonstrate typical inflammed lung tissue (at 10x magnification) and bronchioli with mucus hypersecretion and hyperplasia of goblet cells (at 40x magnification). The scale bars in the lower right corner of all images equal $100 \mu \mathrm{m}$.

The healthy lung parenchyma has a "spongy" structure and includes, without limitations, thin-walled alveoli that are specialized for gas exchange, blood vessels and conducting airways. On the histological sections only small numbers of lymphocytes can be seen in the lung parenchyma.

The lung tissue of mice with an asthma-like reaction shows strong infiltration by different lymphocytes and alveolar collapse. The parenchymal structure differs in free airspace because of cell influx into the lungs and mucus hypersecretion by the airway epithelium.

In the mouse model used in this work, lung remodelling did not occur because of the short time of the allergic reaction, but on the histological section one can see first signs of airway deformation based on goblet cell hyperplasia, thicker muscle layer around the bronchi and increased numbers of blood vessels (Figure 12). The bronchioles in the lung section of AAI mice are constricted with a cellular infiltrate in the airways that is absolutely absent in the lungs of healthy mice. 


\subsubsection{Establishment of the gating strategy for flow cytometric analysis of the BAL}

The cell composition of the BAL fluid was analysed by FACS. Because the cell populations in BAL differ in size, granularity and the expression of different cell type-specific surface markers these characteristics can be used to determine the cell composition.

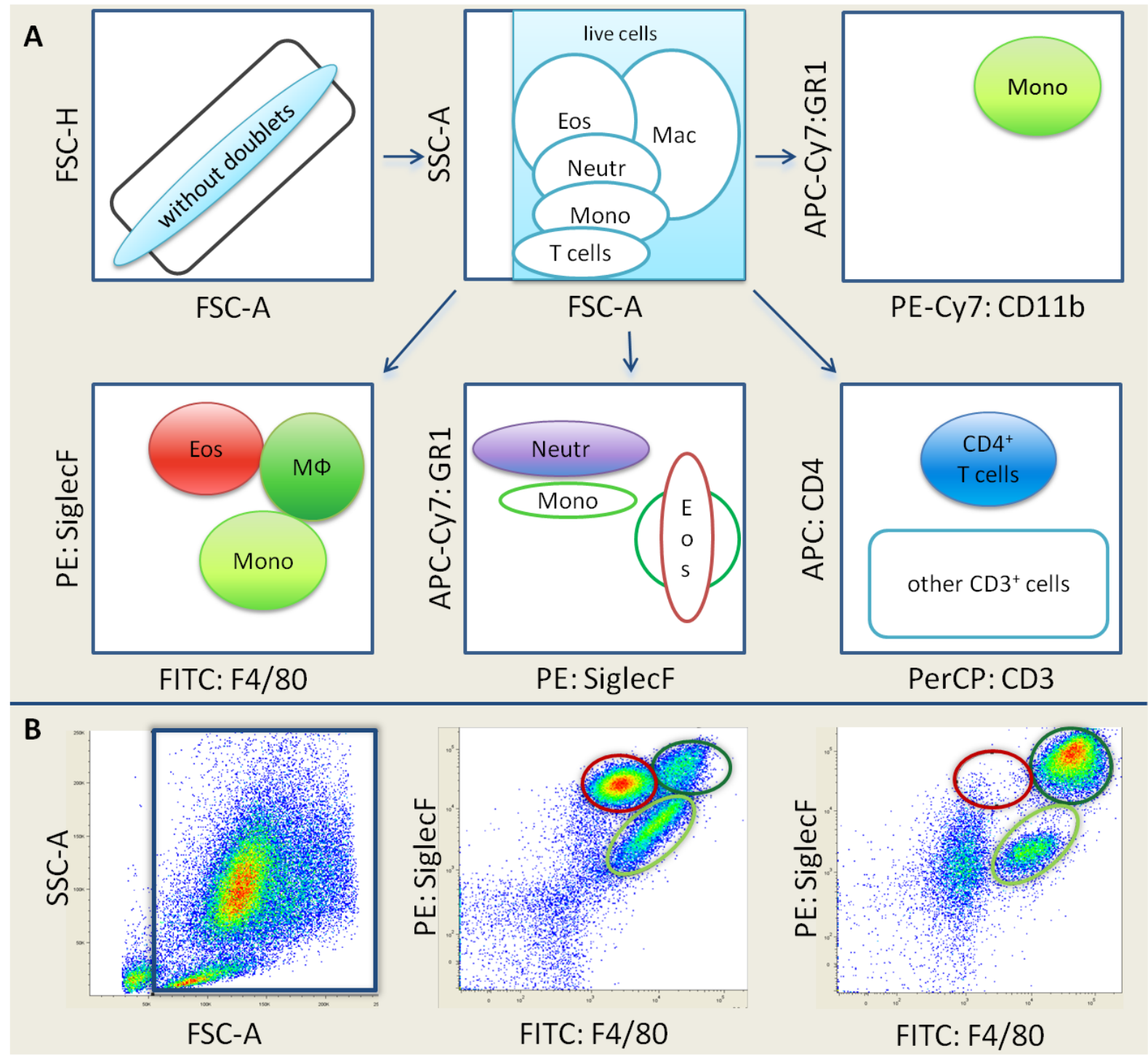

Figure 13: Gating strategy for flow cytometric analysis. (A) To analyse the cell composition of the BAL from control and Ova-sensitized mice with or without GC-treatment, the analysed cells were first gated for singlets (FSC-H vs. FSC-A) and then for live lymphocytes (SSC-A vs. FSC-A, colored in light blue). The resulting live cells-gate was further analyzed for the expression of cell populationspecific markers. The eosinophil gate was defined as SiglecF high, F4/80 low population (colored in red); the macrophages as F4/80 high, SiglecF positive cells (colored in deep green); the monocytes as SiglecF low, F4/80 high cells (colored in light green); the neutrophils as GR1 high, SiglecF negative cells (colored in violet); T helper cells as CD4 high, CD3 high cell population (colored in blue). (B) Representative lymphocyte plots for one AAI mouse (SSC-A vs. FSC-A plot and SiglecF vs. F4/80 plot) and for a healthy control mouse (SiglecF vs. F4/80 plot, right picture) 
First of all, it is important to separate the cells from „doublets“. Cell-,,doublets“" show the same FSC-Height but a bigger FSC-Area (more than from one cell). These "false" cells can be excluded via gating only on the cells that are located in diagonal position.

Because the BAL contains different population with diverse cell size and granularity, eosinophils, macrophages and T cells can be identified on these FSC/SSC plot (Figure 14). However, due to the overlapping of these populations it is impossible to reliably analyse the BAL cell composition without using fluorochrome-labelled antibodies. Therefore the isolated BAL was prepared for FACS analysis as described before. The cells were stained with Fc block to avoid unspecific bounding of antibodies. After this "blocking" step the cells were stained with an antibody-cocktail consisting of SiglecF-PE, F4/80-FITC, GR1-APCCy7, CD11b-PE-Cy7, CD3-PerCP and CD4-APC.

The eosinophils were defined as small cells with considerable sideward light scattering properties, indicative of complex granules. Eosinophils have been described to express high levels of SiglecF. This marker is a sialic acid-binding Ig superfamily receptor (Zhang et al 2007) and shall be deemed to be largely eosin-specific, but some reports suggest that alveolar macrophages during inflammatory process in the lungs can also express this marker. To separate eosinophils from macrophages, the cells were gated on SiglecF vs. F4/80, because F4/80 is described as a very specific marker for macrophages (Austyn et al., 1981). Large cells with high granularity that express F4/80 at a high level can be identified as macrophages (or monocytes); cells with high SiglecF expression were defined as eosinophils. Monocytes were identified as F4/80 expressing cells, which are also GR1 and CD11b positive and smaller as macrophages with a lower SSC. Neutrophils have almost identical size as eosinophils but are less granular and express only GR1 and no SinglecF on their surface. T helper cells are small and show low granularity; these cells can be identified by gating on CD4 vs. CD3, two markers of CD4 positive T cells.

\subsubsection{Establishment of GC-therapy of AAI and mouse strain characteristics}

The histological observation concerning the increased influx of inflammatory cells into the lungs was confirmed by analysing cell numbers in the BAL from the wild type BALB/c and C57BL/6 mice. The healthy mice in both cases had only about 100000 cells while in the lavage of asthmatic mice there were about $1.5 \times 10^{6}$ inflammatory cells after the induction of the allergic response (Figure 14). The peak of inflammation was observed about 40 hours 
after the last challenge with antigen. Based on this observation the analysis of BAL cell numbers and cell composition were always done at this time point.

Although there are several reports, that the C57BL/6 mouse strain develops a less strong $\mathrm{T}_{\mathrm{H}} 2$ response compared to $\mathrm{BALB} / \mathrm{c}$ mice, the analysis of inflammation in our mouse model of asthma showed no significant difference between these two genotypes (Figure 14).

In order to investigate the mechanism of GC-action in the treatment of AAI, first experiments were done using wild type animals. The goal of this study was to analyse the normal response to the therapy with Dexamethasone and to establish the treatment protocol. Treatment with $10 \mathrm{mg}$ per $1 \mathrm{~kg}$ body weight was proven to be successful and strong enough to significantly reduce inflammation in AAI-animals. The treatment of mice of both genetic backgrounds led to a strong reduction of the cell number in the BAL. While the BAL fluid of AAI-animals contained more than $1.5 \times 10^{6}$ infiltrating cells, the BAL of GC-treated group showed largely reduced cell numbers.

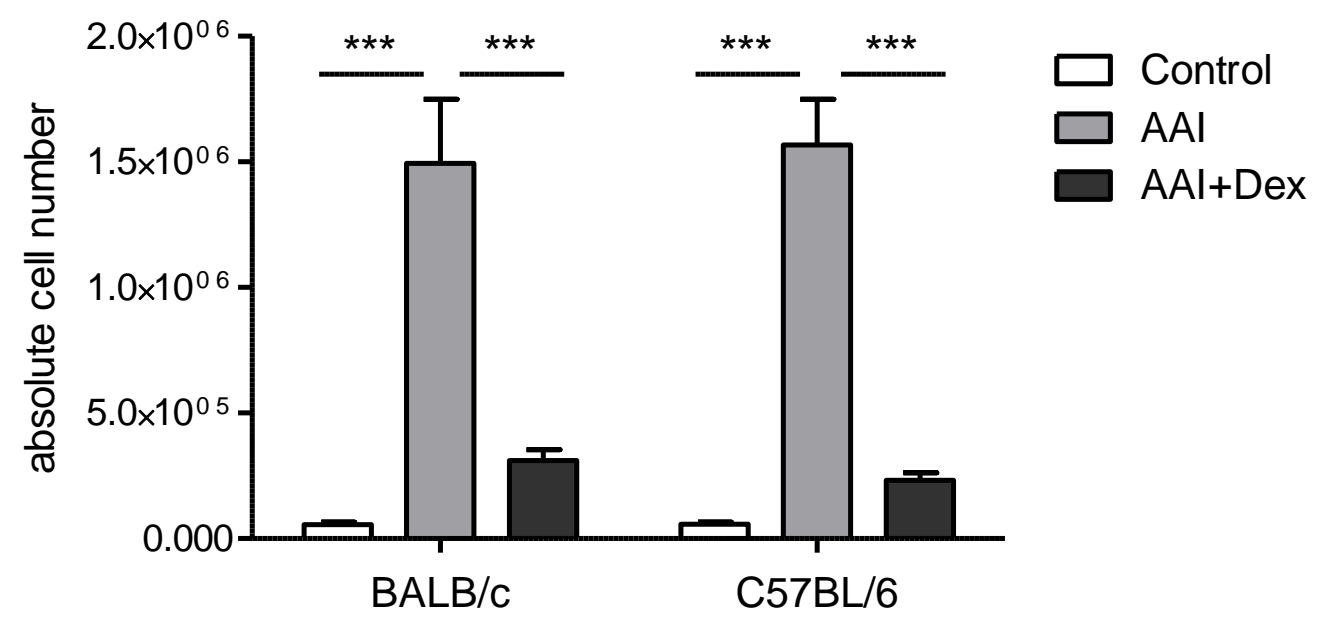

Figure 14: BAL cell number of BALB/c and C57BL/6 mice. AAI was induced by repeated Ovaimmunisation and challenge. To analyse the effect of GC-therapy one part of the AAI mice was treated with Dex $(10 \mathrm{mg} / \mathrm{kg})$. To compare infiltration into the lungs of healthy, AAI and Dex-treated AAI mice on a BALB/c or C57BL/6 background, the BAL was isolated $40 \mathrm{hrs}$ after the last Ovachallenge and the absolute cell number of BAL samples was determined using a Neubauer counting chamber. The diagram represents the mean absolute cell numbers +/- SEM (BALB/c: control $n=15$; AAI n=17; AAI+Dex n=16; C57BL/6: control n=17; AAI n=18; AAI+Dex n=21).

The cell type composition was analysed by FACS as described above. The data demonstrated that the increased number of BAL cells in AAI mice directly correlated with a strong eosinophilia in the lungs. The largest fraction of BAL cells (more than $70 \%$ ) belonged to the eosinophil population after induction of asthma-like reaction while the 
population of macrophages, neutrophils and T cells together make up less than $30 \%$ of all BAL cells (Figure 15).

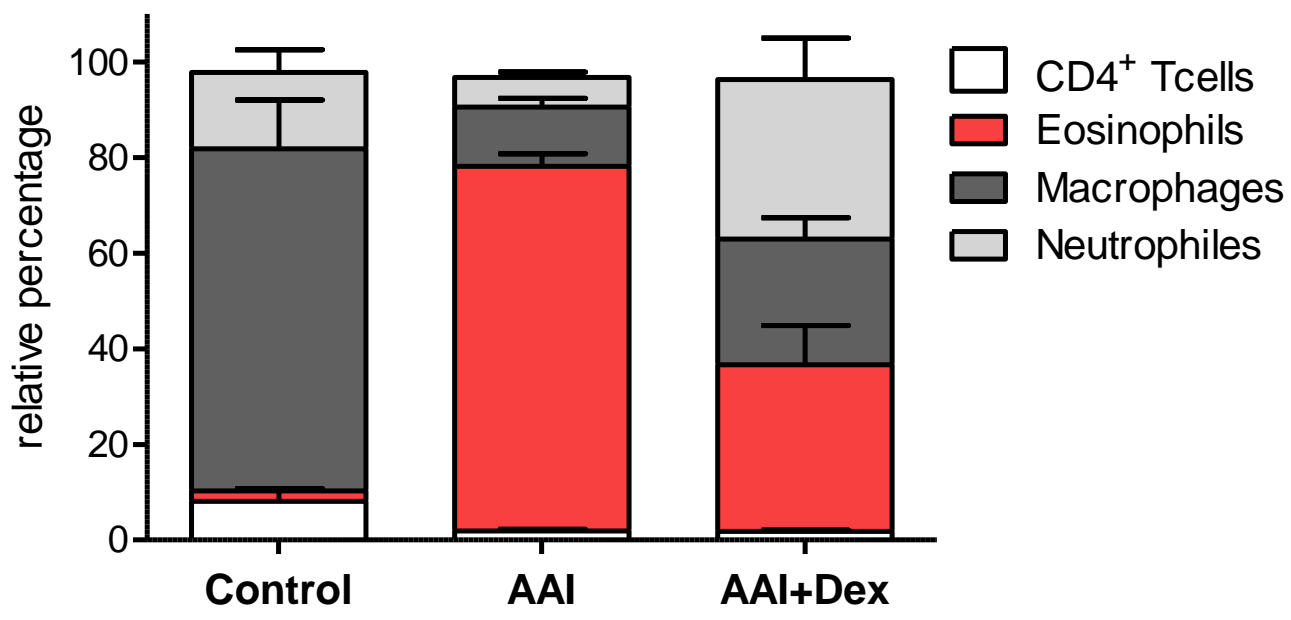

Figure 15: Analysis of the percentages of different BAL subpopulations in healthy (control), AAI and Dex-treated AAI wild type (BALB/c) mice. AAI was induced as described in Figure 10. The isolated BAL was analyzed by FACS to investigate the relative percentages of $\mathrm{T}$ helper cells, eosinophils, macrophages and neutrophils. The data represent the mean of the relative percentages with +/- SEM for 11 healthy, AAI and Dex-treated AAI mice.

The treatment of wild type mice with Dexamethasone reduced the fraction of eosinophils to less than $40 \%$. The largest cell population in the BAL of healthy mice were macrophages, which make up about $70 \%$ of all cells. The fraction of macrophages in Dexamethasonetreated AAI mice increases in comparison to AAI mice and constitutes about one third of the BAL cells. Dex-treatment of AAI mice does not reduce the percent of neutrophils in the BAL: compared to the lung infiltrate of AAI mice the neutrophils in the Dexamethasonetreated mice make up about one third of the BAL cells.

To further analyse the cellular infiltration and GC-effects on the BAL cell composition, the absolute cell numbers of different BAL subpopulations were determined in BALB/c and C57BL/6 mice. The results demonstrated that AAI could be successfully induced in both mice strains with a very similar BAL cell composition and cell numbers. The largest cell population in the BAL of AAI mice consisted of eosinophils (with more than 1 x $10^{6}$ cells) while the BAL of normal healthy animal contains only a few hundred eosinophils. 


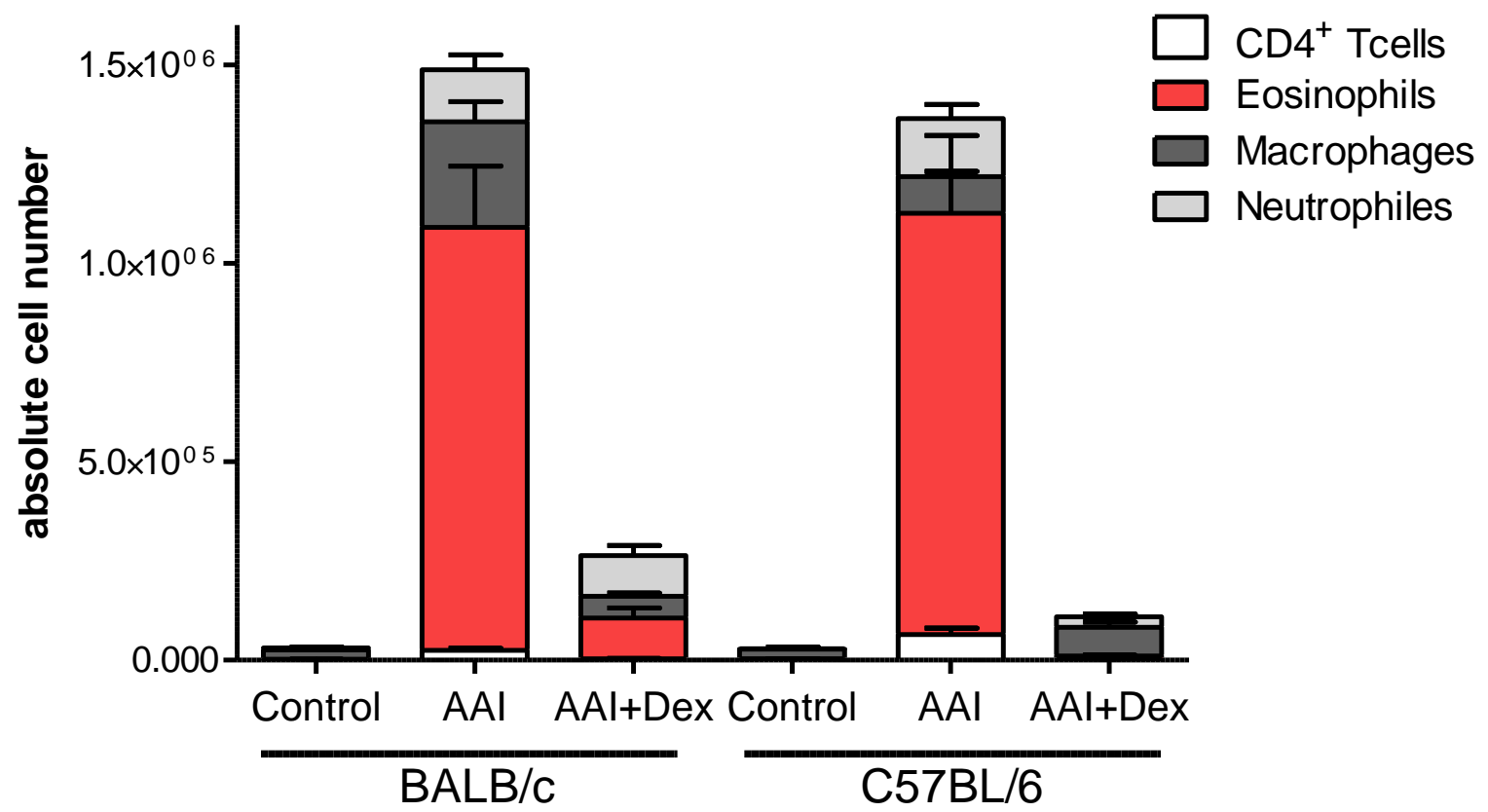

Figure 16: BAL cell composition in BALB/c and C57BL/6 mice. Based on the percentage of the subpopulations, the absolute cell number of each subpopulation could be calculated. The BAL samples were collected from healthy (Control), AAI and Dex-treated AAI mice of BALB/c and C57BL/6 genotype as described before in Figure 14. The diagrams represent the mean of absolute cell number +/- SEM (BALBc: Control $n=15$, AAI $n=15$, AAI+Dex $n=14$; C57BL/6: Control $n=13$, AAI $n=15$, AAI+Dex $n=16$ )

The remaining of the BAL cell fraction from AAI mice of both strains consisted of macrophages and neutrophils. The Dexamethasone treatment successfully reduced the cell number of the eosinophils in both mouse strains (Figure 16). The cell number of macrophages and neutrophiles remained mostly unchanged after Dexamethasone treatment. This analysis and the observed effects of Dexamethasone on the number of inflammatory cells in the infiltrate demonstrated that both wild type mouse strains were treatable with Dexamethasone doses and showed a very similar response to the therapy.

Another important marker of the allergic response is an increased antigen-specific immunoglobulin production with class switch to $\operatorname{IgE}$ and $\operatorname{IgG}$ isotypes. Although there are asthmatic patients without increased immunoglobulin levels, these mediators play a crucial role in developing an inflammatory reaction. In a healthy non-atopic organism the level of $\operatorname{IgE}$ and $\operatorname{IgG}$ are very low and nearly undetectable, while in the serum of an allergic person the level of these immunoglobin isotypes is hundred times increased. This observation was confirmed in our mouse model of an asthma-like reaction. The animals immunised and 
sensitised to Ova showed a strong increase in the level of Ova-specific IgE, IgG2a and IgG1 in the serum that was demonstrated by an antigen-specific ELISA (Figure 17).
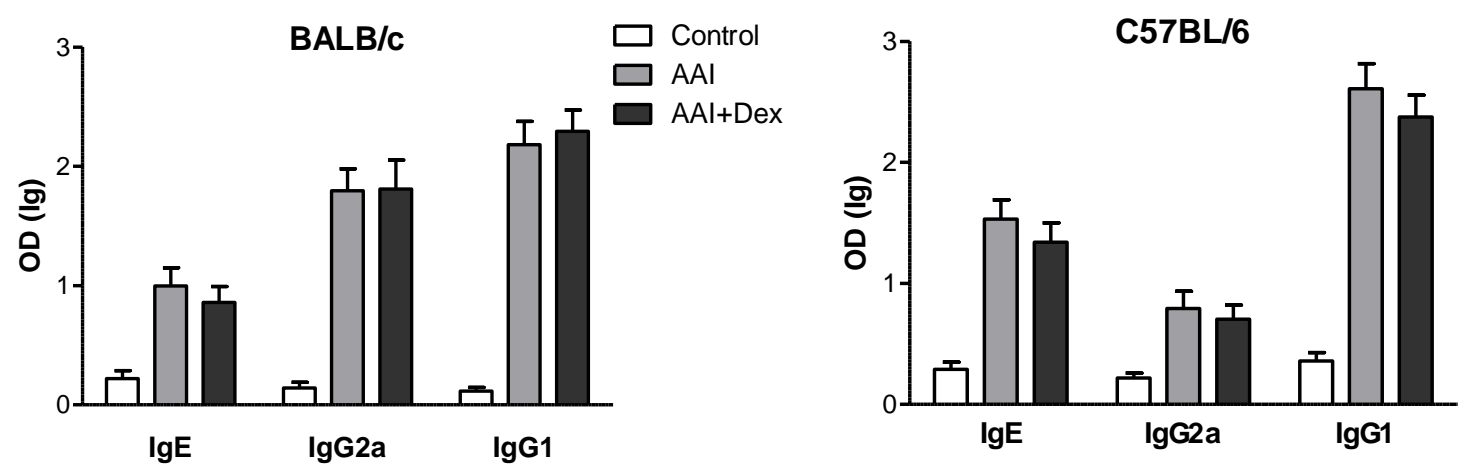

Figure 17: Antigen-specific immunoglobulin production in BALB/c and C57BL6 wild type mice. Serum was collected from each animal and the Ig level was measured using an Ova-specific Ig-ELISA. For Ig detection different dilutions of the serum samples were used:

BALB/c: IgE 1:1000, IgG2a 1:10 000; IgG1 1:100 000; C57BL/6: IgE 1:100; IgG2a 1:1000; IgG1 1:100 000. The data represent the mean OD +/- SEM (BALB/c: Control n=17, AAI n=18, AAI+Dex $\mathrm{n}=19$; C57BL/6: Control n=16, AAI n=18, AAI+Dex n=21)

High levels of $\operatorname{IgE}, \operatorname{IgG} 2 \mathrm{a}$ and $\mathrm{IgG} 1$ were detected in the serum of AAI mice of BALB/c and C57BL/6 mice. Both mouse strains produced a very high level of Ovalbumin-specific IgG1 while the production of IgE and IgG2a was much less. While the serum of BALB/c mice could be diluted 1:1000 for the IgE detection the samples of C57BL/6 mice had to be diluted 1:100. Also the preparation of samples for detection of $\operatorname{IgG} 2 \mathrm{a}$ was made with different dilution grade because the serum of BALB/c mice could be diluted 10-fold more than that of C57BL/6 mice. The IgG1 level was comparable between both mouse strains. Treatment of AAI mice with Dexamethasone did not lead to reduced production of immunoglobulins.

\subsubsection{Establishment of NIR imaging of Asthma in the mouse model of AAI}

\subsubsection{NIR Imaging with ProSense680}

To investigate the inflammation in the lungs, NIR Imaging was used as a non-invasive method. First of all, wild type mice under different conditions (control, AAI and Dexamethasone-treated AAI mice) were injected with the NIR imaging agent ProSense680. ProSense680 allows detecting activated eosinophils, macrophages, neutrophils and other inflammated cells in the lungs due to its fluorescence only in the activated stage after cleavage by a family of lysosomal cathepsin proteases. 
Corresponding to the basal state of ProSense680 the fluorescent signal from the lung region of control healthy mice was very low. In contrast, the fluorescent signal from the lung region of mice with AAI strongly increased 24 hours after the last challenge with Ova.

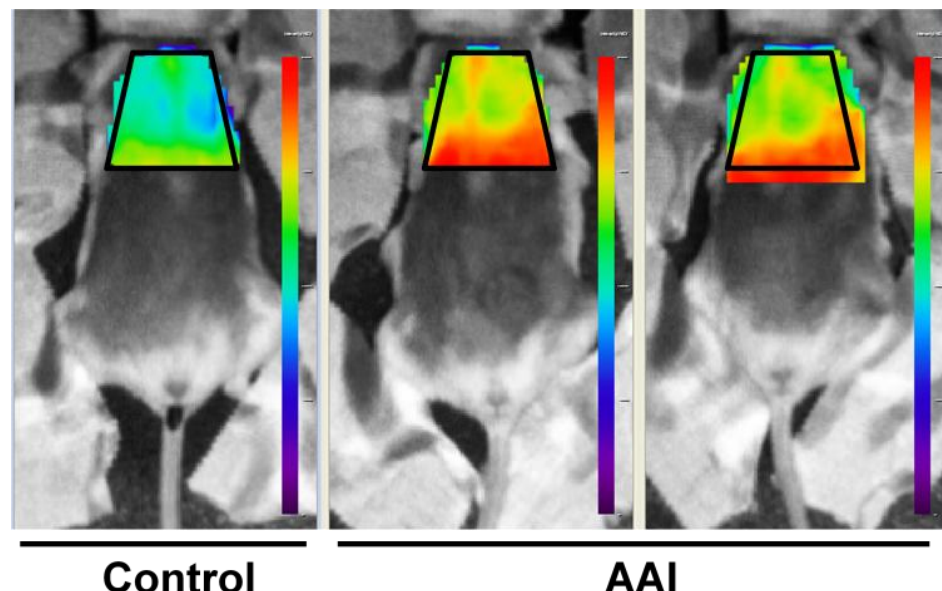

AAl

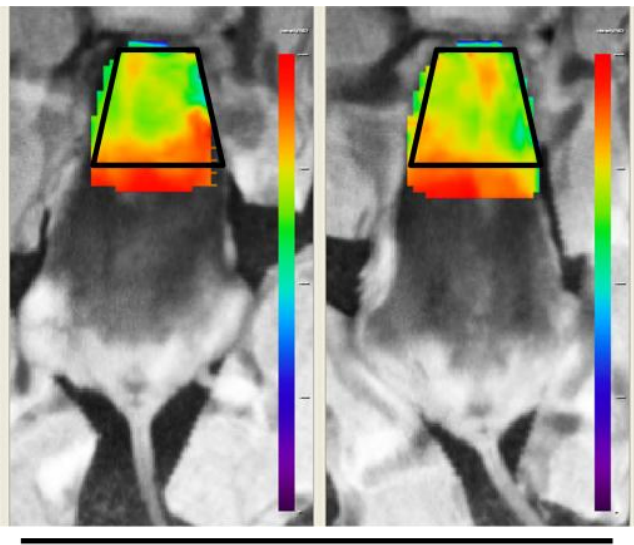

AAl+Dex

Figure 18: NIR in vivo imaging with ProSense680 as an imaging agent. AAI was induced by repeated sensitisation and challenge with Ova (with or without Dexamethasone treatment) as described before in Figure 10. The fluorescence level was measured $24 \mathrm{hrs}$ after the last challenge with Ova (and 20 hrs after ProSense680 dye injection). The acquired images were analysed with OptiView software and demonstrate the fluorescence level in the lung region of healthy (control), $\mathrm{AAI}$ and Dexamethasone-treated AAI mice measured in vivo (control $\mathrm{n}=1$; AAI $\mathrm{n}=2$, AAI+Dex $\mathrm{n}=2$ ).

It could also be demonstrated that while the fluorescence level in AAI mice strongly increased compared to healthy mice there was no significant reduction of the fluorescence signal after Dex-treatment. This analysis was repeated every 12 hours for two days, but the correlation between the treatment and fluorescence signal from the lung region remained unchanged (data not shown). 
The observation, that there was no significant difference between the fluorescence intensity from AAI and GC-treated lungs could was confirmed by quantification of the signal (Figure 19). The calculation was done automatically using OptiView-2 02-00 software (ART). Thereby the counts of the fluorescent pixels were normalized to the laser power and the basal fluorescence (data of prescan).

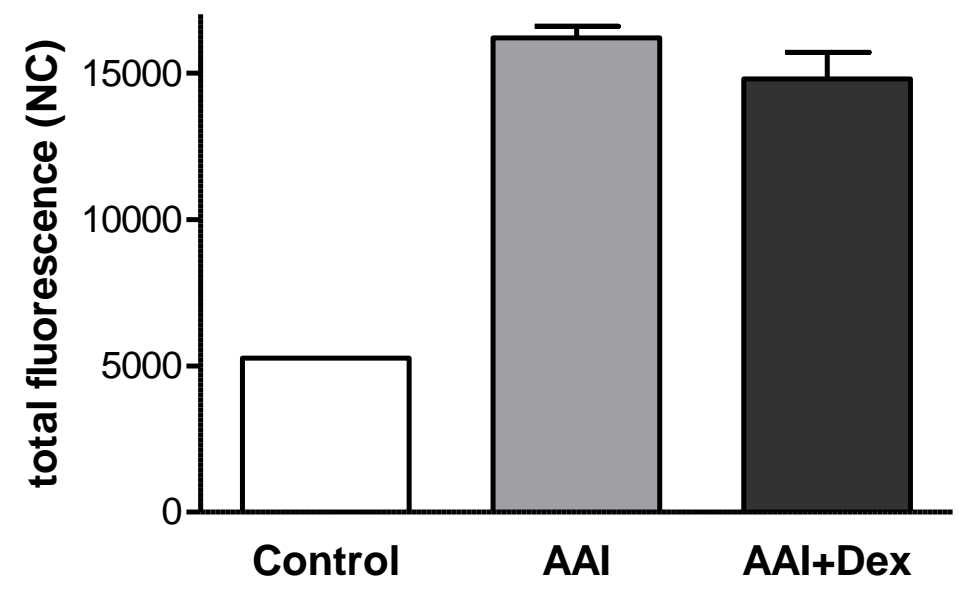

Figure 19: Quantification of total fluorescence counts of activated ProSense680 from the lung region of control $(n=1)$, AAI $(n=2)$ and Dex-treated AAI $(n=2)$ wt BALB/c mice. The fluorescence level was measured $24 \mathrm{hrs}$ after the last challenge of the mice with Ova (20 hrs after ProSense680 dye injection). AAI was induced as described before in Figure 10. The data represent the mean of total counts (normalized to the basal fluorescence from the lung region and the used laser power).

\subsubsection{NIR Imaging and Immunohistochemistry with Anti-SiglecF}

Lung infiltration by eosinophils and the effects of GC-treatment were also analysed using NIR Imaging with the eosinophil-specific marker SiglecF labelled with Alexa-Fluor750 as well as immunohistochemistry with an anti-SiglecF antibody.

With the help of anti-SiglecF staining of lung sections it could be shown that SiglecF binds very specifically to the eosinophils in AAI mouse lungs. While the infiltrating eosinophils were located around the conducting small and large airways in the mice with an allergic reaction, the macrophages in the lungs of control mice remained unstained. 


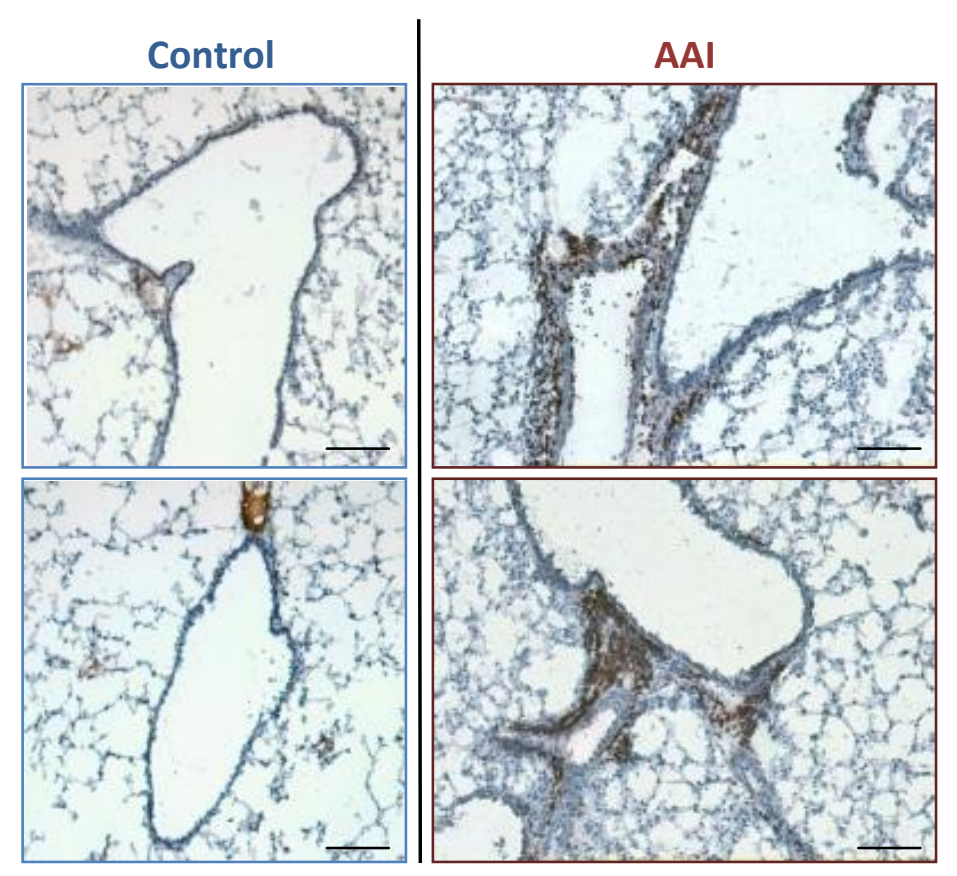

Figure 20: anti-SiglecF staining of lung sections from healthy (control) and AAI wt BALB/c mice. For the histological analysis of eosinophils $10 \mu \mathrm{m}$ cryo-sections of lung tissue were cut and stained with an anti-SiglecF antibody. The left pictures represent the lung parenchyma and the conducting airways (at 10x magnification) of healthy control mice. The right pictures show typical inflammatory lung tissues (at 10x magnification) and bronchioles surrounded by eosinophils of mice with AAI. The scale bars in the lower right corner of all images equal $100 \mu \mathrm{m}$.

To analyse the eosinophilia in the lungs after the development of an asthma-like reaction different time points were used for the imaging with the anti-SiglecF-AlexaFluor750 antibody. The peak of the fluorescence signal from the lung region was detected 24 hours after the last challenge (20 hours after the anti-SiglecF injection).

Compared to the data obtained with ProSense680, where Dexamethasone had no effect on the fluorescence intensity of the lungs, the imaging data with the anti-SiglecF agent revealed a reduction of the fluorescence in vivo. Because of possible unspecific fluorescence signals from the liver region (under the lungs), an ex vivo imaging was done with the dissected lungs. 
A
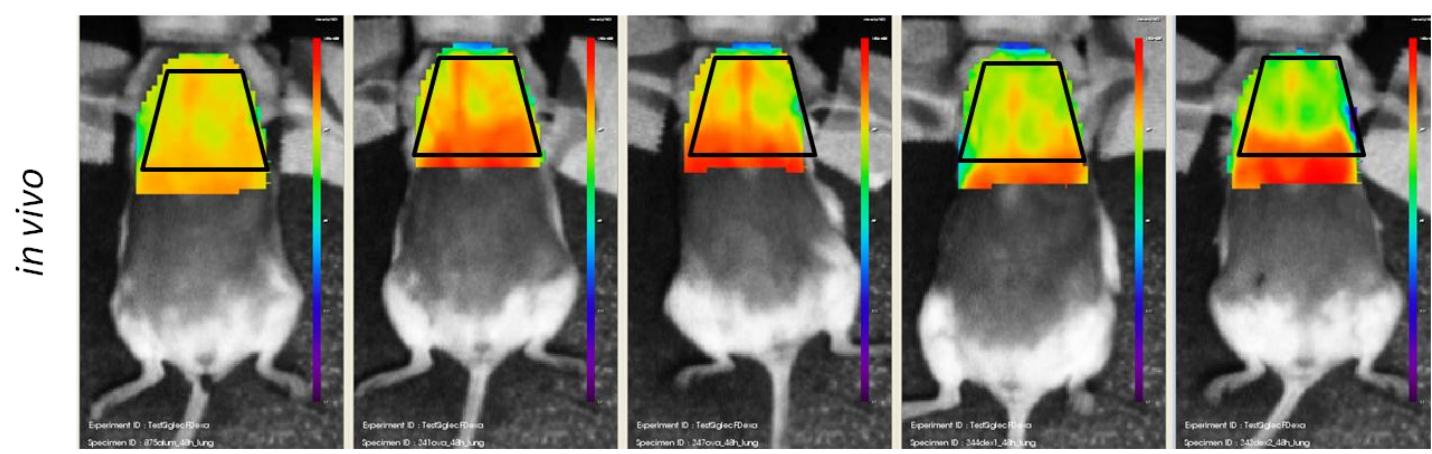

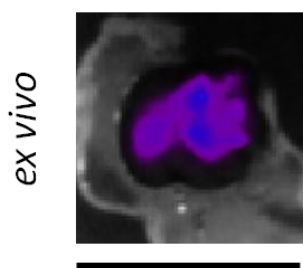

Control

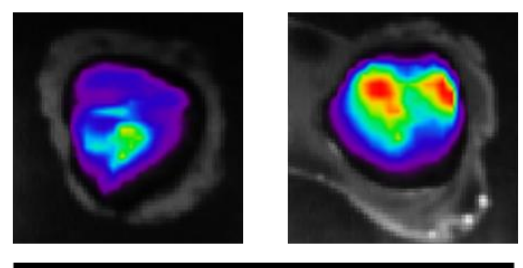

AAI

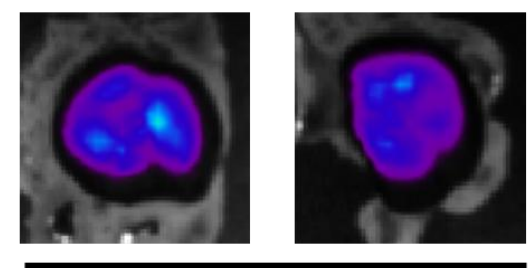

AAI+Dex
B

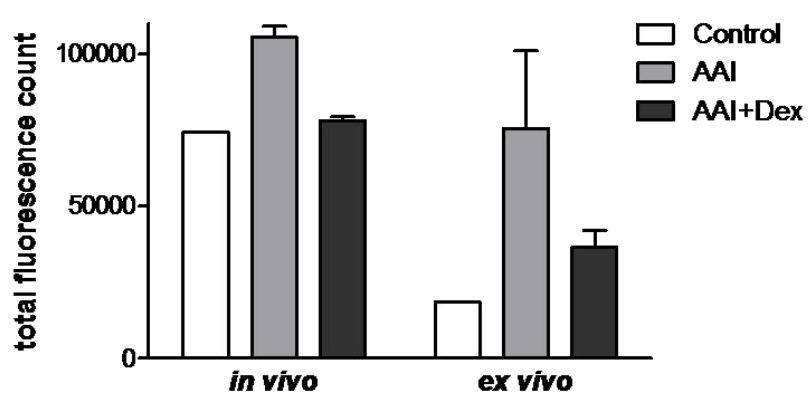

C

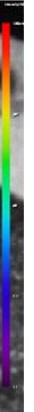




\subsection{Identification of target cell populations of GCs by analyzing therapy efficiency in different GR-mutant mouse stains}

To identify essential target cells of GCs in this work were used cell-type specific GRdeficient mice which lack the GR in myeloid cells (GR ${ }^{\text {lysM }}$, Tuckermann JP, et al. (2007), T cells (GR ${ }^{\text {lck }}$, Baumann S, et al. (2005)), DCs (GR ${ }^{\mathrm{CD} 11 \mathrm{c}}$, Caton ML, Smith-Raska MR, Reizis B (2007)) or B cells (GR ${ }^{\text {CD19 }}$, Rickert RC, Roes J, Rajewsky K (1997). These mice were generated by crossing $\mathrm{GR}^{\text {flox }}$ mice (used in these experiments as wild type control mice) with lysMCre, lckCre, CD11CCre or CD19Cre mice respectively and back-crossed to either the C57BL/6 or BALB/c background.

\subsubsection{Analysis of $G^{\text {lysM }}$ mice on BALB/c and C57BL/6 background}

The myeloid cell lineage plays an important role in the pathogenesis of many inflammatory and autoimmune diseases. These cells are located in the lung tissue (alveolar macrophages) but are also part of the host defense mechanisms and the largest leukocyte population in the healthy lungs. On the other hand macrophages play an important role for the improvement of lung functions and can be seen as important targets for GC-therapy.To investigate GC effects on myeloid cells during therapy of an asthmatic reaction mice were used which lack the GR in cells of the myeloid lineage. To compare the effects of the treatment and its efficiency wild type and $\mathrm{GR}^{\text {lysM }}$ mice on both BALB/c and C57BL/6 backgrounds were employed.

\subsubsection{Analysis of GR-expression in eosinophils in the BAL of GR ${ }^{\mathrm{lysM}}$ mice}

$\mathrm{GR}^{\text {lysM }}$ mice were reported to lack the GR in myeloid cells, such as monocytes, macrophages, neutrophils, and osteoclasts (Tuckermann et al., 2007). In order to investigate GR-expression in eosinophils, they were isolated from the BAL of AAI mice by FACS sorting. The mRNA expression level of the GR was determined by RT-qPCR.

The data from this experiment demonstrated that in comparison to wild type mice on a $\mathrm{BALB} / \mathrm{c}$ or $\mathrm{C} 57 \mathrm{BL} / 6$ background GR-expression in $\mathrm{GR}^{\text {lysM }}$-eosinophils was partially reduced. Additionally, differences could be observed in eosinophils from $\mathrm{GR}^{\text {lysM }}$ mice on a BALB/c or C57BL/6 background. While $\mathrm{GR}^{\text {lysM}} / \mathrm{BALB} / \mathrm{c}-\mathrm{eosinophils} \mathrm{showed} \mathrm{only} \mathrm{about}$ $20 \%$ of normal GR-expression, the GR ${ }^{1 y s M} / \mathrm{C} 57 \mathrm{BL} / 6$-eosinophiles had more than $70 \%$ of GR-expression. 


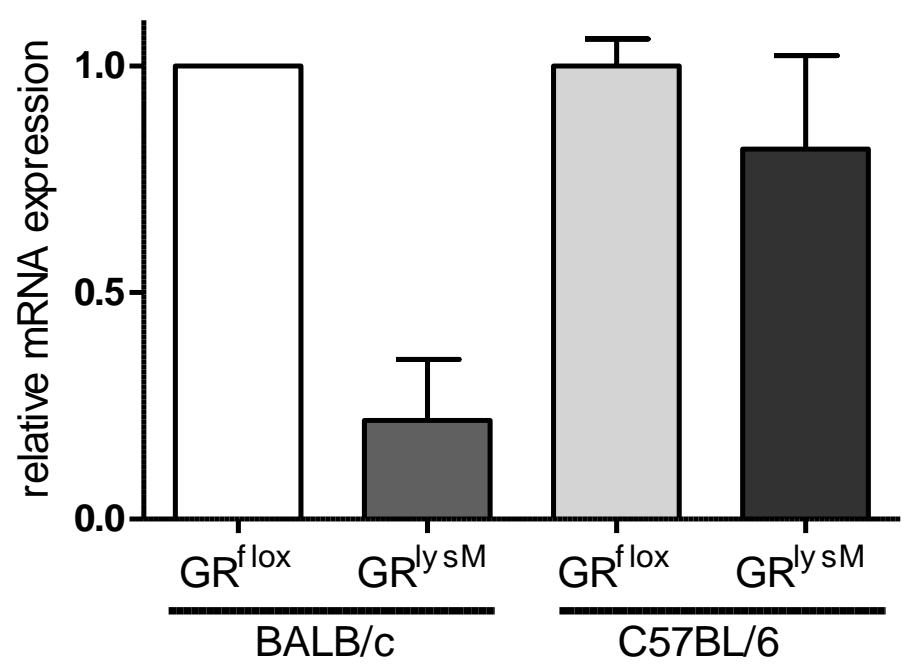

Figure 22: GR-expression analysis in eosinophils from $\mathbf{G R}^{\text {flox }}$ and $\mathbf{G R}^{\mathrm{lysM}}$ mice on a BALB/c and C57BL/6 background. Eosinophils were isolated from the BAL of $\mathrm{GR}^{\mathrm{lysM}}$ mice (on a BALB/c and C57BL/6 background) and GR $^{\text {flox }}$ mice by FACS sorting. The mRNA expression of the GR was measured by RT-qPCR and normalized to HPRT expression. The GR mRNA expression of GR $^{\text {flox }}$ mice was set equal to 1 . The data represent the mean relative expression +/- SEM (BALBc: GR $^{\text {flox }}$ $\mathrm{n}=1 ; \mathrm{GR}^{\text {lysM }} \mathrm{n}=3 ; \mathrm{C} 57 \mathrm{BL} / 6 \mathrm{GR}^{\text {flox }} \mathrm{n}=4 ; \mathrm{GR}^{\text {lysM }} \mathrm{n}=9$ )

Based on this observation, $\mathrm{GR}^{\text {lysM }}$ mice are useful for the evaluation of the role of macrophages and monocyte in asthma but only partially for the analysis of the role of eosinophils in this process. 


\subsubsection{Analysis of AAI and treatability of $G^{\text {lysM }}$ mice on a BALB/c background with GCs}

\subsubsection{BAL analysis}

Determination of BAL cell numbers in $\mathrm{GR}^{\text {flox }}$ and $\mathrm{GR}^{\text {lysM }}$ mice suggested a similar reaction to AAI induction after the Ova-sensitization in both strains.

A

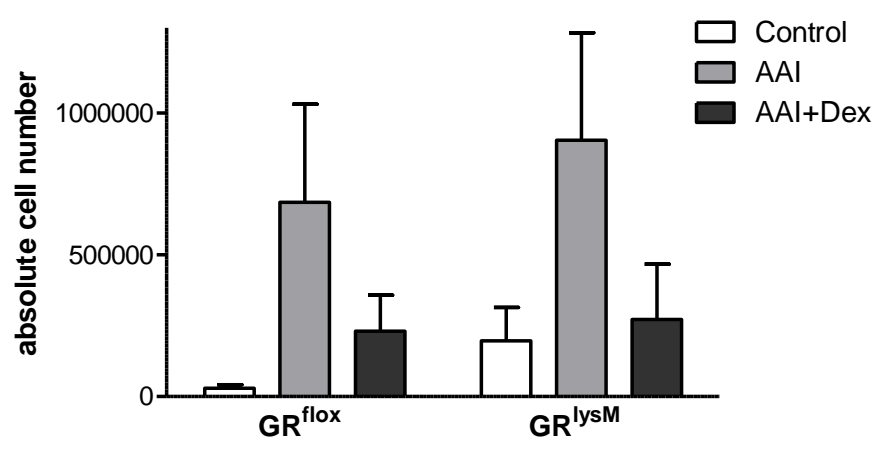

B

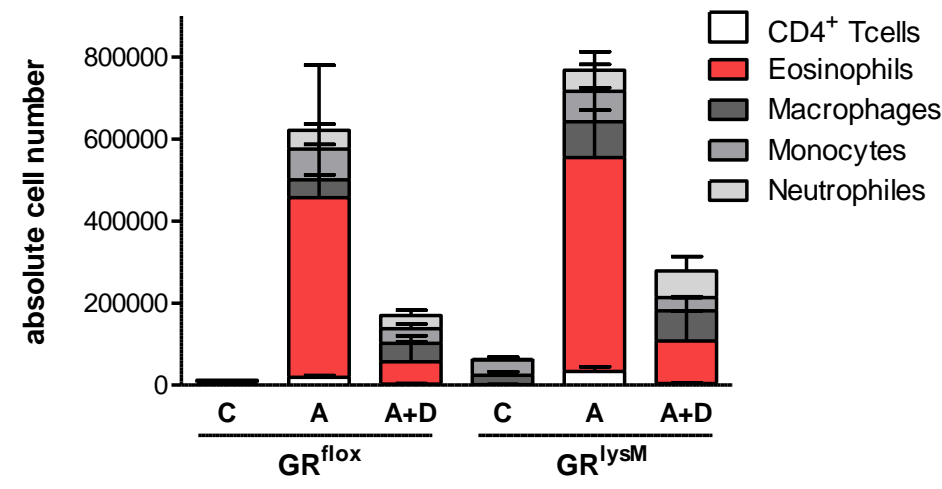

Figure 23: BAL cell numbers (A) and BAL cell composition $(B)$ in $G^{1 y s M}$ mice on a BALB/c background. (A) The BAL of healthy (control, C), AAI (A) and Dex-treated (AAI+Dex; A+D) mice was quantified concerning the absolute cell number with the help of Neubauer cell count chamber. (B) To investigate BAL cell subpopulations the isolated BAL samples were analyzed by FACS. The relative percentages and absolute cell numbers of the BAL were used for the calculation of the absolute cell numbers of the subpopulation. The data represent the mean absolute cell number $+/-$ SEM $\left(\mathrm{GR}^{\text {flox }}\right.$ : control $n=2$. AAI $n=5 ;$ AAI + Dex $n=3 ; \mathrm{GR}^{\text {lysM }}$ control n=3; AAI n=4; AAI+Dex n=4)

The BAL cell composition revealed a strong eosinophilia in the lungs of wild type and $\mathrm{GR}^{\text {lysM }}$ mice that could be significantly reduced by GC-therapy. In the BAL of GC-treated animals the eosinophil influx into the lungs could be successfully inhibited- 


\subsubsection{Proliferation and priming in $G R^{\text {flox }}$ and $G R^{\text {lysM }}$ mice}

GR-depletion in myeloid cells could cause altered proliferation and priming of antigenspecific $\mathrm{T}$ and $\mathrm{B}$ cells. To test this possibility it was interesting to analyze the proliferation of splenocytes from these mutant mice.
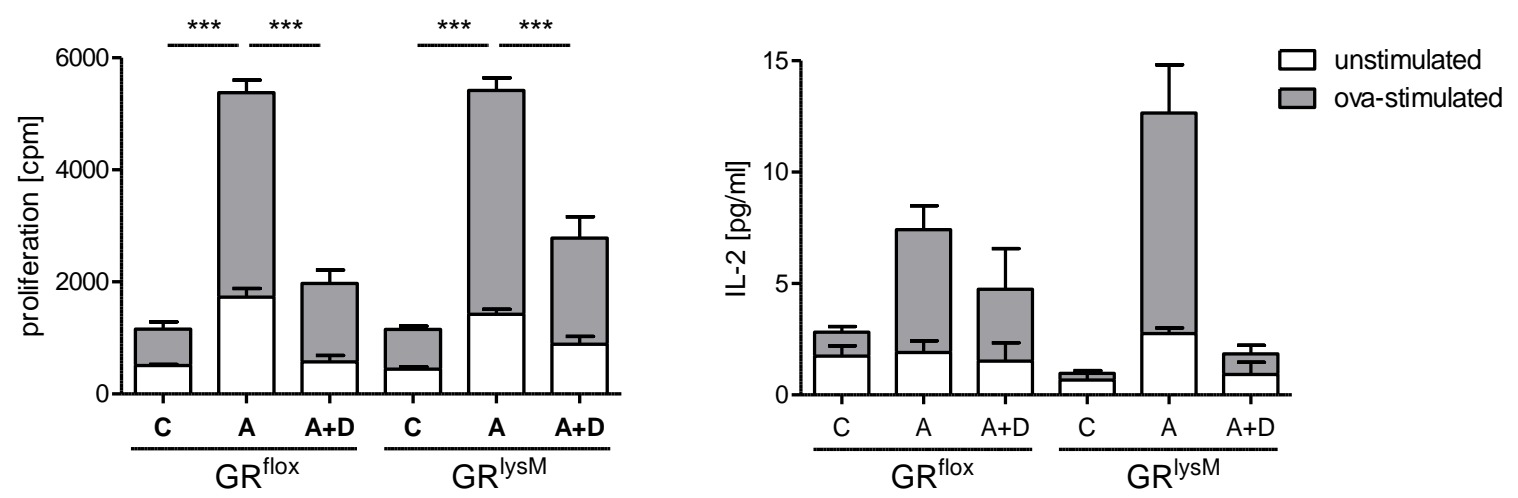

Figure 24: Proliferation and IL-2 secretion of splenocytes from $\mathbf{G R}^{\text {flox }}$ and GR $^{\text {lysM }}$ mice after antigen-specific restimulation ex vivo. Proliferation of splenocytes from healthy control (C), AAI (A) and Dex-treated AAI (A+D) mice was determined after $72 \mathrm{hrs}$ incubation of 300000 splenocytes with antigen-specific restimulation (Ova), compared to the basal proliferation (unstimulated cells). $50 \mu 1$ of supernatant were collected and the IL-2 release was determined via ELISA. The cells were incubated for another $16 \mathrm{hrs}$ in medium containing ${ }^{3} \mathrm{H}$-thymidine and were analysed for proliferation activity using a ${ }^{3} \mathrm{H}$-thymidine incorporation assay. The results show the mean proliferation (cpm) or IL-2 concentration $[\mathrm{pg} / \mathrm{ml}]+/$ - SEM of triplicate measurements $\left(\mathrm{GR}^{\text {flox }}\right.$ : control $\mathrm{n}=1$; AAI $\mathrm{n}=4$, $\mathrm{AAI}+\operatorname{Dex} \mathrm{n}=2 ; \mathrm{GR}^{\mathrm{lys} M}$ : control $\mathrm{n}=2 ; \mathrm{AAI} \mathrm{n}=3 ; \mathrm{AAI}+\operatorname{Dex} \mathrm{n}=2$ )

The proliferation rate of splenocytes from asthmatic $\mathrm{GR}^{\text {flox }}$ and $\mathrm{GR}^{\text {lysM }}$ mice was increased after antigen-specific restimulation ex vivo. The splenocytes from both $\mathrm{GR}^{\text {flox }}$ and $\mathrm{GR}^{\text {lysM }}$ mice showed nearly the same response to GC-therapy and their proliferation activity was suppressed. The same effects of GC-therapy could be seen on IL-2 secretion. Compared to the IL-2 concentration in supernatants of splenocytes from asthmatic mice the concentration of this cytokine in samples of GC-treated animals was several fold decreased.

Because the IL-4 and IFNy concentration in the supernatants of the examined mice was very low it was difficult to determine the correlation between the treatment and IL-4 or IFNy secretion. In principle, restimulation of $\mathrm{GR}^{\text {flox }}$ and $\mathrm{GR}^{\text {lysM }}$ splenocytes ex vivo led to an increased secretion of IL-4 and IFNy in samples of asthmatic mice's on a BALB/c background. The therapy with Dexamethasone led to stronger production of IL-4 and a reduction of IFNy production in both genotypes (data not shown). 


\subsubsection{Immunoglobulin secretion and isotype class switching}

Secretion of Ova-specific immunoglobulins in $\mathrm{GR}^{\text {lysM }}$ mice was not impaired by the GR-knockout in myeloid cells. As the Ig-analysis data could demonstrate the Ig class switch and production was very similar between AAI and also Dex-treated GR flox and GR ${ }^{\text {lysM }}$ mutant mice on BALB/c background.
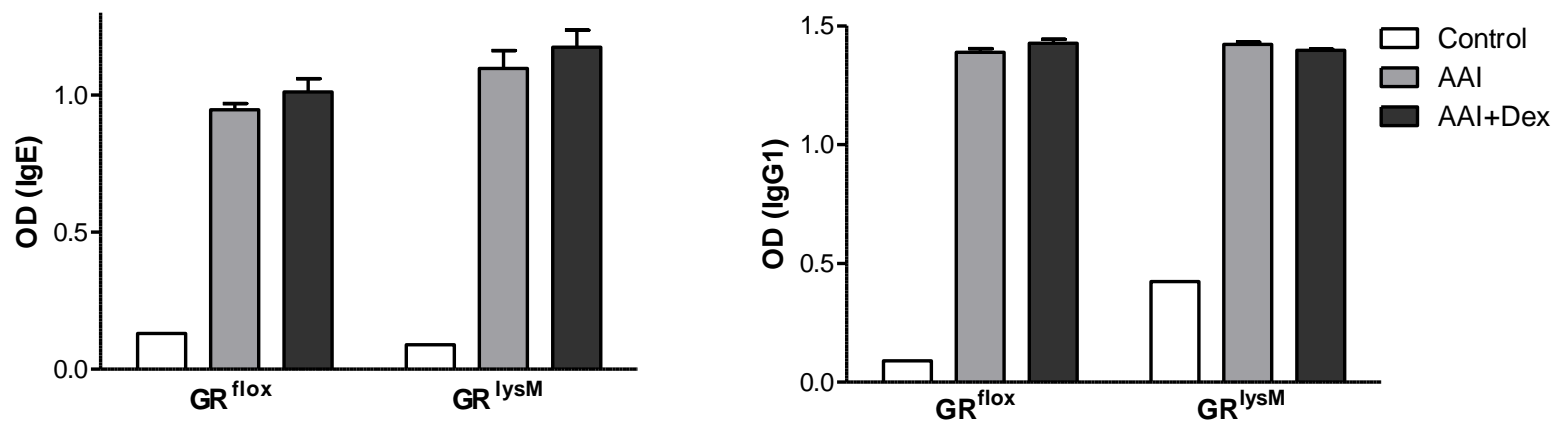

Figure 25: Immunoglobulin isotype production in serum samples of $G^{\mathrm{lysM}}$ and $\mathbf{G R}^{\mathrm{flox}}$ mice on a BALB/c background. The Ig secretion and isotype switch were analyzed using an Ova-specific IgELISA of serum from healthy (control), AAI and Dexamethasone-treated (AAI+Dex) mice. The dilution of the serum was dependent on the Ig isotype and the genetic background. GR ${ }^{\text {lysM }} / \mathrm{BALB} / \mathrm{c}$ : IgE 1:100, IgG1 1:100000. The data show the mean OD +/- SEM $\left(\mathrm{GR}^{\text {flox }}\right.$ : control $n=1$; AAI $n=4$; AAI+Dex n=2; GR ${ }^{\text {lysM }}$; control n=1; AAI n=3; AAI+Dex n=2)

The serum samples used for the detection of Ig isotypes were diluted to different degrees. For the IgE measurement the samples of $\mathrm{GR}^{\mathrm{lysM}} / \mathrm{BALB} / \mathrm{c}$ were diluted $1: 100$. The $\mathrm{IgG} 1$ isotype was present in serum of Ova-sensitized mice at the highest concentration and could therefore be diluted 1:100 000 for $\mathrm{GR}^{\mathrm{lysM}} / \mathrm{BALB} / \mathrm{c}$ mice.

\subsubsection{Analysis of AAI induction and the efficiency of GC-therapy inGR ${ }^{\text {lck }}, \mathbf{G R}^{\text {lysMlck }}$ and $\mathrm{GR}^{\mathrm{lysM}}$ mice on a C57BL/6 background}

Next GR ${ }^{1 \mathrm{lck}}, \mathrm{GR}^{\text {lysMlck }}$ and $\mathrm{GR}^{\text {lysM }}$ mice on a C57BL/6 background were analyzed. GR ${ }^{\text {lck }}$ mice lack the GR on all $\mathrm{T}$ cell populations and can be used for the investigation of direct GCeffects on $\mathrm{T}$ cells during GC-therapy of asthmatic reactions. The $\mathrm{GR}^{\mathrm{lysM}}$ mice (described before) lack GR expression in myeloid cells. The $\mathrm{GR}^{\text {lysMlck }}$ mice are double knockout mice in which the GR is depleted not only on T cells but also in the myeloid lineage. This double knockout provides the possibility to investigate not only the role of $\mathrm{T}$ cells and myeloid cells for the GC-treatment separately but also the role of these cells in combination. 


\subsubsection{BAL analysis}

First of all the cell number of the BAL from $\mathrm{GR}^{\mathrm{lck}}, \mathrm{GR}^{\text {lysM }}$ and $\mathrm{GR}^{\text {lysMlck }}$ mice was determined. The mutant strains developed a strong inflammatory reaction in the lungs with large amount of infiltrating cells. This cellular infiltration was comparable between $\mathrm{GR}^{\mathrm{lck}}$, $\mathrm{GR}^{\text {lysM }}$ and $\mathrm{GR}^{\text {lysMlck }}$ mice and reached more than $1.5 \times 10^{6}$ cells.
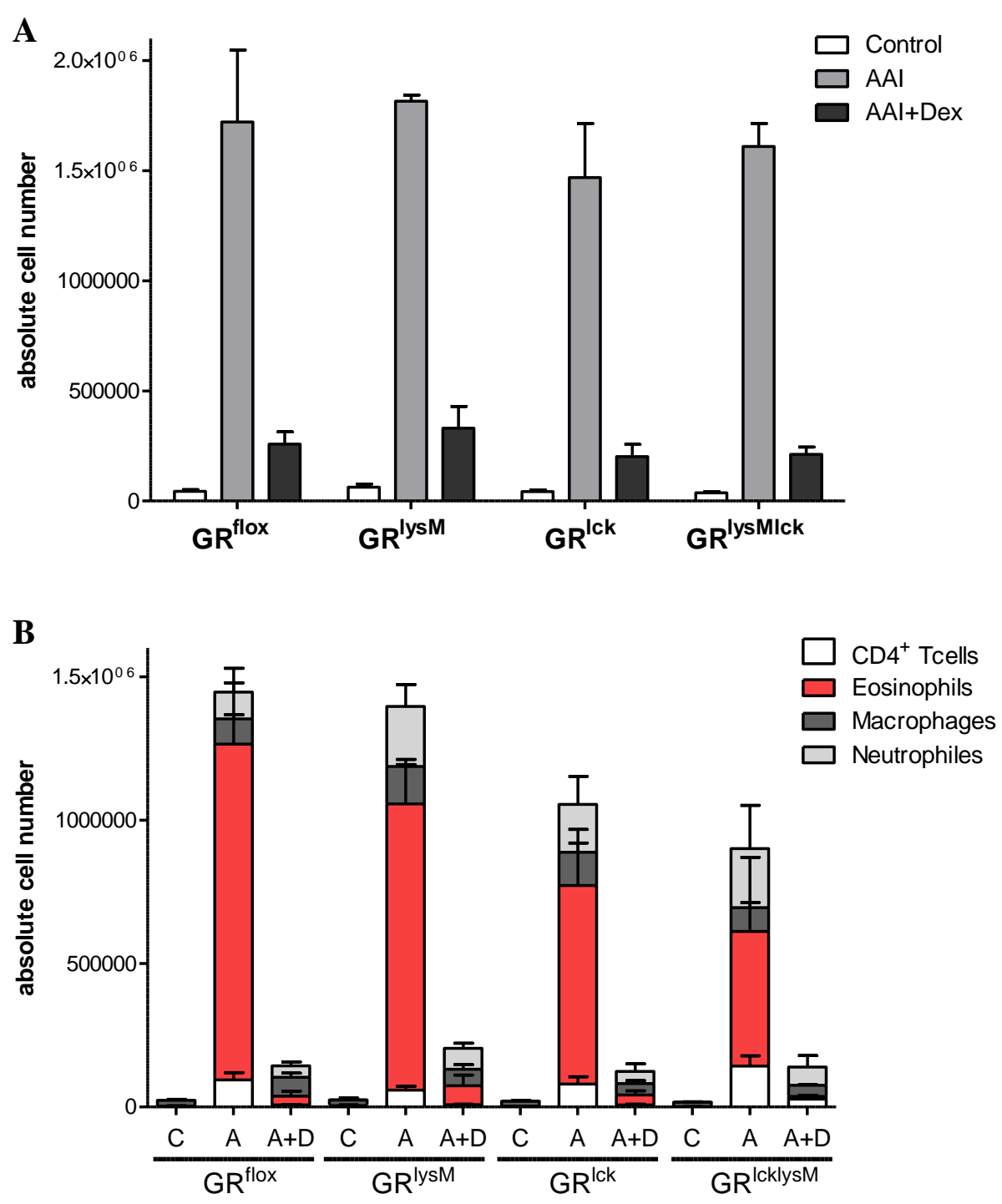

Figure 26: BAL cell numbers (A) and BAL cell composition (B) of $\mathbf{G R}^{\text {lysM }}, \mathbf{G R}^{\text {lck }}, \mathbf{G R}^{\text {lysMlck }}$ and GR $^{\text {flox }}$ mice on a C57BL/6 background. (A) The BAL of healthy (control, C), AAI (A) and Dextreated (AAI+Dex, A+D) mice was quantified for absolute cell numbers with the help of a Neubauer cell count chamber. (B) To investigate the BAL cell subpopulations the isolated BAL samples were analyzed by FACS. The relative percentages and absolute cell numbers of the BAL were used for the calculation of the absolute cell numbers of the subpopulations. The data represent the mean absolute cell number +/- SEM $\left(\right.$ GR $^{\text {flox }}$ control $n=9$; AAI $n=8$; AAI+Dex $n=9 ;$ GR $^{\text {lysM }}$ control $n=5$; AAI $n=6$; AAI+Dex $n=8 ; G^{\text {lck: }}$ control $n=6 ;$ AAI $n=6 ; A A I+D e x n=7 ; R^{\text {lysMlck }}$; control $n=2 ;$ AAI $n=2$; $\mathrm{AAI}+\operatorname{Dex} \mathrm{n}=3$ ) 
As it could be shown by FACS analysis of the BAL (Figure $26 \mathrm{~B}$ ), the cellular infiltration was dominated by eosinophils and could be strongly reduced by GC-therapy in all mutant mouse strains. The response to the therapy in mutant mice was similar and did not reveal any differences in cell composition or cell number reduction compared to wild type $\mathrm{GR}^{\text {flox }}$ mice.

\subsubsection{Proliferation test of of $G R^{\text {flox }}, G R^{\text {lysM }}, G^{\text {lck }}$ and $G R^{\text {lysMlck }}$ splenocytes ex vivo}

To analyze GC-effects on proliferation splenocytes were restimulated with Ova ex vivo.

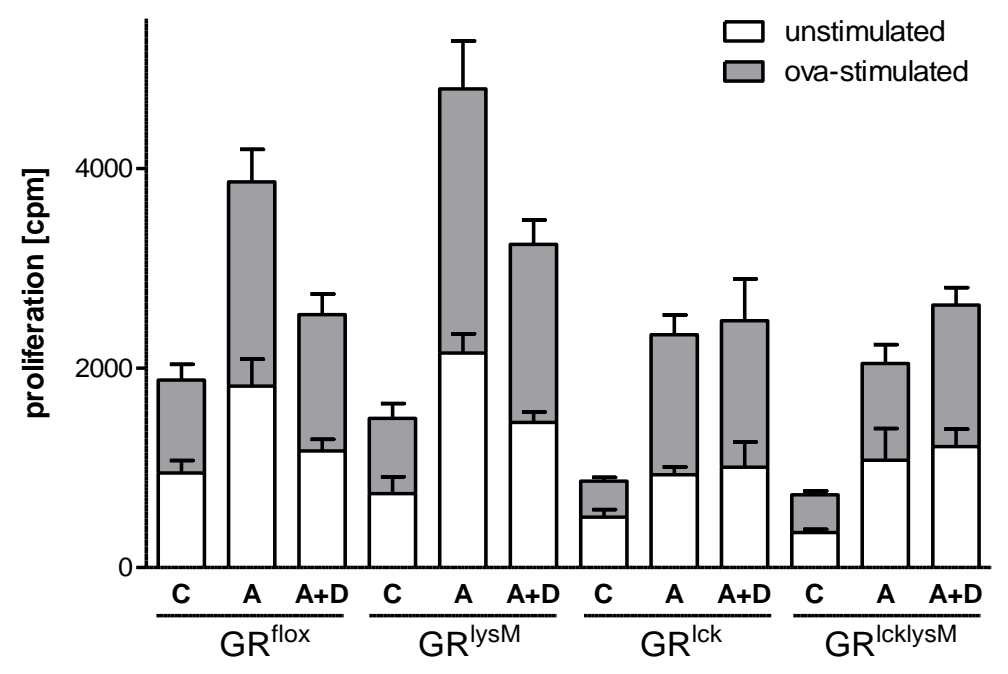

Figure 27: Proliferation rate of splenocytes from $\mathbf{G R}^{\text {lysM }}, \mathbf{G R}^{\text {lck }}, \mathbf{G R}^{\text {lysMlck }}$ and $\mathbf{G R}^{\text {flox }}$ mice after antigen-specific restimulation $\boldsymbol{e x}$ vivo. Proliferation activity of splenocytes from healthy control (C), AAI (A) and GC-treated (A+D) mice was determined after $72 \mathrm{hrs}$ incubation with antigen specific restimulation (Ova), compared to the basal proliferation (unstimulated cells). The cells were analysed for proliferation activity with ${ }^{3} \mathrm{H}$-thymidine incorporation assay. The results show the mean proliferation $(\mathrm{cpm})+/$ - SEM of triplicate measurements $\left(\mathrm{GR}^{\text {flox }}\right.$ control $\mathrm{n}=9$; AAI $\mathrm{n}=8$; AAI+Dex $n=9 ;$ GR $^{\text {lysM }}$ control $n=5$; AAI $n=6$; AAI+Dex $n=8 ;$ GR $^{\text {lck. }}$; control $n=6$; AAI $n=6$; AAI+Dex $n=7$; GR $^{\text {lysMlck }}$ : control $n=2 ;$ AAI $n=2 ; A A I+D e x n=3$ ).

Restimulation with Ova ex vivo led to an increased proliferation of splenocytes. Ova-specific proliferation could be reduced by GC-treatment only in samles of $\mathrm{GR}^{\text {flox }}$ and $\mathrm{GR}^{\text {lysM }}$ animals; GR ${ }^{\text {lck }}$ and GR ${ }^{\text {lcklysM }}$ splenocyte showed similar proliferation in GC-treated mice. IL-4 and IFNy production of splenocytes was too low for reliable analysis of cytokine release. 


\subsubsection{Immunoglobulin production of $G^{\mathrm{lysM}}, \mathrm{GR}^{\mathrm{lck}}$ and $G R^{\mathrm{lysMlck}}$}

The levels of Ova-specific IgE, IgG2a and IgG1 in serum samples of $\mathrm{GR}^{\text {flox }}$ and $\mathrm{GR}^{\text {lck }}$ or $\mathrm{GR}^{\text {lysMlck }}$ mice revealed successful induction of Ig production after immunization with Ova irrespective of which GR mutant strain was analyzed.
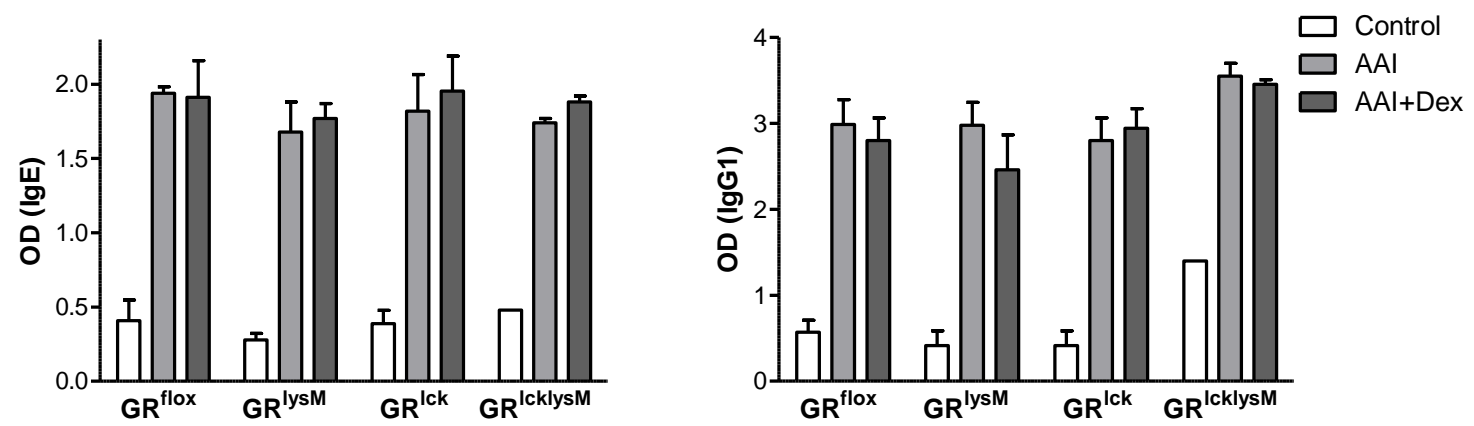

Figure 28: Immunoglobulin production and class switching in $\mathbf{G R}^{\text {lysM }}, \mathbf{G R}^{\text {lck }}, \mathbf{G R}^{\text {lcklysM }}$ and GR $^{\text {flox }}$ mice. The secretion of Ig isotypes was analyzed by Ova-specific Ig-ELISA. Serum samples for IgE measurement could be diluted 1:100 while the same serum samples for IgG1 detection were diluted 1:1000. The data were calculated as mean of OD $+/-\operatorname{SEM}\left(\mathrm{GR}^{\text {flox }}\right.$ control $n=9$; AAI $n=8$; AAI+Dex $n=9$; $\mathrm{GR}^{\text {lysM }}$ control $n=5$; AAI $n=6$; AAI+Dex $n=8$; $\mathrm{GR}^{\text {lck. }}$; control $n=6$; AAI $n=6$; AAI+Dex n=7; GR ${ }^{\text {lysMlck }}$; control n=2; AAI n=2; AAI+Dex n=3)

Immunoglobulin class switching occurred similarly after Ova-immunization in wild type $\mathrm{GR}^{\text {flox }}$ and all GR knockout mouse strains. GC-therapy had no effects on Ig production in any of the strains.

\subsubsection{Analysis of AAI Induction and Treatability of $G^{\mathrm{CD} 19}$ mice with GCs}

B cells are involved in asthmatic reactions and play an important role through the release of allergen-specific IgE which can activate mast cells, basophils and other inflammatory cells like macrophages and eosinophils (Sokol et al., 2009). The immunoglobulin class switching to the isotype IgE can be induced by the IL- 4 and IL-13 produced by $\mathrm{T}_{\mathrm{H}} 2$ cells.

To investigate the role of $\mathrm{B}$ cell suppression by GC-therapy in asthmatic reactions $\mathrm{GR}^{\mathrm{CD} 19}$ mice that lack GR expression on B cells were used.

\subsubsection{BAL analysis}

The analysis of the BAL from $\mathrm{GR}^{\mathrm{CD} 19}$ mutants demonstrated successfully induction of the inflammatory response by Ova-immunization. 


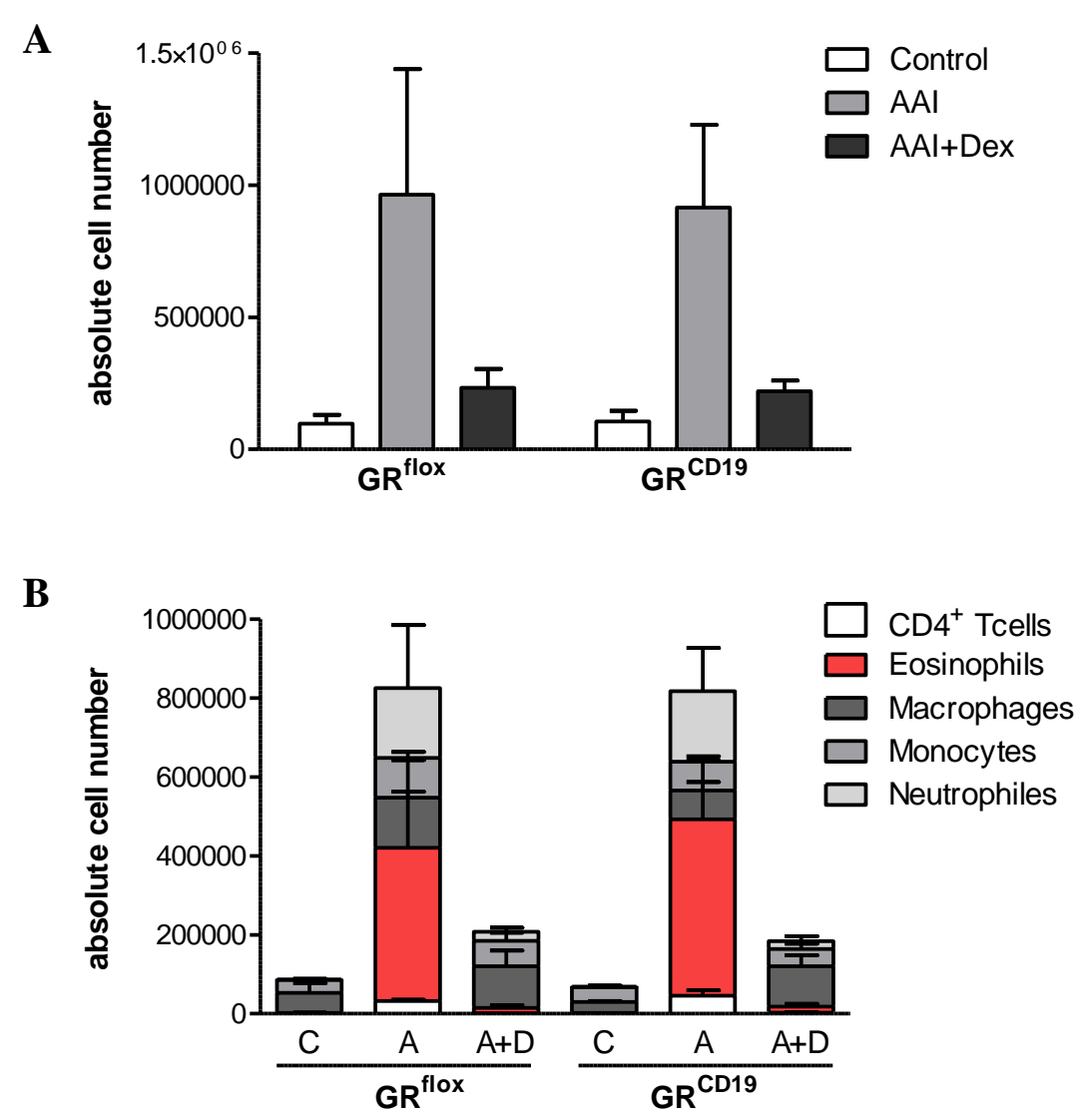

Figure 29: BAL cell numbers (A) and BAL cell composition (B) of $\mathrm{GR}^{\mathrm{CD19}}$ and $\mathrm{GR}^{\text {flox }}$ mice on a C57BL/6 background. The BAL of control (C), AAI (A) and Dex-treated (AAI+Dex) mice was quantified for absolute cell numbers with the help of a Neubauer cell count chamber. (B) To investigate BAL cell subpopulations the BAL samples were analyzed by FACS. The relative percentages and absolute cell numbers of the BAL were used for the calculation of the absolute cell numbers of the subpopulations. The data represent the mean absolute cell number +/- SEM deviation $\left(\mathrm{GR}^{\text {flox }}\right.$ : control $\mathrm{n}=2 ; \mathrm{AAI} n=2 ; \mathrm{AAI}+\mathrm{Dex} \mathrm{n}=4 ; \mathrm{GR}^{\mathrm{CD} 19}$ : control $\mathrm{n}=2 ; \mathrm{AAI} n=3 ; \mathrm{AAI}+\mathrm{Dex} \mathrm{n}=3$ )

Total cell numbers and the BAL cell composition were alike in $\mathrm{GR}^{\mathrm{flox}}$ and $\mathrm{GR}^{\mathrm{CD} 19}$ mice. Both mouse strains responded similarly to GC-therapy.

\subsubsection{Proliferation test of $G^{\mathrm{CD} 19}$ and $\mathrm{GR}^{\text {flox }}$ splenocytes ex vivo}

Proliferation activity was tested by ${ }^{3} \mathrm{H}$-thymidin incorporation assay. Proliferation was higher in Ova-immunized mice and reduced by GC-treatment. $\mathrm{GR}^{\mathrm{flox}}$ and $\mathrm{GR}^{\mathrm{CD} 19}$ mice behaved qualitatively similar. 


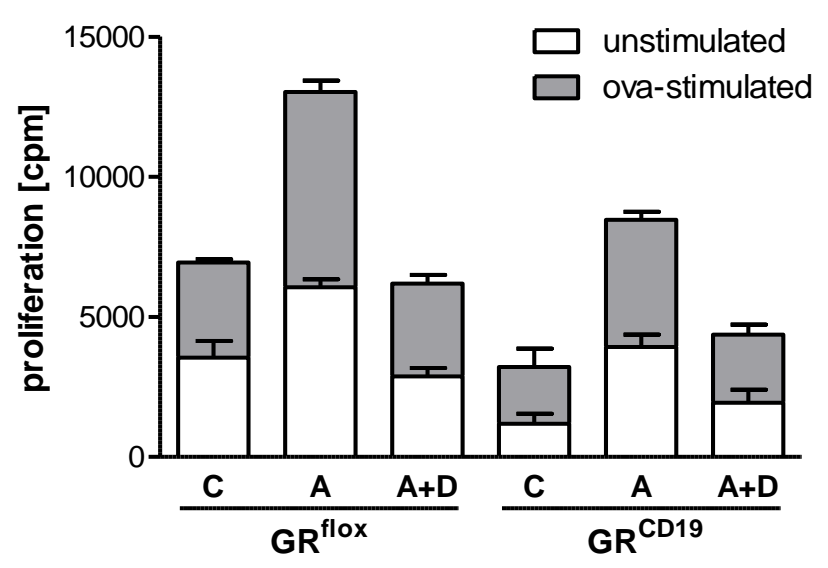

Figure 30: Proliferation of splenocytes from $G^{\mathrm{CD19}}$ and $\mathbf{G R}^{\text {flox }}$ mice after antigen-specific restimulation ex vivo. Proliferation activity of splenocytes from healthy control (C), AAI (A) and GC-treated $(\mathrm{A}+\mathrm{D})$ mice was determined after $72 \mathrm{hrs}$ incubation with antigen specific restimulation (Ova), compared to the basal proliferation (unstimulated cells). The cells were analysed for proliferation activity by ${ }^{3} \mathrm{H}$-thymidine incorporation assay. Results show the mean proliferation activity $(\mathrm{cpm})+$ - SEM of triplicate measurements. $\left(\mathrm{GR}^{\text {flox }}\right.$ : control $\mathrm{n}=2$; AAI $n=2$; AAI+Dex $n=4$; $\mathrm{GR}^{\mathrm{CD} 19}$ : control $\mathrm{n}=2 ; \mathrm{AAI} n=3 ; \mathrm{AAI}+\operatorname{Dex} \mathrm{n}=3$ )

\subsubsection{Immunoglobulin production in $G_{R}{ }^{\mathrm{CD} 19}$ mice}

In order to test immunoglobulin class switching after Ova-immunization an Ova-specific IgELISA was made with serum samples from $\mathrm{GR}^{\text {flox }}$ and $\mathrm{GR}^{\mathrm{CD} 19}$ mice.

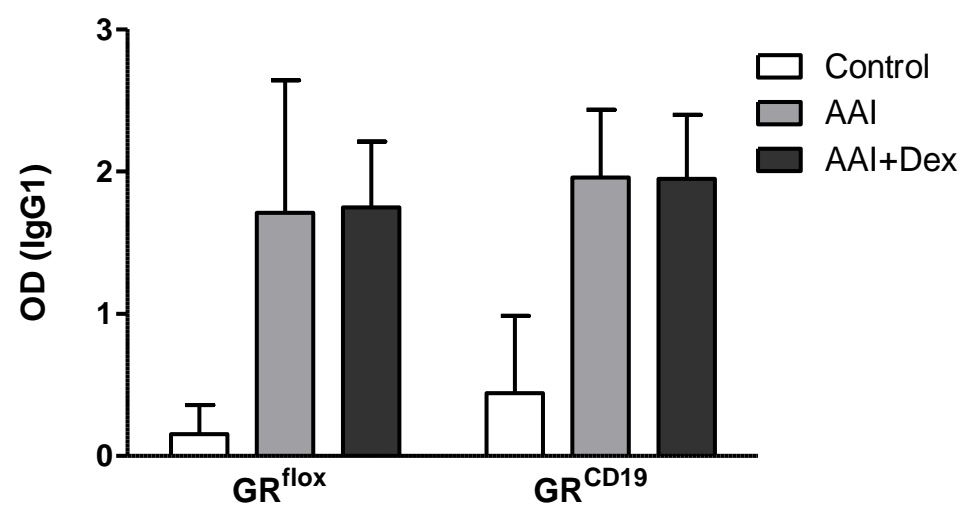

Figure 31: Immunoglobulin production and class switching in $\mathbf{G R}^{\text {flox }}$ and $\mathbf{G R}^{\mathbf{C D 1 9}}$ mice. The Ig secretion and isotype switch were analyzed by Ova-specific Ig-ELISA with isolated serum from healthy (control), AAI and Dexamethasone-treated (AAI+Dex) mice. The serum samples for IgG1 detection was diluted 1:1000. The data were calculated as the mean of the OD +/- SEM $\left(\mathrm{GR}^{\text {flox }}\right.$ : control $n=2 ;$ AAI $n=2 ;$ AAI+Dex $n=4 ; \mathrm{GR}^{\mathrm{CD} 19}$; control $n=2 ;$ AAI $n=3 ; A A I+D e x n=3$ )

This analysis revealed that Ova-specific IgG1 was made in similar amounts in $\mathrm{GR}^{\text {flox }}$ and $\mathrm{GR}^{\mathrm{CD} 19} \mathrm{AAI}$ and Dexamethasone-treated mice. 


\subsubsection{Analysis of AAI induction and treatability of $G^{\mathrm{CD11c}}$ and $\mathrm{GR}^{\text {flox }}$ with GCs}

DCs play an important role in generating $\mathrm{T}$ cell responses (Belz et al., 2004) and initiating and perpetuating $\mathrm{T}$ cell hyperresponsiveness associated with asthmatic reactions (van Rijt et al., 2005; Hammad et al., 2010). DCs as professional antigen presenting cells in the lungs transport the majority of antigens to the draining LNs and can direct $\mathrm{T}$ cell differentiation towards a $\mathrm{T}_{\mathrm{H}} 2$ response during an asthmatic reaction in the lungs. To understand the importance of DC suppression by GC-therapy during treatment of the asthma-like reaction $\mathrm{GR}^{\mathrm{CD} 11 \mathrm{c}}$ mice were used where GR-expression is almost completely ablated in DCs and partially in macrophages and T cells.

\subsubsection{BAL analysis}

The induction of $\mathrm{AAI}$ in $\mathrm{GR}^{\text {flox }}$ and $\mathrm{GR}^{\mathrm{CD} 11 \mathrm{c}}$ mice led to a strong inflammatory response characterized by high numbers of infiltrating cells and strongly increased eosinophil number in the lungs.
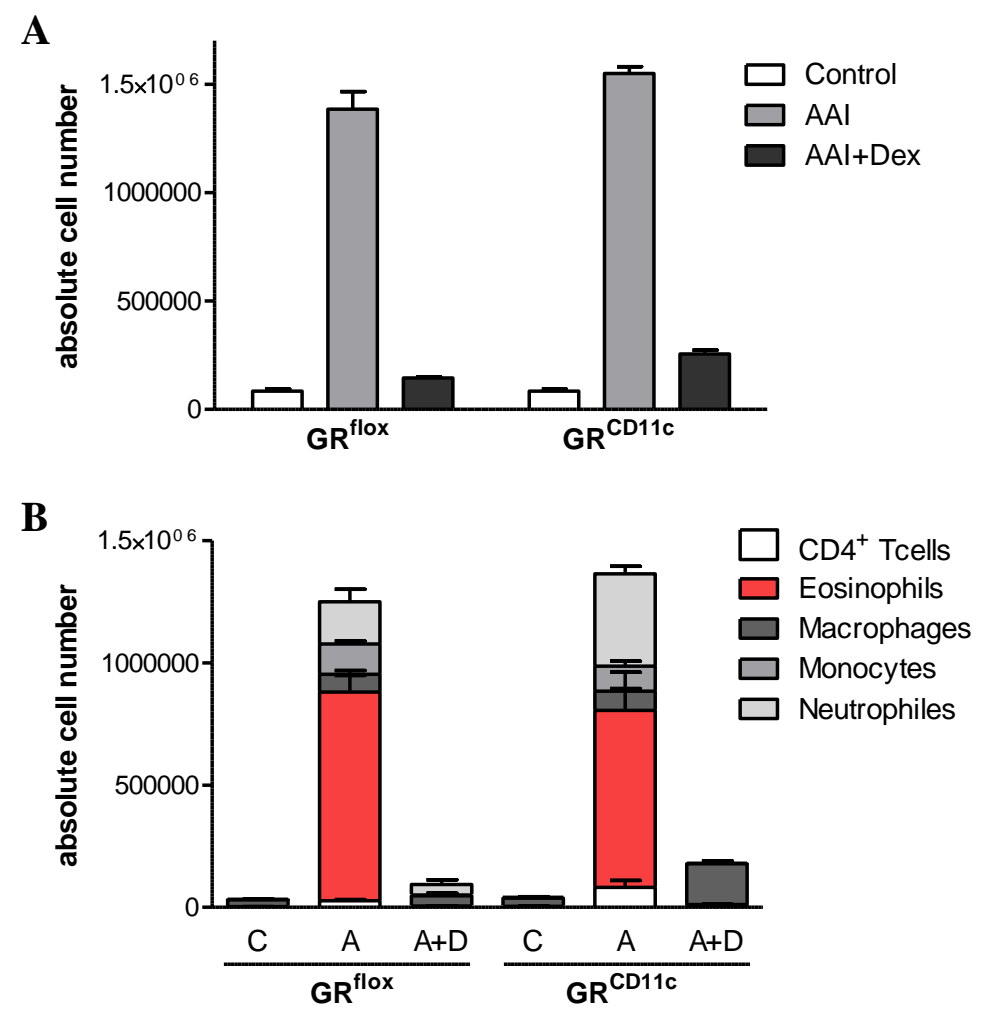

Figure 32: BAL cell numbers (A) and BAL cell composition $(B)$ in $\mathbf{G R}^{\mathrm{CD11c}}$ and $\mathbf{G R}^{\text {flox }}$ mice on a C57BL/6 background. (A) The BAL of healthy (C), AAI and Dexamethasone-treated (AAI+Dex) mice was quantified for the absolute cell number with help of a Neubauer cell count chamber.

(B) To investigate the BAL cell subpopulations the isolated BAL samples were analyzed by FACS. The relative percentages and absolute cell numbers of the BAL were used for the calculation of the absolute cell numbers of the subpopulation. The data represent the mean absolute cell number $+/-$ SEM $\left(\mathrm{GR}^{\text {flox }}\right.$ : C n=2; AAI n=4; AAI+Dex n=5; GR ${ }^{\mathrm{CD} 11 \mathrm{c}}$ : C n=2, AAI n=2; AAI+Dex n=2) 
Leukocyte infiltration in the lungs of $\mathrm{GR}^{\mathrm{CD} 11 \mathrm{c}}$ mice was comparable to GRflox control mice. The only dissimilarity was the evaluated number of neutrophils in the BAL. While the infiltration in asthmatic wild type animals was clearly dominated by eosinophils, the BAL of asthmatic $\mathrm{GR}^{\mathrm{CD} 11 \mathrm{c}}$ mice contained more than $25 \%$ of neutrophils. However, the GC-effect on lung infiltration was similar in $\mathrm{GR}^{\text {flox }}$ and $\mathrm{GR}^{\mathrm{CD} 11 \mathrm{c}}$ animals.

\subsubsection{Proliferation and cytokine release by $G^{\text {CD11c }}$ splenocytes}

To examine the influence of GC on the proliferation activity of splenocytes and the dependence of this reaction on DCs, the data obtained from restimulated $\mathrm{GR}^{\text {flox }}$ splenocytes from $\mathrm{GC}$-treated asthmatic mice were compared to results from $\mathrm{GR}^{\mathrm{CD} 11 \mathrm{c}}$ samples.

Restimulation of wild type and $\mathrm{GR}^{\mathrm{CD} 11 \mathrm{c}}$ splenocytes from asthmatic mice led to an increased proliferation and elevated production of IL-2. The splenocytes of both tested genotypes could be successfully suppressed in their proliferation activity and IL-2 production by GCtherapy.
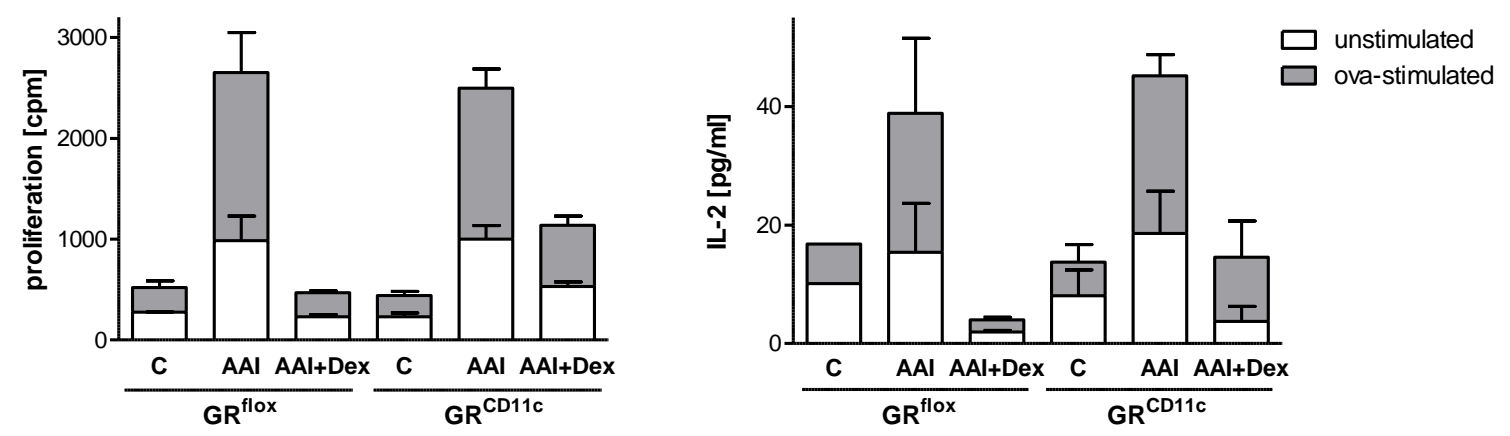

Figure 33: Proliferation and IL-2 secretion of splenocytes in GR $^{\text {CD11c }}$ and GR $^{\text {flox }}$ mice after antigen-specific restimulation $\boldsymbol{e x}$ vivo. The proliferation activity of splenocytes from healthy control, AAI and GC-treated (AAI+Dex) mice was determined after $72 \mathrm{hrs}$ incubation with antigen specific restimulation (Ova), compared to the basal proliferation (unstimulated cells). The cells were analysed for proliferation activity with ${ }^{3} \mathrm{H}$-thymidine incorporation assay. IL-2 production was measured by ELISA using cell culture supernatants. The results show the mean proliferation activity (cpm) and the IL-2 concentration (pg/ml) +/- SEM of triplicate measurements. $\left(\mathrm{GR}^{\text {flox }}: \mathrm{C} \mathrm{n}=2\right.$; AAI $\mathrm{n}=4 ; \mathrm{AAI}+\operatorname{Dex} \mathrm{n}=5 ; \mathrm{GR}^{\mathrm{CD} 11 \mathrm{c}}$ : $\mathrm{C}$ n=2, AAI n=2; AAI+Dex n=2)

The ${ }^{3} \mathrm{H}$-thymidine incorporation assay and the IL-2 secretion analysis showed that the reduction of proliferation and IL-2 secretion by splenocytes was not dependent on the GR in DCs. 


\subsubsection{Immunoglobulin production and class switching in $G R^{\mathrm{CD} 11 \mathrm{c}}$ and $\mathbf{G R}^{\mathrm{wt}}$ mice}

In order to analyze the Ova-specifici immunoglobulins in $\mathrm{GR}^{\mathrm{CD} 11 \mathrm{c}}$ mice serum samples were used for $\operatorname{IgE}, \operatorname{Ig} \mathrm{G} 2 \mathrm{a}$ and $\operatorname{IgG} 1$ detection.
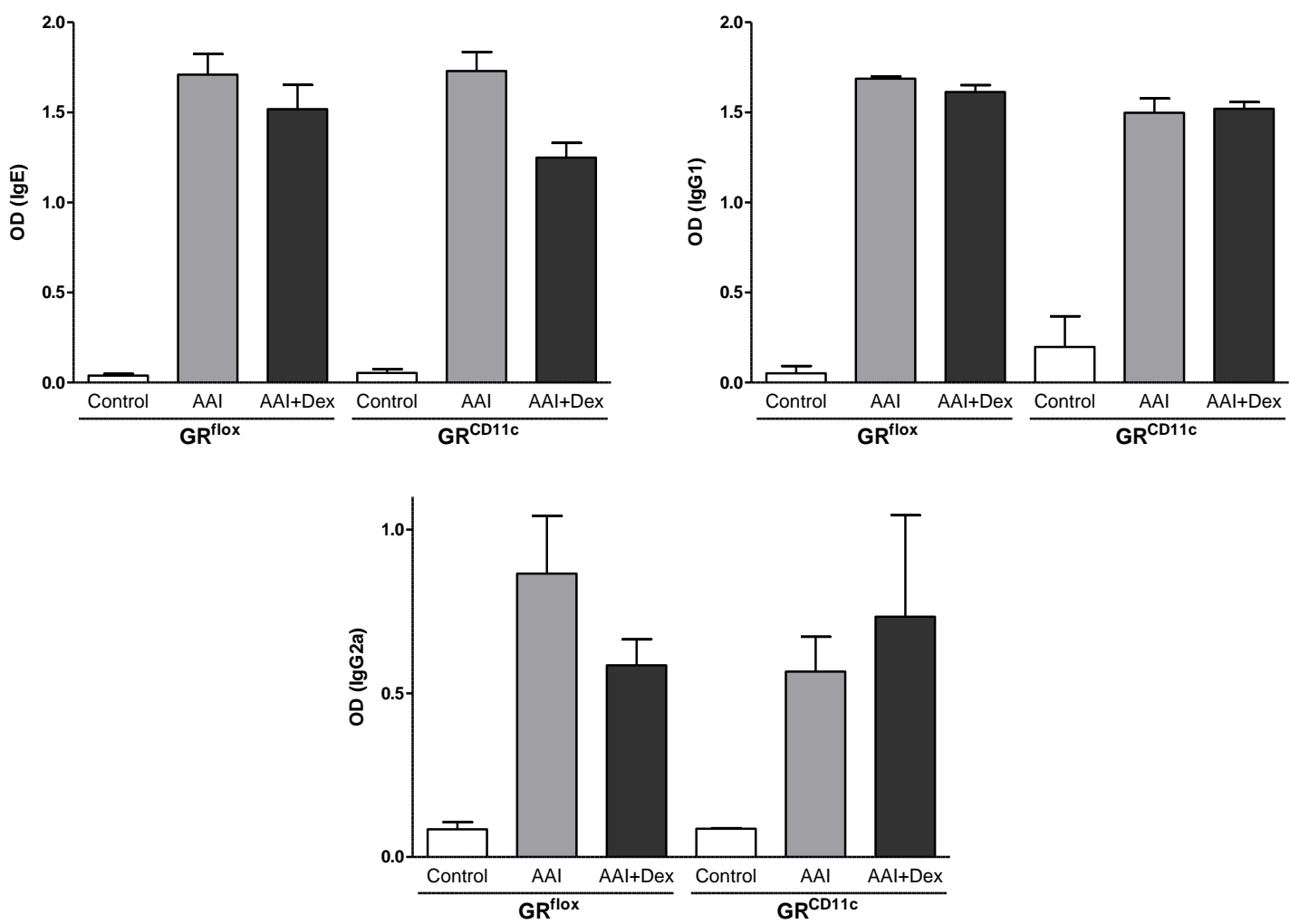

Figure 34: Immunoglobulin isotype levels in the serum of $\mathbf{G R}^{\mathrm{CD11}}$ and $\mathbf{G R}^{\text {flox }}$ mice on a C57BL/6 background. The Ig secretion and isotype switch were analyzed by Ova-specific IgELISA with the serum from healthy (control), AAI and Dexamethasone-treated (AAI+Dex) mice. The dilution of the serum was dependent on the Ig isotype: $\operatorname{IgE} 1: 50, \operatorname{IgG} 2$ a 1:1000, $\operatorname{IgG} 1$ 1:100000. The data show the mean OD +/- SEM (GR flox: Control $n=2 ;$ AAI $n=4 ; A A I+D e x n=5 ; \mathrm{GR}^{\mathrm{CD} 11 \mathrm{c}}$ : C $\mathrm{n}=2$, AAI $\mathrm{n}=2 ; \mathrm{AAI}+\mathrm{Dex} \mathrm{n}=2$ )

Ova-specific immunoglobulins of all these isotypes were strongly increased after Ovaimmunization. Like in other experiments GC-therapy had no effects on Ig production in $\mathrm{GR}^{\mathrm{CD} 11 \mathrm{c}}$ and $\mathrm{GR}^{\text {flox }}$ control mice. 


\subsubsection{Analysis of the AAI induction and treatability of $\mathrm{Kit}^{\mathrm{W}-\mathrm{sh} / \mathrm{W}-\mathrm{sh}}$ mice with GCs}

Mast cells are one of the most important player in inflammatory reactions during asthma. These cells make up only a very small part of the BAL cells but within the airway tissue they encompass more than $20 \%$ of all inflammatory cells. Mast cells are located on the surface and within the airways and can directly respond to provocative stimuli.

To investigate the importance of mast cells suppression by GCs during the therapy of allergic response in the lungs the mast cell deficient mutant mice $K i t^{W-s h / W-s h}$ mice (in following text designated as cKit) were analysed.

\subsubsection{BAL analysis}

cKit mice developed a strong inflammation after immunisation and challenge with Ova. The inflammatory response in the employed asthma-model was not impaired by the absence of mast cells in cKit mice.
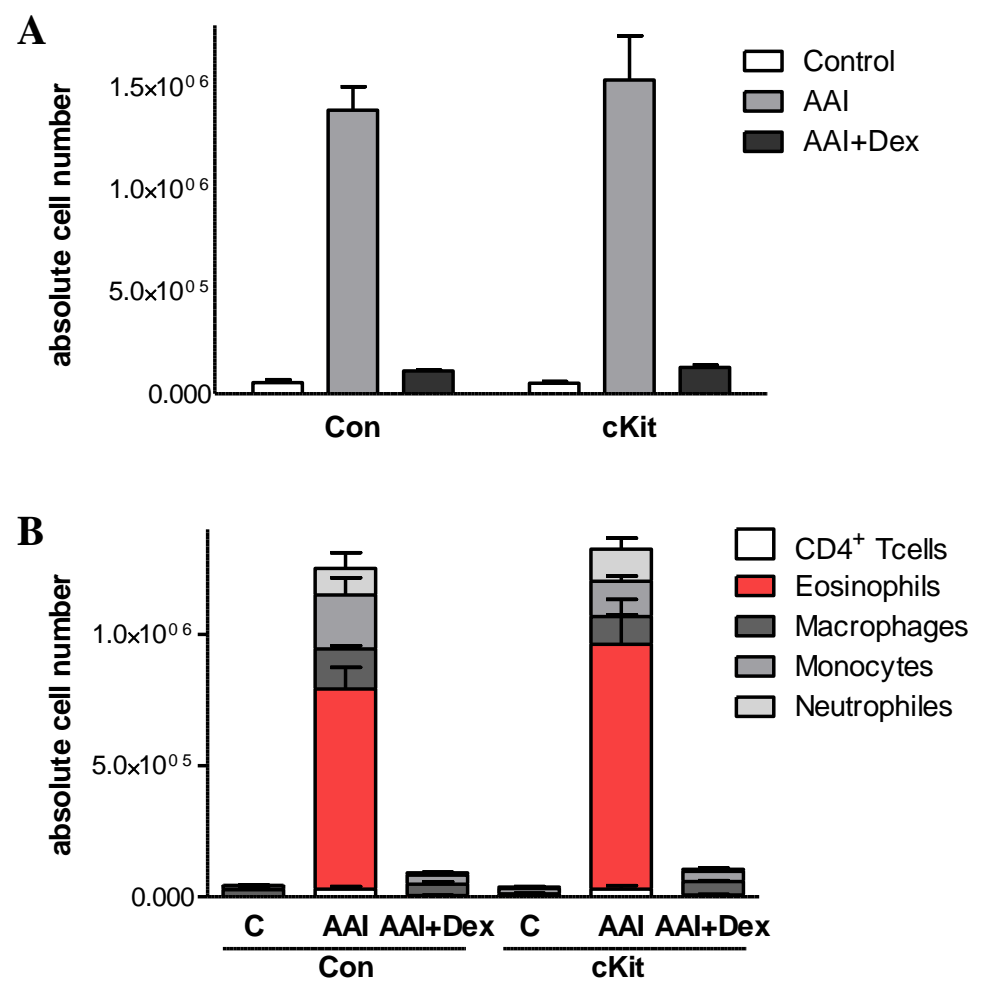

Figure 35: BAL cell numbers (A) and BAL cell composition (B) of cKit and wild type control mice (con) on a C57BL/6 background. (A) The BAL cells of healthy (Control), AAI and Dextreated (AAI+Dex) mice were quantified for the absolute cell numbers with the help of a Neubauer cell count chamber. (B) To investigate the BAL cell subpopulations the isolated BAL samples were analyzed by FACS. The relative percentages and the absolute cell numbers of the BAL were used for the calculation of the absolute cell number of the subpopulation. The data represent the mean absolute cell number +/- SEM (Con: control $n=3$; AAI $n=4$; AAI+Dex $n=6$; cKit: control $n=3$; AAI $\mathrm{n}=5 ; \mathrm{AAI}+\mathrm{Dex} \mathrm{n}=6$ ) 
Infiltration based on absolute cell numbers and the composition of the BAL cells was similar in wild type controls and cKit mice. Both mouse strains response to the asthma-induction with a cellular influx into the lungs dominated by eosinophils. The absolute number of BAL cells was also comparable between both genotypes.

GC-therapy successfully suppressed cellular infiltration and reduced the absolute cell number of the infiltrate from more than $1.5 \times 10^{6}$ to less than 200000 cells and eosinophilia (from more than 800000 eosinophils in the asthmatic BAL to 10000 cells in GC-treated animals).

\subsubsection{Proliferation and IL-2 secretion by splenocytes from cKit mice}

To analyze the effects of GCs on proliferation and priming, splenocytes from healthy, asthmatic and GC-treated mice were isolated and used for a ${ }^{3} \mathrm{H}$-thymidine incorporation assay and the analysis of IL-2 by ELISA.
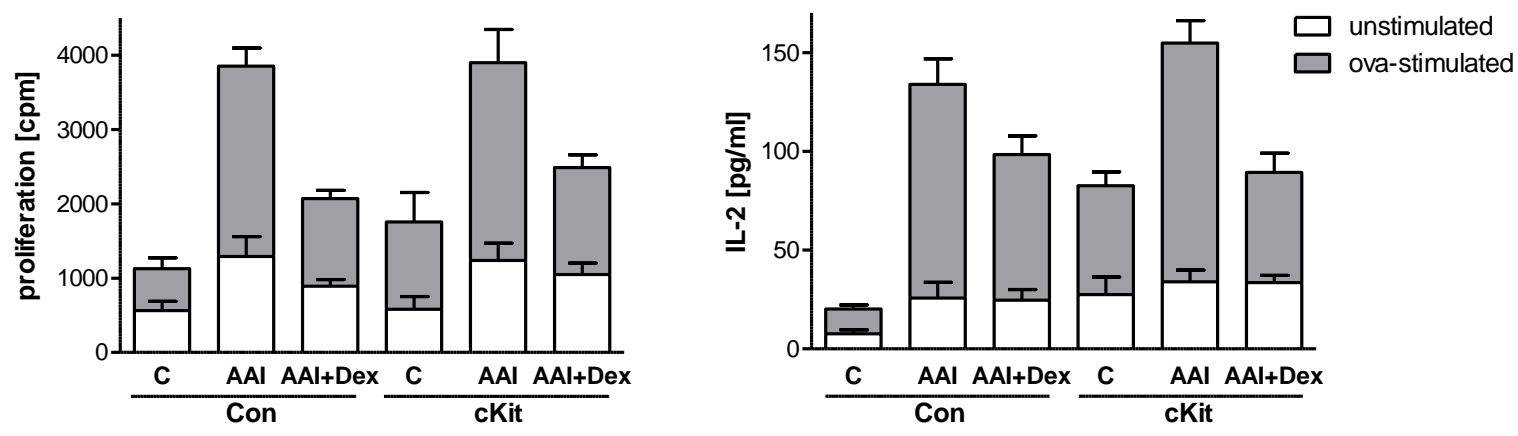

Figure 36: Proliferation and priming in wild type controls (con) and cKit mice after antigenspecific restimulation ex vivo. Proliferation of splenocytes from healthy (C), AAI and GC-treated (AAI+Dex) mice was determined after $72 \mathrm{hrs}$ incubation with antigen-specific restimulation (Ova), compared to the basal proliferation (unstimulated cells). The cells were analysed for proliferation activity with ${ }^{3} \mathrm{H}$-thymidine incorporation assay. The IL-2 release was determined by ELISA. The results show the mean proliferation (cpm) or IL-2 concentration +/- SEM of triplicate measurements. (Con: control n=3; AAI n=4; AAI+Dex n=6; cKit: control n=3; AAI n=5; AAI+Dex n=6)

The ${ }^{3} \mathrm{H}$-thymidine assay and the results from the IL-2 ELISA showed enhanced proliferation and increased IL-2 secretion in samples of AAI mice. This could be prevented by GCtherapy of wild type and cKit mice. 


\subsection{The role of DNA-binding-dependent transactivation function of the GR for GC-therapy of AAI}

It is well documented that most GC effects are mediated by the GC receptor (GR) via transactivation or transrepression. To determine the relevance of these two GR functions for GC intervention in asthma, $\mathrm{GR}^{\mathrm{dim}}$ mice (on a BALB/c background) were tested for the treatment efficiency of Dexamethasone. Because of a point mutation $\mathrm{GR}^{\mathrm{dim}}$ mice express a modified form of the GR that is no longer able to dimerise and therefore lacks DNA-binding dependent gene regulation.

\subsubsection{BAL analysis of $\mathbf{G R}^{\mathrm{dim}}$ mice}

In order to investigate the molecular mechanism of GC-therapy of AAI, GR ${ }^{\mathrm{wt}}$ and $\mathrm{GR}^{\mathrm{dim}}$ mice were used for induction and treatment of a mouse model of Asthma.

First of all, the numbers of the BAL cells was determined.

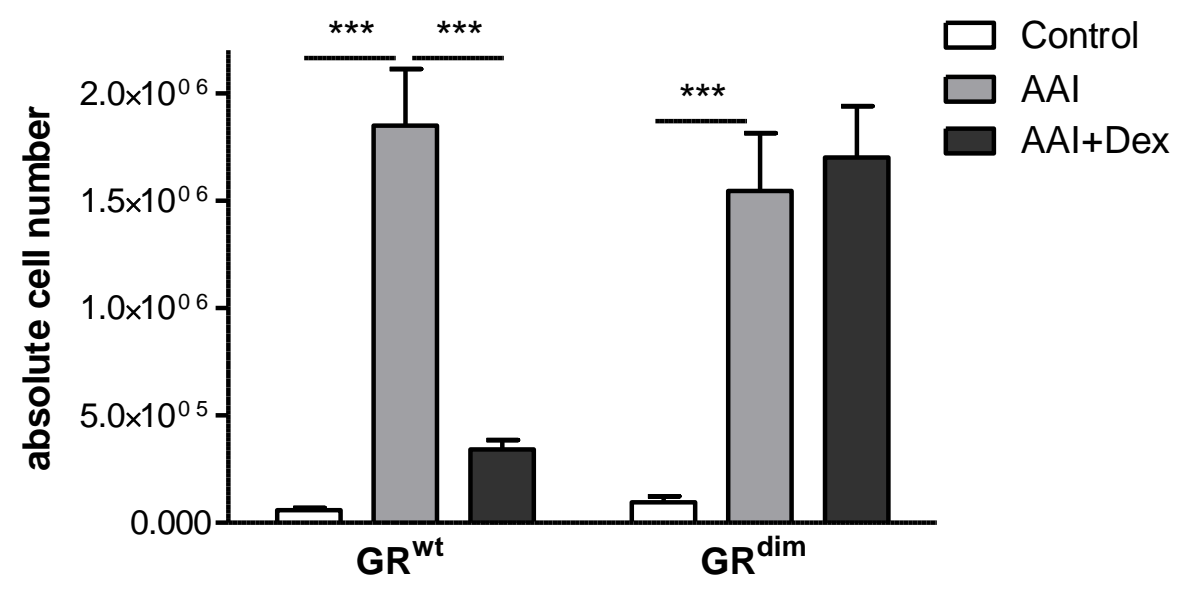

Figure 37: Absolute cell numbers in the BAL of healthy (control), AAI and GC-treated (AAI+Dex) $\mathbf{G R}^{\mathrm{wt}}$ and $\mathbf{G R}^{\mathrm{dim}}$ mice. The AAI was induced by repeated Ova-immunisation and challenges, the AAI+Dex animals were treated with Dexamethasone. The BAL was isolated $40 \mathrm{hrs}$ after the last Ova-challenge. The absolute cell number in the BAL samples was determined using a Neubauer cell count chamber. The data represent the mean of cell number +/- SEM (GR ${ }^{\mathrm{wt}}$ : Control $n=13 ;$ AAI n=13; AAI+Dex n=14; GR $^{\text {dim }}$ : Control n=13; AAI n=12; AAI+Dex n=17).

After the induction of the allergic response the BAL cell number in $\mathrm{GR}^{\mathrm{wt}}$ and $\mathrm{GR}^{\mathrm{dim}}$ mice was strongly increased and the BAL in Ova-sensitised mice contained more than $1.5 \times 10^{6}$ cells while the control healthy mice had only around 100000 cells in the BAL. Treatment of the inflammatory reaction with Dexamethasone reduced the absolute cell number in the BAL of $\mathrm{GR}^{\mathrm{wt}}$ mice while GC-therapy of $\mathrm{GR}^{\mathrm{dim}}$ mice had no influence on the number of lung-infiltrating cells (Figure 37). 
The observation that the number of infiltrating cells in the lungs was increased could be corroborated by FACS analysis of the cell composition. While healthy mice in both cases have a large population of macrophages in the lungs and nearly no eosinophils, the asthmatic mice developed a strong eosinophilia with about $70 \%$ of eosinophils in the BAL. Because of this strong eosinophilia the relative percentage of other cell type were strongly reduced in comparison to the BAL from healthy mice. This replacement in cell population arrangement was seen in the both genotypes.

A

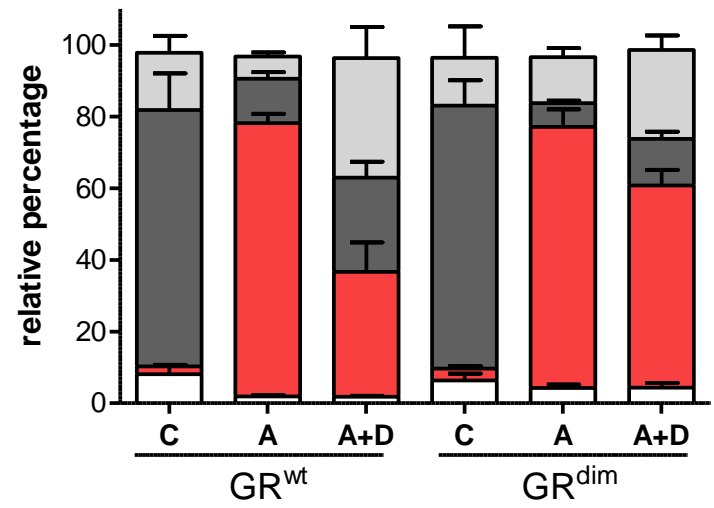

B

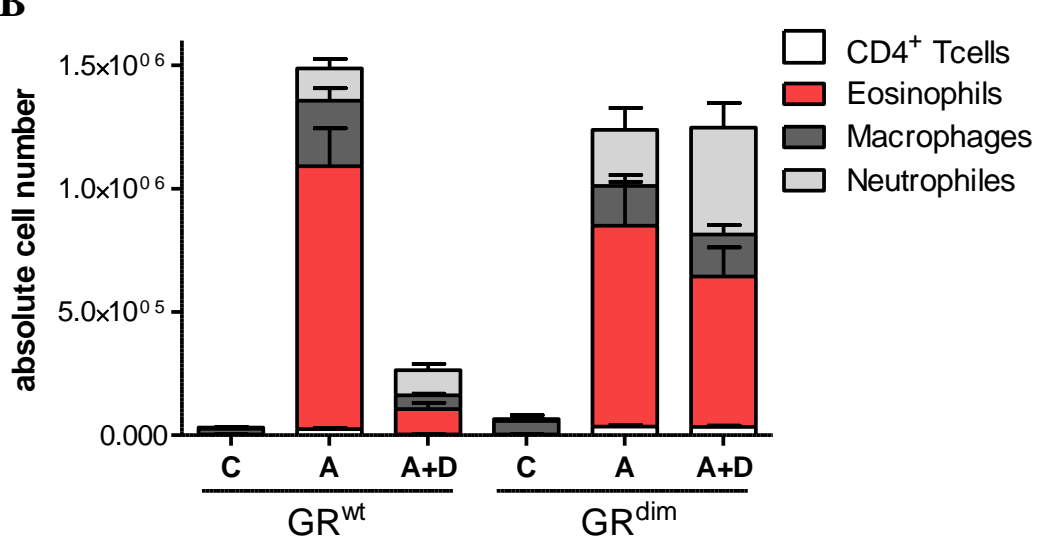

Figure 38: Analysis of the absolute cell number and the relative percentage of different subpopulations in BAL samples of $\mathbf{G R}^{\mathrm{wt}}$ and $\mathbf{G R}^{\mathrm{dim}}$ mice. The relative percentage of $\mathrm{T}$ helper cells, eosinophils, macrophages and monocytes were determined by FACS analysis. Based on the relative percentage of the subpopulations and the absolute cell numbers of the BAL cells, the absolute cell number of each subpopulation could be calculated. The diagrams represent the mean of absolute cell numbers +/- SEM (GR ${ }^{\text {wt }}$ : Control $n=9$; AAI $n=11$; AAI+Dex $n=11$; GR $^{\text {dim }}$ : Control $\mathrm{n}=13$; AAI $\mathrm{n}=10$; AAI+Dex $n=14)$.

The GC-treatment could suppress the infiltration of the lungs with eosinophils only in $\mathrm{GR}^{\mathrm{wt}}$ mice. While the cell number of eosinophils in AAI was significantly reduced from more than $1 \times 10^{6}$ cells in $\mathrm{GR}^{\mathrm{wt}}$ mice to about 100000 eosinophils in Dex-treated mice, the treatment of $\mathrm{GR}^{\mathrm{dim}}$ led to minimal changes. After GC-therapy the BAL of $\mathrm{GR}^{\mathrm{dim}}$ mice still consist of more than $60 \%$ of eosinophils and the changes in eosinophil numbers after GCtherapy were not significant. The numbers of neutrophils was not reduced after 
Dexamethasone-treatment in both genotypes. The relative percentage of this population was increased after therapy with GCs.

\subsubsection{Proliferation and cytokine release by $\mathbf{G R}^{\mathrm{dim}}$ splenocytes ex vivo}

To examine the effects of GC-treatment on the proliferation and priming of splenocytes antigen-specific restimulation was used ex vivo. This restimulation of splenocytes with Ovalbumin led to an increased proliferation of Ova-specific cells of both genotypes. This was demonstrated by ${ }^{3} \mathrm{H}$-thymidine incorporation assay and the analysis of IL-2 production by ELISA. The ${ }^{3} \mathrm{H}$-thymidine incorporation assay showed no differences in the proliferation between AAI and Dexamethasone-treated animals. However, the analysis of IL-2 in the supernatant of cells from AAI in $\mathrm{GR}^{\mathrm{wt}}$ mice showed not only strongly increased secretion of this cytokine but also a significantly reduction of its production in Dexamethasone-treated animals. This effect of Dexamethasone was not observed for $\mathrm{GR}^{\mathrm{dim}}$ splenocytes.
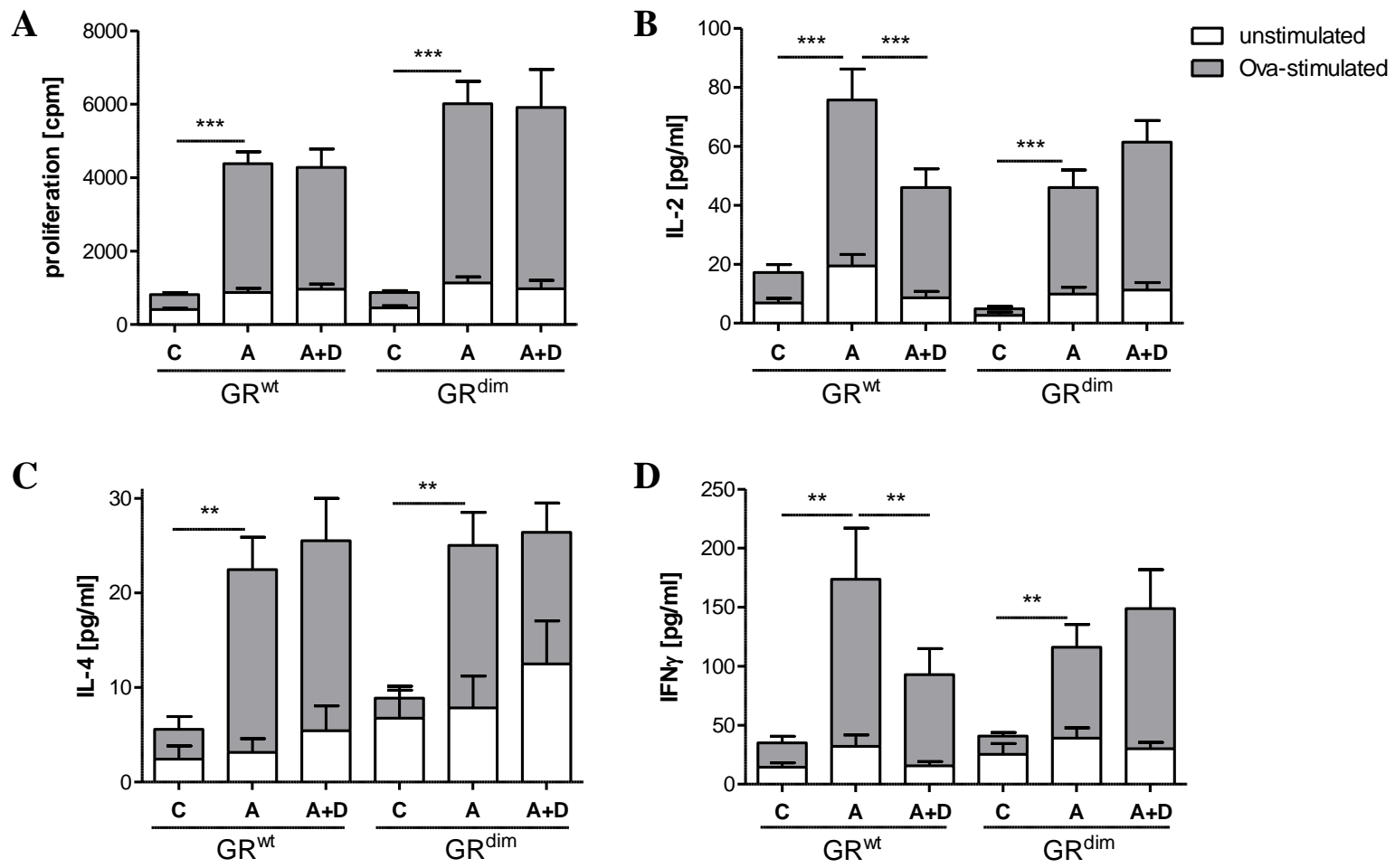

Figure 39: Proliferation and cytokine release by splenocytes of healthy, AAI and GC-treated $\mathbf{G R}^{\mathrm{wt}}$ and GR ${ }^{\mathrm{dim}}$ mice ex vivo. Proliferation and cytokine release was determined for splenocytes (from healthy=C, AAI=A and GC-treated A+D mice) after $72 \mathrm{hrs}$ with antigen specific restimulation with Ova, compared to the basal proliferation and cytokine secretion (unstimulated cells). The cells were analysed for proliferation activity using a ${ }^{3} \mathrm{H}$-thymidine incorporation assay (data shown as cpm-mean +/-SEM). The cell culture supernatants were analyzed for IL-2, IL-4 and IFN $\gamma$ by ELISA. Results shown are the mean concentration $+/$ - SEM of triplicate measurements $\left(\mathrm{GR}^{\mathrm{wt}}\right.$ : Control n=9; AAI n=11; AAI+Dex n=11; GR $^{\text {dim }}$ : Control $n=13$; AAI $n=10$; AAI+Dex $\left.n=14\right)$. 
The Ova-stimulated splenocytes from asthmatic $\mathrm{GR}^{\mathrm{wt}}$ and $\mathrm{GR}^{\mathrm{dim}}$ mice showed an increased secretion of IL-4 and IFN $\gamma$. While the splenocytes from GC-treated animals of both genotypes showed no significantly increase in IL-4 secretion, the IFN $\gamma$ production showed a different regulation. The splenocytes from asthmatic $\mathrm{GR}^{\mathrm{wt}}$ mice secreted less IFN $\gamma$ after Dexamethasone-therapy while the $\mathrm{GR}^{\mathrm{dim}}$ splenocytes from GC-treated mice produced more IFN $\gamma$ compared to the production of splenocytes from AAI mice.

\subsubsection{Antigen-specific immunoglobulin production}

To analyse the Ig production after induction of an asthma-like reaction and GC-treatment in $\mathrm{GR}^{\mathrm{wt}}$ and GR ${ }^{\mathrm{dim}}$ mice Ova-specific Ig ELISAs were used.
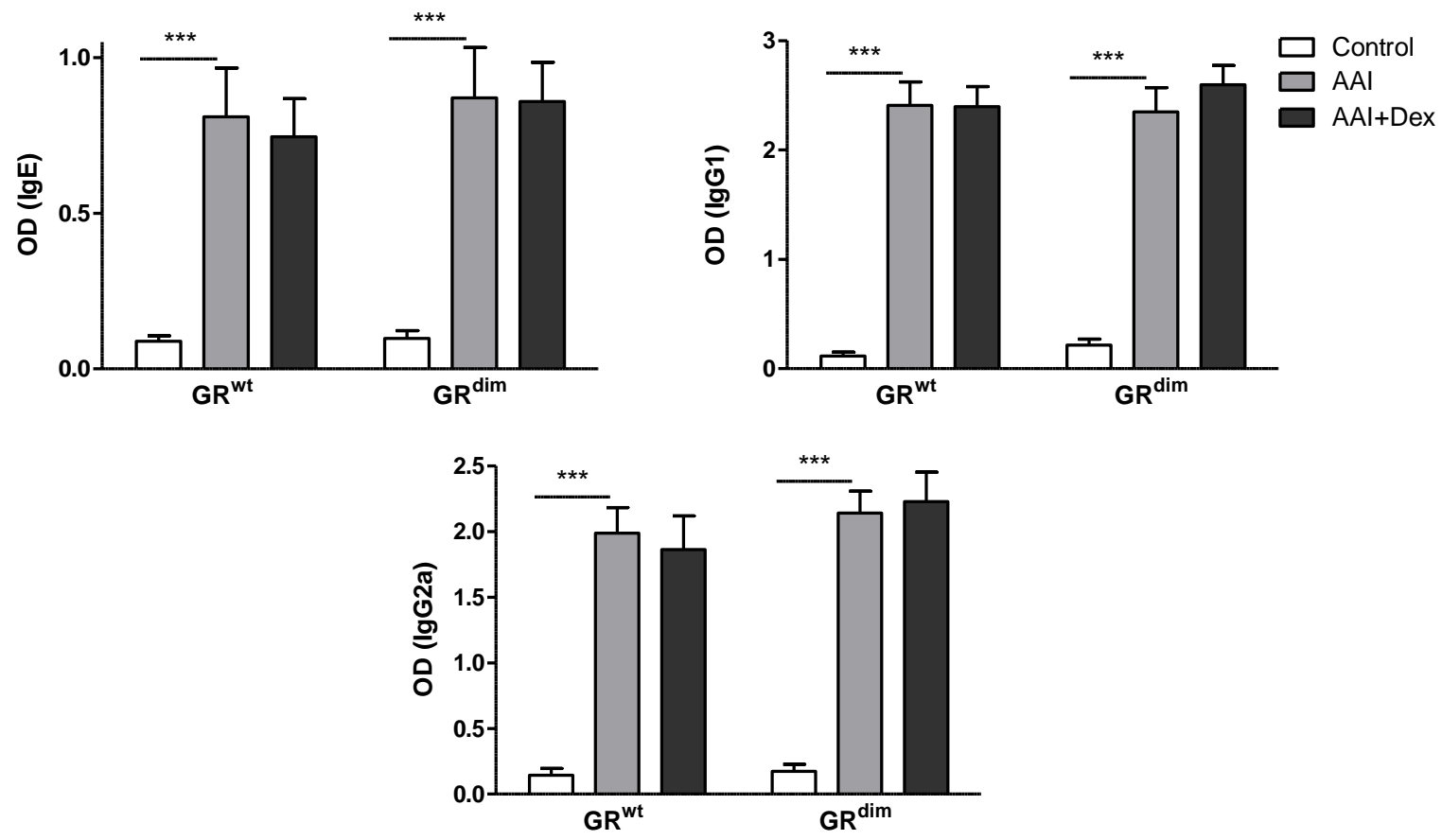

Figure 40: Ova-specific immunoglobulin production in healthy, AAI and GC-treated AAI GR ${ }^{\mathrm{wt}}$ and $\mathbf{G R}^{\mathrm{dim}}$ mice. For detection and measurement of Ig serum was collected from each animal $40 \mathrm{hrs}$ after the last challenge with Ova with or without treatment with Dex. The Ova-specific immunoglobulin ELISA was used as technique for the analysis. For Ig detection different sample dilutions were used dependent on the Ig isotype: IgE 1:100, IgG2a 1:10 000; IgG1 1:100 000. The data represent the mean OD +/- SEM deviation. $\left(\mathrm{GR}^{\mathrm{wt}}\right.$ : Control $\mathrm{n}=9$; AAI $n=11$; AAI+Dex $n=11$; GR $^{\text {dim }}$ : Control n=13; AAI n=10; AAI+Dex n=14).

As it can be seen in this analysis there are no differences in Ig production between allergic and Dexamethasone-treated animals irrespective of the genotype. 


\subsubsection{Histology}

In order to compare the inflammation in the lungs of $\mathrm{GR}^{\mathrm{wt}}$ and $\mathrm{GR}^{\mathrm{dim}}$ mice a HE-staining of lung sections was used in addition to the FACS analysis shown before.
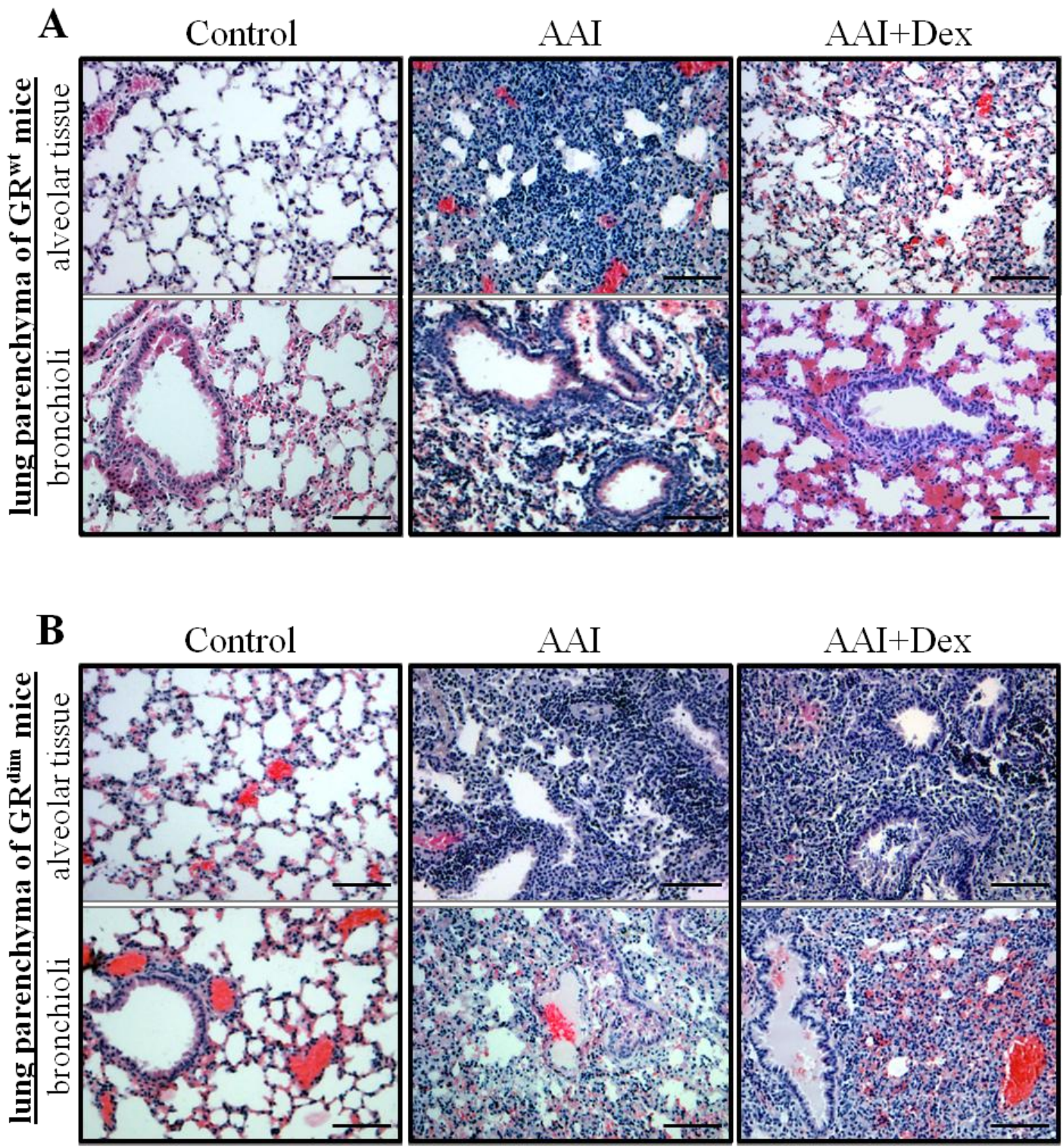

Figure 41: Histological analysis of the lung structure of healthy, AAI and Dex-treated AAI GR ${ }^{\text {wt }}$ (A) and $\mathbf{G R}^{\mathrm{dim}}$ (B) mice. For the histological analysis of structural and cellular changes of lung tissue and effects of GC-therapy on $\mathrm{GR}^{\mathrm{wt}}$ and $\mathrm{GR}^{\mathrm{dim}}$ mice the lungs were collected $40 \mathrm{hrs}$ after the last challenge with Ova. After fixation with PFA the tissue samples were cut in $4 \mu \mathrm{m}$ sections and stained with HE. (A) The pictures show the alveolar tissue and the conducting airways (at 20x magnification) of healthy control, AAI and Dex-treated AAI GR ${ }^{\mathrm{wt}}$ mice. (B) The pictures show lung sections from healthy control and inflammatory lung tissue (at 20x magnification) of AAI and Dex-treated GR dim $^{\text {dim }}$ mice. The scale bars in the lower right corner of all images are equal to $100 \mu \mathrm{m}$.

$\mathrm{GR}^{\mathrm{dim}}$ mice developed a strong inflammation after induction of an asthma-like reaction in the lungs accompanied by massive cell infiltration. The cell influx into the alveolar tissue and the 
accumulation of the cells near the wall of conducting airways in asthmatic animals were comparable between $\mathrm{GR}^{\mathrm{wt}}$ and $\mathrm{GR}^{\mathrm{dim}}$ mice. The airway epithelium showed first remodelling stages with hyperplasia of goblet cells and mucus hypersecretion.

The anti-inflammatory effect of GCs on lung infiltration can only be seen in GR ${ }^{\mathrm{wt}}$ mice whereas the number of infiltrating cells in the lungs of treated $\mathrm{GR}^{\mathrm{dim}}$ mice was not reduced through GC-therapy (Figure 41). The treatment of $\mathrm{GR}^{\mathrm{wt}}$ animals led to an observable effect on the lung sections: the exoneration of bronchioles from mucus and enlargement of free space for gas exchange with normal alveolar tissue reformation. These anti-inflammatory effects were not detectable by histological analysis of the lungs from $\mathrm{GR}^{\mathrm{dim}}$ mice.

\subsubsection{ProSense680 imaging of $G^{\mathrm{wt}}$ and $\mathrm{GR}^{\mathrm{dim}}$ mice}

To investigate inflammation in the lungs of $\mathrm{GR}^{\mathrm{dim}}$ mice after induction of asthma-like reaction the non-invasive method NIR Imaging with the ProSense680 probe was additionally used. In this experimental setup were used the same parameters (time point and concentration) for imaging as previously established with BALB/c wild type mice.

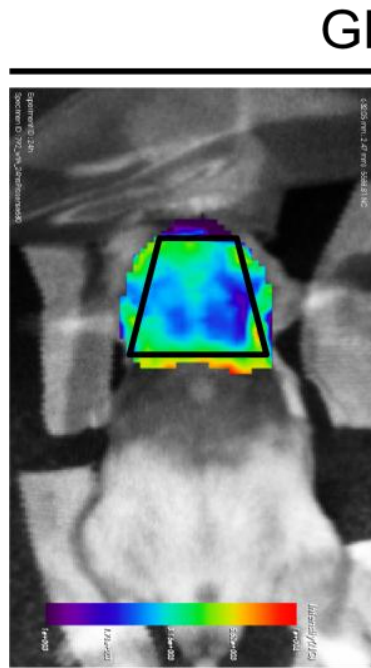

Control
GRwt

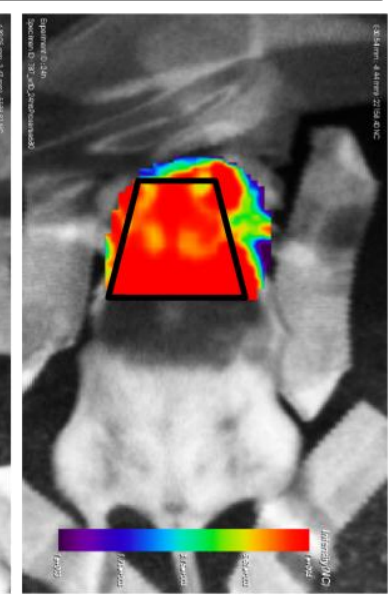

AAI

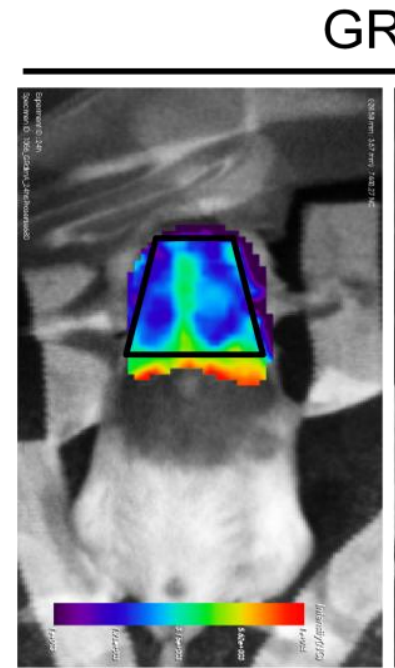

Control
GRdim

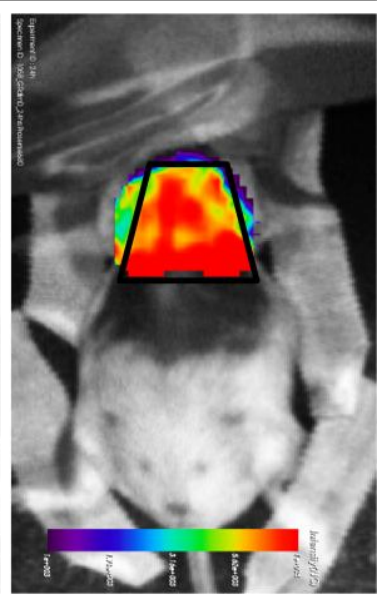

AAI

Figure 42: NIR Imaging of $\mathbf{G R}^{\mathrm{wt}}$ and $\mathbf{G R}^{\mathrm{dim}}$ mice with ProSense680. The fluorescence level of the activated ProSense680 probe was measured $24 \mathrm{hrs}$ after the last challenge of the mice with Ova (20 hrs after ProSense680 dye injection with 5 nmol ProSense680 i.v.). The AAI was induced like described before in Figure 10. The pictures were analysed with OptiView software and show the fluorescence level in the lung region of healthy (control) and AAI mice measured in vivo

Fluorescence scanning of the thoracic region showed a strong induction of the allergic response in the lungs of $\mathrm{GR}^{\mathrm{wt}}$ and $\mathrm{GR}^{\mathrm{dim}}$ mice. Both mouse strains demonstrated very similar ProSense680 activation suggesting a comparable inflammation level. 


\subsubsection{Detection of IL-5 secretion in serum samples of $G^{\mathrm{dim}}$ mice}

Because the maturation and release of eosinophils from the bone marrow is dependent on IL-5 secretion of this cytokine plays an important role in the asthmatic reaction. IL-5 can be produced by $\mathrm{T}_{\mathrm{H}} 2$ cells but also by mast cells and eosinophils themselves. This autocrine mechanism of IL-5 secretion may contribute to the chronic course of allergic inflammation in the lungs with strong eosinophilia.

Because IL-5 is thought to play an important role in asthmatic bronchomucosal inflammation and could be a potential therapeutic target in asthma therapy it was interesting to analyse its concentration in the serum of GC-treated and asthmatic mice.

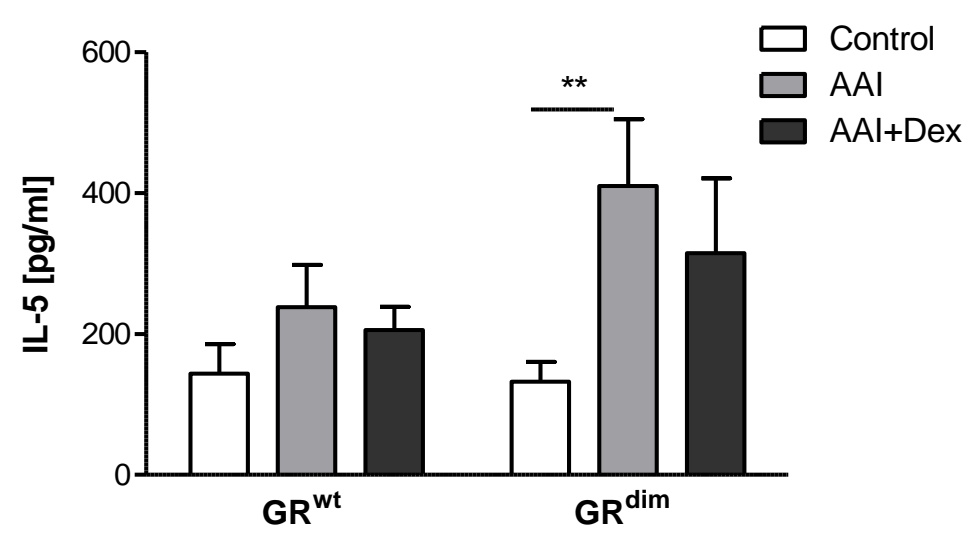

Figure 43: Analysis of the IL-5 concentration in the serum of $\mathbf{G R}^{\mathrm{wt}}$ and $\mathbf{G R}^{\mathrm{dim}}$ mice. The isolated serum samples (from control, AAI and Dex-treated (AAI+Dex) mice) were analyzed by IL-5 ELISA. The data represent the mean concentration $+/$ - SEM $\left(\mathrm{GR}^{\mathrm{wt}}\right.$ : control $n=12$, AAI $n=14$; AAI+Dex $n=19$; $\mathrm{GR}^{\mathrm{dim}}$ : control $\mathrm{n}=11 ; \mathrm{AAI} \mathrm{n}=12 ; \mathrm{AAI}+\operatorname{Dex} \mathrm{n}=11$ )

This experiment demonstrated an increase of IL-5 secretion into the serum of AAI mice of both genotypes. The slightly reduction of IL-5 concentration after Dexamethasone-treatment could be seen in the serum of $\mathrm{GR}^{\mathrm{dim}}$ mice while the level of IL-5 production in $\mathrm{GR}^{\mathrm{wt}}$ mice remained nearly unchanged.

\subsubsection{Effects of GCs on the barrier function of the lung endothelium in GR ${ }^{\text {dim }}$ mice with AAI}

Because the altered microvascular permeability is related to the injury process during an inflammatory reaction this parameter is an important sign of disease progression. To investigate the influence of GC-therapy on the barrier function of the asthmatic lung 
endothelium the lungs of $\mathrm{GR}^{\mathrm{wt}}$ and $\mathrm{GR}^{\mathrm{dim}}$ mice were analyzed using a modified Evans blue extravasation technique.

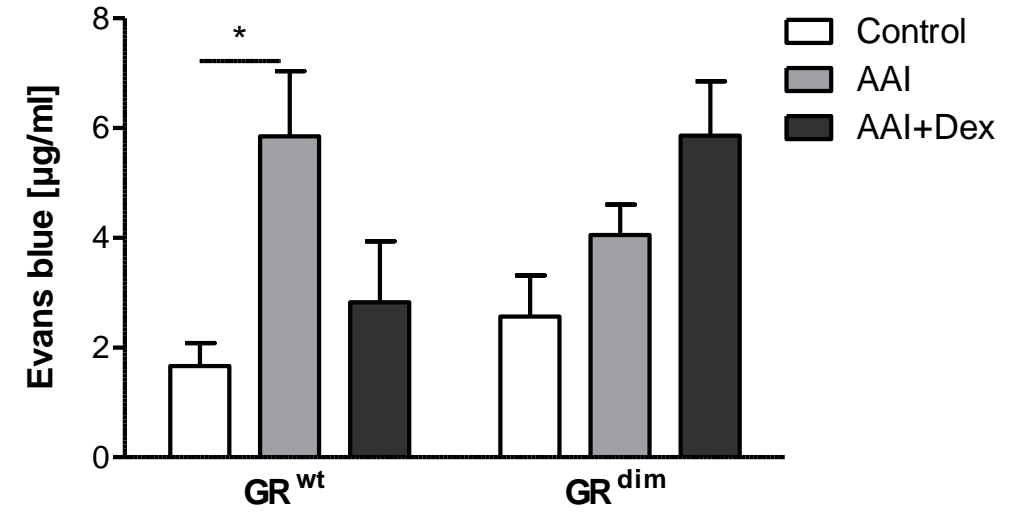

Figure 44: Microvascular permeability test of $\mathbf{G R}^{\mathrm{wt}}$ and $\mathbf{G R}^{\mathrm{dim}}$ mice. The barrier function of the lung endothelium was tested via the Evans blue extravasation technique. Evans blue solution (25 $\mathrm{mg} / \mathrm{kg}$ ) was injected i.v. and circulated for $30 \mathrm{~min}$ through the blood in healthy, AAI and Dextreated AAI mice. Afterwards the Evans blue was extracted from the pulmonary tissues during $24 \mathrm{hrs}$ of incubation at $60{ }^{\circ} \mathrm{C}$ in Formamide. The concentration of Evans blue was determined by spectrophotometric analysis. The data represent the mean of Evans blue concentration $+/$ - SEM (GR ${ }^{\mathrm{wt}}$ : Control n=2; AAI $n=2 ;$ AAI+Dex n=2; $\mathrm{GR}^{\mathrm{dim}}$ : Control $n=2 ;$ AAI n=2; AAI+Dex $n=3$ )

To analyze the microvascular permeability, Evans blue was injected i.v. and after a circulating phase the lungs were dissected and the Evans blue could be extracted from the lung tissue. This analysis showed that the permeability of the endothelium was significantly increased after induction of an asthma-like reaction. While this pathogenic process could be successfully suppressed by GC-therapy in the lungs of $\mathrm{GR}^{\mathrm{wt}}$ mice the barrier function of $\mathrm{GR}^{\mathrm{dim}}$ mice could be not improved by Dexamethasone treatment.

\subsubsection{Gene expression analysis of the BAL and Lung in $\mathrm{GR}^{\mathrm{dim}}$ mice}

The GR is known to modulate gene transcription via DNA-binding-dependent (transactivation) and DNA-binding independent (transrepression) mechanisms.

To better understand the effects of GC-therapy on the gene expression the samples were analyzed via RT-qPCR. The relative gene expression of each group was normalized to the mean gene expression of asthmatic mice of each genotype. As a consequnce of the allergic response in the lungs after induction of an asthma-like reaction the gene expression of a variety of cytokines and chemokines is altered. The expression of most of them is strongly increased in AAI animals. In order to analyze the differences in the regulation of gene expression between Dexamethasone-treated $\mathrm{GR}^{\mathrm{wt}}$ and $\mathrm{GR}^{\mathrm{dim}}$ mice RT-qPCR analysis was used. To investigate the effects of GCs on the infiltrating cells and the lung parenchyma separately, the genes of interest were analyzed in the BAL as well as in lung tissue samples. 
The inflammatory response in the lungs led to an increased expression of several inflammatory mediators in BAL and lung tissue on mRNA level.

The first group of analyzed genes served as markers for the cell type specific infiltration and cell migration into the lungs: IL-5, Eotaxin-2, MCP-1, MIP-1a, CXCR3 and CXCR4. Gene expression analysis of these cytokines and chemokines demonstrates an induction of mRNA expression after the development of an asthma-like reaction in the lungs.

IL-5 and Eotaxin-2 play an important role in the development of eosinophilia in the lungs. The expression of IL-5 mRNA was successfully downregulated by GC-therapy in BAL samples of $\mathrm{GR}^{\mathrm{wt}}$ and $\mathrm{GR}^{\mathrm{dim}}$ mice. IL-5 was also downregulated in $\mathrm{GR}^{\mathrm{wt}}$ lungs while the $\mathrm{GR}^{\mathrm{dim}}$ lung cells expressed unchanged level of IL-5.The same effect was seen for Eotaxin-2.
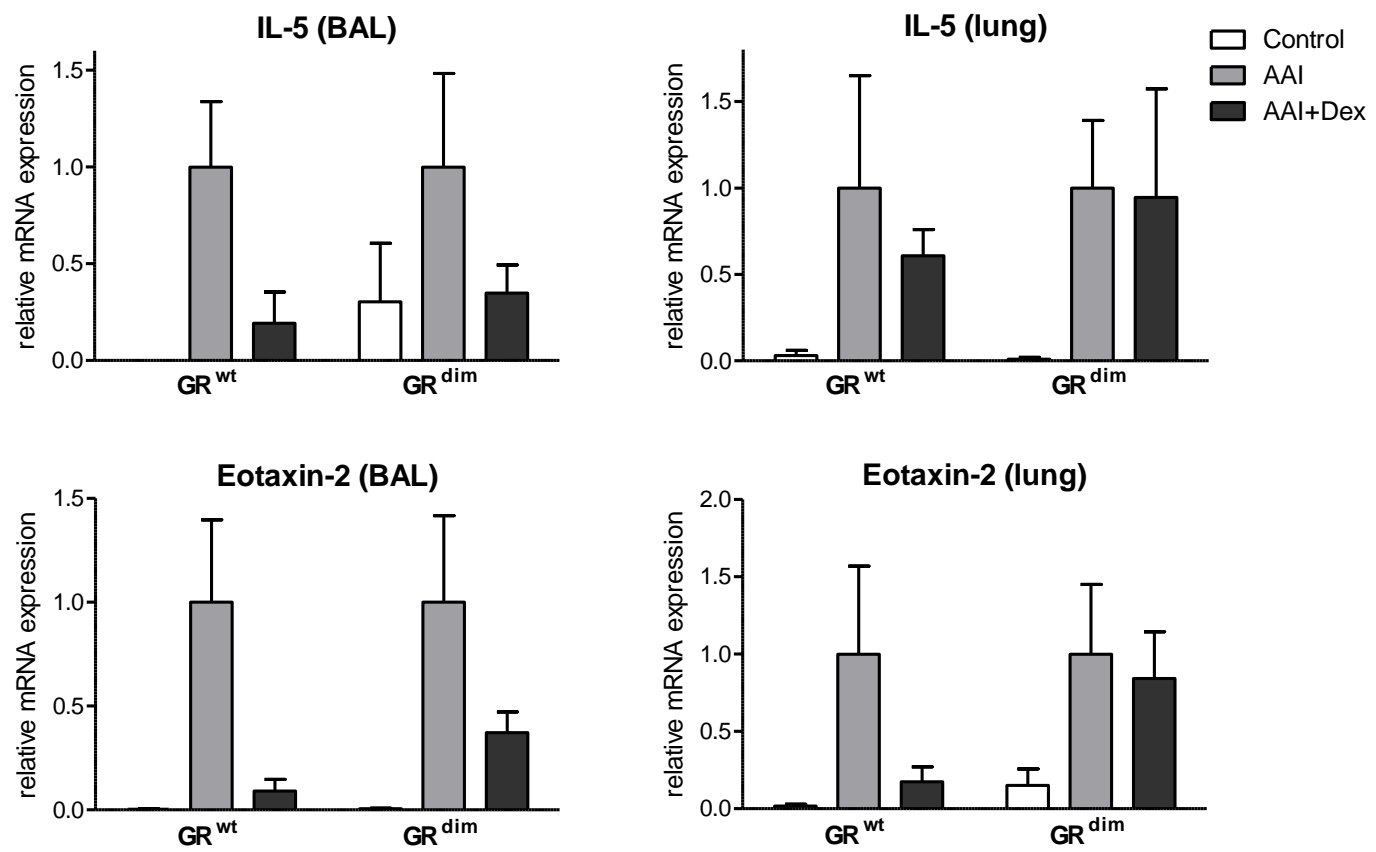

Figure 45: RT-qPCR analysis of IL-5 and Eotaxin2 mRNA expression in lung tissue and BAL samples of $\mathbf{G R}^{\mathrm{wt}}$ and $\mathbf{G R}^{\mathrm{dim}}$ mice. BAL samples and lung tissue were isolated from healthy (control), $\mathrm{AAI}$ and Dex-treated AAI GR ${ }^{\mathrm{wt}}$ and $\mathrm{GR}^{\mathrm{dim}}$ mice. The AAI induction and Dex-treatment was done as described before in Figure 29. The data represent the mean mRNA expression normalized to samples of AAI mice +/- SEM (GR ${ }^{\mathrm{wt}}$ : control $n=2$; AAI $n=2$; AAI+Dex $n=2$; $\mathrm{GR}^{\mathrm{dim}}$ : control $n=2$; AAI $n=3$; $\mathrm{AAI}+\operatorname{Dex} \mathrm{n}=3$ ) 
CXCR3 is preferentially expressed on activated $\mathrm{T}_{\mathrm{H}} 1, \mathrm{NK}$ and some epithelial and endothelial cells. Its expression is important for lymphocyte migration into the lungs and its ligands can block the migration of $\mathrm{T}_{\mathrm{H}} 2$ cells and effector $\mathrm{T}$ cells in response to CCR3. CXCR3 expression was only lightly upregulated after induction of an asthma-like reaction in the infiltrating cells. In the lungs the expression was strongly upregulated and could be suppressed by GCtreatment of $\mathrm{GR}^{\mathrm{wt}}$ and $\mathrm{GR}^{\mathrm{dim}}$ mice.

CXCR4 mobilizes hematopoietic stem cells into the bloodstream during an inflammatory response. The data from BAL cell samples show CXCR4 expression was not significantly increased or suppressed in any experimental group. While all BAL samples showed comparable CXCR4 expression, the induction of an asthma-like reaction led to an increased expression of this gene in lung tissue samples of $\mathrm{GR}^{\mathrm{wt}}$ mice. GC-therapy did not change CXCR4 mRNA expression irrespective of the genotype.
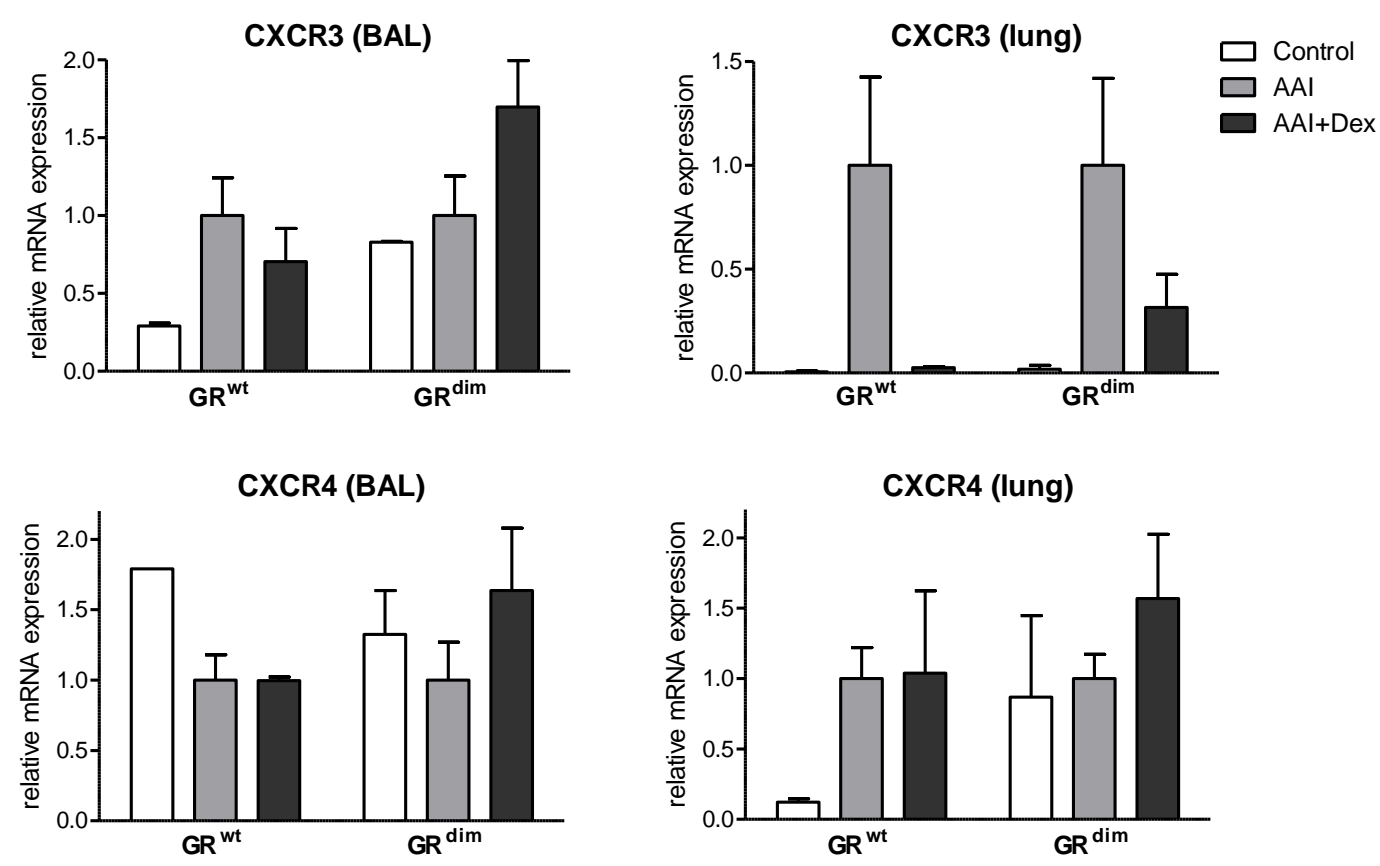

Figure 46: The RT-qPCR analysis of CXCR3 and CXCR4 mRNA expression in lung tissue and BAL samples of $\mathbf{G R}^{\mathrm{wt}}$ and $\mathbf{G R}^{\mathrm{dim}}$ mice. BAL samples and lung tissue were isolated from healthy (control), AAI and Dex-treated AAI GR ${ }^{\mathrm{wt}}$ and $\mathrm{GR}^{\mathrm{dim}}$ mice. The AAI induction and Dex-treatment was done as described before in Figure 29. The data represent the mean mRNA expression normalized on samples of AAI mice $+/$ - SEM $\left(\mathrm{GR}^{\mathrm{wt}}\right.$ : control $n=2$; AAI $n=2$; AAI + Dex $n=2 ; \mathrm{GR}^{\mathrm{dim}}$; control $n=2$; AAI $\mathrm{n}=3$; AAI+Dex $n=3$ ) 
To investigate inflammatory mediators which play a role in macrophage and monocyte migration and activation, MCP-1 and MIP-1 $\alpha$ were analyzed.
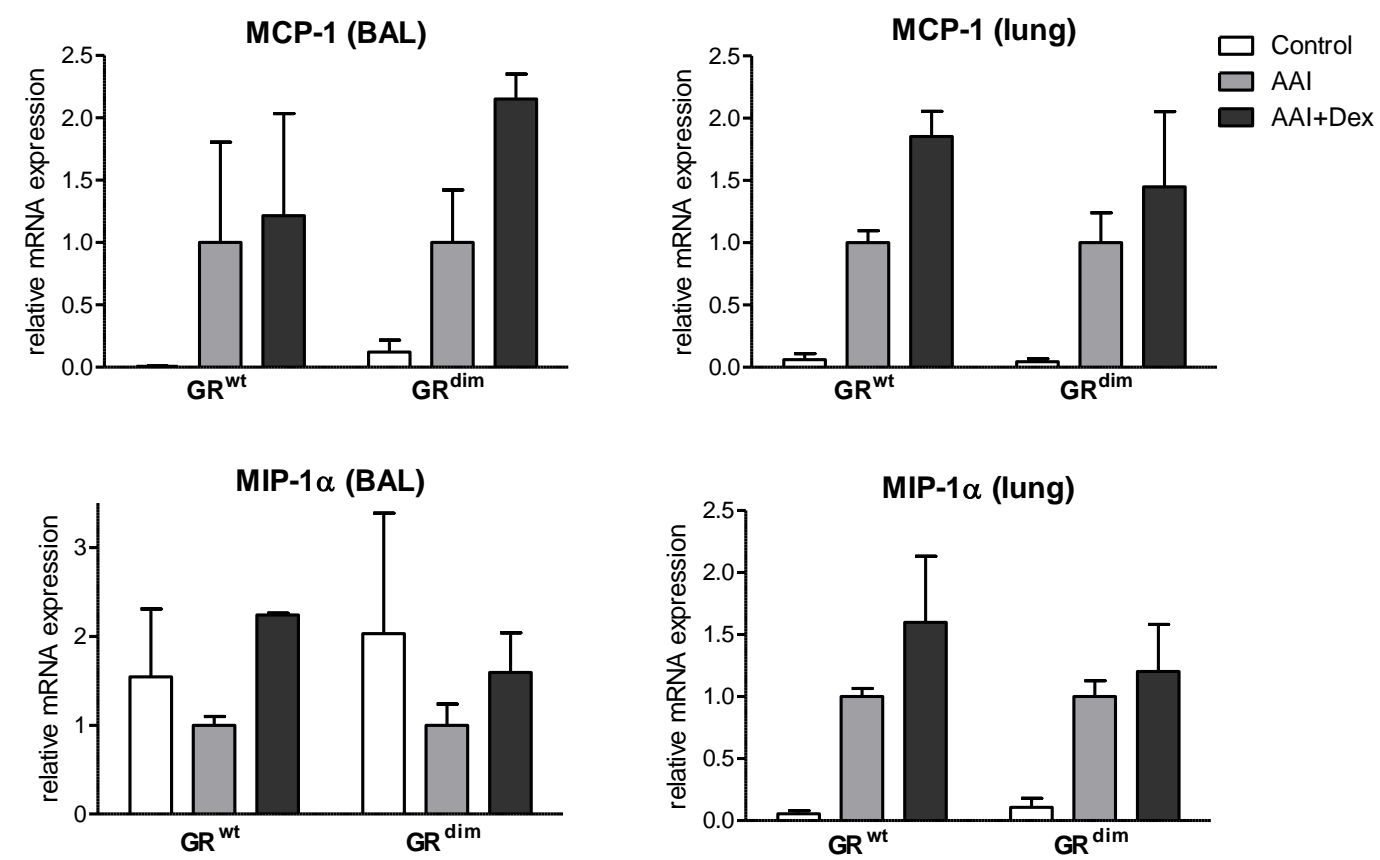

Figure 47: The RT-qPCR analysis of MCP-1 and MIP-1a mRNA expression in lung tissue and BAL samples of $\mathbf{G R}^{\mathrm{wt}}$ and $\mathbf{G R}^{\mathrm{dim}}$ mice. On the analysis day the BAL samples and lung tissue were isolated from healthy (control), AAI and Dex-treated AAI GR ${ }^{\mathrm{wt}}$ and $\mathrm{GR}^{\mathrm{dim}}$ mice. The AAI induction and Dex-treatment was done as described before in Figure 29. The data represent the mean mRNA expression normalized on samples of AAI mice $+/$ - SEM $\left(\mathrm{GR}^{\mathrm{wt}}\right.$ : control $\mathrm{n}=2$; AAI $n=2$; AAI+Dex $\mathrm{n}=2 ; \mathrm{GR}^{\mathrm{dim}}$ : control $\mathrm{n}=2 ;$ AAI $\mathrm{n}=3 ; \mathrm{AAI}+\operatorname{Dex} \mathrm{n}=3$ )

It was reported that alveolar macrophages produced increased amount of these two cytokines in asthmatic lungs. The analysis of BAL samples demonstrated a strong increase in MCP-1 expression in $\mathrm{GR}^{\mathrm{wt}}$ and $\mathrm{GR}^{\mathrm{dim}}$ mice with an allergic reaction and nearly unchanged expression of MIP-1a. After GC-therapy MCP-1 and MIP-1 $\alpha$ expression were slightly induced in BAL samples. The expression of these cytokines was strongly induced in the lungs of asthmatic animals. Treatment with Dexamethasone led to a further increase of MCP-1 and MIP-1 $\alpha$ in lung tissue. 
Other analyzed genes belong to a group of genes that impact inflammation: IL-4, IL-13, RANTES and IL-10.
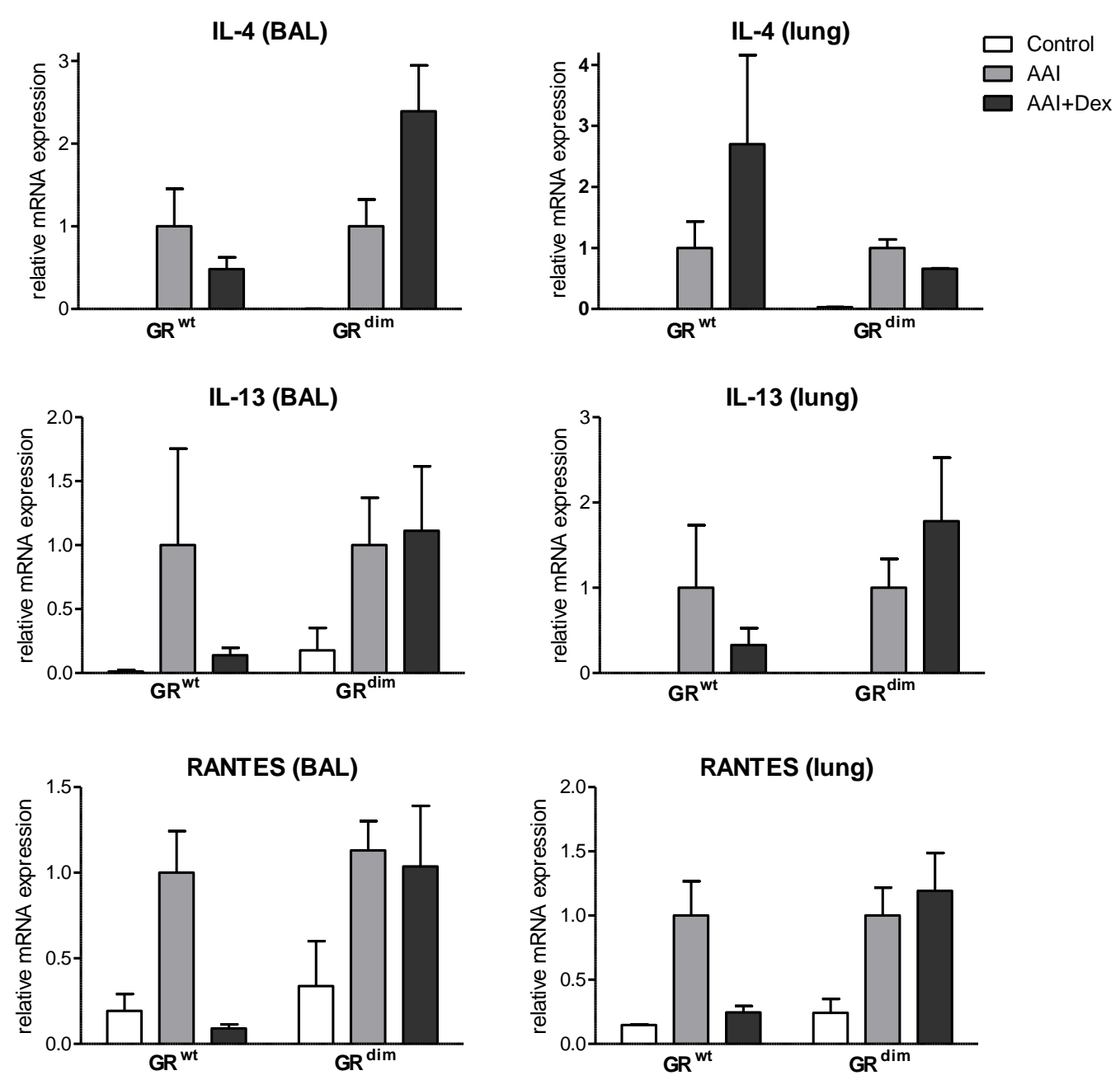

Figure 48: RT-qPCR analysis of IL-4, IL-13 and RANTES mRNA expression in lung tissue and BAL samples of $\mathbf{G R}^{\mathrm{wt}}$ and $\mathbf{G R}^{\mathrm{dim}}$ mice. BAL samples and lung tissue were isolated from healthy (control), AAI and Dex-treated AAI GR ${ }^{\mathrm{wt}}$ and $\mathrm{GR}^{\mathrm{dim}}$ mice. The AAI induction and Dex-treatment was done as described before in Figure 29. The data represent the mean mRNA expression normalized on samples of AAI mice $+/$ - SEM $\left(\mathrm{GR}^{\mathrm{wt}}\right.$ : control $n=2$; AAI $n=2$; AAI+Dex $n=2 ; \mathrm{GR}^{\mathrm{dim}}$ : control $n=2$; AAI $\mathrm{n}=3$; AAI + Dex $n=3$ )

The $\mathrm{T}_{\mathrm{H}} 2$ cytokines IL-4 and IL-13 play a key role in disease progression by activating and recruiting a variety of inflammatory cells such as eosinophils, mast cells and B cells into the lungs. These cytokines were strongly induced in the BAL and lung samples of asthmatic animals of both genotypes. The GC-therapy led to different effects on IL-4 and IL-13 expression: While IL-4 mRNA expression in BAL samples of $\mathrm{GR}^{\mathrm{wt}}$ mice was downregulated the expression of this cytokine in the lung was elevated. The opposite effect was observed in samples of GR ${ }^{\text {dim }}$ mice: IL-4 expression was elevated in BAL samples of GC-treated mice while the expression in lung remained unaltered. 
RANTES leads to increased eosinophilia independent on the presence of the allergen. The mRNA expression data demonstrate a strong suppression of RANTES expression after GCtherapy in samples of AAI GR ${ }^{\mathrm{wt}}$ mice. In contrast, GC-therapy of $\mathrm{GR}^{\mathrm{dim}}$ mice did not affect the RANTES mRNA expression in BAL or lung tissue samples.
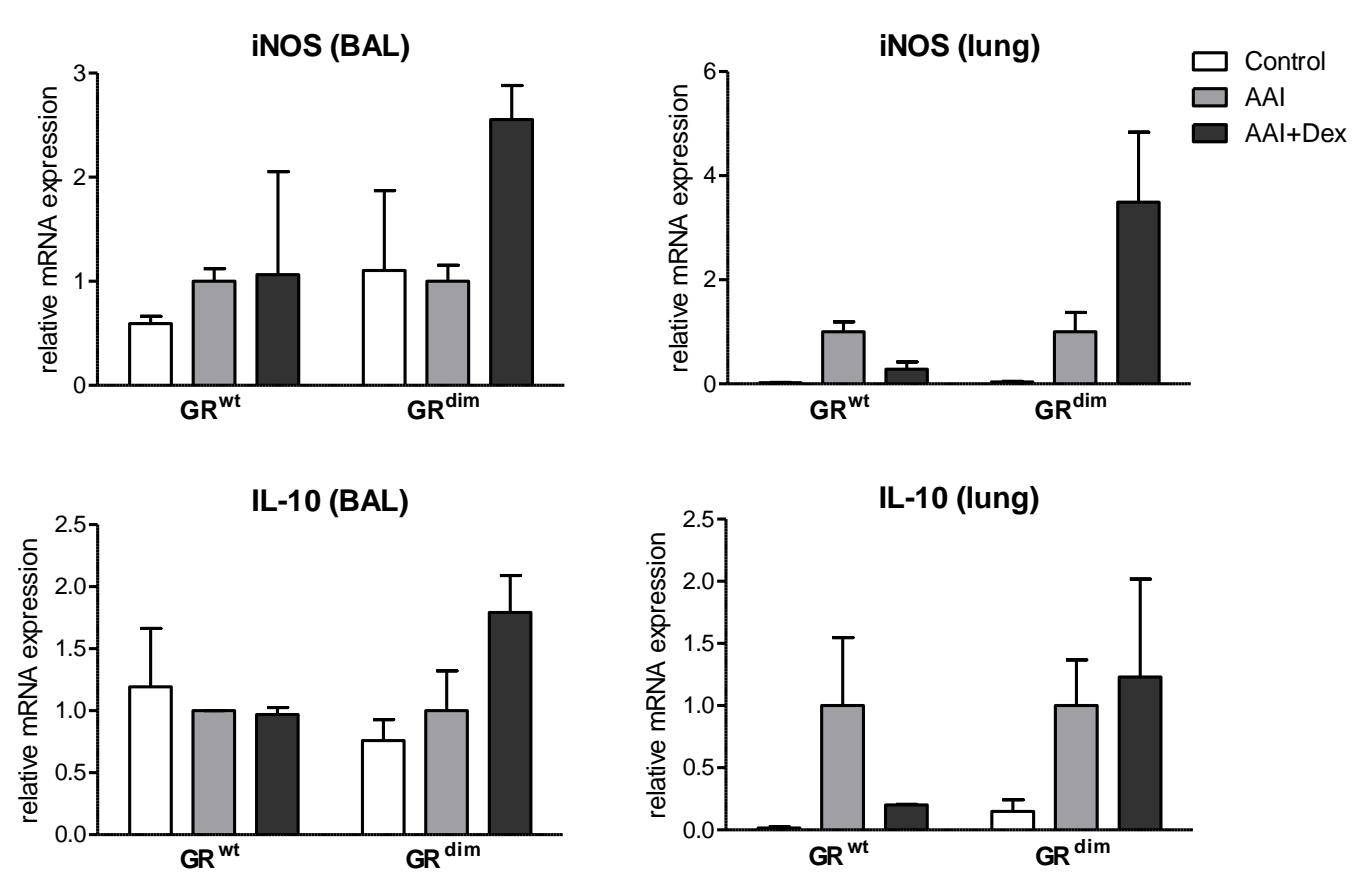

Figure 49: RT-qPCR analysis of iNOS and IL-10 mRNA expression in lung tissue and BAL samples of $\mathbf{G R}^{\mathrm{wt}}$ and $\mathbf{G R}^{\mathrm{dim}}$ mice. BAL samples and lung tissue were isolated from healthy (control), $\mathrm{AAI}$ and Dex-treated AAI GR ${ }^{\mathrm{wt}}$ and $\mathrm{GR}^{\mathrm{dim}}$ mice. The AAI induction and Dex-treatment was done as described before in Figure 29. The data represent the mean mRNA expression normalized on samples of AAI mice +/-SEM $\left(\mathrm{GR}^{\mathrm{wt}}\right.$ : control $n=2$; AAI $n=2 ;$ AAI+Dex $n=2 ; \mathrm{GR}^{\mathrm{dim}}$ : control $n=2 ;$ AAI $n=3$; AAI+Dex $n=3$ )

The inflammatory enzyme iNOS is a characteristic marker of the inflamed epithelium and activated macrophages. The results of the iNOS gene expression analysis demonstrated that the allergic response in the lungs led to only a minor increase in iNOS expression in the infiltrating cells but the induction of an asthma-like reaction caused a significant upregulation of iNOS expression in lung tissue of both genotypes. The therapy with Dexamethasone suppressed iNOS expression in the lung cells of $\mathrm{GR}^{\mathrm{wt}}$ mice while the expression of this inflammatory enzyme was up-regulated in GC-treated asthmatic $\mathrm{GR}^{\mathrm{dim}}$ mice.

IL-10 was shown to play a conflicting role in disease development and progression. While this cytokine is an important anti-inflammatory cytokine, the over-expression of this mediator can lead to the development of fibrosis in asthmatic lungs. IL-10 expression was unchanged in the BAL samples of $\mathrm{GR}^{\mathrm{wt}}$ mice while the samples of asthmatic $\mathrm{GR}^{\mathrm{dim}}$ mice demonstrated a 
slightly induction of IL-10 expression after the GC-treatment. In contrast, the expression of this cytokine was strongly induced in lung tissue of $\mathrm{GR}^{\mathrm{wt}}$ and $\mathrm{GR}^{\mathrm{dim}}$ mice. This elevated expression of IL-10 after induction of an asthma-like reaction could be strongly suppressed in $\mathrm{GR}^{\mathrm{wt}}$ mice by Dexamethasone treatment while the IL-10 mRNA expression in the lungs of GC-treated GR ${ }^{\text {dim }}$ mice was unchanged. 


\subsection{The role of non-hematopoietic cell types in the therapy of asthma with GCs}

\subsubsection{Bone marrow chimeras}

To investigate the role of non-immune cells in the treatment of asthmatic reactions bone marrow chimeras were used. $\mathrm{GR}^{\mathrm{wt}}$ or $\mathrm{GR}^{\mathrm{dim}}$ mice served as recipients and were reconstituted with either $\mathrm{GR}^{\mathrm{wt}}$ or $\mathrm{GR}^{\mathrm{dim}}$ bone marrow. This resulted in four experimental groups in total.
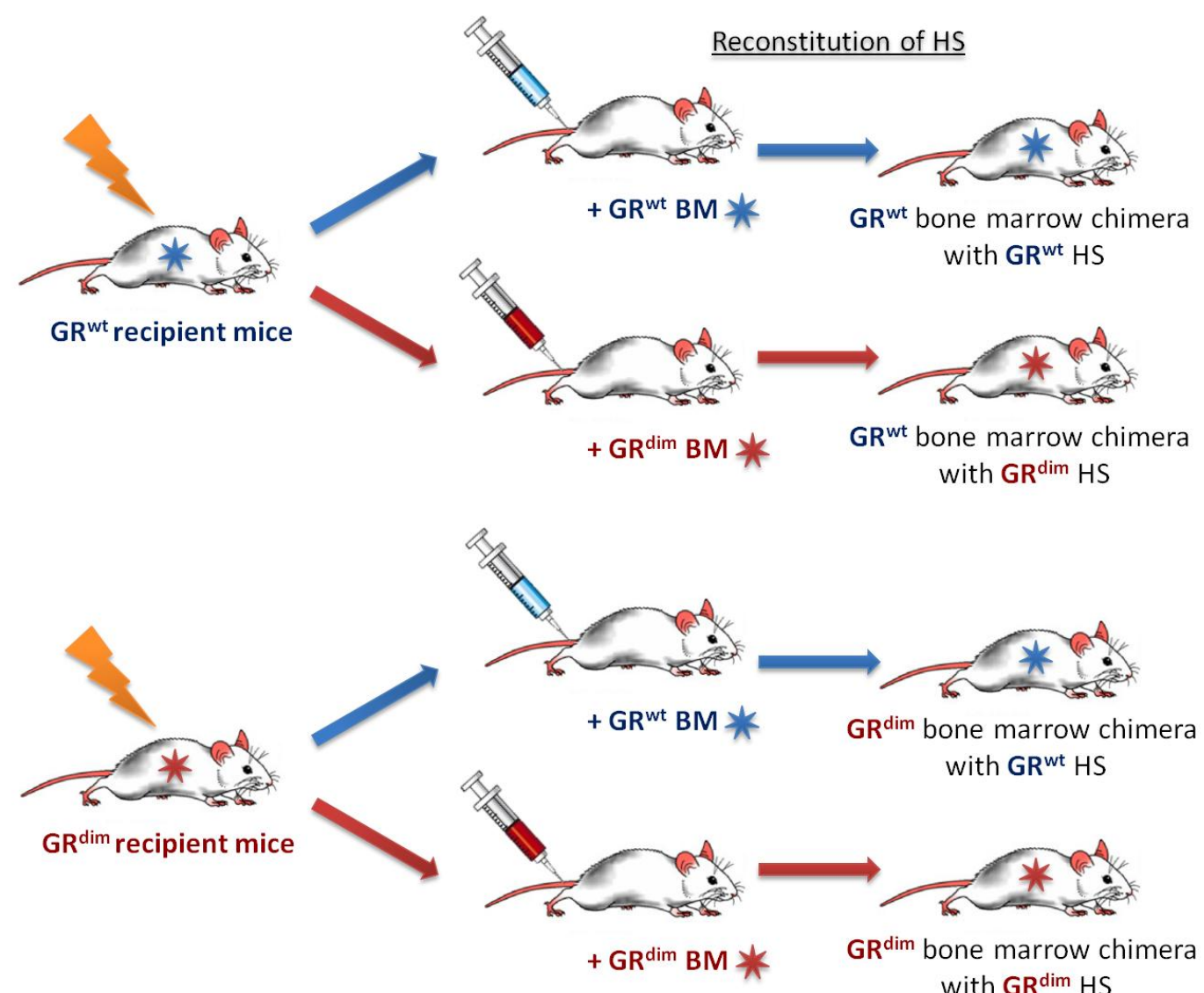

Figure 50: Reconstitution schema of hematopoietic system. $\mathrm{GR}^{\mathrm{wt}}$ and $\mathrm{GR}^{\mathrm{dim}}$ recipient mice at the age of 8-10 weeks were lethally irradiated with $8.5 \mathrm{~Gy}$ at a rate of $1 \mathrm{~Gy}$ per minute to delete their own hematopoietic cells. On the next day mice were reconstituted with bone marrow from $\mathrm{GR}^{\mathrm{wt}}$ or $\mathrm{GR}^{\mathrm{dim}}$ donor mice. After 6 weeks the reconstitution efficiency of hematopoietic system (HS) was tested. The obtained bone marrow chimeras were: $\mathrm{GR}^{\mathrm{wt}}$ with $\mathrm{GR}^{\mathrm{wt}} \mathrm{HS}, \mathrm{GR}^{\mathrm{wt}}$ with $\mathrm{GR}^{\mathrm{dim}} \mathrm{HS}$ and $\mathrm{GR}^{\mathrm{dim}}$ with $\mathrm{GR}^{\mathrm{wt}} \mathrm{HS}, \mathrm{GR}^{\mathrm{dim}}$ with $\mathrm{GR}^{\mathrm{dim}} \mathrm{HS}$. After complete reconstitution of the haematopoietic system the AAI was induced as described before. 


\subsubsection{BAL analysis}

First of all, the cell number of the BAL was determined.

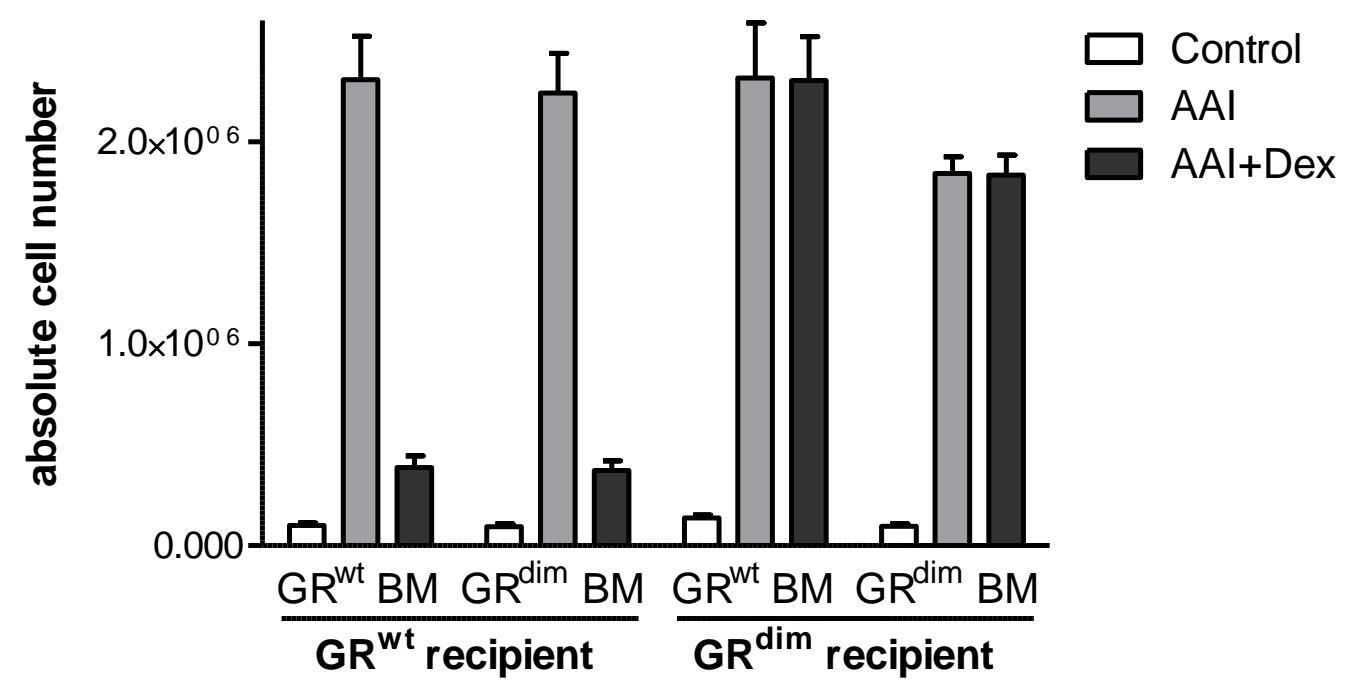

Figure 51: Absolute cell numbers in the BAL of healthy (control), AAI and GC-treated (AAI+Dex) $G^{\mathrm{wt}}$ and $\mathbf{G R}^{\mathrm{dim}}$ bone marrow chimeras. $\mathrm{GR}^{\mathrm{wt}}$ and $\mathrm{GR}^{\mathrm{dim}}$ recipient mice were reconstituted with bone marrow from $\mathrm{GR}^{\mathrm{wt}}$ or $\mathrm{GR}^{\mathrm{dim}}$ donor mice. After complete reconstitution of the haematopoietic system the AAI was induced and the BAL was isolated $40 \mathrm{hrs}$ after the last Ovachallenge. The absolute cell number in the BAL was determined with a Neubauer cell count chamber. The data represent the mean cell number $+/$ - SEM from four separate experiments $\left(\mathrm{GR}^{\mathrm{wt}}\right.$ recipient with $\mathrm{GR}^{\mathrm{wt}}$ BM: Control $\mathrm{n}=6$; AAI $\mathrm{n}=7$; $\mathrm{AAI}+\mathrm{Dex} \mathrm{n}=8$; $\mathrm{GR}^{\mathrm{wt}}$ recipient with $\mathrm{GR}^{\mathrm{dim}} \mathrm{BM}$ : Control $\mathrm{n}=5$; AAI $\mathrm{n}=8$; AAI+Dex $n=8 ; \mathrm{GR}^{\mathrm{dim}}$ recipient: with $\mathrm{GR}^{\mathrm{wt}} \mathrm{BM}$ : Control $\mathrm{n}=7$; AAI $\mathrm{n}=8$; AAI+Dex $\mathrm{n}=11$; $\mathrm{GR}^{\mathrm{dim}}$ recipient with $\mathrm{GR}^{\mathrm{dim}} \mathrm{BM}$ : Control $\mathrm{n}=3$; $\mathrm{AAI} n=3$; AAI+Dex $n=3$ )

The irradiation of the recipient mice did not influence the induction of an asthma-like reaction in $\mathrm{GR}^{\mathrm{wt}}$ and $\mathrm{GR}^{\mathrm{dim}}$ bone marrow chimeras. Without immunization and sensitization with Ova the mice did not develop an inflammatory response and showed normal cell numbers in the BAL.

After induction of an asthma-like reaction the $\mathrm{GR}^{\mathrm{wt}}$ and $\mathrm{GR}^{\mathrm{dim}}$ bone marrow chimeras all showed very similar lung infiltration with about $2 \times 10^{6}$ cells in the asthmatic BAL. The reduction of the cell number after the GC-therapy was only dependent on the genotype of the recipient mice and did not show any correlation with the genotype of the donor cells. 


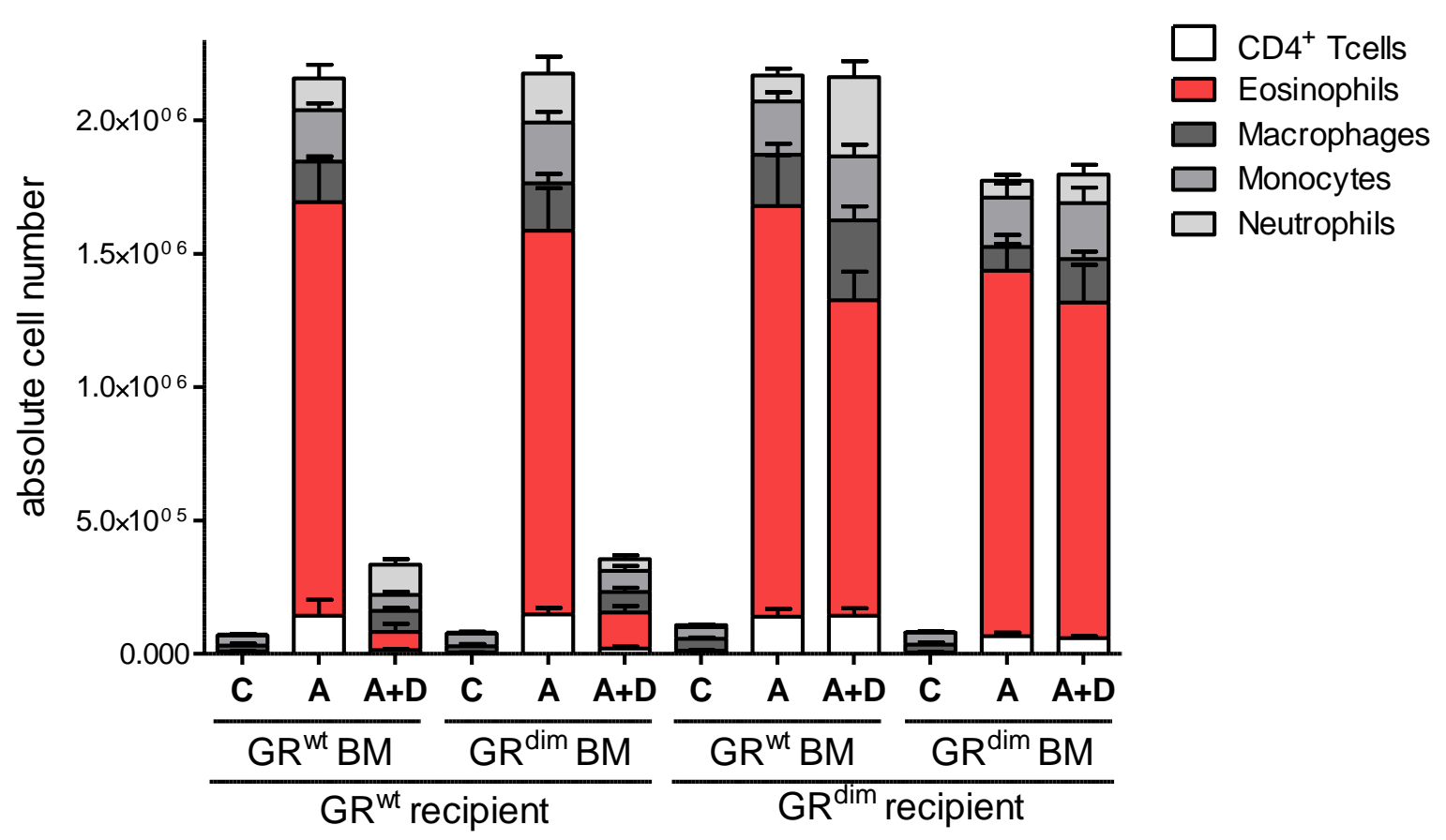

Figure 52: Analysis of absolute cell numbers of the different subpopulations in BAL samples of $\mathbf{G R}^{\mathrm{wt}}$ and $\mathbf{G R}^{\mathrm{dim}}$ bone marrow chimeras. The relative percentages of $\mathrm{T}$ helper cells, eosinophils, macrophages and monocytes were determined by FACS analysis. Based on this relative percentage of the subpopulations and the absolute cell number of the BAL, the absolute cell number of each subpopulation could be calculated. The data represent the mean of the cell numbers +/- SEM from four separate experiments $\left(\mathrm{GR}^{\mathrm{wt}}\right.$ recipient with $\mathrm{GR}^{\mathrm{wt}} \mathrm{BM}$ : Control $\mathrm{n}=6$; AAI $n=7$; $\mathrm{AAI}+\mathrm{Dex} \mathrm{n}=8$; $\mathrm{GR}^{\mathrm{wt}}$ recipient with $\mathrm{GR}^{\mathrm{dim}} \mathrm{BM}$ : Control $\mathrm{n}=5$; $\mathrm{AAI} n=8$; $\mathrm{AAI}+\mathrm{Dex} n=8$; $\mathrm{GR}^{\mathrm{dim}}$ recipient: with $\mathrm{GR}^{\mathrm{wt}} \mathrm{BM}$ : Control $n=7$; AAI $n=8$; AAI+Dex $n=11 ;$ GR $^{\text {dim }}$ recipient with GR $^{\text {dim }}$ BM: Control $n=3$; AAI $n=3$; $\mathrm{AAI}+\operatorname{Dex} \mathrm{n}=3$ )

The cellular infiltration of the lungs with eosinophils could be successfully suppressed by GC-therapy of bone marrow chimeras with $\mathrm{GR}^{\mathrm{wt}}$ mice as recipient. This GC-effect was independent of the genotype of the hematopoietic system. The subpopulations of the BAL of $\mathrm{GR}^{\mathrm{wt}}$ recipients with a $\mathrm{GR}^{\mathrm{wt}}$ or $\mathrm{GR}^{\mathrm{dim}}$ immune system were largely similar. While $\mathrm{GR}^{\mathrm{wt}}$ bone marrow chimeras were fully treatable with Dexamethasone, $\mathrm{GR}^{\mathrm{dim}}$ bone marrow chimeras showed unaltered infiltration of the lungs and no influence of GC-therapy independent of the genotype of the immune cells.

\subsubsection{Proliferation and priming of splenocytes from $G R^{\mathrm{wt}}$ and $G R^{\mathrm{dim}}$ chimeras after antigen-specific restimulation $e x$ vivo}

To investigate the effects of GC-treatment on the proliferation of antigen-specific $\mathrm{T}$ and $\mathrm{B}$ cells restimulation of splenocytes with Ova ex vivo was done. The isolated cells were cultured for 72 hours with and without Ova. IL-2 was analysed by ELISA, proliferation by ${ }^{3} \mathrm{H}-$ thymidine incorporation assay. 


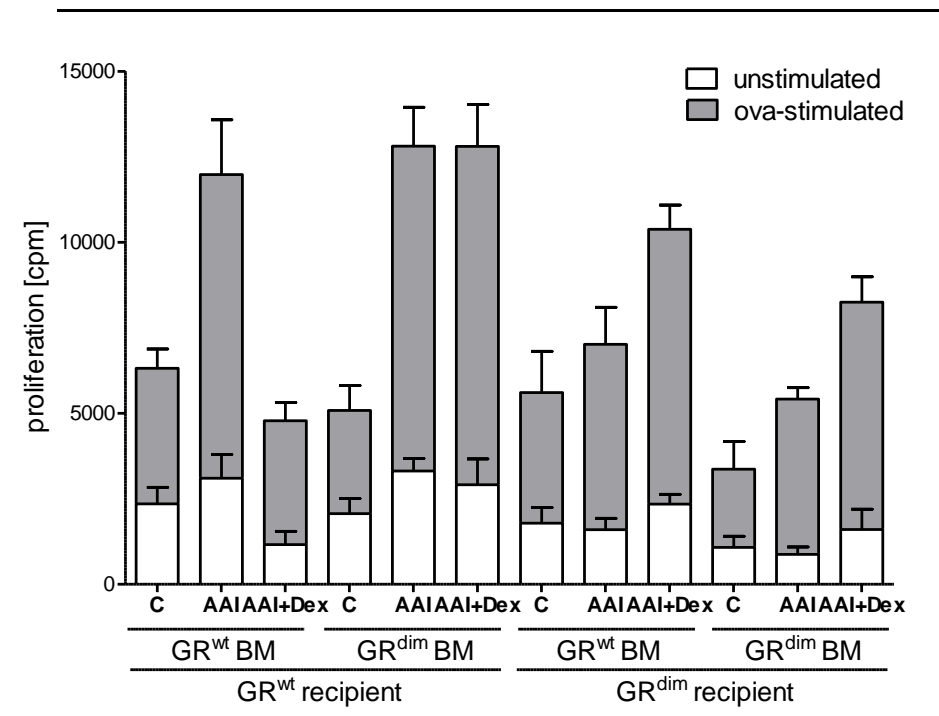

Figure 53: Proliferation and priming of splenocytes from $\mathbf{G R}^{\mathrm{wt}}$ and $\mathbf{G R}^{\mathrm{dim}}$ chimeras after antigenspecific restimulation ex vivo. Proliferation of splenocytes from healthy control (C), AAI and GCtreated (AAI+Dex) mice was determined after $72 \mathrm{hrs}$ incubation with antigen specific restimulation (Ova), compared to the basal proliferation (unstimulated cells). The cells were analysed for their proliferation activity with an ${ }^{3} \mathrm{H}$ thymidine incorporation assay. The

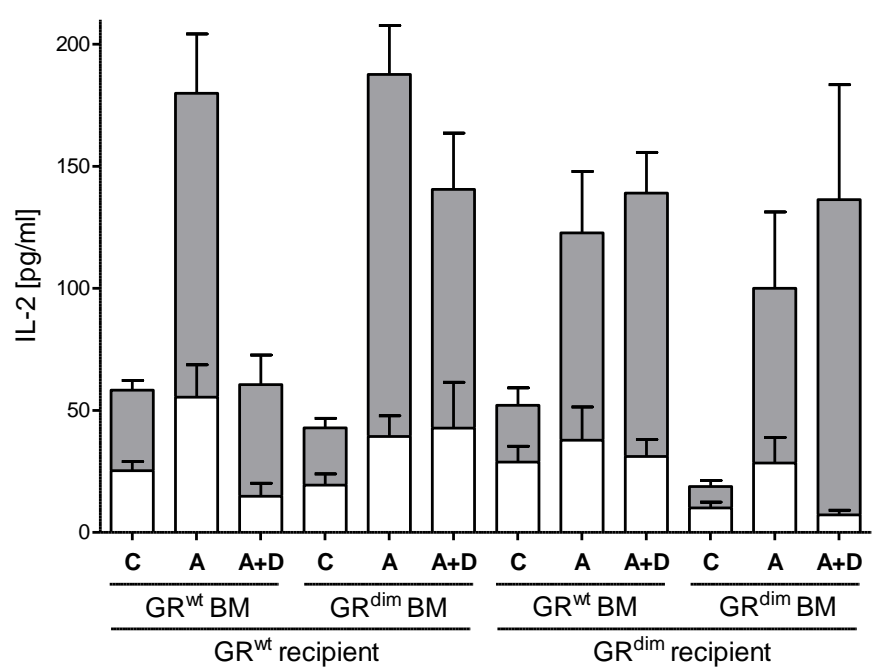
IL-2 release was determined by ELISA. The results show the mean proliferation activity (cpm) or the cytokine concentration +/- SEM of triplicate measurements of 4 separate experiments. $\left(\mathrm{GR}^{\mathrm{wt}}\right.$ recipient with $\mathrm{GR}^{\mathrm{wt}}$ BM: Control $\mathrm{n}=6$; AAI $\mathrm{n}=7$; $\mathrm{AAI}+\mathrm{Dex} \mathrm{n}=8 ; \mathrm{GR}^{\mathrm{wt}}$ recipient with $\mathrm{GR}^{\mathrm{dim}} \mathrm{BM}$ : Control $\mathrm{n}=5$; AAI $\mathrm{n}=8$; AAI+Dex $n=8 ; \mathrm{GR}^{\mathrm{dim}}$ recipient: with $\mathrm{GR}^{\mathrm{wt}}$ BM: Control $\mathrm{n}=7$; AAI $\mathrm{n}=8$; AAI+Dex $n=11 ; \mathrm{GR}^{\mathrm{dim}}$ recipient with GR $^{\operatorname{dim}}$ BM: Control $n=3$; AAI $n=3$; AAI+Dex $n=3$ )

The proliferation test demonstrated that the restimulation of splenocytes with Ova led to an increased proliferation of all types of chimeras. $\mathrm{GR}^{\mathrm{wt}}$ chimeras showed a stronger proliferation as $\mathrm{GR}^{\mathrm{dim}}$ restimulated splenocytes in AAI mice. The treatment of animals with GCs influenced proliferation activity only in $\mathrm{GR}^{\mathrm{wt}}$ chimeras. While the proliferation of splenocytes from $\mathrm{GR}^{\mathrm{wt}}$ chimera with $\mathrm{GR}^{\mathrm{wt}}$ immune system could be significantly suppressed via GCtherapy, the proliferation of $\mathrm{GR}^{\mathrm{wt}}$ chimera with $\mathrm{GR}^{\mathrm{dim}}$ immune system was not downregulated in GC-treated animals. The GR ${ }^{\mathrm{dim}}$ chimeras demonstrated a slightly increased proliferation in GC-treated animals with both type of GR-expression of immune cells. Thus, proliferation is only suppressed if both the immune system and the recipient are GR ${ }^{\mathrm{wt}}$. The IL-2 secretion confirmed the observation from the ${ }^{3} \mathrm{H}$-thymidine incorporation assay. The produced level on IL-2 was strongly increased in supernatants of splenocytes from asthmatic mice, but the concentration of this cytokine was only reduced in the samples of $\mathrm{GR}^{\mathrm{wt}}$ chimeras reconstituted with bone marrow from $\mathrm{GR}^{\mathrm{wt}}$ mice. No reliable results were obtained for IL- 4 and IFN- 8 secretion. 


\subsubsection{Immunoglobulin production in $G^{\mathrm{wt}}$ and $\mathrm{GR}^{\mathrm{dim}}$ bone marrow chimeras}

After induction of an asthma-like reaction in $\mathrm{GR}^{\mathrm{wt}}$ and $\mathrm{GR}^{\mathrm{dim}}$ chimeras the Ig level were significantly increased. The treatment of the asthmatic mice with Dexamethasone did not suppress this elevated immunoglobulin concentration in serum in any type of chimeras.
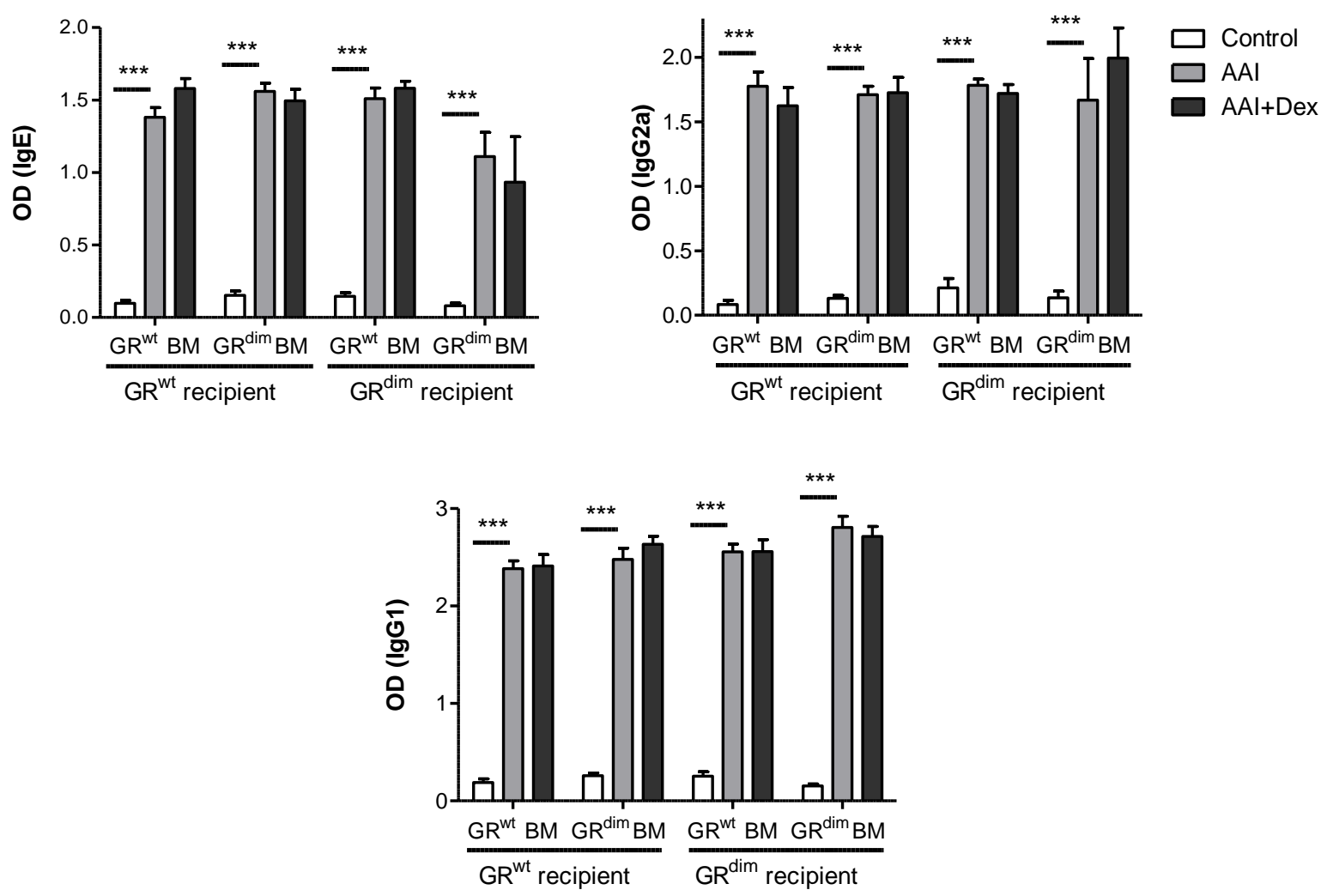

Figure 54: Immunoglobulin isotype production of $\mathbf{G R}^{\mathrm{wt}}$ and $\mathbf{G R}^{\mathrm{dim}}$ bone marrow chimera. The isolated serum from $\mathrm{GR}^{\mathrm{wt}}$ and $\mathrm{GR}^{\mathrm{dim}}$ chimera was analyzed via Ova-specific Ig-ELISA. The samples were diluted dependent on Ig isotype: IgE 1:100, IgG2a 1:1000, IgG1 1:100 000. The data represent the mean OD +/- SEM from 4 separate experiments $\left(\mathrm{GR}^{\mathrm{wt}}\right.$ recipient with $\mathrm{GR}^{\mathrm{wt}} \mathrm{BM}$ : Control $\mathrm{n}=6$; AAI $\mathrm{n}=7$; AAI+Dex $n=8$; $\mathrm{GR}^{\mathrm{wt}}$ recipient with $\mathrm{GR}^{\mathrm{dim}} \mathrm{BM}$ : Control $\mathrm{n}=5$; AAI $\mathrm{n}=8$; AAI+Dex $\mathrm{n}=8$; $\mathrm{GR}^{\mathrm{dim}}$ recipient: with $\mathrm{GR}^{\mathrm{wt}} \mathrm{BM}$ : Control $\mathrm{n}=7$; $\mathrm{AAI} n=8$; $\mathrm{AAI}+\mathrm{Dex} \mathrm{n}=11$; $\mathrm{GR}^{\mathrm{dim}}$ recipient with $\mathrm{GR}^{\mathrm{dim}} \mathrm{BM}$ : Control $n=3$; AAI $n=3$; AAI+Dex $n=3$ ) 


\subsubsection{Gene expression analysis in lung tissue of bone marrow chimeras}

The hitherto made experiments with the chimeric mice suggested a crucial role of the lung tissue rather than the BAL cells for GC-therapy of asthma. Therefore gene expression was studied by RT-qPCR in lung tissue.
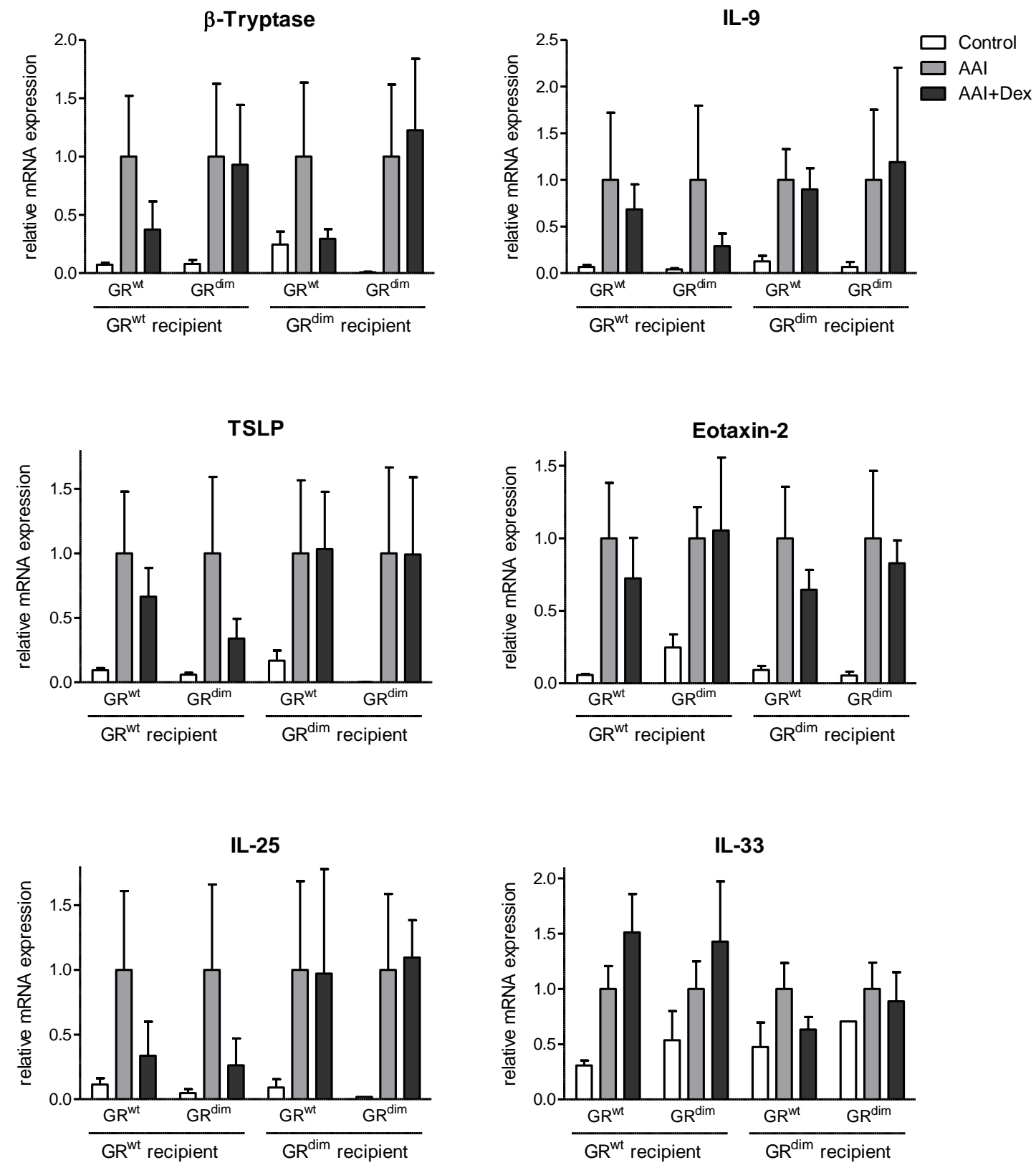

Figure 55: RT-qPCR analysis of $\beta$-Tryptase, IL-9, TSLP, Eotaxin2, IL-25 and IL-33 mRNA expression in lung tissue samples of $\mathbf{G R}^{\mathrm{wt}}$ and $\mathbf{G R}^{\mathrm{dim}}$ chimeras. The AAI induction and Dextreatment was done as described before in Figure 38. The data represent the mean mRNA expression normalized to HPRT expression. The resulting mean of the AAI sample expression was set equal to 1 . The data represent the results from 4 separate experiments $+/-\mathrm{SEM}\left(\mathrm{GR}^{\mathrm{wt}}\right.$ recipient with $\mathrm{GR}^{\mathrm{wt}} \mathrm{BM}$ : Control $n=6$; AAI $n=7$; AAI+Dex $n=8$; $\mathrm{GR}^{\mathrm{wt}}$ recipient with $\mathrm{GR}^{\mathrm{dim}} \mathrm{BM}$ : Control $\mathrm{n}=5$; AAI $\mathrm{n}=8$; $\mathrm{AAI}+$ Dex $\mathrm{n}=8$; $\mathrm{GR}^{\mathrm{dim}}$ recipient: with $\mathrm{GR}^{\mathrm{wt}} \mathrm{BM}$ : Control $\mathrm{n}=7$; AAI $\mathrm{n}=8$; AAI+Dex $\mathrm{n}=11$; $\mathrm{GR}^{\mathrm{dim}}$ recipient with GR $^{\text {dim }}$ BM: Control $n=3$; AAI $n=3$; AAI+Dex $n=3$ ) 
To investigate the effects of GC-therapy on mast cells and their activation the expression of $\beta$-Tryptase was analysed. The results of this analysis demonstrated that $\beta$-Tryptase suppression by GCs depended on the genotype of the immune system. Only chimeras with a $\mathrm{GR}^{\mathrm{wt}}$ hematopoietic system showed down-regulation of this gene after Dexamethasonetherapy.

In contrast, the expression of the $\mathrm{T}_{\mathrm{H}} 2$ cytokine IL-9 was only dependent on the genotype of the recipient mice. The lung tissue samples of $\mathrm{GR}^{\mathrm{wt}}$ mice showed a reduced expression of this cytokine in GC-treated animals. The reconstituted $\mathrm{GR}^{\mathrm{dim}}$ recipients showed no downregulation of IL-9 mRNA expression. IL-9 plays an important role in mast cell development and activation as well as in asthma pathogenesis. IL-9 also plays a role in airway remodeling and immune-cell recruitment into the lungs by promoting inflammatory mediator release such as Eotaxin. Eotaxin, as described before, plays a key role in eosinophilia development in the asthmatic lungs. The upregulation of Eotaxin2-expression during AAI could be slightly downregulated in lung samples of chimeras reconstituted with $\mathrm{GR}^{\mathrm{wt}}$ but not $\mathrm{GR}^{\mathrm{dim}}$ bone marrow.

TSLP belongs to the key mediators of asthmatic reaction. This epithelial derived cytokine not only promotes the development and migration of basophils but is also associated with increased IL-4 and IL-13 production. Its expression could be only downregulated in lung tissue of chimeras with $\mathrm{GR}^{\mathrm{wt}}$ as recipient independent on the genotype of the reconstituted immune system. Also the expression of IL-25 was only dependent on the genotype of the lung tissue and independent of the immune system genotype. The IL-25 mRNA expression could be downregulated in lung tissue of $\mathrm{GR}^{\mathrm{wt}}$ chimeras while $\mathrm{GR}^{\mathrm{dim}}$ chimeras demonstrated a similar expression level with or without Dexamethasone treatment.

IL-33 plays an important role in release of the inflammatory mediators by $\mathrm{T}$ cells, eosinophils, mast cells and basophils. It was reported that IL-33 can be produced by epithelia and myeloide cells as an alarmin for the rapid induction of IL-13-driven immunity (Prefontaine et al., 2010). The secretion of IL-33 could be not suppressed by GC-treatment independent of the genotype. Only $\mathrm{GR}^{\mathrm{dim}}$ chimera with a $\mathrm{GR}^{\mathrm{wt}}$ hematopoietic system showed a slight reduction of IL-33 mRNA expression. Opposite to this effect of Dexamethasone-treatment the expression of IL-33 in the lung tissue of $\mathrm{GR}^{\mathrm{wt}}$ chimeras was somewhat elevated upon therapy. 
RANTES

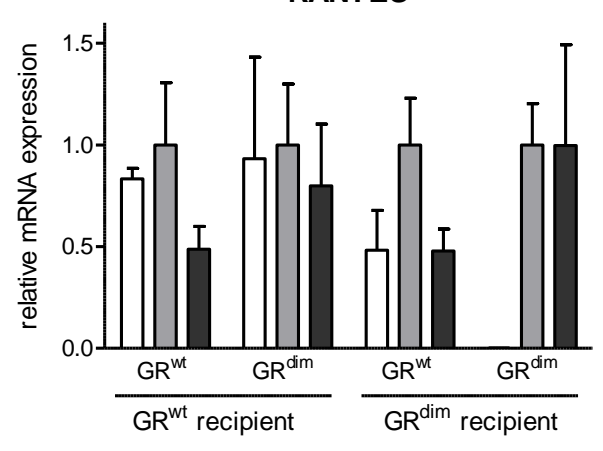

IL-4

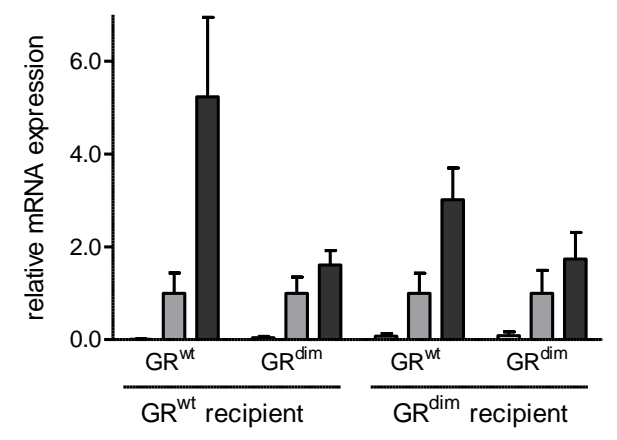

CXCR3

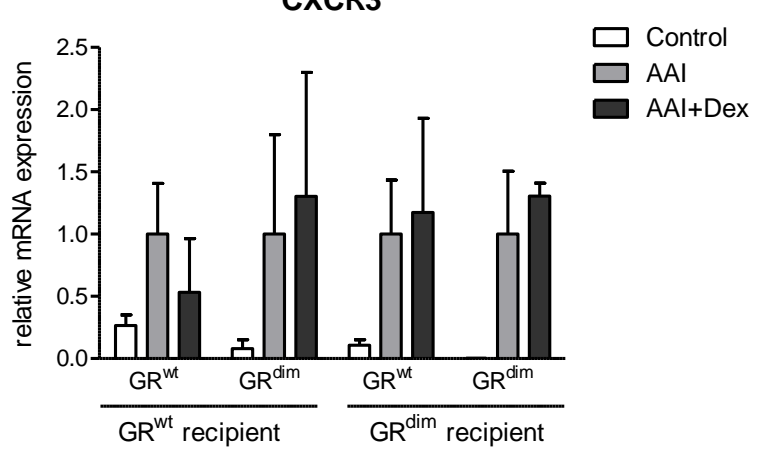

IL-13

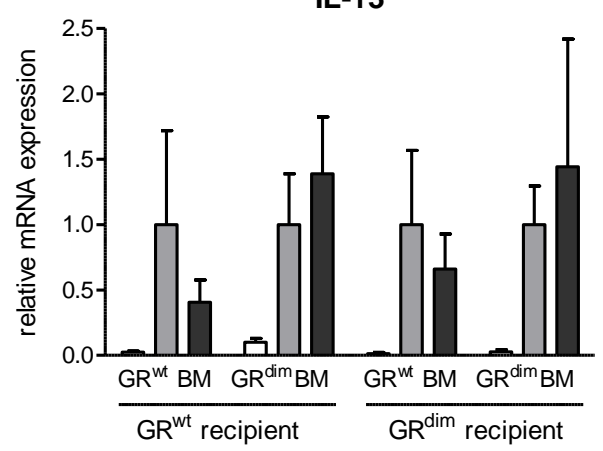

IL-5

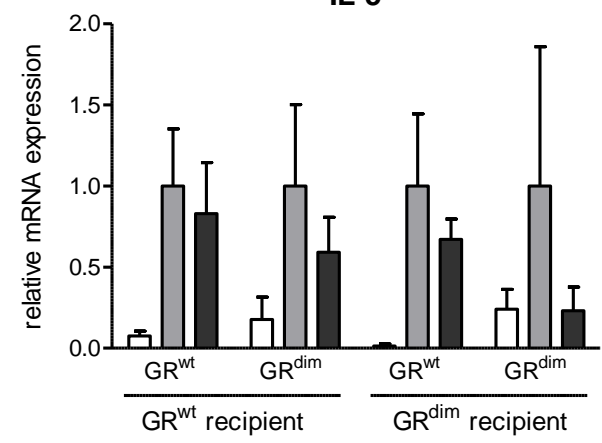

Figure 56: RT-qPCR analysis of RANTES, CXCR3, IL-4, IL-5 and IL-13 mRNA expression in lung tissue samples of $\mathbf{G R}^{\mathbf{w t}}$ and $\mathbf{G R}^{\mathrm{dim}}$ chimeras. AAI induction and Dex-treatment was done as described before in Figure 38. The data represent the mean mRNA expression normalized to HPRT expression. The resulting mean of AAI sample expression was set equal to 1 . The data represent results from 4 separate experiments $+/-$ SEM $\left(\mathrm{GR}^{\mathrm{wt}}\right.$ recipient with $\mathrm{GR}^{\mathrm{wt}} \mathrm{BM}$ : Control $\mathrm{n}=6$; AAI $n=7$; $\mathrm{AAI}+$ Dex $\mathrm{n}=8 ; \mathrm{GR}^{\mathrm{wt}}$ recipient with $\mathrm{GR}^{\mathrm{dim}} \mathrm{BM}$ : Control $\mathrm{n}=5$; $\mathrm{AAI} \mathrm{n}=8 ; \mathrm{AAI}+\mathrm{Dex} \mathrm{n}=8 ; \mathrm{GR}^{\mathrm{dim}}$ recipient with $\mathrm{GR}^{\text {wt }} \mathrm{BM}$ : Control $\mathrm{n}=7$; AAI $n=8$; AAI+Dex $n=11$; $\mathrm{GR}^{\mathrm{dim}}$ recipient with $\mathrm{GR}^{\mathrm{dim}} \mathrm{BM}$ : Control $\mathrm{n}=3$; AAI $n=3 ;$ AAI + Dex $n=3$ )

RANTES plays a critical role in recruiting of T cells, eosinophils and basophils to the site of inflammation. Although this chemokine is described to be overexpressed in asthmatic lung tissue the data from this analysis did not demonstrate a significantly induction in samples from AAI mice. The suppression of RANTES mRNA expression could be observed only in samples of the chimeras with a $\mathrm{GR}^{\mathrm{wt}}$ hematopoietic system. 
To investigate the regulation of CXCR3 mRNA expression RT-qPCR was performed. No clear effect of Dexamethasone treatment was observed due large variations. The expression of IL-4 mRNA was strongly induced in asthmatic lung tissue samples and further elevated in GC-treated animals. While the level of IL-4 mRNA expression in samples of GC-treated animals was elevated in all samples this increase of expression was stronger in chimeras with a GR ${ }^{\mathrm{wt}}$ hematopoietic system. The mRNA expression of IL-13 could be suppressed only in chimeras with a $\mathrm{GR}^{\mathrm{wt}}$ hematopoietic system independent on the genotype of the recipient.

The correlation between the IL-5 expression and the chimera type is difficult because of the strong variation of the expression data. However, it appears IL-5 mRNA expression is downregulated upon GC-therapy in all chimera types independent on the genotype of the lung cells or the reconstituted hematopoietic system. The expression of iNOS was induced in asthmatic lung tissue. The treatment of the chimeras with GCs led to a suppression of iNOS mRNA expression in all chimeras independent of their genotype.

Occludin is a protein located in the tight junctions. According to the current literature this protein plays an important role for the maintenance of epithelial barriers functions (Xiao et al., 2013). Because of the loss of proper barrier function during an asthmatic reaction, the expression of Occludin in GC-treated animals was investigated. The data from the RT-qPCR analysis of lung tissue samples demonstrated a slightly decrease in mRNA expression in all GC-treated chimeras except for $\mathrm{GR}^{\mathrm{wt}}$ chimeras with a $\mathrm{GR}^{\mathrm{dim}}$ immune system.

The expression of MCP-1 was elevated in all chimeras after the GC-therapy except for $\mathrm{GR}^{\mathrm{dim}}$ chimera reconstituted with $\mathrm{GR}^{\mathrm{dim}}$ bone marrow. IL-10 expression was induced after the development of an asthmatic reaction in lung tissue. This overexpression of IL-10 could be suppressed in $\mathrm{GR}^{\mathrm{wt}}$ chimera by GC-treatment independent of the immune system genotype. IL-10 mRNA expression in the samples of GC-treated GR ${ }^{\mathrm{dim}}$ chimeras was not suppressed.

CXCL12 (or SDF-1) is an inflammatory cytokine that is chemotactic for inflammatory cells such macrophages. This cytokine exerts its function through CXCR4. CXCR4 (or SDF-1 receptor) expression is upregulated during AAI while its expression in healthy lungs is nearly absent. The data show no strongly upregulation of CXCL12 mRNA expression after induction of an asthmatic reaction except for $\mathrm{GR}^{\mathrm{wt}}$ chimeras with a $\mathrm{GR}^{\mathrm{wt}}$ immune system while the expression of CXCR4 was elevated in all asthmatic chimeras. GC-therapy led to a strong 
suppression of CXCR4 expression in all Dexamethasone-treated chimeras independent of the genotype while the expression of CXCL12 was only slightly decreased. The expression of CXCL12 in lung samples of $\mathrm{GR}^{\mathrm{wt}}$ chimera with a $\mathrm{GR}^{\mathrm{dim}}$ hematopoietic system remained nearly unchanged in all samples independent on the treatment of the animals.
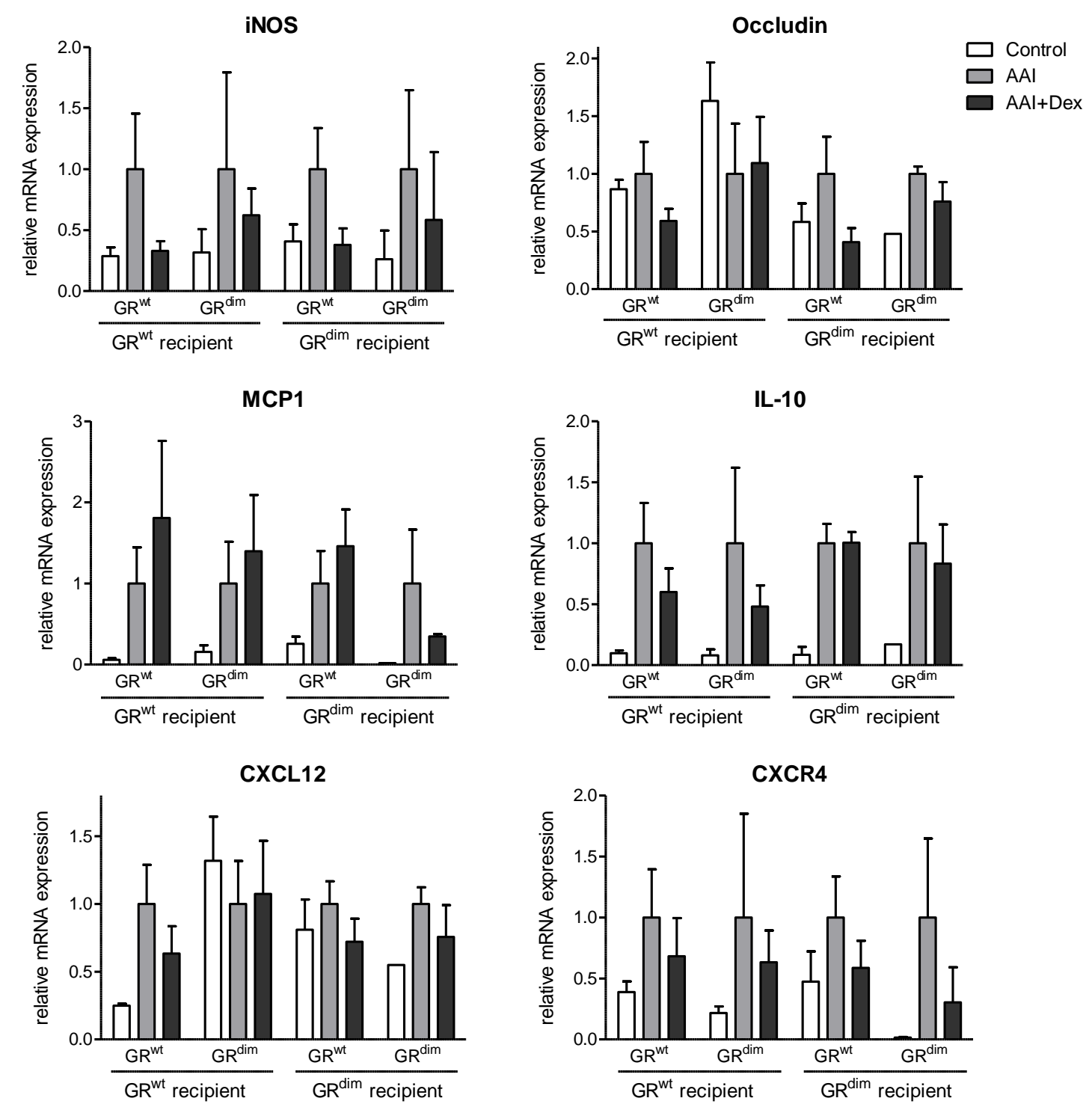

Figure 57: RT-qPCR analysis of iNOS, Occludin, MCP-1, IL-10, CXCL12 and CXCR4 mRNA expression in lung tissue samples of $\mathbf{G R}^{\mathrm{wt}}$ and $\mathbf{G R}^{\mathrm{dim}}$ chimeras. AAI induction and Dex-treatment was done as described before in Figure 38. The data represent the mean mRNA expression normalized to HPRT expression. The resulting mean of AAI sample expression was set equal to 1 . The data represent results from 4 separate experiments $+/$ - SEM $\left(\mathrm{GR}^{\mathrm{wt}}\right.$ recipient with $\mathrm{GR}^{\mathrm{wt}} \mathrm{BM}$ : Control $\mathrm{n}=6$; AAI $n=7$; AAI+Dex $n=8 ; \mathrm{GR}^{\text {wt }}$ recipient with $\mathrm{GR}^{\text {dim }}$ BM: Control $n=5$; AAI $n=8$; AAI+Dex $n=8$; $\mathrm{GR}^{\mathrm{dim}}$ recipient with $\mathrm{GR}^{\mathrm{wt}} \mathrm{BM}$ : Control $\mathrm{n}=7$; $\mathrm{AAI} \mathrm{n}=8$; $\mathrm{AAI}+\mathrm{Dex} \mathrm{n}=11 ; \mathrm{GR}^{\mathrm{dim}}$ recipient with $\mathrm{GR}^{\mathrm{dim}}$ BM: Control n=3; AAI n=3; AAI+Dex n=3) 


\subsubsection{Analysis of $\mathbf{G R}^{\mathrm{SPC} \text {.reERT2 }}$ mice}

In recent years it turned out that non-immune cells of the lungs play an important role during the development of allergic reactions. While it is clear that these cells are involved in asthma pathogeneses their relevance for the treatment of asthma is yet unclear.

To analyze the role of epithelial cells of the lungs for GC-therapy of AAI inducible AT2spezific GR-knockout mice $\left(\mathrm{GR}^{\mathrm{SPC} \text { SreERT2}}\right.$ ) were used. To induce the ablation of the GR in

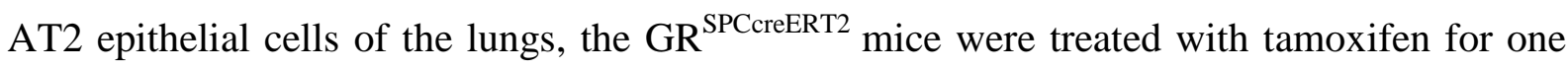
week. To investigate the efficiency of the tamoxifen-inducible GR-knockout system the AT2 cells were isolated from lung tissue of wild type and $\mathrm{GR}^{\mathrm{SPC} \text { (creER2 }}$ mice by FACS sorting. After isolation of RNA from separated AT2 cells GR gene expression was analyzed by RTqPCR. The relative GR mRNA expression data show that the tamoxifen treatment of $\mathrm{GR}^{\mathrm{SPC} \text { creERT2 }}$ mice successfully reduced GR expression in AT2 cells. The remaining expression of the GR in $\mathrm{GR}^{\text {SPCcreERT2 }}$ cells was less than $35 \%$ of GR-expression in $\mathrm{GR}^{\text {flox }}$ mice (Figure 58).

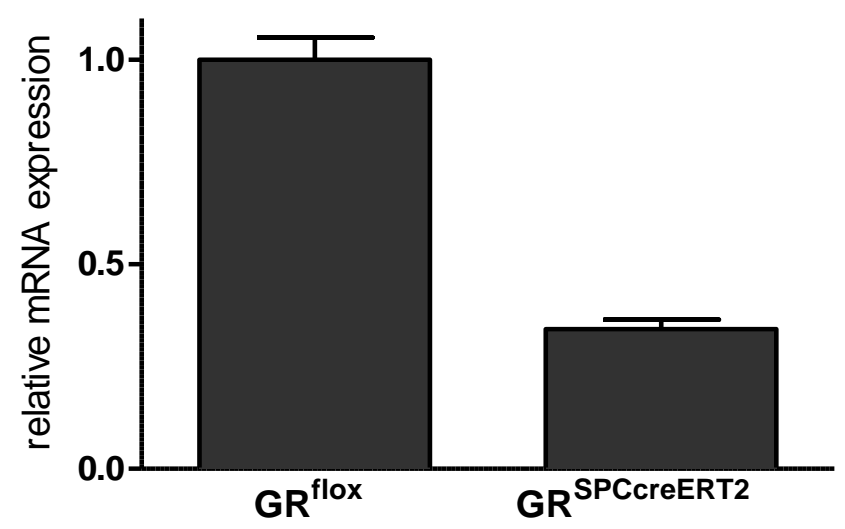

Figure 58: Analysis of GR mRNA expression in AT2 cells of $\mathbf{G R}^{\text {flox }}$ and $\mathbf{G R}^{\text {SPCcreERT2 }}$ mice. To induce the GR knockout in AT2 cells GR ${ }^{\text {SPCreERT2 }}$ mice were treated with tamoxifen for one week. 2 days after the last tamoxifen administration the mice were used for induction of AAI as described before in Figure 38. The FACS sorted AT2 cells from $\mathrm{GR}^{\text {flox }}$ and $\mathrm{GR}^{\text {SPCcreER2 }}$ mice were used for gene expression analysis by RT-qPCR. The RT-qPCR was repeated twice. The data show the mean relative GR mRNA expression +/-SEM. The expression data was normalized to HPRT expression and the mean expression of $\mathrm{GR}^{\text {flox }}$ samples was set equal to 1 . $\left(\mathrm{GR}^{\text {flox }} \mathrm{n}=2 ; \mathrm{GR}^{\mathrm{SPC} \text { creERT2 }} \mathrm{n}=7\right)$

GR mRNA expression in AT2 epithelial cells of $\mathrm{GR}^{\mathrm{SPC} C r e E R T 2}$ mice is significantly $(p<0.0001)$ reduced. Hence, this mutant strain is a suitable tool to understand AT2 cell function in lung disease treatment with GCs. 


\subsubsection{BAL analysis}

The induction of an asthma like reaction in $\mathrm{GR}^{\mathrm{SPC} C r e \mathrm{RT} 2}$ mice led to a significantly increased number of infiltrating cells in the BAL. The ablation of the GR in AT2 cells did not impair this inflammatory response (Figure 59). GC-therapy of these mutant mice did not suppress this cellular lung infiltration in the same way as in wild type mice. While the BAL of GCtreated wild type animals contained less than 150000 cells the BAL of GR $^{\text {SPCcreER2 }}$ mice have more than 700000 infiltrating cells. This means about $90 \%$ reduction of infiltrating cells in wild type mice and only $40 \%$ reduction in BAL samples of GC-treated GR ${ }^{\text {SPCcreERT2 }}$ mice.

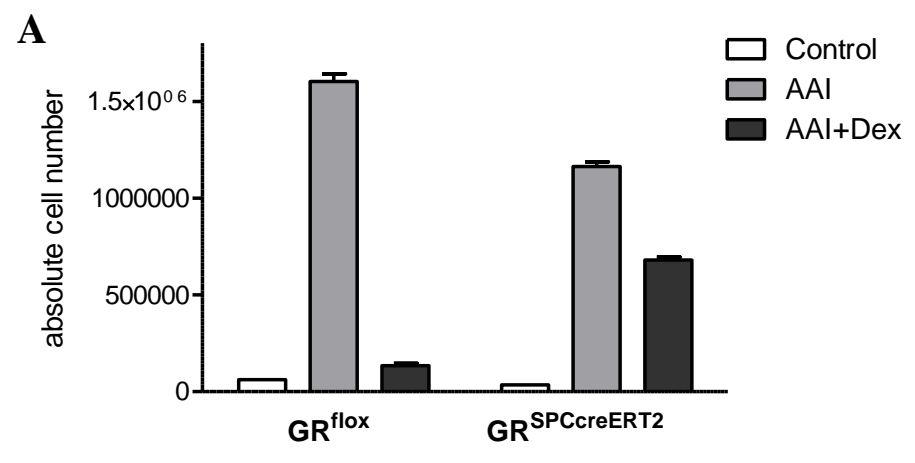

B

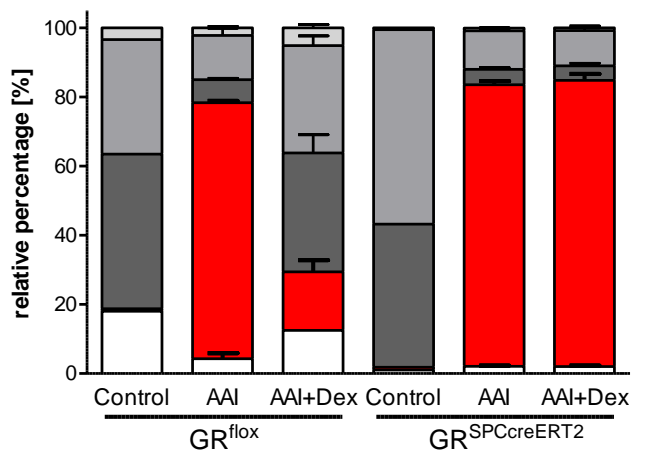

C

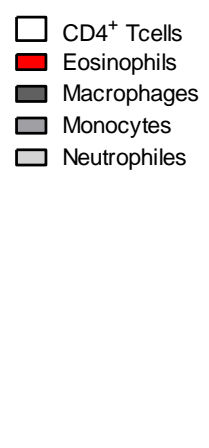

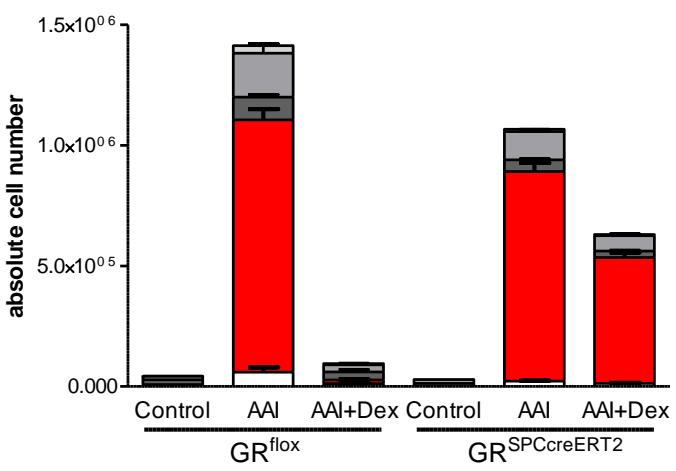

Figure 59: BAL cell composition and relative percentages of BAL subpopulations of GR $^{\text {flox }}$ and $\mathbf{G R}^{\text {SPCreERT2 }}$ mice. The BAL of healthy (control), AAI and Dexamethasone-treated (AAI+Dex) mice was quantified for absolute cell number with the help of a Neubauer cell count chamber. To investigate the BAL cell subpopulations the isolated BAL samples were analyzed by FACS. The relative percentages and absolute cell number of the BAL were used for the calculation of the absolute cell numbers of the subpopulations. The data represent the mean absolute cell number and the relative percentage +/- SEM (GR ${ }^{\text {flox }}$ : control $n=1$; AAI $n=2 ;$ AAI+Dex $n=2 ;$ GR $^{\text {SPCcreERT2}}$; control $n=1$; AAI $\mathrm{n}=3$; AAI + Dex $n=3$ )

The cell composition of the BAL from control and asthmatic mice showed no differences between wild type and $\mathrm{GR}^{\mathrm{SPC} \text { PreERT2 }}$. The cellular influx in allergic lungs was dominated by eosinophilic granulocytes and their relative percentage was about $80 \%$ of all BAL cells in 
both mouse strains. The BAL of control mice consisted of macrophages and monocytes and showed no sign of inflammation or infection (hardly any neutrophils). However, the response to GC-therapy revealed significant differences dependent on GR expression in AT2 cells. While the asthmatic mice with normal GR expression level showed strongly reduced relative percentage and cell number of eosinophils (Figure 59) GC-therapy of GR ${ }^{\text {SPCreERT2 }}$ mice did not affect the eosinophilia in the lungs at all.

\subsubsection{Ova-specific immunoglobulin production in $\mathbf{G R}^{\mathrm{SPC} C r e E R T 2}$ mice}

In order to investigate the Ig class switching after induction of an asthma-like reaction in $\mathrm{GR}^{\mathrm{SPC} C r e E R T 2}$ mice after tamoxifen treatment the serum samples were analyzed for Ovaspecific IgE, IgG2a and IgG1. Immunization with Ova induced significantly increased production of Igs. The induction did not show any differences between wild type and $\mathrm{GR}^{\text {SPCcreERT2 }}$ animals.

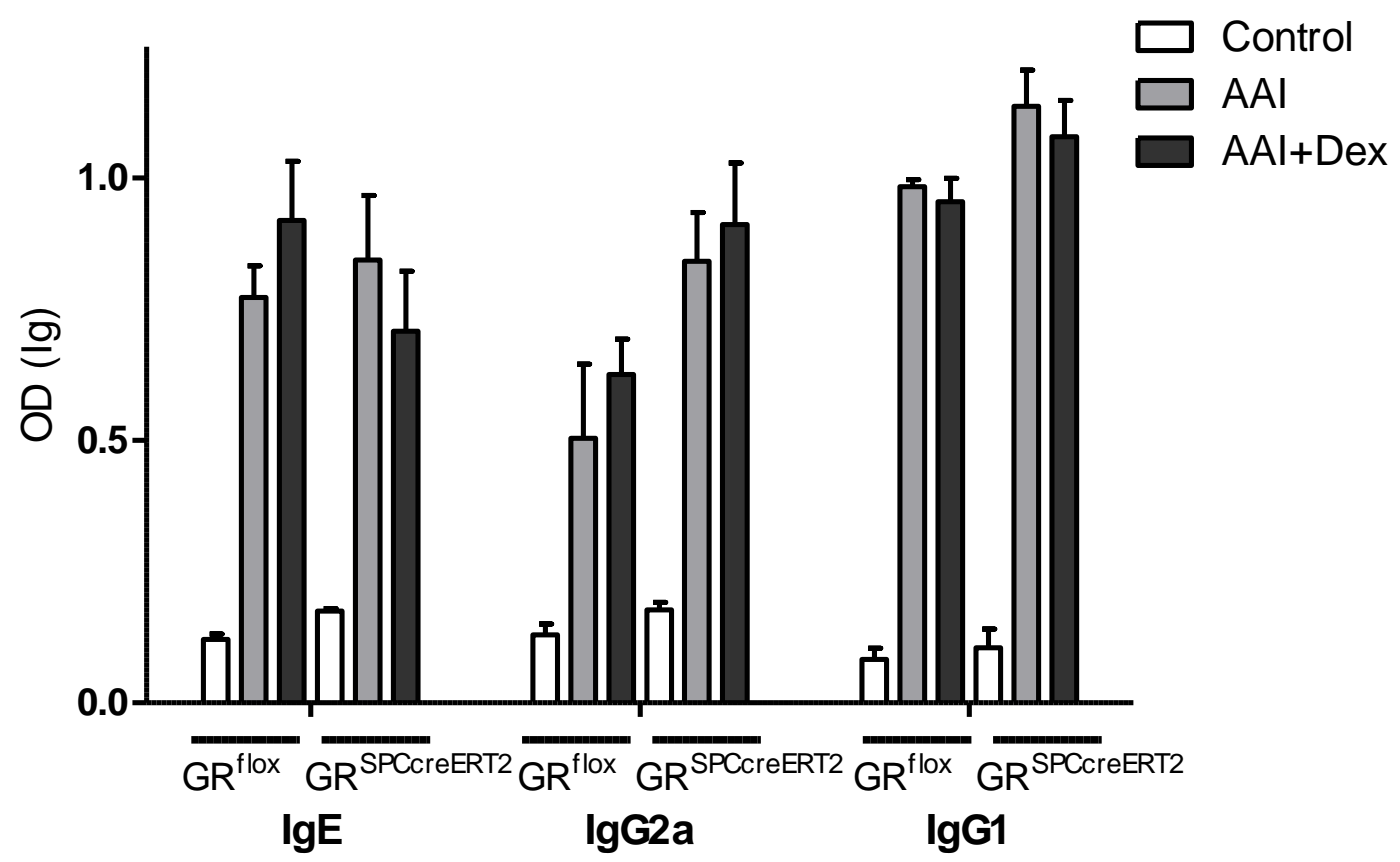

Figure 60: Analysis of immunoglobulin production in serum of $\mathbf{G R}^{\text {flox }}$ and $\mathbf{G R}^{\text {SPCcreER2 }}$ mice. The induction of an asthma-like reaction led to a significantly increased production of Ova-specific IgE, IgG2a and IgG1 in $\mathrm{GR}^{\text {flox }}$ and $\mathrm{GR}^{\text {SPCreERT2 }}$ mice. The data show the mean OD +/- SEM $\left(\mathrm{GR}^{\text {flox }}\right.$ : control $n=1 ;$ AAI $n=2 ;$ AAI+Dex n=2; GR $^{\text {SPCcreRT2 }}$; control $n=1 ;$ AAI n=3; AAI+Dex n=3) 


\subsubsection{Gene expression analysis in AT2 cells from $\mathrm{GR}^{\mathrm{SPC} C r e E R T 2}$ mice}

It is currently believed that the airway epithelium is a major source of cytokines, chemokines and other inflammatory mediators during asthmatic reactions in the lungs. Therefore, in addition to its respiratory and barrier function, it plays an important role for the immune response, the modulation of inflammatory diseases and providing local host defense. The airway epithelium contains of several cell types which have different functions.

From an immunological point of view AT2 cells are most interesting. They play the role of professional APCs and can present antigens on MHCII molecules. Additionally, AT2 cells can produce a large spectrum of inflammatory mediators that modulate inflammation and affect the adoptive and innate immune response in the lungs.

Since the lung epithelium is a major source of diverse chemokines, cytokines and other inflammatory mediators it was interesting to investigate the modulation of gene expression by GC-therapy. To this end the AT2 cells were isolated by FACS sorting and the modulation was analyzed by RT-qPCR.

The results of RT-qPCR approach demonstrate that the induction of an asthma-like reaction in the lungs of $\mathrm{GR}^{\text {flox }}$ and $\mathrm{GR}^{\text {SPCcreERT2 }}$ mice leads to an increased expression of a large spectrum of inflammatory mediators. The mRNA expression of important cytokines and chemokines such IL-1 $\beta$, IL-33, IL-6, IL-4, IL-13, IL-25 and Eotaxin-2 was strongly increased in the samples of animals with an asthma-like reaction. The treatment with GCs led to a strong suppression of IL-1 $\beta$ mRNA expression in GR $^{\text {flox }}$ mice while the expression in GR ${ }^{\text {SPCcreERT2 }}$ AT2 cells was slightly increased. The same response to the treatment could be observed in the analysis of IL-33, IL-6, Eotaxin-2, RANTES, iNOS and TNF- $\alpha$. The expression of these genes could be downregulated by Dexamethasone only in samples of GR $^{\text {flox }}$ AT2 cells (Figure 61). 

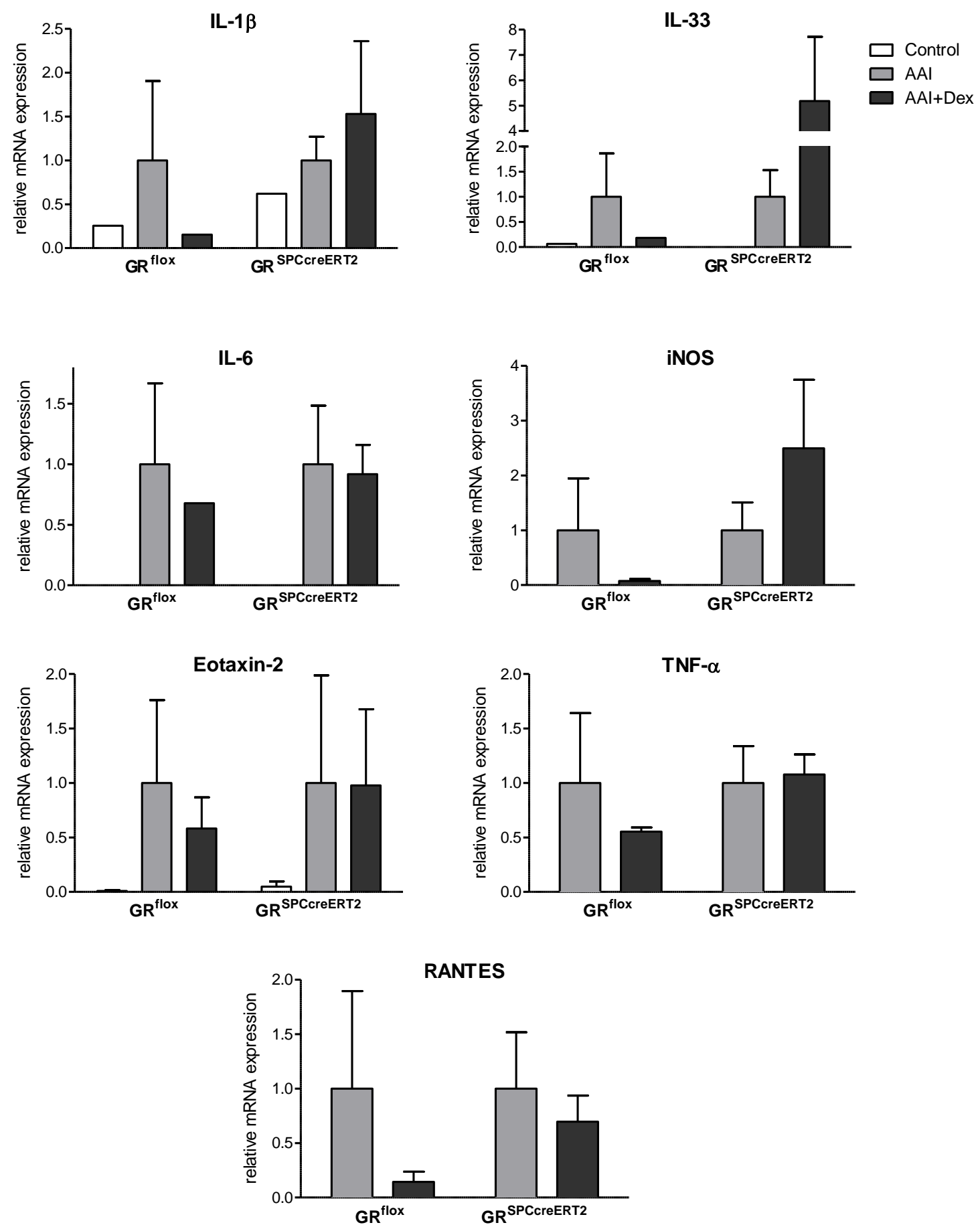

Figure 61: Gene expression analysis of inflammatory mediators IL-1ß, IL-33, IL-6, iNOS, Eotaxin2, TNF- $\alpha$ and RANTES in AT2 cells from $\mathbf{G R}^{\text {flox }}$ and $\mathbf{G R}^{\text {SPCreERT2 }}$ mice. AT2 cells were isolated from the lungs of healthy, AAI and Dex-treated AAI mice. The asthma-like reaction was induced like described before in Figure 10.The data represent the normalized (on the AAI-samples) mean of the relative mRNA expression $+/$ - SEM $\left(\mathrm{GR}^{\text {flox }}\right.$ : control $n=1$; AAI $n=2$; AAI+Dex $n=2$; $\mathrm{GR}^{\mathrm{SPC} C r e E R T 2}$ : control $\mathrm{n}=1$; AAI $\mathrm{n}=3$; AAI+Dex $\mathrm{n}=3$ ) 
At the same time, the expression of TSLP, ZO-1 and Occludin were increased after GCtherapy in AT2 cells of both genotypes but to a different extent. Interestingly the enhancement of TSLP expression after Dex-therapy was stronger in AT2 samples from GR ${ }^{\text {SPCreERT2 }}$. While the ZO-1 expression in Dex-treated AT2 cells from wild type animals was about three-fold higher than the expression in untreated mice with AAI, this effect was nearly absent in $\mathrm{GR}^{\mathrm{SPC} \text { creERT2 }}$ samples.
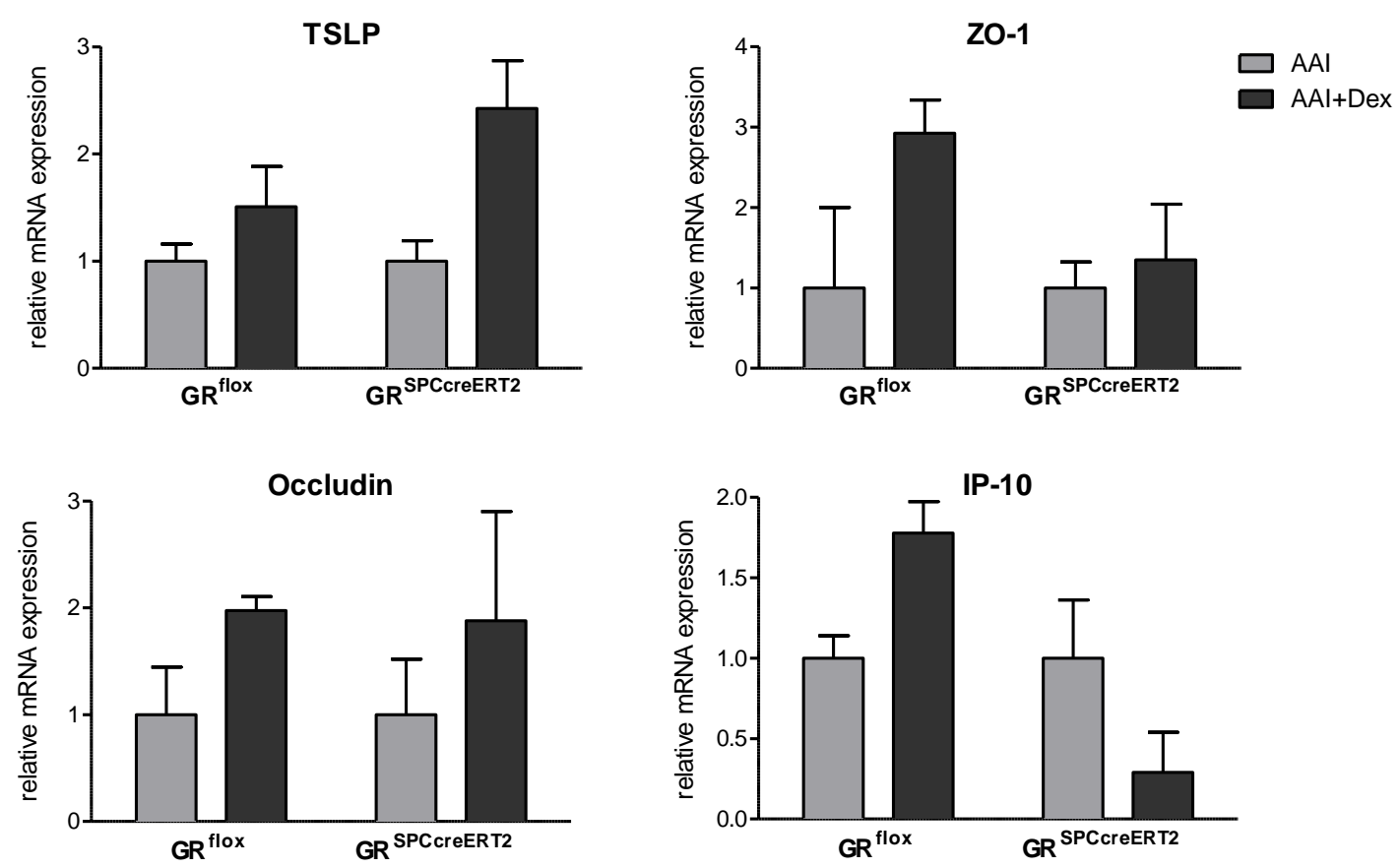

Figure 62: Gene expression analysis of TSLP, ZO-1, Occludin and IP-10 of isolated AT2 cells from GR $\mathbf{G R}^{\text {flox }}$ and $\mathbf{G R}^{\text {SPCreERT2 }}$ mice. AT2 cells were isolated from lung samples of healthy, AAI and Dex-treated AAI mice. The asthma-like reaction was induced like described before in Figure 10.The analysis data represent the normalized (on the AAI-samples) mean of the relative mRNA expression +/- SEM $\left(\right.$ GR $^{\text {flox }}$ : control $n=1 ;$ AAI $n=2 ;$ AAI+Dex $n=2 ;$ GR $^{\text {SPCcreERT2}}$; control $n=1 ;$ AAI $n=3$; AAI+Dex $n=3$ )

While the induction of Occludin expression by GCs was comparable between GR $^{\text {flox }}$ and $\mathrm{GR}^{\text {SPCcreERT2 }}$ AT2 cells, the IP-10 expression demonstrated large differences concerning the GC-effects. GC-therapy led to an increased IP-10 expression in GR ${ }^{\text {flox }}$ AT2 cells while IP-10 was reduced in samples of $\mathrm{GR}^{\mathrm{SPC} \text { (creERT2 }}$ cells (Figure 62). 


\section{Discussion}

\subsection{Asthma and the experimental mouse model of allergic airway inflammation}

Allergic asthma is an inflammatory disease with constantly rising incidence in the industrialized countries. According to the World Health Organization, more than 235 million people currently suffer from this respiratory disorder. Because the cause and risk factors for asthma development are only poorly understood, a plethora of studies have been performed investigating this topic. Beside genome-wide linkage screens of asthmatic and healthy people, experimental mouse models offer the opportunity to study the pathogenesis and development of asthma. Although mouse models can not completely reproduce the pathophysiology of human asthma mice develop a strong allergic airway inflammation, after sensitization and challenge with antigens, with a bright spectrum of characteristic clinical symptoms including eosinophilia, airway hyperresponsiveness, mucus hypersecretion and increased IgE level. The asthma-like reaction that was used in this work is achieved by immunizing and challenging the mice with Ovalbumin as the antigen in combination with Alum as a potent adjuvant. The repeated immunizations and challenges induce a robust, allergic pulmonary inflammation in laboratory rodents (Kumar et al., 2008).

The AAI model that was used in this work included a strong cellular infiltration of the lungs with immune cells. This effect was seen by analyzing BAL samples by FACS and lung sections by histology, respectively. The wild type and the diverse mutant mice developed comparable lung infiltration after induction of AAI independent of the background. Opposite to several other studies, C57BL/6 mice developed nearly the same level of inflammation after induction of AAI. While the cell number in the BAL of healthy mice was dominated by macrophages and consisted of less than $1 \times 10^{5}$ cells, induction of AAI increased lung infiltration to up to $2 \times 10^{6}$ cells dominated by eosinophils (60-80\%). The absolute cell numbers of lymphocytes, macrophages and neutrophiles were also increased in the BAL of mice with AAI. The relative percentage of the different cell populations in the BAL revealed that although the absolute cell numbers were increased after AAI induction the percentages of macrophages and neutrophiles were lower in diseased mice compared to healthy animals. The data from the FACS analysis of the BAL correlated with the histological studies. While the healthy lung showed a "spongy" structure with thin-walled alveoli and free airflow conducting airways the lung sections of mice with AAI demonstrated a dramatic lung inflammation with cellular and mucosal infiltration. The inflammation was largely located in 
the peribronchial and perivascular areas but could also be seen in the lung parenchyma. The characteristic airway remodeling seen in severe asthma in humans occurs only in a mild form in the used mouse model because of the short inflammation phase. Nonetheless, first signs of airway deformation could already be observed: mucus hypersecretion led to a hyperplasia of the goblet cells and the asthmatic airways were characterized by a "waved" airway wall with reduced free space for gas exchange.

Beside infiltration and mucus hypersecretion, the mice with AAI demonstrated strongly elevated levels of antigen-specific immunoglobulin production. The sensitization to Ovalbumin successfully led to an isotype switch to $\operatorname{IgE}$, IgG1 and IgG2a in both mouse strains. Opposite to several publications the C57BL/6 mice with AAI produced only slightly reduced amount of immunoglobulins compared to the BALB/c mouse strain. Based on these observations the AAI induction protocol could be successfully used for eliciting an asthmalike reaction in the subsequent experiments with different mouse strains on a BALB/c or a C57BL/6 background without changes in the protocol.

\subsubsection{Establishment of the GC therapy}

The aim of this work was to investigate the mechanisms of GCs in the treatment of asthmatic reactions. After the establishment of the AAI model, the treatability of wild type mice was analyzed. The data from several experiments with BALB/c and C57BL/6 mice demonstrated, that potent effects of GCs on airway inflammation could be achieved already after two intraperitoneal injections with Dexamethasone (10 mg per $1 \mathrm{~kg}$ body weight). GC therapy led to a strong reduction of the number of BAL cells in both mouse strains. This effect was mainly based on the significant suppression of lung eosinophilia. The BAL isolated from the animals with AAI contained more than $1 \times 10^{6}$ eosinophils (about $70 \%$ of all BAL cells) while the eosinophils in GC-treated mice made up less than $40 \%$ of the whole BAL. In contrast, GC therapy did not show any effects on $\operatorname{IgE}, \operatorname{IgG} 2 \mathrm{a}$ and $\mathrm{IgG} 1$ production independent of the genetic background. Although the production of these immunoglobulin isotypes plays an important role in the pathogenesis of asthma the reduction of their concentration in serum does not seems to be critical for the therapeutic efficiency of GCs. Possible explanations for the absent reduction of immunoglobulin level after GC therapy could be the mechanism published by Jabara and Zieg. They reported that high dose therapy with GCs may maintain high immunoglobulin levels via upregulation of CD40 ligand expression and induction of CD40L-dependent immunoglobulin isotype switching (Jabara et 
al., 2001; Zieg et al., 1994). Another possible reason for the high levels of IgE, IgG2a and IgG1 in the serum irrespective of GC treatment could be the short duration of the therapeutic phase.

\subsection{Role of GR DNA-binding and dimerization for GC-therapy of AAI as a model of Asthma}

GCs were introduced into the clinics as a powerful therapy for many inflammatory diseases more than half a century ago (Lühder and Reichardt, 2009). Although these drugs are amongst the most widely prescribed anti-allergic compounds worldwide the precise mechanism of GC action is not fully understood. The current knowledge assumes that the efficiency of GC therapy dependents on a variety of mechanisms addicted to different disease type (Baschant et al., 2012). Based on these findings it seems to be impossible to define a single mode of GC action or target cell type for all inflammatory diseases. As described before, the majority of GC effects are mediated by the GR via transcriptional activation or repression of target genes. The first mode of action requires dimerization of the GR, its binding to regulatory DNA elements and subsequent activation of target genes. Alternatively, the GR may control transcription via transrepression in a DNA binding-independent manner, which is mediated by tethering interactions with other transcription factors. The potent immunosuppressive effects of GC therapy were reported to be largely dependent on the inhibition of pro-inflammatory gene expression via transrepression while the transactivation was rather thought to account for many side effects.

To determine the requirement of GR DNA-binding for asthma, the efficiency of GCs in the treatment of AAI was compared between wild type and $\mathrm{GR}^{\mathrm{dim}}$ mice. The goal of this study was to evaluate the relevance of the two major modes of GC action. Based on the observations from the histological sections, NIR Imaging and the FACS data both mouse strains developed a comparably strong inflammation in the lungs after AAI induction. The infiltration see in histological sections was mostly located close to the wall of the conducting airways and blood vessels but was also present in the alveolar tissue. Additionally, the airway epithelium showed the first remodelling stages with hyperplasia of goblet cells and mucus hypersecretion. The cellular infiltration in both strains consisted of about $70 \%$ eosinophils after AAI induction. In contrast, the main population in the airways of healthy mice was identified as macrophages. This replacement in cell populations was seen in both genotypes and is characteristic for asthmatic reactions. Importantly, the anti-inflammatory effect of GC 
therapy on lung infiltration could only be seen in treated wild type mice whereas the inflammation in the $\mathrm{GR}^{\mathrm{dim}}$ mice remained almost unchanged. While the cell number of eosinophils in wild type mice was significantly reduced from more than $1 \times 10^{6}$ cells in AAI to about $1 \times 10^{5}$ eosinophils in Dex-treated mice, the treatment of $\mathrm{GR}^{\mathrm{dim}}$ led only to a minimal reduction. After GC-therapy the BAL of $\mathrm{GR}^{\mathrm{dim}}$ mice still consisted of more than $60 \%$ of eosinophils and the change in eosinophil numbers after GC-therapy was not significant. The same effects of GC therapy could be seen in lungs sections. While the GC treatment of wild type mice led to a strongly reduced mucus secretion and cellular infiltration accompanied by an enlargement of the free space for gas exchange, these anti-inflammatory effects were absent in Dex-treated GR ${ }^{\text {dim }}$ mice. The treatment of wild type animals with GCs did not only lead to the reduction of cellular infliltration and eosinophilia but also to the replenishment of macrophages in the lungs. Because macrophages play an important role for the treatment of allergic reactions and are necessary for the healing process after the inflammation of tissues this recurrence can be seen as sign of successfully therapy. The population of neutrophiles was not reduced by GC therapy in both mouse strains. Opposite to the effect on eosinophils, the relative percentage of neutrophils was even increased after Dex-therapy. This ambivalent effect of GCs on different granulocyte populations was already observed previously in several other studies. A current report suggests that the persistent airway neutrophilia observed in patients with asthma might be caused by the failure of GCs to suppress IL- 8 mRNA expression (Fukakusa et al., 2005). The neutrophilia was also reported to correlate with asthma severity (Wenzel et al., 1997). On the one hand these granulocytes play a role in the initiation and resolution of an asthma attack but on the other hand these cells are also important for the digestion of airway mucus plugs, which block the airways during acute asthma (Innes et al., 2008). Because of this controversial role it is difficult to speculate on the role of GC effects on neutrophils.

To further investigate the role of the DNA-binding dependent mode of the GR, proliferation and priming of antigen-specific $\mathrm{T}$ and $\mathrm{B}$ cells were analysed ex vivo. Ova-restimulated splenocytes from AAI mice showed increased proliferation and an elevated production of IL-2, IL-4 and IFNy. The data from the ${ }^{3} \mathrm{H}$-thymidine incorporation assay revealed no differences in proliferation between AAI and GC-treated mice of both genotypes. However, the analysis of the IL-2 concentration in the supernatants of these splenocytes demonstrated not only a strong increase in the IL-2 secretion in AAI samples but also a significantly reduction of its production in samples from GC treated wild type mice. This suppression of 
IL-2 production by GC therapy was absent in $\mathrm{GR}^{\mathrm{dim}}$ samples. One possibility to explain this observation could be different effects of GCs on B and T cells. It is possible that although proliferation and activation of $\mathrm{T}$ cells can be successfully reduced by GC therapy, $\mathrm{B}$ cell proliferation remained unaltered. The data on IL-4 and IFNy secretion support this hypothesis. IFNy production was strongly increased in samples from wild type and $\mathrm{GR}^{\mathrm{dim}}$ mice with AAI in comparison to control splenocytes from healthy mice. Nonetheless, GCtherapy led to a reduced IFNy secretion only in samples from wild type mice. Opposite to this effect, the increased production of IL-4 in samples from wild type and GR ${ }^{\mathrm{dim}}$ mice with AAI could not be suppressed by GC-treatment in any strain. This effect on IL-4 could be explained by the influence of GCs on the $\mathrm{T}_{\mathrm{H}} 1 / \mathrm{T}_{\mathrm{H}} 2$ balance. According to several reports GCs may cause a selective suppression of $\mathrm{T}_{\mathrm{H}} 1$ immunity and a shift toward $\mathrm{T}_{\mathrm{H}} 2$-mediated humoral immunity. GCs may inhibit the production of $\mathrm{T}_{\mathrm{H}} 1$ cytokines including IFNy by APCs and $\mathrm{T}_{\mathrm{H}} 1$ cells, but upregulate the production of $\mathrm{T}_{\mathrm{H}} 2$ cytokines, like IL-4, by $\mathrm{T}_{\mathrm{H}} 2$ cells (Elenkov., 2004). Another explanation comes from the fact that IL-4 unlike IFNy can be produced not only by $\mathrm{T}$ cells but also by B cells. Because of this, the observed dissimilarity of cytokine regulation by GCs could depend on the different effects of GCs on B and T cells. While the suppression of T cell activation and proliferation seems to be dependent on DNA-binding mode of GR action the effects on B cells did not show any observable correlation with the absence or presence of this mode of GR action.

To investigate the requirement of GR DNA-binding on the secretion of other proinflammatory cytokines the IL-5 concentration was analysed in serum samples from wild type and GR ${ }^{\text {dim }}$ mice. Because IL-5 plays a critical role in the maturation and release of eosinophils from the bone marrow the increased secretion of this cytokine plays an important role in the pathogenesis of asthma. This cytokine can be produced by $\mathrm{T}_{\mathrm{H}} 2$ cells but also by mast cells and eosinophils and act in an autocrine manner. An increased IL-5 concentration could be seen in serum samples of AAI mice of both genotypes but GC-therapy did not lead to a major change in both genotypes.

Additionally, the serum of wild type and GR ${ }^{\text {dim }}$ animals was analyzed for antigen-specific immunoglobulin levels. The induction of AAI led to a strong increase of IgE, IgG2a and IgG1 production in mice of both genotypes. The serum samples of GC-treated animals showed no differences in immunoglobulin secretion irrespective of the genotype. 


\subsubsection{Effects of GCs on the barrier function of the lung endothelium}

The altered microvascular permeability was proposed to be one of the critical factors of asthma pathogenesis. The increased permeability and the loss of proper barrier function, which occur in asthmatic lungs, lead to different pathogenic effects including the generation of airway submucosal and mucosal oedema consisting of plasma exudates (Persson., 1986) and an increased change for a contact of APCs with antigen and the infiltration of the lungs with immune cells. It is currently believed that this increased permeability can cause an increased responsiveness of the airways in asthma. In this work the barrier function of the lung endothelium was analysed using the Evans blue extravasation technique. The data from this experiment demonstrated a strong correlation between Evans blue dye leakage and airway inflammation. The concentration of Evans blue in the lung tissue of AAI mice was significantly increased compared to samples from healthy animals. While the increased permeability could be successfully suppressed by GC-therapy in the lungs of wild type mice the barrier function of $\mathrm{GR}^{\mathrm{dim}}$ mice could be not improved by Dexamethasone treatment. Based on this observation, it is possible that the DNA-binding dependent GR action is necessary for the recovery of the endothelial barrier function during therapy. This therapeutic effect could be based on many factors including the effects on the cytoskeleton and tight junction proteins. Several publications reported that in this context the tight junction transmembrane proteins occludin and ZO-1 play a crucial role for microvascular permeability (Hirase et al., 1997; Xiao et al., 2013). This hypothesis was tested later using $\mathrm{GR}^{\mathrm{dim}}$ bone marrow chimeras and $\mathrm{GR}^{\mathrm{SPC} \text { SreERT2 }}$ mice (see below).

\subsubsection{Effects of GCs on gene expression in BAL and lung parenchymal cells}

The effects of GC therapy on inflammation are thought to strongly depend on the regulation of gene expression. The GR is known to modulate the expression of many genes either by transactivation or transrepression. In order to investigate differences in gene regulation in the absence of the DNA-binding dependent mode of GR action, samples from wild type and $\mathrm{GR}^{\mathrm{dim}}$ mice were analyzed via RT-qPCR. Since a variety of cytokines, chemokines and other inflammatory mediators are involved in asthmatic reactions but may be differently regulated dependent on the cell type, the analysis was done with BAL samples and lung samples separately. These independent analyses of gene expression provided the opportunity to investigate the influence of GCs on the infiltrating cells as well as inflamed tissue. The 
allergic response in the lungs led to an increased expression of many pro-inflammatory genes in BAL and lung tissue samples.

The only genes with nearly unaltered gene expression in the BAL were MIP-1 $\alpha$ and CXCR4 while in the lung samples these genes also showed increased expression. Interestingly, expression of the important mediators of monocytes and macrophages migration MCP-1 and MIP-1 $\alpha$ was strongly increased in lung samples from AAI mice and GC therapy led to further elevation. The expression of CXCR4 remained unchanged without any observable effect of AAI induction and GC therapy. This result was surprisingly because of the reported role for this chemokine receptor in the mobilization of hematopoietic stem cells into the bloodstream during an inflammatory response and its expression on $\mathrm{T}_{\mathrm{H}} 2$ cell subset. iNOS and IL-10 expression in BAL samples was nearly the same independent of the treatment. However, because the iNOS is a characteristic marker of the inflamed epithelium and activated macrophages it was interestingly to analyze its expression in the lung tissue. Based on the obtained data, the regulation of these two genes in the inflamed tissue was more critical for therapeutic efficiency of AAI as their regulation in the cellular infiltrate. The results of the iNOS gene expression analysis in lung tissue samples revealed a successful suppression of iNOS expression in samples of wild type mice while the expression of this inflammatory enzyme was even upregulated in GC-treated asthmatic GR ${ }^{\text {dim }}$ mice. IL-10 showed a very similar regulation as iNOS. In contrast to the expression of this cytokine in the BAL samples, the IL-10 expression in the lung samples was strongly increased after AAI induction. This overexpression of IL-10 in the inflamed lungs could be strongly suppressed in wild type mice by Dexamethasone treatment while the IL-10 mRNA levels in the lung samples of GC-treated $\mathrm{GR}^{\mathrm{dim}}$ mice was slightly upregulated. These data demonstrate that these two inflammatory mediators are more important in the inflamed tissue than in the infiltrating cells. Additionally, the expression of these two genes was strongly dependent on the DNA-binding dependent mode of GR action in the lungs. 


\begin{tabular}{|c|c|c|}
\hline Gene expression & BAL & Lung \\
\hline $\begin{array}{l}\text { No effects of GC } \\
\text { therapy/ unaltered } \\
\text { gene expression } \\
\text { in both genotypes }\end{array}$ & $\begin{array}{l}\text { CXCR4 } \\
\text { MIP-1 } \alpha \\
\text { MCP-1 } \\
\text { iNOS } \\
\text { IL-10 } \\
\text { CXCR3 }\end{array}$ & $\begin{array}{l}\text { CXCR4 } \\
\text { MIP-1 } \alpha \\
\text { MCP-1 }\end{array}$ \\
\hline $\begin{array}{c}\text { DNA-binding } \\
\text { dependent effect of } \\
\text { GC therapy } \\
\text { (no regulation in } \\
\text { GR } \text { dim mice) }^{\text {dim }}\end{array}$ & $\begin{array}{c}\text { IL-13 } \\
\text { IL-4 } \\
\text { RANTES }\end{array}$ & $\begin{array}{c}\text { IL-13 } \\
\text { IL-4 } \\
\text { RANTES } \\
\text { IL-5 } \\
\text { Eotaxin-2 } \\
\text { iNOS } \\
\text { IL-10 }\end{array}$ \\
\hline $\begin{array}{l}\text { DNA-binding } \\
\text { independent effect of } \\
\text { GC therapy } \\
\text { (regulation in both } \\
\text { genotypes) }\end{array}$ & $\begin{array}{c}\text { IL-5 } \\
\text { Eotaxin-2 }\end{array}$ & CXCR3 \\
\hline
\end{tabular}

Figure 63: Effects of GC therapy on gene expression of inflammatory mediators in BAL and lung samples of wild type and $\mathbf{G R}^{\mathrm{dim}}$ mice. The data represent an overview of all results obtained by RTqPCR analysis.

The induction of AAI led to an elevated expression of IL-5 and Eotaxin-2, the prominent markers of eosinophilia. This upregulation could be strongly suppressed by GC therapy in BAL cells from wild type and $\mathrm{GR}^{\mathrm{dim}}$ mice. Although GCs could reduce the expression of these genes in infiltrating cells in GR ${ }^{\mathrm{dim}}$ mice, the expression of IL-5 and Eotaxin-2 in lung parenchymal cells was not altered by Dexamethasone treatment. On the one hand, these results show that the observed lung infiltration with eosinophils (see FACS data) is more dependent on the cytokine and chemokine expression in lung cells. On the other hand these data demonstrate the differences in gene regulation by GCs between individual cell types. This ambivalent effect of GC therapy was also seen in the regulation of CXCR3 expression. While its expression in the BAL samples was nearly unaltered after GC therapy in both genotypes, the expression in lung tissue samples was reduced in wild type and $\mathrm{GR}^{\mathrm{dim}}$ mice. CXCR3 was preferentially reported to be expressed on activated $\mathrm{T}_{\mathrm{H}} 1$ cells, NK cells but also on epithelial and endothelial cells of the lungs. It was also reported, that the expression of this chemokine receptor can influence the migration of $\mathrm{T}_{\mathrm{H}} 2$ cells into the lungs and the activation 
of effector T cells. However, based on this result, the reduction of CXCR3 in the inflamed lung tissue by GCs did not prevent the strong infiltration seen in $\mathrm{GR}^{\mathrm{dim}}$ mice.

The expression of a number of key mediators of asthmatic reactions was influenced by GC therapy in a DNA-binding dependent mode of action. The elevated expression of IL-4 and IL-13 was proposed to play a crucial role in the pathogenesis of asthma by activating and recruiting a variety of inflammatory cells involved in inflammation. Increased IL-4 and IL-13 levels were observed in BAL and lung samples of AAI mice of both genotypes. The expression of IL-13 was suppressed in a DNA-binding dependent mode of action in BAL and lung samples. The expression of IL-4 in the lungs but not the BAL of wild type mice was increased because of GC therapy and suppressed in the BAL but not lung samples. These confusing effects could be based on a variety of factors including differences in the cell composition between BAL and lung tissue and possible dissimilarity in $T_{H} 2 / T_{H} 1$ balance regulation. The inflammatory mediator RANTES was strongly increased after AAI induction. RANTES was reported to be able to induce eosinophilia in the lungs independent of the presence of the allergen thereby increasing asthma severity in patients. The expression data demonstrated a strong suppression of RANTES after GC-therapy in samples of wild type mice with AAI. While the expression in allergic wild type mice was significantly downregulated, GC-therapy of $\mathrm{GR}^{\mathrm{dim}}$ mice did neither affect RANTES mRNA expression in BAL nor lung tissue samples. This observation showed a strong dependency of RANTES regulation on the DNA-binding dependent mode of GR action.

\subsubsection{Conclusion}

The results of this part of the work demonstrate that the therapeutic efficiency in the treatment of asthma with GCs is fully dependent on DNA-binding and dimerisation of the GR. In the absence of transactivation GC treatment could neither reduce the lung infiltration with eosinophils nor prevent airway remodeling and suppress gene expression of key mediators of asthmatic reaction. Furthermore, GC effects are neither critically dependent on the suppression of proliferation of splenocytes nor of IL-2, IL-4 or IFNy production. Also the unaltered antigen-specific immunoglobulin levels were irrelevant for therapeutic efficiency in wild type mice. Based on gene expression analysis of BAL and lung samples the following mediators appear to be important for the treatability of AAI with GCs: IL-4 and IL-13, IL-5 and Eotaxin-2 as well as iNOS and RANTES. 

AAI

Because the GR is present in almost every vertebrate cell, it is important to determine the crucial target cells, which are important for the anti-inflammatory effects of GCs during asthma therapy. To identify the essential target cells of GC therapy of an asthmatic reaction in this work cell-type specific GR-deficient mice which lack the GR in T cells (GR $\left.{ }^{\text {lck }}\right)$, myeloid cells $\left(\mathrm{GR}^{\mathrm{lys} \mathrm{M}}\right)$, DCs $\left(\mathrm{GR}^{\mathrm{CD} 11 \mathrm{C}}\right)$ or B cells $\left(\mathrm{GR}^{\mathrm{CD} 19}\right)$ were used.

The analysis of lung infiltration in wild type and mutant mice revealed very similar efficiencies of GC therapy in all mutant mouse strains. Dexamethasone treatment successfully reduced the cellular influx into the lungs of wild type and mutant mice independent on GRexpression in T cells, myeloid cells (including eosinophils), DCs or B cells. GC therapy did not only reduce the cell number in the BAL from mutant mice with AAI but also the alterations in the composition of the BAL were similar. Additional analysis of proliferation, cytokine production and immunoglobulin also secretion showed mostly the same regulation by GCs in mutant as in wild type mice. The finding that $\mathrm{GR}^{\text {lysM }}, \mathrm{GR}^{\text {lck }}, \mathrm{GR}^{\mathrm{CD} 11 \mathrm{C}}$ and $\mathrm{GR}^{\mathrm{CD} 19}$ mice are treatable with Dexamethasone indicates that suppression of eosinophils, macrophages and neutrophils, DCs, T and B cells is probably not essential for therapeutic efficiency. This indicated that the therapy efficiency was more dependent on the nonhematopoietic cells of the lungs than on the immune cells themseves.

The role of non-immune cells during the pathogenesis of asthma and its therapy is only partially understood. However, recent publications suggest that the structural cells of the lungs play an important function in disease initiation and progression. The non-immune cells like epithelial cells, fibroblasts, endothelial cells and smooth muscle cells not only build the lung structure and provide the gas exchange function but also produce a large amount of inflammatory and regulatory mediators. To investigate structural cells of the lungs are for GC-therapy, bone marrow chimeras with GC-refractory GR $^{\mathrm{dim}}$ mice were generated. Since $\mathrm{GR}^{\mathrm{dim}}$ mice are almost untreatable because of the absent DNA-binding mode of GR action, bone marrow chimeras with wild type or $\mathrm{GR}^{\mathrm{dim}}$ mice as recipient and wild type mice or $\mathrm{GR}^{\mathrm{dim}}$ mice as donors of bone marrow cells for the reconstitution of the immune system could be used to dissect the role of immune cells as opened to structural cells of the lungs for GCtherapy of asthma. The analyses of the therapeutic efficiency of GC therapy of the bone marrow chimeras showed that the effect of GC-treatment is dependent on the responsiveness 
of the non-immune cells. In spite of a wild type hematopoietic system bone marrow chimeras with $\mathrm{GR}^{\mathrm{dim}}$ mice as recipients were not treatable with Dexamethasone. This effect was observed by FACS analysis of the BAL concerning cell composition and absolute cell numbers. The AAI in the lungs of bone marrow chimeras with wild type mice as recipient could be successfully suppressed independent of the genotype of the hematopoietic system. The eosinophilia in the lungs of bone marrow chimeras with $\mathrm{GR}^{\mathrm{dim}}$ mice as recipient with both sorts of the reconstituted hematopoietic system was unaltered by GC therapy. The only observable difference in the reaction to GCs was observed in the regulation of proliferation and cytokine secretion of splenocytes ex vivo. Proliferation and IL-2 secretion were only reduced by GCs in samples of bone marrow chimera with wild type immune system and recipient mice. The chimeras with a $\mathrm{GR}^{\mathrm{dim}}$ immune system showed a slight increase in proliferation and IL-2 secretion in mice treated with Dexamethasone. The chimeras with wild type mice as recipients and $\mathrm{GR}^{\mathrm{dim}}$ mice as bone marrow donors showed only a small reduction of IL-2 production and unaltered proliferation despite the therapy. However, this small difference in the control of the splenocytes did not result in an observable effect on therapeutic efficiency.

Beside the structural cells of the lungs mast cells could be a key regulator of the inflammatory reaction. In contrast to other immune cells, which can be easily replaced via reconstitution of the hematopoietic system, most mast cells which reside in the tissue have a life span of several weeks or months and cannot be completely substituted from the donor bone marrow in irradiated recipient mice. Because mast cells are supposed to be a target cell of GCs, it was important to investigate the dependency of GC-therapy on the suppression of mast cells. Interestingly, the development and pathogenesis of an asthmatic reaction in cKit mice was similar to wild type mice. Furthermore, mast cell-deficient mice were fully treatable by GCs. Taken together, lung parenchymal cells but not mast cells appear to be the major target of GC-therapy of asthma in AAI mouse model.

\subsubsection{Effects of GC therapy on the regulation of gene expression in the lungs}

In order to further investigate the mechanisms of GC-therapy in $\mathrm{GR}^{\mathrm{dim}}$ bone marrow chimeras the expression of various important inflammatory mediators was analyzed by RT-qPCR. The analysis of gene expression in the lungs of GC treated bone marrow chimeras allowed to dissect the effects of GCs on immune cells and structural cells in the lungs. 
The expression of different genes that play important role in the pathogenesis of asthma was analyzed in order to find the critical ones for GC-therapy of an asthmatic reaction. The result of this analysis showed that the respective genes can be classified into three groups.

The first group of genes showed strong a dependency on the treatability of the immune system (Figure 64). The effects of GCs in this group correlated with the genotype of the bone marrow but not with genotype of the recipient mice. These genes included $\beta$-Tryptase, Eotaxin-2, RANTES, IL-13, IL-5 and Occludin. Wild type mice reconstituted with GR ${ }^{\text {dim }}$ bone marrow did not show suppression of these genes while $\mathrm{GR}^{\mathrm{dim}}$ chimeras with wild type bone marrow showed nearly the same gene regulation as wild type chimeras. Although the genes in this group are known as activation markers and important regulators of eosinophilia, induction of a $\mathrm{T}_{\mathrm{H}} 2$ response and barrier function of the lungs, they do not seem to be critical for the suppression of inflammation by GCs during asthma treatment. Although these genes in $\mathrm{GR}^{\mathrm{dim}}$ chimeras with a wild type immune system were regulated in the same manner as in wild type chimeras with a wild type immune system was not accompanied by similar GC effects on lung infiltration.

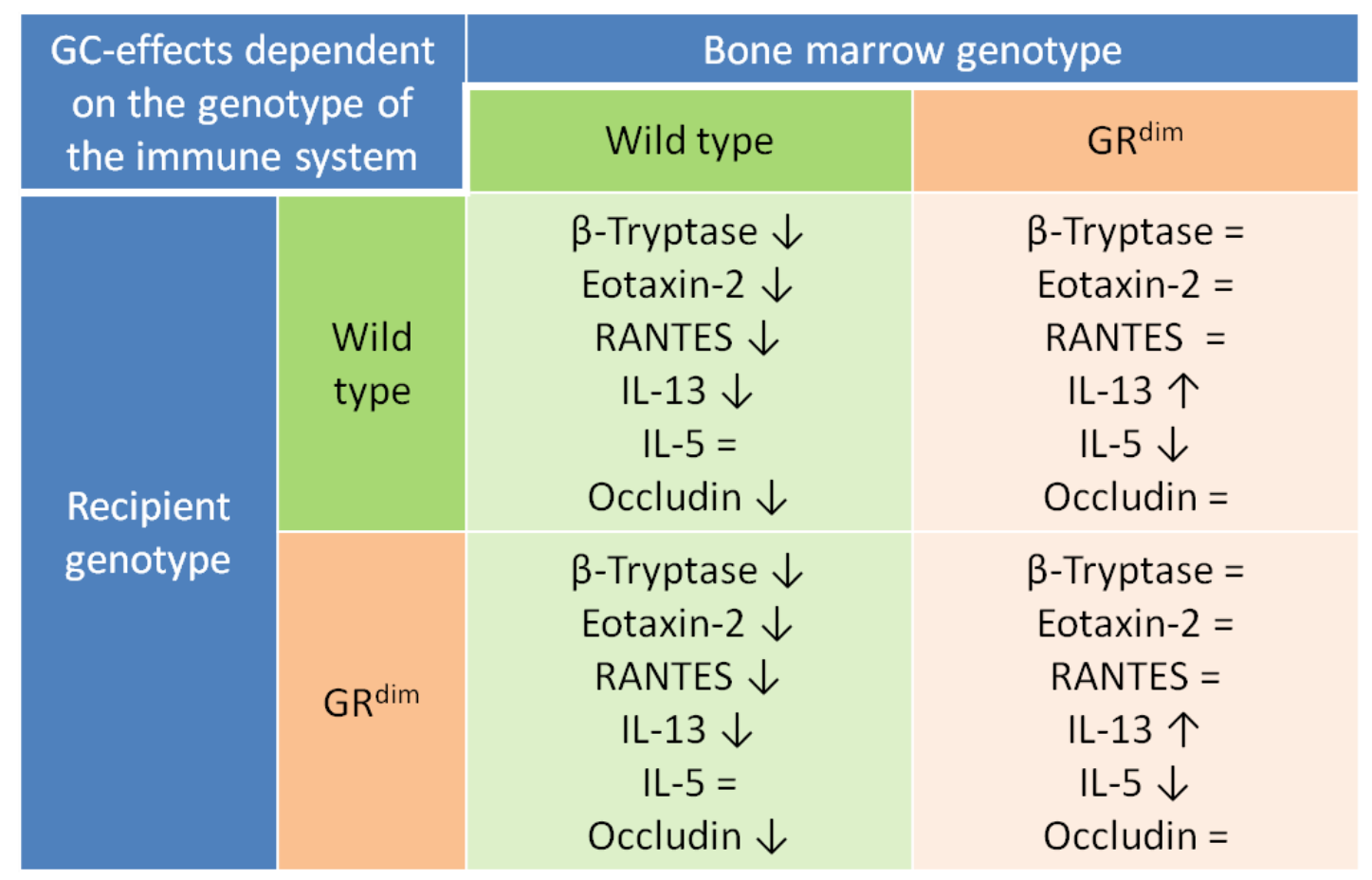

Figure 64: Immune system dependent GC-effects on gene expression in lung tissue of wild type and $G^{\mathrm{dim}}$ bone marrow chimeras reconstituted with wild type or $\mathbf{G R}^{\mathrm{dim}}$ bone marrow. The data represent an overview of the results obtained by RT-qPCR analysis. 
The second group of genes showed a strong dependency on the treatability of the lung structural cells. These genes could be suppressed by GC therapy only in chimeras with wild type mice as recipients independent of the genotype of the reconstituted immune system. These genes include IL-9, TSLP, IL-25, IL-33 and IL-10.

The genes, the expression of which showed dependency on the responsiveness of structural cells of the lungs, are known as important regulators of the inflammatory response. GC therapy of wild type chimeras led to a reduction of IL-9, TSLP, IL-25 and IL-10 expression irrespective of the genotype of the bone marrow while the expression of IL-33 was upregulated. In contrast, these genes were not altered by GC-therapy in all bone marrow chimeras with $\mathrm{GR}^{\mathrm{dim}}$ mice as recipients.

The suppression of IL-9 could play an important role in the treatment of asthmatic reaction because this cytokine influences mast cell development and activation and also drives mucus production by a direct influence on airway epithelia and also by interacting with IL-13. Additionally IL-9 plays a role in airway remodeling and immune-cell recruitment into the lungs by promoting inflammatory mediators release such as Eotaxin-2.

The expression of TSLP was reported to be a critical link between the initial epithelial response and the development of airway inflammation (Holgate, 2007). This cytokine is produced by epithelial cells and capable to direct DCs towards $\mathrm{T}_{\mathrm{H}} 2$ response. Additionally TSLP can directly influence mast cell activation and a $\mathrm{T}_{\mathrm{H}} 2$ cytokine production. Secretion of TSLP is increased in the epithelium of asthmatic patients and correlates with disease severity. The data from the analysis of TSLP expression in the lung of bone marrow chimera demonstrated that therapeutic effectiveness and TSLP regulation correlated.

IL-25 is also a cytokine that promotes $\mathrm{T}_{\mathrm{H}} 2$ immune responses in asthmatic reactions at mucosal epithelial surfaces. This cytokine was reported to be able to initiate an asthmatic reaction with goblet cell hyperplasia, eosinophilia and IL-9 production independent of IL-4. The suppression of IL-25 expression could only be seen in animals with wild type mice as a recipient. Thus, downregulation of IL-25 by GCs and treatability correlate, suggesting a causal relationship of gene regulation and asthma therapy. 


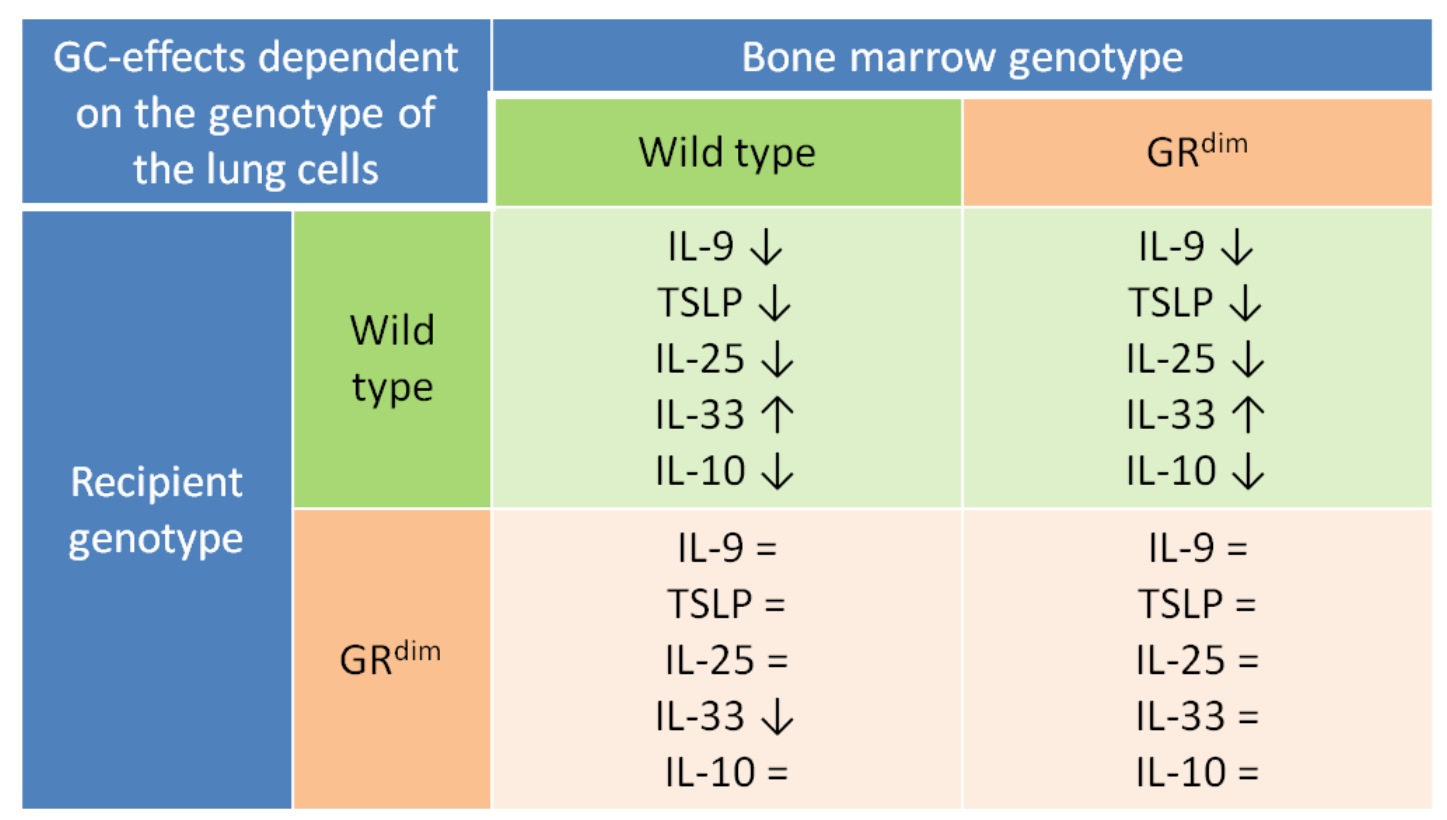

Figure 65: Lung cells dependent GC-effects on gene expression in lung tissue of wild type and GR $^{\mathrm{dim}}$ bone marrow chimeras reconstituted with wild type or $\mathbf{G R}^{\mathrm{dim}}$ bone marrow. The data represent an overview of the results obtained by RT-qPCR analysis.

The function of IL-10 in asthma pathogenesis is not completely understood. This cytokine was reported to have an anti-inflammatory function because of the inhibition of $\mathrm{T}_{\mathrm{H}} 2$ cytokine production via IL-10 secreting T reg cells (Robinson et al., 2004; Xystrakis et al., 2006). Beside $\mathrm{T}$ reg cells also alveolar macrophages and mast cells can produce this cytokine in the lungs. The reduction of mRNA expression therefore could be a sign of the downregulation of mast cell activity by GCs.

The regulation of the last cytokine in this group- IL-33- is complex. It is known, that it plays a crucial role in the initiation of an asthmatic reaction. This cytokine is one of the earliestreleased signaling molecules following epithelial damage in the lungs. Its enhanced expression leads to the activation of $\mathrm{T}_{\mathrm{H}} 2$ cells, DCs, mast cells and eosinophils and the secretion of a variety of inflammatory mediators. The expression of IL-33 was upregulated in the samples of GC treated chimeras when wild type mice were used as recipients. This effect of GC therapy was surprising but is in line with data from asthmatic patients as reported by Prefontaine and colleagues. They demonstrated that the expression of IL-33 originates from structural cells of the airways and that it does not respond to classical anti-inflammatory drugs used to treat asthma (Prefontaine et al., 2010). 
The third group of genes included those with a complex regulation pattern. The expression of iNOS and CXCR4 was reduced by GC treatment in all types of bone marrow chimeras while the expression of IL-4 was upregulated. CXCL12 expression could be suppressed in all chimeras excluding wild type chimeras with a $\mathrm{GR}^{\mathrm{dim}}$ immune system. CXCR3 demonstrated was upregulated after Dexamethasone treatment in all chimeras excluding wild type chimera with a wild type immune system. This data shows another facet of complexity and divergence of the factors that influence the therapeutic efficiency of GCs.

Based on the results of the gene expression analysis of lung samples from $\mathrm{GR}^{\mathrm{dim}}$ bone marrow chimeras GC therapy seems to be largely dependent on the suppression of characteristical genes expressed by inflamed lung cells. The suppression of IL-9, IL-10, TSLP and IL-25 by GCs and treatability strongly correlate, suggesting a key role of these mediators in the asthma pathogenesis. These mediators such as TSLP and IL-25 are good candidates for important targets of GC-therapy in the treatment of asthma. The regulation of other genes that were reported to be markers for mast cell activation ( $\beta$-Tryptase), myeloid cell migration and activation (MCP-1, Mip-1 $\alpha$, iNOS), eosinophilia (IL-5, Eotaxin-2) and T cell priming (IL-4, IL-13, RANTES) did not directly correlate with therapeutic efficiency, suggesting a secondary role of these cytokines and chemokines in regulation of inflammatory response by GCs.

\subsubsection{Role of airway epithelial cells for GC-therapy of asthma in inducible GR $^{\text {SPCcreERT2 }}$ mice}

The possibility to treat mice lacking the GR in T and B, DCs and myeloid cells, the experiments with mast cell deficient mice and bone marrow chimeras suggested that nonimmune cells (like epithelial cells of the lung) play an important role in the suppression of inflammatory reactions by GCs during asthma therapy.

To investigate the role of the epithelial cells as targets of GC-therapy inducible GR ${ }^{\text {SPCreERT2 }}$ mice were used in this work. Because of the efficiently diminished expression of the GR in AT2 epithelial cells and the normal lung physiology of these mutant mice they could be used to investigate this issue without limitations. While the pathogenesis of the asthmatic reaction was similar compared to wild type animals, the analysis of the therapeutic efficiency showed that the treatability of the mutant mice was impaired. The cellular infiltration of the mutant lungs was only partially reduced after GC therapy. The analysis of the BAL cell composition 
demonstrated almost the same level of eosinophilia in samples of $\mathrm{GR}^{\text {SPCcreERT2 }}$ with or without treatment. Based on these data, the AT2 cells play a crucial role during asthma therapy with GCs.

Gene expression analysis of sorted AT2 cells was done to identify regulatory mechanisms of GCs in the treatment of asthma.

\begin{tabular}{|c|c|c|}
\hline Effect of GCS & GR $^{\text {flox }}$ & GR $^{\text {SPCcreERT2 }}$ \\
\hline $\begin{array}{c}\text { Upregulation of } \\
\text { mRNA expression }\end{array}$ & ZO-1 & IL-1 $\beta$, IL-33 \\
\hline IP-10 & iNOS \\
Occludin & TSLP \\
Occludin
\end{tabular}

Figure 66: Effects of GC therapy on gene expression in AT2 cells isolated from Dexamethasone treated AAI mice of wild type and $\mathbf{G R}^{\text {SPCreERT2 }}$ genotype. The data represent an overview of the results obtained by RT-qPCR analysis.

Current publications reported an important role of pulmonary epithelial cells during lung infection and a critical role of epithelial cells in the initiation of an asthmatic reaction. An important role in this process was assigned to the TLR-mediated pathway. After contact with microbes or microbial products, TLR-mediated signals result in the production of TNF- $\alpha$, IL-1 $\beta$, TSLP and IL-33 by epithelial cells. These cytokines stimulate the expression of adhesions molecules on vascular endothelial cells, activate DCs and promote a $\mathrm{T}_{\mathrm{H}} 2$ response, initiating an asthmatic reaction. Several publications point towards TLR as a possible target for a more specific asthma therapy. The data from the initial analysis of $\mathrm{GR}^{\text {SPCcreERT2 }}$ mice revealed a strong upregulation of mediators that are correlated with TLR-signals and diminished effects of GC-therapy on their downregulation compared to the wild type samples. Although these results represent only a first data set a correlation between the treatability of the mice and the expression pattern of AT2 cells could be seen. The regulation of all analysed genes by GC treatment was different in mutant mice compared to wild type samples. While 
GCs in wild type animals successfully downregulated the mRNA expression of critical inflammatory mediators like IL-1 $\beta$, IL-33 and iNOS these genes showed increased expression in samples of GR ${ }^{\text {SPCcreERT2 }}$ mice. The expression of TSLP was increased in AT2 samples of GC-treated $\mathrm{GR}^{\mathrm{SPC} \text { PCEERT2 }}$ mice while the expression of other important mediator like RANTES, Eotaxin-2 and TNF- $\alpha$ reminded unaltered independent on GC therapy. The barrier function of epithelial cells was impaired in both genotypes after induction of AAI but only in wild type mice the GC treatment led to an increased expression of ZO-1. The upregulation of ZO-1 expression is reported to be a sign of an improvement of the epithelial barrier function during the therapy and is important to closer contact of DCs with the environment.

Based on the initial observations, the following genes could be key players for the therapeutic efficiency of GCs: ZO-1 (important for the barrier function); TNF $\alpha$, IL-1 $\beta$ and IL-33 (initiator and mediators of inflammatory response), Eotaxin-2 and RANTES (mediators of cellular infiltration) and TSLP (activation of DCs and initiation of asthmatic reaction). A possible explanation of the observed effects could be the regulation of TLR signalling by GCs, which could lead to a strong inhibition of DCs maturation in the lungs. The fact that the recruitment, activation and intraepithelial migration of DCs in response to microbial components as inhaled endotoxin requires only triggering of epithelial TLR-4 (Hammad H. et al., 2009) demonstrates how important the interaction between the immune system and epithelial cells and effect of GC treatment on the epithelial cells activation is. Finally, mast cell activation and subsequent secretion of proinflammatory mediators is directly induced by TLR ligands as well and can be regulated by GCs via effects on TLR expression.

\subsubsection{Conclusion}

The experimental data obtained with cell type specific GR knockout mutant mice and GR ${ }^{\text {dim }}$ bone marrow chimeras suggest a crucial role of non-immune cells of the lungs during the therapy of asthmatic reactions with GCs. The loss of GR expression in T cells, myeloid cells, DCs and B cells did not result in an impaired therapeutic efficiency of GCs. Also when the immune system did not respond to GCs, inflammation could still be suppressed by GCs in bone marrow chimera with wild type mice as recipients. The data on GR ${ }^{\text {SPCreERT2 }}$ mice further support the hypothesis that AT2 epithelial cells are important targets of GCs during asthma treatment. Based on the gene expression analysis and current publications in field, AT2 cells seems to play a key role in the development of asthma and its pathogenesis and might be critical for the therapeutic efficiency of GCs. 
Adcock IM, Barnes PJ. Molecular Mechanisms of Corticosteroid Resistance. Chest. 2008;134(2):394-401.

Aikawa T, Shimura S, Sasaki H, Ebina M, Takishima T. Marked goblet cell hyperplasia with mucus accumulation in the airways of patients who died of severe acute asthma attack. Chest 1992; 101: 916-921.

Alam R. Chemokines in allergic inflammation. J Allergy Clin Immunol. 1997; 99:273-7.

Alam, R., York, J., Boyars, M., Stafford, S., Grant, J. A., Lee, J., Forsythe, P., Sim, T. and Ida, N., Increased MCP-1, RANTES, and MIP-1alpha in bronchoalveolar lavage fluid of allergic asthmatic patients. Am. J. Respir. Crit. Care Med. 1996. 153: 1398-1404.

Araya J, Nishimura SL. Fibrogenic reactions in lung disease. Annu Rev Pathol. 2010; 5:7798

Andrews RC, Walker BR. Glucocorticoids and insulin resistance: old hormones, new targets. Clin Sci (Lond). 1999 May;96(5):513-23.

Austen J M, Gordon S.F4/80, a monoclonal antibody directed specifically against mouse macrophages. Eur. J. Immunol.1981: 11, 805-815

Balhara and Gounni AS. The alveolar macrophages in asthma: a double-edged sword; Mucosal Immunology 2012: 5, 605-609

Ballantyne SJ, Barlow JL, Jolin HE, Nath P, Williams AS, Chung KF, Sturton G, Wong SH, McKenzie AN.. Blocking IL-25 prevents airway hyperresponsiveness in allergic asthma. J. Allergy Clin. Immunol. 2007: 120:1324-1331.

Barnes PJ, Adcock IM. How Do Corticosteroids Work in Asthma? Ann Intern Med. 2003;139(5_Part_1):359-70.

Barnes PJ. Glucocorticosteroids: current and future directions. $\mathrm{Br} \mathrm{J}$ Pharmacol. 2011 May;163(1):29-43.

Barrett NA \& Austen KF. Innate cells and T helper 2 cell immunity in airway inflammation. Immunity 2009: 31, 425-437.

Baschant U, Frappart L, Rauchhaus U, Bruns L, Reichardt, HM, Kamradt T, Bräuer R, and Tuckermann JP. Glucocorticoid therapy of antigen-induced arthritis depends on the dimerized glucocorticoid receptor in $\mathrm{T}$ cells. Proceedings of the National Academy of Sciences USA 2011: 108, 19317-19322. 
Baumann S, Dostert A, Novac N, Bauer A, Schmid W, Fas SC, Krueger A, Heinzel T, Kirchhoff S, Schütz G, Krammer PH. Glucocorticoids inhibit activation-induced cell death (AICD) via direct DNA-dependent repression of the CD95 ligand gene by a glucocorticoid receptor dimer. Blood. 2005 Jul 15;106(2):617-25.

Beato M, Herrlich P, Schütz G. Steroid hormone receptors: many actors in search of a plot. Cell. 1995 Dec 15;83(6):851-7.

Beato M, Klug J. Steroid hormone receptors: an update. Hum Reprod Update. 2000 MayJun;6(3):225-36.

Belz GT, Smith CM, Kleinert L, Reading P, Brooks A, Shortman K, Carbone FR, and Heath. WR. Distinct migrating and nonmigrating dendritic cell populations are involved in MHC class Irestricted antigen presentation after lung infection with virus. Proc. Natl. Acad. Sci. USA 2004: 101:8670-8675.

Berry MA, Hargadon B, Shelley M, Parker D, Shaw DE, Green RH, Bradding P, Brightling CE, Wardlaw AJ, Pavord ID.. Evidence of a role of tumor necrosis factor alpha in refractory asthma. N. Engl. J. Med. 2006: 354:697-708.

Bhattacharyya S, Brown DE, Brewer JA, Vogt SK, Muglia LJ. Macrophage glucocorticoid receptors regulate Toll-like receptor 4-mediated inflammatory responses by selective inhibition of p38 MAP kinase. Blood. 2007;109:4313-4319

Blease K. Therapeutics targeting IL-13 for the treatment of pulmonary inflammation and airway remodeling. Curr Opin Investig Drugs 2008: 9(11):1180-4.

Bice D. E., Seagrave J., and Green F.H. (2000) Animal models of asthma: potential usefulness for studying health effects of inhaled particles. Inhal. Toxicol. 12: 829-862

Borish L and Steinke JW, Interleukin-33 in Asthma: How Big of a Role Does It Play? Curr Allergy Asthma Rep. 2011 February; 11(1): 7-11.

Bousquet, J., Jeffery, P. K., Busse, W. W., Johnson, M. and Vignola, A. M. (2000). Asthma from bronchoconstriction to airways inflammation and remodelling. Am. J. Respir. Crit. Care Med. 161, 1720-1745

Boyce, J. A. and Austen, K. F. (2005). No audible wheezing: nuggest and conundrums from mouse asthma models. J. Exp. Med. 201, 1869-1873

Buttgereit F, Scheffold A. Rapid glucocorticoid effects on immune cells. Steroids. 2002 May;67(6):529-34.

Caelles C, Gonzalez-Sancho JM, Munoz A. Nuclear hormone receptor antagonism with AP-1 by inhibition of the JNK pathway. Genes Dev 11,1997: 3351-3364.

Caton ML, Smith-Raska MR, Reizis B. Notch-RBP-J signaling controls the homeostasis of CD8- dendritic cells in the spleen. J Exp Med 2007: 204:1653-1664. 
Carrol NG, Cooke C, James AL. Bronchial blood vessel dimensions in asthma. Am J Respir Crit Care Med 1997; 155: 689-695

Cheung J, Smith DF. Molecular chaperone interactions with steroid receptors: an update. Mol Endocrinol. $2000 \mathrm{Jul} ;$ 14(7):939-46.

Cole TJ, Blendy JA, Monaghan AP, Krieglstein K, Schmid W, Aguzzi A, Fantuzzi G, Hummler E, Unsicker K, Schütz G. Targeted disruption of the glucocorticoid receptor gene blocks adrenergic chromaffin cell development and severely retards lung maturation. Genes Dev. 1995 Jul 1; 9(13):1608-21.

Corrigan JC. Eotaxin and asthma: some answers, more questions. Clin Exp Immunol. 1999 April; 116(1): 1-3.

Chung KF, Patel HJ, Fadlon EJ, Rousell J, Haddad EB, Jose PJ, Mitchell J, Belvisi M:Induction of eotaxin expression and release from human airway smooth muscle cells by IL1beta and TNFalpha: effects of IL-10 and corticosteroids. Br J Pharmacol 1999: 127(5):11451150.

Czar MJ, Lyons RH, Welsh MJ, Renoir JM, Pratt WB. Evidence that the FK506-binding immunophilin heat shock protein 56 is required for trafficking of the glucocorticoid receptor from the cytoplasm to the nucleus, Mol Endocrinol 9(11) 1995: 1549-1560.

De S, Zelazny ET, Souhrada JF, Souhrada M. Il-1b and Il-6 induce hyperplasia and hypertrophy of cultured guinea pig airway smooth muscle cells. J Appl Physiol 1995; 78: $1555-1563$.

Denis M, Gustafsson JA, Wikstrom AC. Interaction of the $\mathrm{Mr}=90,000$ heat shock protein with the steroid-binding domain of the glucocorticoid receptor, J Biol Chem 263(34) 1988: 18520-18523.

Dong Q, Louahed J, Vink A, Sullivan CD, Messler CJ, Zhou Y, Haczku A, Huaux F, Arras M, Holroyd KJ, Renauld JC, Levitt RC, Nicolaides NC.. IL-9 induces chemokine expression in lung epithelial cells and baseline airway eosinophilia in transgenic mice. Eur. J. Immunol. $1999 \mathrm{Jul} ; 29(7): 2130-9$

Dorman SC, Babirad I, Post J, Watson RM, Foley R, Jones GL, O'Byrne PM, Sehmi R.. Progenitor egress from the bone marrow after allergen challenge: role of stromal cell-derived factor 1alpha and eotaxin. J. Allergy Clin. Immunol. 2005: 115:501-507.

Dunnill M, Massarella G, Anderson J. Comparison of the quantitative anatomy of the bronchi in normal subjects, in status asthmaticus, in chronic bronchitis and in emphysema. Thorax 1969; 24: 176-179.

Eklund, K.K., Ghildyal, N., Austen, K.F., and Stevens, R.L. Induction by IL-9 and suppression by IL-3 and IL-4 of the levels of chromosome 14-derived transcripts that encode late-expressed mouse mast cell proteases. J. Immunol. 1993; 151:4266-4273. 
Erin EM, Leaker BR, Nicholson GC, Tan AJ, Green LM, Neighbour H, Zacharasiewicz AS, Turner J, Barnathan ES, Kon OM, Barnes PJ, Hansel TT. The effects of a monoclonal antibody directed against tumour necrosis factor-alpha in asthma. Am. J. Respir. Crit. Care Med. 2006: 174:753-762.

Feil R, Wagner J, Metzger D, Chambon P. Regulation of Cre recombinase activity by mutated estrogen receptor ligand-binding domains. Biochem Biophys Res Commun. 1997 Aug 28;237(3):752-7.

Folkerts G, Nijkamp FP. Airway epithelium: more than just a barrier! Trends Pharmacol Sci. 1998 Aug;19(8):334-41.

Fort MM, Cheung J, Yen D, Li J, Zurawski SM, Lo S, Menon S, Clifford T, Hunte B, Lesley R, Muchamuel T, Hurst SD, Zurawski G, Leach MW, Gorman DM,Rennick DM.. IL-25 induces IL-4, IL-5, and IL-13 and Th2-associated pathologies in vivo. Immunity. 2001: 15:985-995.

Förster C, Waschke J, Burek M, Leers J, Drenckhahn D. Glucocorticoid effects on mouse microvascular endothelial barrier permeability are brain specific. J Physiol. 2006 Jun 1;573(Pt 2):413-25.

Freedman ND, Yamamoto KR. Importin 7 and importin alpha/importin beta are nuclear import receptors for the glucocorticoid receptor. Mol Biol Cell. 2004 May;15(5):2276-86.

Frigas E, Gleich GJ. The eosinophil and the pathophysiology of asthma. J Allergy Clin Immunol 1986: 77(4):527-37.

Fryer AA, Spiteri MA, Bianco A, Hepple M, Jones PW, Strange RC, Makki R, Tavernier G, Smilie FI, Custovic A, Woodcock AA, Ollier WE. and Hajeer AH. The -403 G 1 A promoter polymorphism in the RANTES gene is associated with atopy and asthma. Genes Immun. 2000. 1: 509-514.

Fukakusa M, Bergeron C, Tulic MK, Fiset PO, Al Dewachi O, Laviolette M, Hamid Q, Chakir J. Oral corticosteroids decrease eosinophil and CC chemokine expression but increase neutrophil, IL-8, and IFN-gamma-inducible protein 10 expression in asthmatic airway mucosa. J Allergy Clin Immunol. 2005: Feb;115(2):280-6.

Ghaffar O, Hamid Q, Renzi PM, Allakhverdi Z, Molet S, Hogg JC, Shore SA, Luster AD, Lamkhioued B. Constitutive and cytokine-stimulated expression of eotaxin by human airway smooth muscle cells. Am J Respir Crit Care Med. 1999 Jun;159(6):1933-42.

Gounni AS, Gregory B, Nutku E, Aris F, Latifa K, Minshall E, North J, Tavernier J, Levit R, Nicolaides N, Robinson D, Hamid Q. Interleukin-9 enhances interleukin-5 receptor expression, differentiation, and survival of human eosinophils. Blood. 2000; 96:2163-2171. 
Greenfeder S, Umland SP, Cuss FM, Chapman RW and Egan RW. Th2 cytokines and asthma - The role of interleukin-5 in allergic eosinophilic disease. Respiratory Research 2001: 2:71-79

Green S, Kumar V, Theulaz I, Wahli W, and Chambon P. The N-terminal DNA-binding 'zinc finger' of the oestrogen and glucocorticoid receptors determines target gene specificity. EMBO J. 1988 October; 7(10): 3037-3044.

Grimbaldeston MA, Chen CC, Piliponsky AM, Tsai M, Tam SY, Galli SJ. Mast cell-deficient $\mathrm{W}$-sash c-kit mutant Kit $\mathrm{W}$-sh/W-sh mice as a model for investigating mast cell biology in vivo. Am J Pathol. 2005 Sep;167(3):835-48.

Hammad, H, Plantinga M, Deswarte K, Pouliot P, Willart MAM, Kool M, Muskens F, and Lambrecht BN. Inflammatory dendritic cells_ not basophils — are necessary and sufficient for induction of Th2 immunity to inhaled house dust mite allergen. J. Exp. Med. 2010: 207:20972111.

Hammad H, Chieppa M, Perros F, Willart MA, Germain RN, Lambrecht BN. House dust mite allergen induces asthma via Toll-like receptor 4 triggering of airway structural cells. Nat Med. 2009 Apr;15(4):410-6.

Harkema JR, Mariassy AST, Geroge J, Hyde DM, Plopper CG. Epithelial cells of the conducting airways: a species comparison. In: Farmer SG, Hay DWP, editors. The Airway Epithelium: Physiology, Pathology, and Pharmacology.1991: Vol. 55. New York: Marcel Dekker, Inc; pp. 3-39.

Herrick CA\& Bottomly K. To respond or not to respond: T cells in allergic asthma. Nature Reviews Immunology 2003: 3, 405-412

Hickson J. In vivo optical imaging: preclinical applications and considerations. Urol Oncol. 2009 May-Jun;27(3):295-7.

Hirase T, Staddon J, Saitou M, Ando-Akatsuka Y, Itoh M, Furuse M, Fujimoto K, Tsukita S, Rubin L. Occludin as a possible determinant of tight junction permeability in endothelial cells. J Cell Sci. 1997;110:1603-1613.

Hogan SP, Mould A, Kikutani H, Ramsay AJ, Foster PS. Aeroallergen-induced eosinophilic inflammation, lung damage, and airways hyperreactivity in mice can occur independently of IL-4 and allergen-specific immunoglobulins. J Clin Invest. 1997 Mar 15;99(6):1329-1339.

Holgate ST. Stratified approaches to the treatment of asthma. British Journal of Clinical Pharmacology. 2013: Volume 76, Issue 2, pages 277-291.

Holgate ST. Innate and adaptive immune responses in asthma. Nat Med. 2012 May 4;18(5):673-83.

Holgate ST. Epithelium dysfunction in asthma. J Allergy Clin Immunol. 2007 Dec;120(6):1233-44; 
Holgate ST. A Look at the Pathogenesis of Asthma: The Need for a Change in Direction. Discov Med. 2010 May;9(48):439-47.

Holgate ST, Polosa R. The mechanisms, diagnosis, and management of severe asthma in adults. Lancet. 2006: 368(9537):780-93

Holgate ST, Davies DE. Rethinking the pathogenesis of asthma. Immunity . 2009: 31(3):3627.

Holgate ST, Roberts G, Arshad HS, Howarth PH, Davies DE. The role of the airway epithelium and its interaction with environmental factors in asthma pathogenesis. Proc Am Thorac Soc. 2009: 6(8):655-9.

Holgate ST, Arshad HS, Roberts GC, Howarth PH, Thurner P, Davies DE. A new look at the pathogenesis of asthma. Clin Sci (Lond) 2009: 118(7):439-50

Holgate ST, Arshad HS, Roberts GC, Howarth PH, Thurner P, Davies DE. A new look at the pathogenesis of asthma. Clin Sci (Lond). 2009 Dec 23;118(7):439-50.

Holt PG. Antigen presentation in the lung. Am. J. Respir. Crit. Care Med. 2000: 162, S151S156.

Howard KJ, Holley SJ, Yamamoto KR, Distelhorst CW. Mapping the HSP90 binding region of the glucocorticoid receptor, J Biol Chem 265(20) 1990: 11928-11935.

Humbert, M., Ying, S., Corrigan, C., Menz, G., Barkans, J., Pfister, R., Meng, Q., Van Damme, J., Opdenakker, G., Durham, S. R. and Kay, A. B., Bronchial mucosal expression of the genes encoding chemokines RANTES and MCP-3 in symptomatic atopic and nonatopic asthmatics: relationship to the eosinophil-active cytokines interleukin (IL)-5, granulocyte macrophage-colony-stimulating factor, and IL-3. Am. J. Respir. Cell Mol. Biol. 1997. 16: 18 .

Innes AL, Carrington SD, Thornton DJ, Kirkham S, Rousseau K, Dougherty RH, Raymond WW, Caughey GH, Muller SJ, Fahy JV. Ex vivo sputum analysis reveals impairment of protease-dependent mucus degradation by plasma proteins in acute asthma. Am J Respir Crit Care Med. 2009 Aug 1;180(3):203-10.

Ito K, Chung KF, Adcock IM. Update on glucocorticoid action and resistance. J Allergy Clin Immunol. 2006;117(3):522-43.

Jabara HH, Brodeur SR, Geha RS. Glucocorticoids upregulate CD40 ligand expression and induce CD40L-dependent immunoglobulin isotype switching. J Clin Invest 2001. 107:371378.

Jackson DJ, Gangnon RE, Evans MD, Roberg KA, Anderson EL, Pappas TE, Printz MC, Lee WM, Shult PA, Reisdorf E, Carlson-Dakes KT, Salazar LP, DaSilva DF, Tisler CJ, Gern JE, Lemanske RF Jr. Wheezing rhinovirus illnesses in early life predict asthma development in high-risk children. Am J Respir Crit Care Med. 2008: 178(7):667-72. 
Kellendonk C, Tronche F, Monaghan AP, Angrand PO, Stewart F, Schutz G. Regulation of Cre recombinase activity by the synthetic steroid RU 486. Nucleic Acids Res1996; 24: 14041411.

Kino T, Souvatzoglou E, De Martino MU, Tsopanomihalu M, Wan Y, Chrousos GP. Protein 14-3-3sigma interacts with and favors cytoplasmic subcellular localization of the glucocorticoid receptor, acting as a negative regulator of the glucocorticoid signaling pathway. J Biol Chem 2003:278, 25651-25656.

Kips JC, Tavernier JH, Joos GF, Peleman RA, Pauwels RA. The potential role of tumor necrosis factor $\alpha$ in asthma. Clin. Exp. Allergy. 1993: 23:247-250.

Kips J. C., Anderson G.P., Fredberg U., Herz M. D., Schmidt P. J., Sterk A. J. M., Chung K.F. Murine models of asthma. Eur. Respir. J.2003: 22: 374-382

Kleiman A, Tuckermann JP. Glucocorticoid receptor action in beneficial and side effects of steroid therapy: lessons from conditional knockout mice. Mol Cell Endocrinol. 2007 Sep 15;275(1-2):98-108.

Knight DA, Holgate ST. The airway epithelium: structural and functional properties in health and disease. Respirology. 2003 Dec;8(4):432-46.

Kreider T, Anthony RM, Urban Jr JF, Gause WC. Alternatively activated macrophages in helminth infections. Current Opinion in Immunology.2007;19:448-453.

Korf JE, Pynaert G, Tournoy K, Boonefaes T, Van Oosterhout A, Ginneberge D, Haegeman A, Verschoor JA, De Baetselier P, and Grooten J. Macrophage Reprogramming by Mycolic Acid Promotes a Tolerogenic Response in Experimental Asthma. Am J Respir Crit Care Med 2006: Vol 174. pp 152-160.

Korideck H, Peterson JD. Noninvasive quantitative tomography of the therapeutic response to dexamethasone in ovalbumin-induced murine asthma. J Pharmacol Exp Ther. 2009 Jun;329(3):882-9.

Kumar RK, Herbert C and Foster PS. The „classical“"ovalbumin challenge model of asthma in mice. Curr. Drug Targets 2008: 9, 485-494

Lambrecht BL\& Hammad H. The airway epithelium in asthma. Nature Medicine. 2012: 18, 684-692

Lazaar AL, Panettieri RA Jr: Airway smooth muscle as an immunomodulatory cell: a new target for pharmacotherapy? Curr Opin Pharmacol 2001, 1(3):259-264.

Leonard WJ. TSLP: finally in the limelight. Nature Immunology 2002: 3, 605 - 607

Limbourg FP, Liao JK. Nontranscriptional actions of the glucocorticoid receptor. J Mol Med 2003: 81, 168-174 
Llewellyn BD. Nuclear staining with alum hematoxylin. Biotech Histochem. 2009;84(4):15977.

Longphre M, Li D, Gallup M, Drori E, Ordoñez CL, Redman T, Wenzel S, Bice DE, Fahy JV, Basbaum C.. Allergen-induced IL-9 directly stimulates mucin transcription in respiratory epithelial cells. J. Clin. Invest.1999: 104:1375-1382

Louahed, J., Kermouni, A., Van Snick, J., and Renauld, J.C. IL-9 induces expression of granzymes and high-affinity $\mathrm{IgE}$ receptor in murine $\mathrm{T}$ helper clones. J. Immunol. 1995: 154:5061-5070

Louahed J, Toda M, Jen J, Hamid Q, Renauld JC, Levitt RC, Nicolaides NC.. Interleukin-9 upregulates mucus expression in the airways. Am. J. Respir. Cell Mol. Biol. 2000: 22:649656

Louahed J, Zhou Y, Maloy WL, Rani PU, Weiss C, Tomer Y, Vink A, Renauld J, Van Snick J, Nicolaides NC, Levitt RC, Haczku A.. Interleukin 9 promotes influx and local maturation of eosinophils. Blood. 2001: 97:1035-1042.

Löwenberg M, Stahn C, Hommes DW, Buttgereit F. Novel insights into mechanisms of glucocorticoid action and the development of new glucocorticoid receptor ligands. Steroids. 2008;73(9-10):1025-9.

Luisi BF, Xu WX, Otwinowski Z, Freedman LP, Yamamoto KR, Sigler PB Crystallographic analysis of the interaction of the glucocorticoid receptor with DNA. Nature. 1991 Aug 8;352(6335):497-505.

Lukacs NW, Berlin A, Schols D, Skerlj RT, Bridger GJ. AMD3100, a CxCR4 antagonist, attenuates allergic lung inflammation and airway hyperreactivity. Am. J. Pathol. 2002: 160:1353-1360

Luo S, Kleemann GA, Ashraf JM, Shaw WM, Murphy CT. TGF- $\beta$ and insulin signaling regulate reproductive aging via oocyte and germline quality maintenance. Cell. 2010 Oct $15 ; 143(2): 299-312$.

Lühder F, Reichardt HM. Traditional concepts and future avenues of glucocorticoid action in experimental autoimmune encephalomyelitis and multiple sclerosis therapy. Crit Rev Immunol. 2009;29(3):255-73.

Lyons SK. Advances in imaging mouse tumour models in vivo. J Pathol. 2005 Jan;205(2):194-205.

Macfarlane DP, Forbes S, Walker BR. Glucocorticoids and fatty acid metabolism in humans: fuelling fat redistribution in the metabolic syndrome. J Endocrinol. 2008 May;197(2):189204.

March ME, Sleiman PMA, Hakonarson H. The Genetics of Asthma and Allergic Disorders. Discov Med. 2011 Jan;11(56):35-45. 
Martinez FO, Helming L, Gordon S. Alternative activation of macrophages: an immunologic functional perspective. Annual Reviews. 2009; 27:451-483.

McNally JG, Müller WG, Walker D, Wolford R, Hager GL. The glucocorticoid receptor: rapid exchange with regulatory sites in living cells. Science. 2000 Feb 18;287(5456):1262-5.

Métivier R, Penot G, Hübner MR, Reid G, Brand H, Kos M, Gannon F. Estrogen receptoralpha directs ordered, cyclical, and combinatorial recruitment of cofactors on a natural target promoter. Cell. 2003 Dec 12;115(6):751-63.

Morishima Y, Kanelakis KC, Murphy PJ, Lowe ER, Jenkins GJ, Osawa Y, Sunahara RK, Pratt WB. The hsp90 cochaperone p23 is the limiting component of the multiprotein hsp90/hsp70-based chaperone system in vivo where it acts to stabilize the client protein: hsp90 complex. J Biol Chem. 2003 Dec 5;278(49):48754-63.

Mosser DM, Edwards JP. Exploring the full spectrum of macrophage activation. Nature Reviews Immunology. 2008; 8(12):958-969.

Mulla A \& Buckingham JC. Regulation of the hypothalamopituitary-adrenal axis by cytokines. Bailliere's Best Practice Research.Clinical Endocrinology and Metabolism 1999: 13 503-521.

Nakao A, Miike S, Hatano M, Okumura K, Tokuhisa T, Ra C, Iwamoto I. Blockade of transforming growth factor beta/Smad signaling in $\mathrm{T}$ cells by overexpression of Smad7 enhances antigen-induced airway inflammation and airway reactivity. J Exp Med. $2000 \mathrm{Jul}$ 17;192(2):151-8.

Noveral JP, Grunstein MM. Role and mechanism of thromboxane-induced proliferation of cultured airway smooth muscle cells. Am J Physiol 1992; 263: L555- L561.

Noveral JP, Rosemberg SM, Anbar RA, Pawlowski NA, Grunstein MM. Role of endothelin-1 and epidermal growth factor in cultured airway smooth muscle. Am J Physiol 1992; 263: L317-L324

O'Byrne PM, Pedersen S, Busse WW, Tan WC, Chen YZ, Ohlsson SV, et al. Effects of early intervention with inhaled budesonide on lung function in newly diagnosed asthma. Chest. 2006;129:1478-1485.

Petit-Frere C., Dugas B, Braquet P, and Mencia-Huerta JM. Interleukin-9 potentiates the interleukin-4-induced $\operatorname{IgE}$ and $\mathrm{IgG1}$ release from murine B lymphocytes. Immunology. 1993: 79:146-151.

Persson CC. The role of microvascular permeability in the pathogenesis of asthma. Eur $\mathbf{J}$ Respir Dis Suppl. 1986;144:190-216. 
Pidala J, Kim J, Kharfan-Dabaja MA, Nishihori T, Field T, Perkins J, Perez L, Fernandez $\mathrm{H}$, Anasetti C. Dysglycemia following glucocorticoid therapy for acute graft-versus-host disease adversely affects transplantation outcomes. Biol Blood Marrow Transplant. 2011 Feb;17(2):239-48.

Pomper MG. Translational molecular imaging for cancer. Cancer Imaging. 2005 Nov 23;5 Spec No A:S16-26.

Pratt WB, Toft DO. Regulation of signaling protein function and trafficking by the hsp90/hsp70-based chaperone machinery. Exp Biol Med (Maywood). 2003 Feb;228(2):11133.

Préfontaine, Al-Awan DL, Mogas AK, Audusseau S, Lajoie-Kadoch S, Olivenstein R, Chakir J, Halayko AJ, Lemière C, Martin JG and Hamid Q. Interleukin-33 in asthma: insights into pro-inflammatory roles of airway structural cells. Allergy, Asthma \& Clinical Immunology 2010, 6(Suppl 1):P20

Reichardt HM, Kaestner KH., Tuckermann J, Kretz O, Wessely O, Bock R, Gass P, Schmid W, Herrlich P, Angel P. DNA binding of the glucocorticoid receptor is not essential for survival. Cell 1998: 93, 531-541.

Reichardt HM, Tuckermann JP, Gottlicher M, Vujic M, Weih F, Angel P, Herrlich P, and Schutz G. Repression of inflammatory responses in the absence of DNA binding by the glucocorticoid receptor. The EMBO journal 2001: 20, 7168-7173.

Renauld J. New insights into the role of cytokines in asthma. J Clin Pathol. 2001 August; 54(8): 577-589.

Ricci M, Matucci A, Rossi O. T cells, cytokines, IgE and allergic airways inflammation. J Investig Allergol Clin Immunol. 1994: 4(5):214-20.

Richard EM, Helbling JC, Tridon C, Desmedt A, Minni AM, Cador M, Pourtau L, Konsman JP, Mormède P, Moisan MP. Plasma transcortin influences endocrine and behavioral stress responses in mice. Endocrinology. 2010 Feb;151(2):649-59.

Rickert RC, Roes J, Rajewsky K. B lymphocyte-specific, Cre-mediated mutagenesis in mice. Nucleic Acids Res 1997: 25:1317-1318

Riffo-Vasquez Y, Spina D. Role of cytokines and chemokines in bronchial hyperresponsiveness and airway inflammation. Pharmacol. Ther. 2002: 94, 185-211.

Saalbach A, Klein C, Schirmer C, Briest W, Anderegg U, Simon JC. Dermal fibroblasts promote the migration of dendritic cells. J Invest Dermatol. 2010; 130(2):444-454

Robinson TA, Kolbb B. Structural plasticity associatedwith exposure to drugs of abuse. Neuropharmacology. 2004;47 Suppl 1:33-46. 
Roche WR, Beasley R, Williams JH, Holgate ST. Subepithelial fibrosis in the bronchi of asthmatics. Lancet 1989; i: 520-524.

Rock JR, Barkauskas CE, Cronce MJ, Xue Y, Harris JR, Liang J, Noble PW, Hogan BL. Multiple stromal populations contribute to pulmonary fibrosis without evidence for epithelial to mesenchymaltransition. Proc Natl Acad Sci U S A. 2011 Dec 27;108(52):E1475-83.

Saetta M.and Turato G. Airway pathology in asthma. Eur Respir J 2001; 18: Suppl. 34: 18s$23 \mathrm{~s}$

Saetta M, Di Stefano A, Rosina C, Thiene G, Fabbri LM. Quantitative structural analysis of peripheral airways and arteries in sudden fatal asthma. Am Rev Respir Dis 1991; 143: 138143.

Scheff JD, Calvano SE, Lowry SF, Androulakis IP. Transcriptional implications of ultradian glucocorticoid secretion in homeostasis and in the acute stress response. Physiol Genomics. 2012 Feb 1;44(2):121-9.

Schirmer C, Klein C, von Bergen M, Simon JC, Saalbach A. Human fibroblasts support the expansion of IL-17 producing T-cells via up-regulation of IL-23 production by dendritic cells. Blood. 2010;116(10):1715-1725.

Schweingruber N, Reichardt SD, Lühder F, Reichardt HM. Mechanisms of glucocorticoids in the control of neuroinflammation. J Neuroendocrinol. 2012 Jan;24(1):174-82.

Schuh J.M., Blease K., Brühl H., Mack M. and Hogaboam C.M., Intrapulmonary targeting of RANTES/CCL5-responsive cells prevents chronic fungal asthma. Eur. J. Immunol. 2003. 33: 3080-3090

Shimura S, Andoh Y, Haraguchi M, Shirato K. Continuity of airway goblet cells and intraluminal mucus in the airways of patients with bronchial asthma. Eur Respir J 1996; 9: $1395-1401$.

Sokol CL, Chu NQ, Yu S, Nish SA, Laufer TM, Medzhitov R. Basophils function as antigenpresenting cells for an allergen-induced $\mathrm{T}$ helper type 2 response. Nat. Immunol. 2009; 10:713.

Spina D. Epithelium smooth muscle regulation and interactions. Am J Respir Crit Care Med. 1998 Nov;158(5 Pt 3):S141-5.

Stahn C, Löwenberg M, Hommes DW, Buttgereit F. Molecular mechanisms of glucocorticoid action and selective glucocorticoid receptor agonists. Mol Cell Endocrinol. 2007;275(1-2):718 .

Stellato C. "Post-transcriptional and Nongenomic Effects of Glucocorticoids",Proceedings of the American Thoracic Society, Vol. 1, Inhaled corticosteroids in the treatment of asthma and COPD. 2004: pp. 255-263 
Stewart AG, Grigoriadis G, Harris T. Mitogenic actions of endothelin-1 and epidermal growth factor in cultured airway smooth muscle. Clin Exp Pharmacol Physiol 1994; 21: 277-285

Stout RD, Suttles J. Functional plasticity of macrophages: reversible adaptation to changing microenvironments. Journal of Leukocyte Biology. 2004;76(3):509-513.

Surjit M, Ganti KM,Mukherji A,Ye T,Hua G,Metzger D,Li M,Chambon P. Widespread Negative Response Elements Mediate Direct Repression by Agonist- Liganded Glucocorticoid Receptor. Cell. 2011 Apr 15;145(2):224-41. doi: 10.1016/j.cell.2011.03.027.

Takanashi S, Hasegawa Y, Kanehira Y, Yamamoto K, Fujimoto K, Satoh K, Okamura K. Interleukin-10 level in sputum is reduced in bronchial asthma, COPD and in smokers. Eur Respir J. 1999 Aug;14(2):309-14.

Tang C, Inman MD, van Rooijen N, et al. Th type 1-stimulating activity of lung macrophages inhibits Th2-mediated allergic airway inflammation by an IFN- $\gamma$-dependent mechanism. Journal of Immunology. 2001; 166(3):1471-1481.

Thomas, P.S., Yates, D.H., Barnes, P.J. Tumor necrosis factor- $\alpha$ increases airway responsiveness and sputum neutrophils in normal human subjects. Am. J. Respir. Crit. Care Med. 1995: 152:76-80.

Tonnel, A. B., Gosset, P. and Tillie-Leblond, I., Characteristics of the inflammatory response in bronchial lavage fluids from patients with status asthmaticus. Int. Arch. Allergy Immunol. 2001. 12

Townsend JM, Fallon GP, Matthews JD, Smith P, Jolin EH, McKenzie NA. IL-9-deficient mice establish fundamental roles for IL-9 in pulmonary mastocytosis and goblet cell hyperplasia but not T cell development. Immunity. 2000 Oct; 13(4):573-83. 4: 267-271.

Tronche F, Opherk C, Moriggl R, Kellendonk C, Reimann A, Schwake L, Reichardt HM, Stangl K, Gau D, Hoeflich A. Glucocorticoid receptor function in hepatocytes is essential to promote postnatal body growth. Genes Dev 2004: 18, 492-497.

Truyen E, Coteur L, Dilissen E, Overbergh L, Dupont LJ, Ceuppens JL, Bullens DM. Evaluation of airway inflammation by quantitative Th1/Th2 cytokine mRNA measurement in sputum of asthma patients. Thorax. 2006 Mar;61(3):202-8.

Tuckermann JP, Reichardt HM, Arribas R, Richter KH, Schütz G, Angel P. The DNA binding-independent function of the glucocorticoid receptor mediates repression of AP-1dependent genes in skin.J Cell Biol. 1999 Dec 27;147(7):1365-70.

Tuckermann JP, Kleiman A, Moriggl R, Spanbroek R, Neumann A, Illing A, Clausen BE, Stride B, Förster I, Habenicht AJ, Reichardt HM, Tronche F, Schmid W,Schütz G. Macrophages and neutrophils are the targets for immune suppression by glucocorticoids in contact allergy. J Clin Invest. 2007 May;117(5):1381-90. 
Turk, B.Emerging roles of cysteine cathepsins in disease and their potential as drug targets. Curr. Pharm. 2007: 13, 387-403.

Ugoccioni M, Mackay CR, Ochensberger B, et al. High expression of the chemokine receptor CCR3 in human blood basophils. Role in activation by eotaxin, MCP-4, and other chemokines. J Clin Invest.1997;100:1137-43.

Umesono K, Evans RM. Determinants of target gene specificity for steroid/thyroid hormone receptors. Cell. 1989 Jun 30;57(7):1139-46.

van Rijt LS, Jung S, Kleinjan A, Vos N, Willart M, Duez C, Hoogsteden HC, and Lambrecht $\mathrm{BN}$. In vivo depletion of lung CD11c+ dendritic cells during allergen challenge abrogates the characteristic features of asthma. J. Exp. Med. 2005: 201:981-991

Vasiljeva, O., Reinheckel, T., Peters, C., Turk, D., Turk, V., Turk, B. (2007): Emerging roles of cysteine cathepsins indisease and their potential as drug targets. Curr. Pharm. Des., 13, 387-403.

Vermaelen KY, Muino IC, Lambrecht BN, and Pauwels RA. Specific Migratory Dendritic Cells Rapidly Transport Antigen from the Airways to the Thoracic Lymph Nodes. J Exp Med. 2001 January 1; 193(1): 51-60

Vignola AM, Gagliardo R, Guerrera D, Chiappara G, Chanez P, Bousquet J, Bonsignore G. New evidence of inflammation in asthma. Thorax 2000;55 (Suppl 2):S59-S60

Vignola AM, Scichilone N, Bousquet J, Bonsignore G, Bellia V. Effect of age and asthma duration upon elastase and alphal-antitrypsin levels in adult asthmatics. Eur Respir J. 2003 Nov;22(5):795-801.

Wang YH, Angkasekwinai P, Lu N, Voo KS, Arima K, Hanabuchi S, Hippe A, Corrigan CJ, Dong C, Homey B, Yao Z, Ying S, Huston DP, Liu YJ.. IL-25 augments type 2 immune responses by enhancing the expansion and functions of TSLP-DC-activated Th2 memory cells. J. Exp. Med. 2007: 204:1837-1847.

Wenzel SE, Szefler SJ, Leung DY, Sloan SI, Rex MD, Martin RJ. Bronchoscopic evaluation of severe asthma: persistent inflammation associated with high dose glucocorticoids. Am J Respir Crit Care Med 1997;156:737-743.

Webster JI, Tonelli L \& Sternberg EM. Neuroendocrine regulation of immunity. Annual Review of Immunology 2002: 20 125-163.

Webster JI, Tonelli LH, Moayeri M, Simons SS, Jr., Leppla SH \& Sternberg EM 2. Anthrax lethal factor represses glucocorticoids and progesterone receptor activity. PNAS. 2003: 100 5706-5711.

Webster and Sternberg EM. Role of the hypothalamic-pituitary-adrenal axis, glucocorticoids and glucocorticoid receptors in toxic sequelae of exposure to bacterial and viral products. Journal of Endocrinology.2004: 181, 207-221 
Wills-Karp M. The gene encoding interleukin-13: a susceptibility locus for asthma and related traits. Respiratory Research 2000: 1:19-23

Wright JR. Immunoregulatory functions of surfactant proteins. Nat Rev Immunol. 2005 Jan; 5 (1): 58-68.

Wüst S, van den Brandt J, Tischner D, Kleiman A, Tuckermann JP, Gold R, Lühder F, Reichardt HM. Peripheral $\mathrm{T}$ cells are the therapeutic targets of glucocorticoids in experimental autoimmune encephalomyelitis. J Immunol. 2008;180:8434-8443.

Wu K, Bi Y, Sun K, Xia J, Wang Y, Wang C. Suppression of Allergic Inflammation by Allergen- DNA-modified Dendritic Cells Depends on the Induction of Foxp3+ Regulatory T Cells. Scand J Immunol 2008; 67: 140 - 51

Wüst S, Tischner D, John M, Tuckermann JP, Menzfeld C, Hanisch UK, van den Brandt J, Lühder F, Reichardt HM. Therapeutic and adverse effects of a non-steroidal glucocorticoid receptor ligand in a mouse model of multiple sclerosis. PLoS One. 2009 Dec 7;4(12):e8202.

Xiao H, Rizzo AN, Siegler J, Chen W. The Importance of Bronchial Epithelial Junction Integrity in Asthma. J Aller Ther. 2013: S11: 003.

Xu X, Rock JR, Lu Y, Futtner C, Schwab B, Guinney J, Hogan BL, Onaitis MW. Evidence for type II cells as cells of origin of K-Ras-induced distal lung adenocarcinoma. Proc Natl Acad Sci U S A. 2012 Mar 27;109(13):4910-5.

Xystrakis E, Kusumakar S, Boswell S, Peek E, Urry Z, Richards DF, Adikibi T, Pridgeon C, Dallman M, Loke TK, Robinson DS, Barrat FJ, O'Garra A, Lavender P,Lee TH, Corrigan C, Hawrylowicz CM. Reversing the defective induction of IL-10-secreting regulatory $\mathrm{T}$ cells in glucocorticoid-resistant asthma patients. J Clin Invest. 2006 Jan;116(1):146-55.

Yang M, Kumar RK, Hansbro PM, Foster PS. Emerging roles of pulmonary macrophages in driving the development of severe asthma. J Leukoc Biol. 2012 Apr;91(4):557-69.

Yoo J, Omori M, Gyarmati D, Zhou B, Aye T, Brewer A, Comeau MR, Campbell DJ, Ziegler SF. Spontaneous atopic dermatitis in mice expressing an inducible thymic stromal lymphopoietin transgene specifically in the skin. J Exp Med. 2005 Aug 15;202(4):541-9.

Zavasnik-Bergant T, Turk B. Cysteine cathepsins in the immune response. Tissue Antigens, 2006: 67, 349-355.

Zieg, G, Lack, G, Harbeck, RJ, Gelfand, EW, Leung, DY. In vivo effects of glucocorticoids on IgE production. J Allergy Clin Immunol 1994: 94:222-230.

Zosky GR and Sly PD. Animal models of asthma. Clin. Exp. Allergy 2007: 37, 973-988

Zhang M, Angata T, Cho JY, Miller M, Broide DH, and Varki A. Defining the in vivo function of Siglec-F, a CD33-related Siglec expressed on mouse eosinophils. Blood 2007: 109 4280-4287. 


\section{Appendix}

\subsection{List of figures}

Figure 1: Anatomy of asthma attack

Figure 2: Protective and risk factors for asthma development identified with the help of genome wide association, linkage and candidate gene studies.

Figure 3: Cells and mediators involved in the induction of allergic asthma.....

Figure 4: The interaction between the immune system and structural cells of the lungs during an inflammatory response 13

Figure 5: The mechanisms of host defense in airways and alveolus of the lungs 14

Figure 6: Effects of GCs on the HPA axis and structure of the GR. 18

Figure 7: Mechanisms of GC action.

Figure 8: Effects of trans-activation and trans-repression on the expression of antiinflammatory and pro-inflammatory mediators

Figure 9: Therapeutic effects and side effects of GC-treatment in $\mathrm{GR}^{\mathrm{wt}}$ and $\mathrm{GR}^{\mathrm{dim}}$ mice..... 24

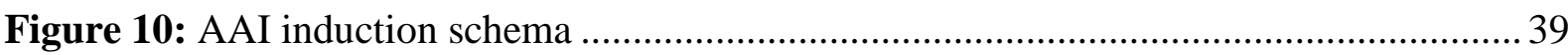

Figure 11: Reconstitution schema of bone marrow chimeras ............................................. 40

Figure 12: Histological analysis of the lung structure by HE of paraffin sections from healthy

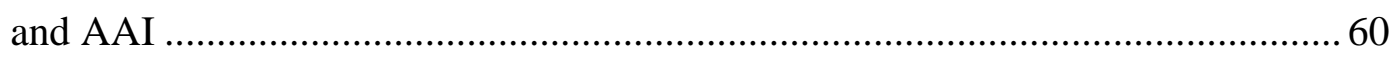

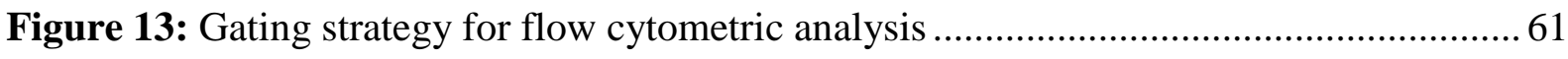

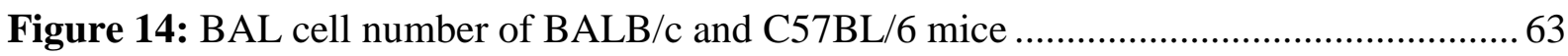

Figure 15: Analysis of the percentages of different BAL subpopulations in healthy, AAI and Dex-treated AAI wild type (BALB/c) mice

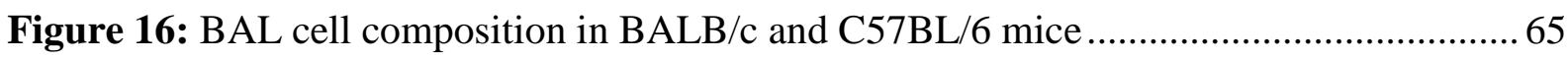

Figure 17: Antigen-specific Ig production in BALB/c and C57BL/6 wild type mice ............ 66

Figure 18: NIR in vivo imaging with ProSense680 as an imaging agent ............................ 67

Figure 19: Quantification of total fluorescence counts of activated ProSense680................. 68

Figure 20: anti-SiglecF staining of lung sections from healthy and AAI wt BALB/c mice ... 69

Figure 21: NIRF imaging with anti-SiglecF-AlexaFluor750 in vivo and ex vivo .................. 70

Figure 22: GR-expression in eosinophils from $\mathrm{GR}^{\text {flox }}$ and $\mathrm{GR}^{\mathrm{lysM}}$ mice on a BALB/c and C57BL/6 background.

Figure 23: BAL cell numbers and BAL cell composition in $\mathrm{GR}^{\mathrm{lys} M}$ mice on a BALB/c

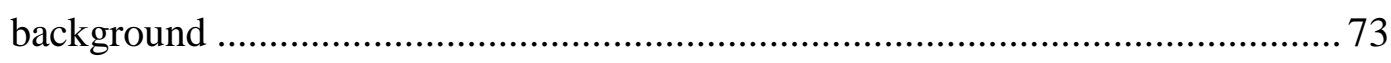

Figure 24: Proliferation and IL-2 secretion of splenocytes from $\mathrm{GR}^{\mathrm{lysM}}$ mice ..................... 74 
Figure 25: Ig isotype production in serum samples of $\mathrm{GR}^{\text {lysM }}$ and $\mathrm{GR}^{\text {flox }}$ mice on a $\mathrm{BALB} / \mathrm{c}$ background

Figure 26: BAL cell numbers and BAL cell composition of $\mathrm{GR}^{\text {lysM }}, \mathrm{GR}^{\text {lck }}, \mathrm{GR}^{\text {lysMlck }}$ and $\mathrm{GR}^{\text {flox }}$ mice on a C57BL/6 background

Figure 27: Proliferation rate of splenocytes from $\mathrm{GR}^{\text {lysM }}, \mathrm{GR}^{\mathrm{lck}}, \mathrm{GR}^{\text {lysMlck }}$ and $\mathrm{GR}^{\text {flox }}$ mice .. 77

Figure 28: Ig production and class switching in $\mathrm{GR}^{\text {lysM }}, \mathrm{GR}^{\mathrm{lck}}, \mathrm{GR}^{\mathrm{lcklysM}}$ mice 78

Figure 29: BAL cell numbers and BAL cell composition of $\mathrm{GR}^{\mathrm{CD} 19}$ and $\mathrm{GR}^{\text {flox }}$ mice on a C57BL/6 background.

Figure 30: Proliferation of splenocytes from $\mathrm{GR}^{\mathrm{CD} 19}$ and $\mathrm{GR}^{\text {flox }}$ mice 80

Figure 31: Ig production and class switching in $\mathrm{GR}^{\text {flox }}$ and $\mathrm{GR}^{\mathrm{CD} 19}$ mice 80

Figure 32: BAL cell numbers and BAL cell composition in $\mathrm{GR}^{\mathrm{CD} 11 \mathrm{c}}$ and $\mathrm{GR}^{\text {flox }}$ mice on a C57BL/6 background.

Figure 33: Proliferation and IL-2 secretion of splenocytes in $\mathrm{GR}^{\mathrm{CD} 11 \mathrm{c}}$ and $\mathrm{GR}^{\text {flox }}$ mice ........ 82

Figure 34: Ig isotype levels in the serum of $\mathrm{GR}^{\mathrm{CD} 11 \mathrm{c}}$ and $\mathrm{GR}^{\text {flox }}$ mice. 83

Figure 35: BAL cell numbers and BAL cell composition of cKit and wild type mice 84

Figure 36: Proliferation and priming in wild type and cKit mice .85

Figure 37: Absolute cell numbers in the $\mathrm{BAL}$ of $\mathrm{GR}^{\mathrm{wt}}$ and $\mathrm{GR}^{\mathrm{dim}}$ mice. 86

Figure 38: Analysis of the absolute cell number and the relative percentage of different subpopulations in BAL samples of $\mathrm{GR}^{\mathrm{wt}}$ and $\mathrm{GR}^{\mathrm{dim}}$ mice

Figure 39: Proliferation and cytokine release by splenocytes of $\mathrm{GR}^{\mathrm{wt}}$ and $\mathrm{GR}^{\mathrm{dim}}$ mice 88

Figure 40: Ova-specific Ig production in $\mathrm{GR}^{\mathrm{wt}}$ and $\mathrm{GR}^{\mathrm{dim}}$ mice 89

Figure 41: Histological analysis of the lung structure of $\mathrm{GR}^{\mathrm{wt}}$ and $\mathrm{GR}^{\mathrm{dim}}$ mice...... 90

Figure 42: NIR Imaging of $\mathrm{GR}^{\mathrm{wt}}$ and $\mathrm{GR}^{\mathrm{dim}}$ mice with ProSense680 91

Figure 43: Analysis of the IL-5 concentration in the serum of $\mathrm{GR}^{\mathrm{wt}}$ and $\mathrm{GR}^{\mathrm{dim}}$ mice. .92

Figure 44: Microvascular permeability test of $\mathrm{GR}^{\mathrm{wt}}$ and $\mathrm{GR}^{\mathrm{dim}}$ mice.

Figure 45: RT-qPCR analysis of IL-5 and Eotaxin2 mRNA expression in lung tissue and BAL samples of $\mathrm{GR}^{\mathrm{wt}}$ and $\mathrm{GR}^{\mathrm{dim}}$ mice.

Figure 46: The RT-qPCR analysis of CXCR3 and CXCR4 mRNA expression in lung tissue and BAL samples of $\mathrm{GR}^{\mathrm{wt}}$ and $\mathrm{GR}^{\mathrm{dim}}$ mice .95

Figure 47: The RT-qPCR analysis of MCP-1 and MIP-1 $\alpha$ mRNA expression in lung tissue and BAL samples of $\mathrm{GR}^{\mathrm{wt}}$ and $\mathrm{GR}^{\mathrm{dim}}$ mice. .96

Figure 48: RT-qPCR analysis of IL-4, IL-13 and RANTES mRNA expression in lung tissue and BAL samples of $\mathrm{GR}^{\mathrm{wt}}$ and $\mathrm{GR}^{\mathrm{dim}}$ mice 
Figure 49: RT-qPCR analysis of iNOS and IL-10 mRNA expression in lung tissue and BAL samples of $\mathrm{GR}^{\mathrm{wt}}$ and $\mathrm{GR}^{\mathrm{dim}}$ mice. 98

Figure 50: Reconstitution schema of hematopoietic system: bone marrow chimera types .. 100

Figure 51: Absolute cell numbers in the BAL of $\mathrm{GR}^{\mathrm{wt}}$ and $\mathrm{GR}^{\mathrm{dim}}$ chimeras .... 101

Figure 52: Analysis of absolute cell numbers of the different subpopulations in BAL of GR ${ }^{\mathrm{wt}}$ and $\mathrm{GR}^{\mathrm{dim}}$ bone marrow chimeras ..... 102

Figure 53: Proliferation and priming of splenocytes from $\mathrm{GR}^{\mathrm{wt}}$ and $\mathrm{GR}^{\mathrm{dim}}$ chimeras. 103

Figure 54: Ig isotype production of $\mathrm{GR}^{\mathrm{wt}}$ and $\mathrm{GR}^{\mathrm{dim}}$ bone marrow chimera. 104

Figure 55: RT-qPCR analysis of $\beta$-Tryptase, IL-9, TSLP, Eotaxin2, IL-25 and IL-33 mRNA expression in lung tissue samples of $\mathrm{GR}^{\mathrm{wt}}$ and $\mathrm{GR}^{\mathrm{dim}}$ chimeras 105

Figure 56: RT-qPCR analysis of RANTES, CXCR3, IL-4, IL-5 and IL-13 mRNA expression in lung tissue samples of $\mathrm{GR}^{\mathrm{wt}}$ and $\mathrm{GR}^{\mathrm{dim}}$ chimeras

Figure 57: RT-qPCR analysis of iNOS, Occludin, MCP-1, IL-10, CXCL12 and CXCR4 mRNA expression in lung tissue samples of $\mathrm{GR}^{\mathrm{wt}}$ and $\mathrm{GR}^{\mathrm{dim}}$ chimeras

Figure 58: Analysis of GR mRNA expression in AT2 cells of GR ${ }^{\text {SPCcreERT2 mice }}$ 110

Figure 59: BAL cell composition and relative percentages of BAL subpopulations of $\mathrm{GR}^{\text {flox }}$ and $\mathrm{GR}^{\mathrm{SPC} C r e E R T 2}$ mice

Figure 60: Analysis of Ig production in serum of $\mathrm{GR}^{\text {flox }}$ and $\mathrm{GR}^{\mathrm{SPC} \text { creERT2 }}$ mice 112

Figure 61: Gene expression of inflammatory mediators IL-1 $\beta$, IL-33, IL-6, iNOS, Eotaxin2, TNF- $\alpha$ and RANTES in AT2 cells from GR ${ }^{\text {flox }}$ and $\mathrm{GR}^{\mathrm{SPC} \text { SreERT2 }}$ mice ..... 114

Figure 62: Gene expression analysis of TSLP, ZO-1, Occludin and IP-10 of isolated AT2 cells from $\mathrm{GR}^{\text {flox }}$ and $\mathrm{GR}^{\mathrm{SPC} \text { CreERT2 }}$ mice 115

Figure 63: Effects of GC therapy on gene expression of inflammatory mediators in BAL and lung samples of $\mathrm{GR}^{\mathrm{wt}}$ and $\mathrm{GR}^{\mathrm{dim}}$ mice

Figure 64: Immune system dependent GC-effects on gene expression in lungs of $\mathrm{GR}^{\mathrm{wt}}$ and $\mathrm{GR}^{\mathrm{dim}}$ chimeras reconstituted with $\mathrm{GR}^{\mathrm{wt}}$ or $\mathrm{GR}^{\mathrm{dim}}$ bone marrow

Figure 65: Lung cells dependent GC-effects on gene expression in lungs of $\mathrm{GR}^{\mathrm{wt}}$ and $\mathrm{GR}^{\mathrm{dim}}$ chimeras reconstituted with $\mathrm{GR}^{\mathrm{wt}}$ or $\mathrm{GR}^{\mathrm{dim}}$ bone marrow.....

Figure 66: Effects of GC therapy on gene expression in AT2 cells isolated from Dexamethasone treated AAI mice of wild type and $\mathrm{GR}^{\text {SPCcreERT2 }}$ genotype 


\subsection{List of abbreviations}

\begin{tabular}{|c|c|}
\hline Abbreviation & Full name \\
\hline APC & Antigen presenting cell \\
\hline BAL & Bronchoalveolar lavage \\
\hline $\mathrm{BM}$ & Bone marrow \\
\hline bp & Base pair \\
\hline BSA & Bovine serum albumin \\
\hline cAMP & $3^{\prime}, 5^{\prime}$-cyclic adenosine monophosphate \\
\hline cDNA & Complementary DNA \\
\hline ConA & Concanavalin A \\
\hline cpm & Counts per minute \\
\hline DBD & DNA-binding domain \\
\hline $\mathrm{DC}$ & Dendritic cell \\
\hline Dex & Dexamethasone \\
\hline DMEM & Dulbecco's modified eagle medium \\
\hline DNA & Deoxyribonucleic acid \\
\hline dNTP & Deoxynucleotide Triphosphate \\
\hline EDTA & Ethylenediaminetetraacetic acid \\
\hline ELISA & Enzyme-linked immunosorbent assay \\
\hline FACS & Fluorescence-activated cell sorting \\
\hline FITC & Fluorescein isothiocyanate \\
\hline FSC & Forward scatter of light \\
\hline GC & Glucocorticoid \\
\hline GM-CSF & $\begin{array}{l}\text { Granulocyte macrophage colony- } \\
\text { stimulating factor }\end{array}$ \\
\hline
\end{tabular}




\begin{tabular}{|c|c|}
\hline GR & Glucocorticoid receptor \\
\hline GRE & GC response elements \\
\hline HPA axis & Hypothalamic-pituitary-adrenal axis \\
\hline HPRT & $\begin{array}{l}\text { Hypoxanthine-guanine } \\
\text { phosphoribosyltransferase }\end{array}$ \\
\hline HRP & Horseradish peroxidase \\
\hline HS & Haematopoietic system \\
\hline i.n. & Intranasal \\
\hline i.p. & Intraperitoneal \\
\hline i.v. & Intravenous \\
\hline IVC & individually ventilated cage \\
\hline IFN & Interferon \\
\hline IL & Interleukin \\
\hline MHC & Major histocompatibility complex \\
\hline OD & Optical density \\
\hline Ova & Ovalbumin \\
\hline PAMP & Pathogen-associated molecular pattern \\
\hline PBS & Phosphate-buffered saline \\
\hline PCR & Polymerase chain reaction \\
\hline PE & Phycoerythrin \\
\hline PI3K & Phosphatidylinositide 3-kinase \\
\hline SEM & Standard error of mean \\
\hline TNF & Tumour necrosis factor \\
\hline TSLP & Thymic stromal lymphopoietin \\
\hline T reg & regulatory $\mathrm{T}$ cell \\
\hline
\end{tabular}


7. Acknowledgements

First of all, I am very grateful to my supervisor Prof. Dr. Holger Reichardt for giving me the opportunity to work on these interesting projects. His continuous support, his helpful advice in combination with the possibility to discuss new ideas and controversial results open-mindedly guided me through my $\mathrm{PhD}$ time.

I would also like to thank my thesis committee members Prof. Dr. Uwe Groß and Prof. Dr. Jan Tuckermann for their interest in my project and scientific input.

Next I wish to thank Prof. Dr. Frauke Alves, Dr. Andrea Markus, Christian Dullin, Sara Greco, Rosi Streich and other members of the Department of Hematology and Oncology for their scientific and emotional support.

From the Department of Cellular and Molecular Immunology, a special thank you goes to Amina Bassibas and Kai Michel for their excellent help and valuable support through all the years.

I also need to thank the current and past members of our lab especially my student apprentice for their contribution to this work and of course Carina, Jennifer, Xiao, Elena, Katharina and Julius for a good working atmosphere and a great time together.

Finally, I would like to express my deepest gratitude to my family and friends for surrounding me with love and for the unlimited support in all circumstances. 
